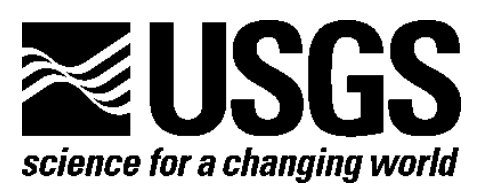

\title{
Hawaiian Volcano Observatory Seismic Data, January to December 2008
}

\author{
By Jennifer S. Nakata and Paul G. Okubo
}

Open-File Report 2009-1251

U.S. Department of the Interior

U.S. Geological Survey 


\section{U.S. Department of the Interior \\ KEN SALAZAR, Secretary}

\section{U.S. Geological Survey \\ Marcia K. McNutt, Director}

U.S. Geological Survey, Reston, Virginia: 2009

For product and ordering information:

World Wide Web: http://www.usgs.gov/pubprod

Telephone: 1-888-ASK-USGS

For more information on the USGS-the Federal source for science about the Earth, its natural and living resources, natural hazards, and the environment:

World Wide Web: $h$ ttp://www.usgs.gov

Telephone: 1-888-ASK-USGS

Suggested citation:

Nakata, J.S., and Okubo, P.G., 2009, Hawaiian Volcano Observatory seismic data, January to December 2008: U.S. Geological Survey Open-File Report 2009-1251, 84 p.

[http://pubs.usgs.gov/of/2009/1251/].

Any use of trade, product, or firm names is for descriptive purposes only and does not imply endorsement by the U.S. Government.

Although this report is in the public domain, permission must be secured from the individual copyright owners to reproduce any copyrighted material contained within this report. 


\section{Contents}

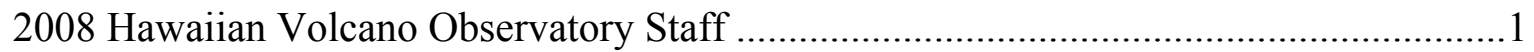

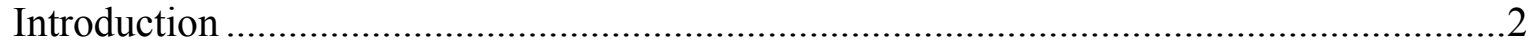

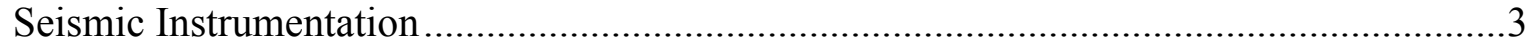

Seismic Data Processing ...................................................................................12

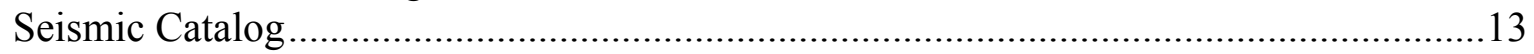

\section{Figures}

1. Map of Hawai'i Island showing geographic and geologic features .................................

2. Seismic stations operated by the USGS and NOAA on Hawai' $i$ Island ...........................

3. Seismic network telemetry scheme on Hawai'i Island .....................................................

4. A, Seismic network telemetry scheme at Kïlauea summit...............................................7

4. B, Broadband telemetry scheme at Kỉlauea summit ......................................................

5. Seismic network telemetry scheme on Maui Island........................................................

6. HVO system response curve of the four basic seismograph types ..............................11

7. Earthquake classification, shallow for Kīlauea and Mauna Loa .....................................15

8. Earthquake classification, intermediate for Kīlauea and Mauna Loa ............................16

9. Earthquake classification, crustal, for Hawai ${ }^{\dagger} i$ Island ................................................ 17

10. Earthquake classification, deep, for Hawai' $i$ Island .................................................. 18

11. Earthquake locations, Hawaiian Islands, all depths, $\mathrm{M} \geq 3.5$................................... 19

12. Earthquake locations, Hawai ‘ $i$ Island, all depths, $\mathrm{M} \geq 3.0$.......................................... 20

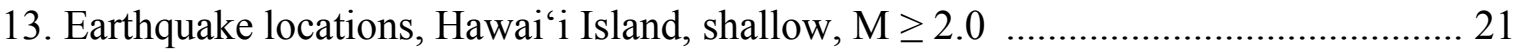

14. Earthquake locations, Hawai' $i$ Island, intermediate, $M \geq 2.0$................................. 22

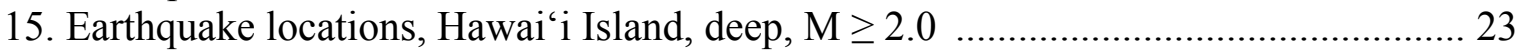

16. Earthquake locations, Kīlauea summit, shallow, $M \geq 1.0$ ………………………... 24

17. Earthquake locations, Kīlauea summit, intermediate, $M \geq 1.0$................................ 25

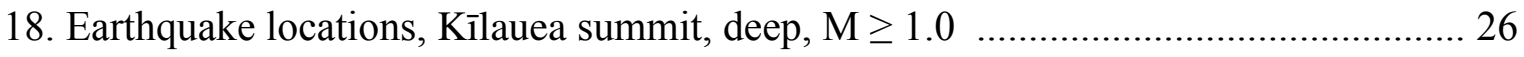

19. Earthquake locations, Kīlauea south flank, shallow, $M \geq 2.0$ …………………...... 27

20. Earthquake locations, Kîlauea south flank, intermediate, $\mathrm{M} \geq 2.0$.......................... 28

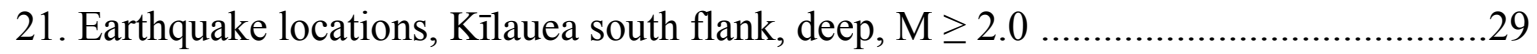

22. Earthquake locations, Mauna Loa summit, shallow, $M \geq 2.0$ ……........................ 30

23. Earthquake locations, Mauna Loa summit, intermediate, $\mathrm{M} \geq 2.0$ ……….............. 31

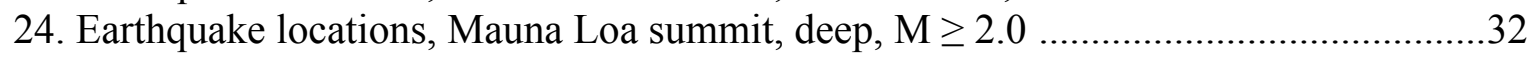

25-79. Focal solutions for events listed in Table 5 ...................................................

\section{Tables}

1. Seismic stations in Hawai' $i$ operated by the USGS ...................................................

2. Seismic instrument types in use by HVO ............................................................11

3. Coordinates of named regions used for classifying earthquakes ..................................13

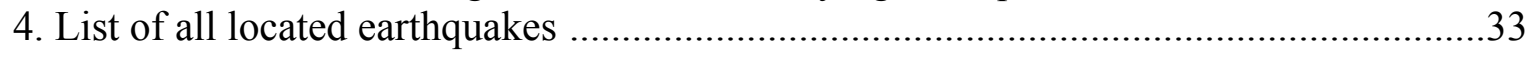

5. List of located earthquakes of magnitude 3.0 or greater .................................................69 


\title{
2008 Hawaiian Volcano Observatory Staff
}

\author{
James P. Kauahikaua (Scientist-in-Charge)
}

Steve R. Brantley (Deputy Scientist-in-Charge)

\section{Geology}

Tim R. Orr

Matthew R. Patrick

Donald A. Swanson

Frank A. Trusdell

Kelly M. Wooten

\section{Geophysics}

James P. Kauahikaua

\section{Seismology}

Steven K. Fuke (Electronics)

Bruce T. Furukawa (Electronics)

Kenneth T. Honma (Electronics)

Jennifer S. Nakata

Paul G. Okubo

Jeff O. Uribe

David C. Wilson

\section{Deformation}

Kevan Kamabayashi

Asta Miklius

Michael P. Poland

Maurice K. Sako

\section{Geochemistry}

Tamar Elias

A. Jefferson Sutton

\section{Administration}

Pauline N. Fukunaga (Webmaster)

Marian M. Kagimoto

Scientist Emeritus

C. Christina Heliker

Robert Y. Koyanagi

Arnold T. Okamura

Post-Doctoral

Takuji Yamada+-Seismology

SCEP

David Trang

Contracts

Seismology:

L. Gladys Forbes-record changing

Adolph R. Teves-record changing

CSAV Cooperative Employees

Sara E. Abraham*-Seismology

Loren Antolik-Deformation

David Whilldin*-Seismology

+Arrived in 2008.

*Left in 2008.

\section{Support}

Janet Babb (Media/Web Content/Outreach)

Robert Lopaka Lee (Computer)

T. Jane Takahashi (Library/Photo Archive) 


\section{Introduction}

The U.S. Geological Survey (USGS), Hawaiian Volcano Observatory (HVO) summary presents seismic data gathered during the year. The seismic summary is offered without interpretation as a source of preliminary data and is complete in that most data for events of $\mathrm{M} \geq 1.5$ are included. All latitude and longitude references in this report are stated in Old Hawaiian Datum.

The HVO summaries have been published in various forms since 1956. Summaries prior to 1974 were issued quarterly, but cost, convenience of preparation and distribution, and the large quantities of data necessitated an annual publication, beginning with Summary 74 for the year 1974. Beginning in 2004, summaries are simply identified by the year, rather than by summary number.

Summaries originally issued as administrative reports were republished in 2007 as Open-File Reports. All the summaries since 1956 are listed at http://geopubs.wr.usgs.gov/ (last accessed 09/21/2009).

In January 1986, HVO adopted CUSP (C California Institute of Technology USGS Seismic Processing). Summary 86 includes a description of the seismic instrumentation, calibration, and processing used in recent years. The present summary includes background information about the seismic network to provide the end user an understanding of the processing parameters and how the data were gathered.

A report by Klein and Koyanagi (1980) $)^{1}$ tabulates instrumentation, calibration, and recording history of each seismic station in the network. It is designed as a reference for users of seismograms and phase data and includes and augments the information in the station table in this summary.

Figures 11-14 are maps showing computer-located hypocenters. The maps were generated using the Generic Mapping Tools (GMT http://gmt.soest.hawaii.edu/, last accessed 09/21/2009) in place of traditional Qplot maps.

Acknowledgment:

We thank Frederique le Clerc for creating the GMT script and template files for the location maps.

${ }^{1}$ Klein, F.W., and Koyanagi, R.Y., 1980, Hawaiian Volcano Observatory seismic network history, 1950-1979: U.S. Geological Survey Open-File Report 80-302, 84 p. 


\section{Seismic Instrumentation}

\section{The Network}

The Hawaiian Volcano Observatory maintains an extensive telemetered seismic network on the Island of Hawai ' $i$. The standard HVO field sensors, 1-Hz geophones, are deployed as single-component, vertical-only units or as three-component combinations of one vertical and two orthogonal horizontal units. The 2008 network consisted of 48 station sites: 10 threecomponent, 1 six-component (which included a three-component Kinemetric Force-Balance accelerometer), 2 four-component (Uwēkahuna included a low-gain vertical with a unity gain setting; 'Ainapō included a moderate-gain vertical with a 48db setting), 2 two-component (each site included a moderate-gain vertical with a 48-db setting), and 33 vertical-component-only sites. The network coverage is most dense on and around Killauea Volcano. During 1999, HVO added to the network three verticalcomponent-only sites on the Island of Maui; these sites were not in operation during 2008. All seismic signals from the network are telemetered in real time to HVO for recording.

The National Ocean and Atmospheric Administration (NOAA), Pacific Tsunami Warning Center (PTWC), operates and maintains a network of stations on the islands of Hawai ' 1 , Maui, and O'ahu. In 1999, radio links were established to share real-time data between PTWC and HVO. PTWC signals from one $\mathrm{O}$ ‘ahu three-component station and one Maui and five Hawai 'i verticalcomponent-only stations were telemetered to HVO for recording. During 2008, telemetered signals were converted to real-time internet data streams between HVO and PTWC.

Figure 1 is a map of selected geographic and geologic features. Figure 2 shows the sites of seismic stations operated by HVO and PTWC on the Island of Hawai' $i$ during 2008. Figure 3 indicates the telemetry scheme for the seismic stations on Hawai' $i$ Island, and figures 4 shows expanded views of the telemetry schemes at Killauea summit $-4 A$, HVO seismic stations and $4 B$, a broadband network installed by the USGS Menlo Park office that is maintained by HVO. Figure 5 indicates the telemetry scheme for the seismic stations on Maui Island.

Table 1 lists seismic stations by site name, four-letter component codes, coordinates in degrees and minutes, elevation in meters, and other data, as described below, pertaining to each component. The list includes all station components operated by HVO during 2008 and reviewed in CUSP by the data analysts. All station names with field sensors installed at the site remained on the list, though operation may not have been continuous. Seismic-station components operated by PTWC on the Islands of Hawai' $i$, $\mathrm{O}^{\prime} \mathrm{ahu}$, and Maui are also listed. Phase readings from PTWC stations that are not telemetered to HVO are used to supplement data for local earthquakes and earthquakes that occur within the Hawaiian Archipelago but are distant from the Hawai' $i$ Island network.

\section{Instrumentation and Recording}

Each telemetered station's data channel has a voltage-controlled oscillator (VCO) for FM multiplex transmission to HVO by radio. These telemetering stations are all of Type 1, Earthquake Hazards Team (EHT) standard system used in USGS seismic networks (see table 2 for details). After discrimination at the receiver, signals pass through an analog-to-digital converter as part of the routine computer location processing and archiving. Through July 2001, continuous signals from the telemetered network were saved on 4-mm digital-audio tape (DAT) recording units. Three DAT recorders ran in automatic rotation as each 20 -hr tape was filled. Optic recordings are coded in table 1 as follows: H, Helicorder paper, and I, ink paper. DAT and paper records are archived at HVO.

Beginning in April 2007, continuous signals have been archived locally to a hard disk, though they were not accessible for viewing on the CUSP timing platform.

\section{Seismograph Response and Calibration}

The response curve for the short-period seismograph type in use is given in figure 6. The Type 1 curve gives the magnification of the standard EHT system from ground motion at the seismometer to the seismic trace, as would be seen on a 20x Develocorder film viewer. The curve plots the unit response, which is multiplied by CAL, a station's constant but known calibration factor, to get the response for that station. Individual CAL factors for Type 1 seismographs are Develocorder-equivalent peak-to-peak amplitudes, measured in millimeters, of a 100-microvolt 5- to 8-Hz signal introduced to the preamp/VCO in place of the geophone at the field station. The calibration process normally is performed each time a station is visited for other required maintenance. Though Develocorder operations have ceased, calculations continue to be based on Develocorder equivalents. 


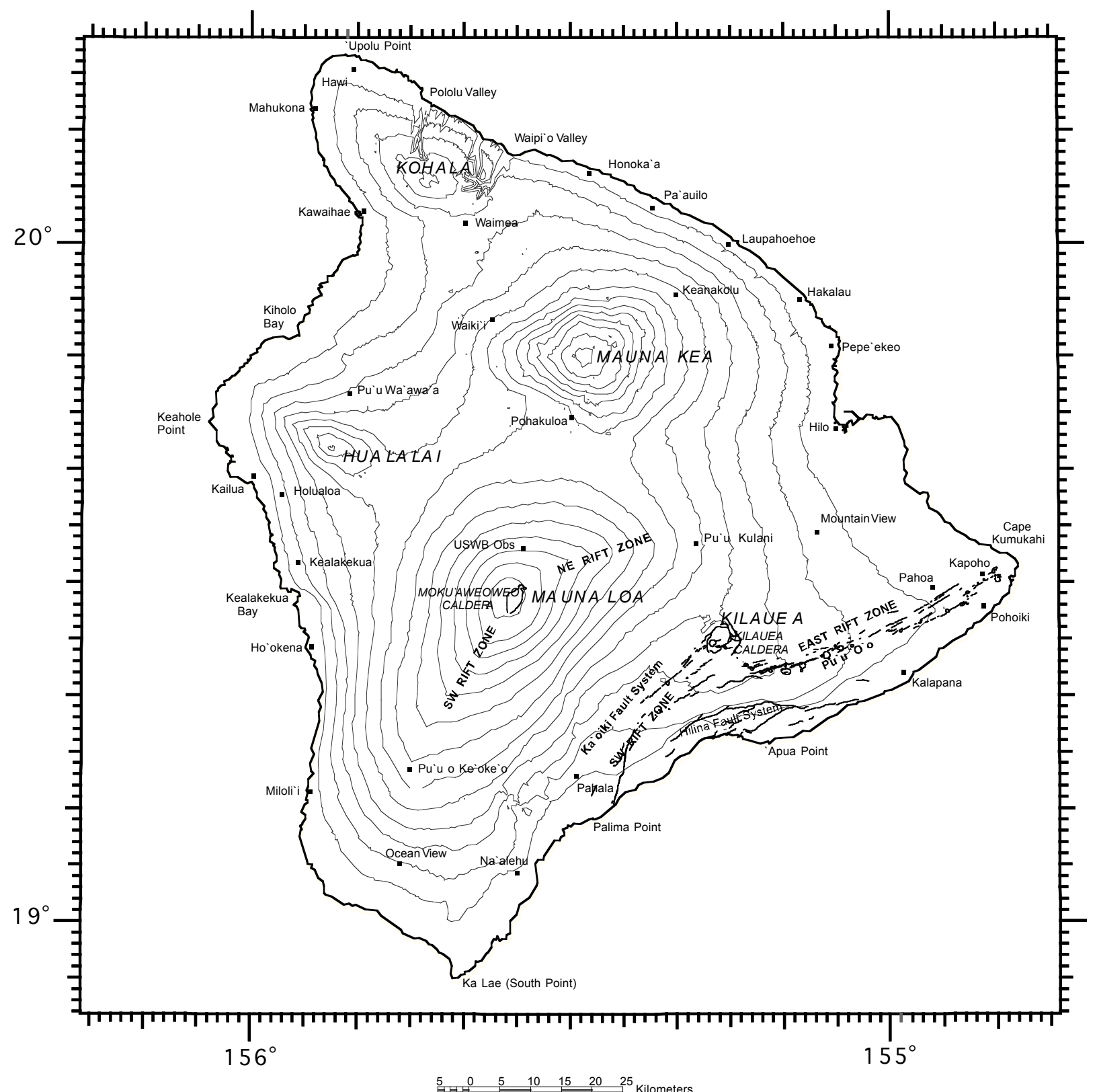

Figure 1. Map of Hawai' $i$ Island showing principal settlements and other selected geologic features. The contours are in 1,000-foot intervals. 


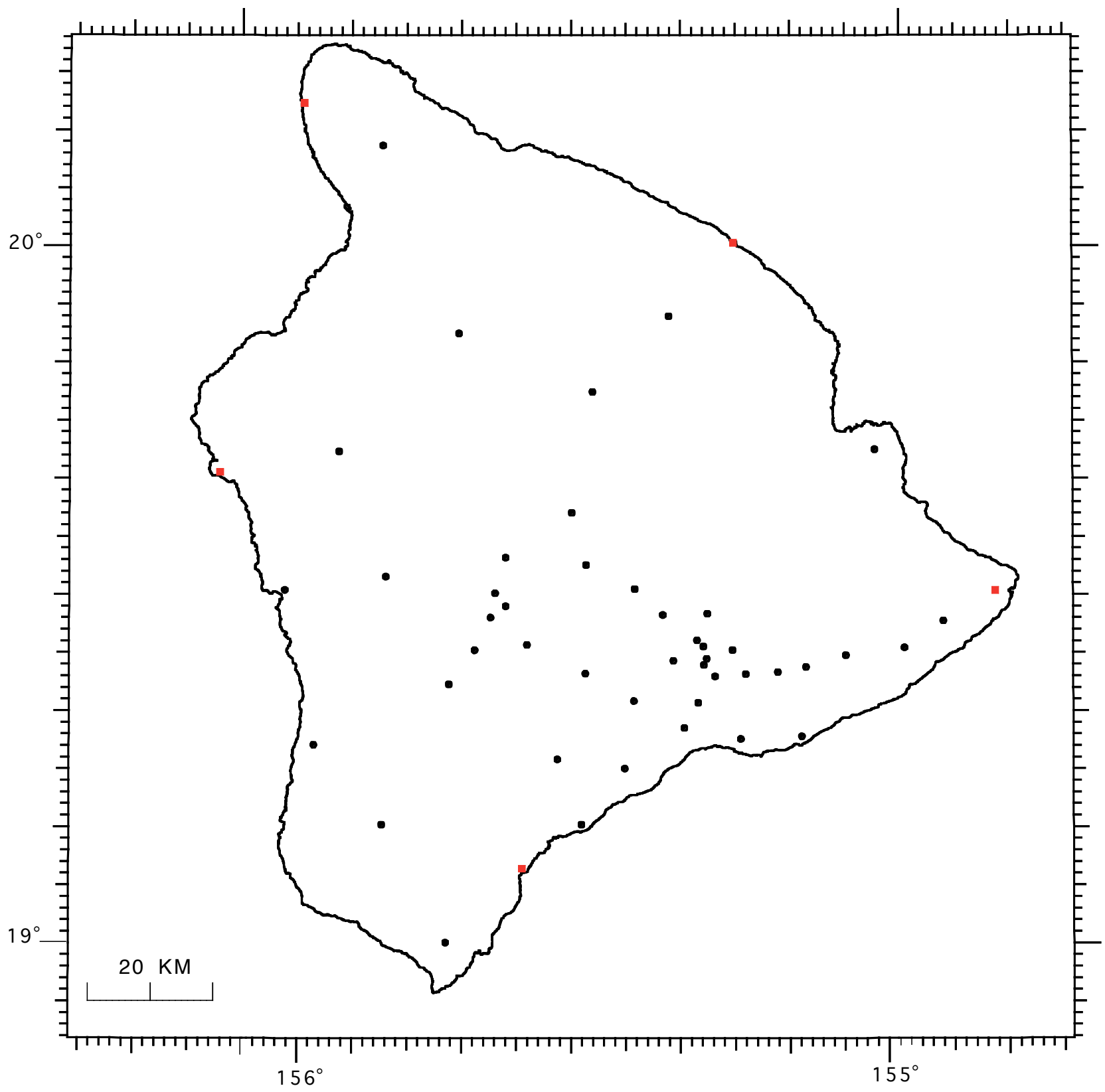

- Hawaiian Volcano Observatory network site

- Pacific Tsunami Warning Center station site

Figure 2. The 2008 Hawaiian Volcano Observatory and Pacific Tsunami Warning Center seismic network on Hawai'i Island. 


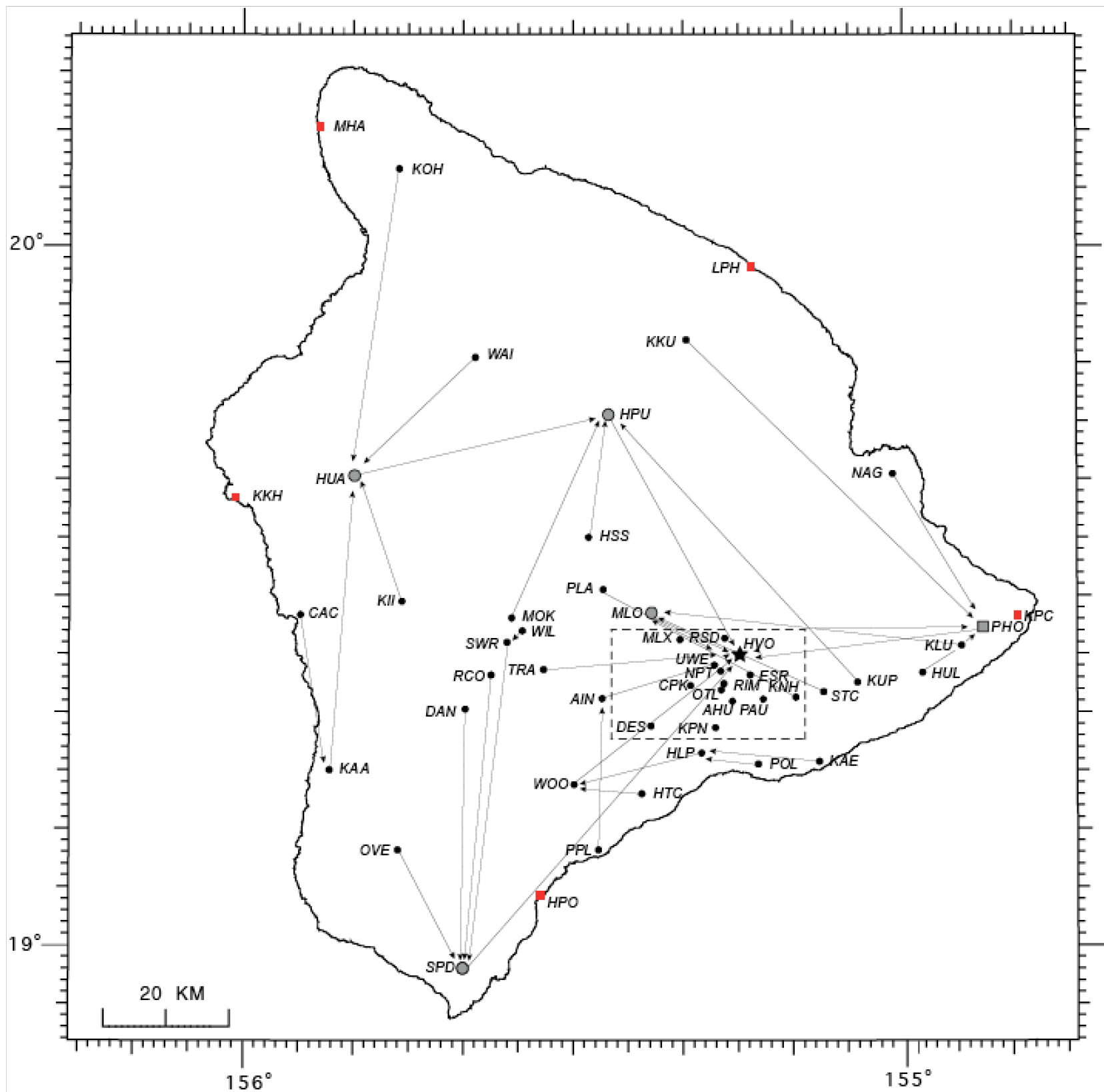

- Hawaiian Volcano Observatory (HVO)

- Hawaiian Volcano Observatory network site

- Direct-to-Line 32 Channel

- Direct-to-Line 32 Channel repeater site

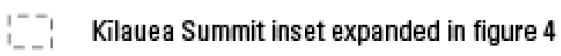

- Pacific Tsunami Warning Center station site

Figure 3. Telemetry scheme for the 2008 Hawaiian Volcano Observatory and Pacific Tsunami Warning Center seismic network on Hawai 'i Island. Figure 4 is an expanded view of Kīlauea summit, indicated by the broken line. 

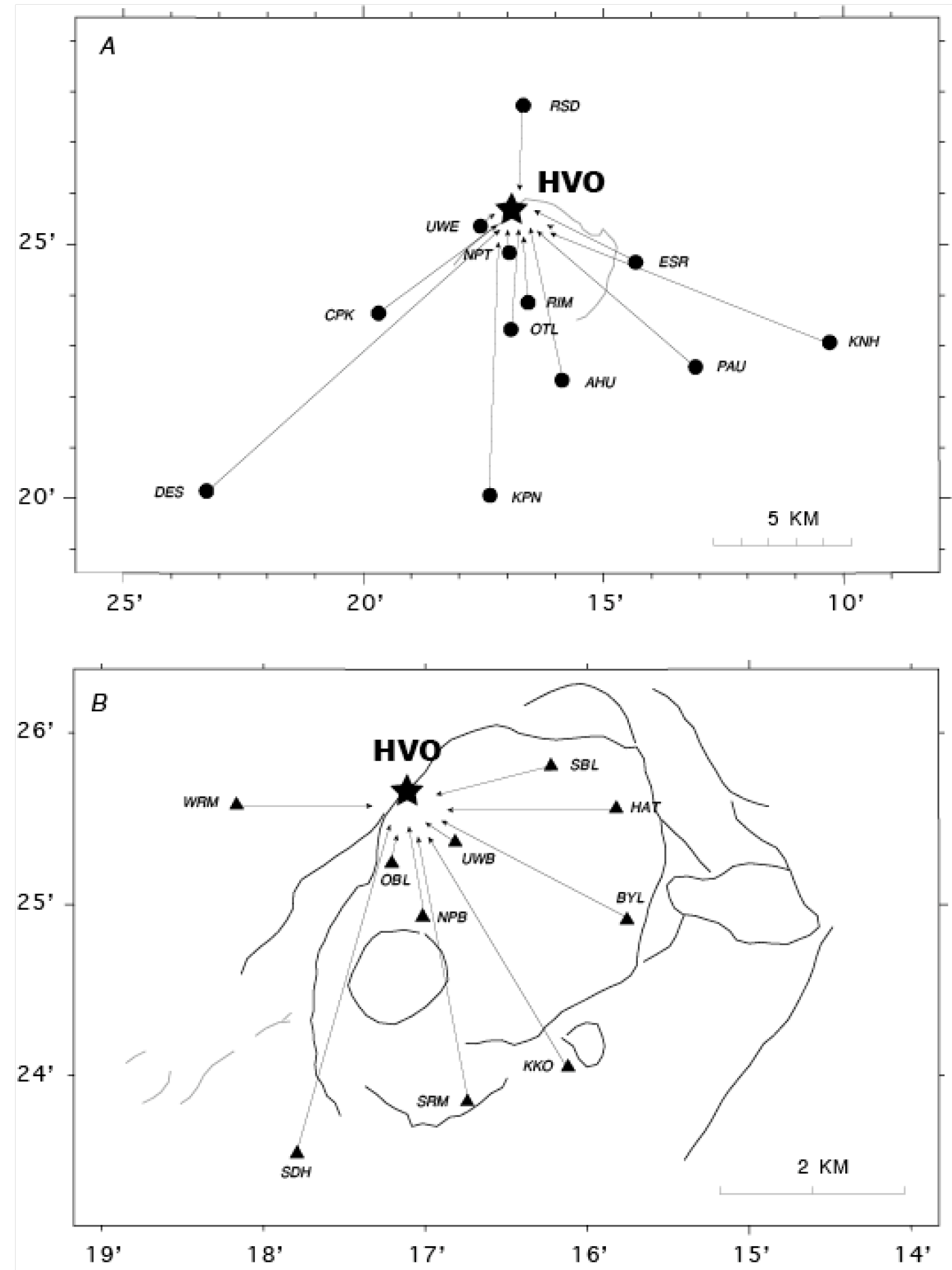

\section{$\star \quad H a w a i i a n$ Volcano Observatory (HVO) \\ - Hawailan Volcano Observatory network site \\ A USGS Menlo Park broadband site}

Figure 4. Expanded view of the Kîlauea summit inset in figure 3 showing the telemetry scheme for $A$, the 2008 Hawaiian Volcano Observatory seismic network sites and B, the 2008 U.S. Geological Survey Menlo Park broadband seismic network on Hawai'I Island. 


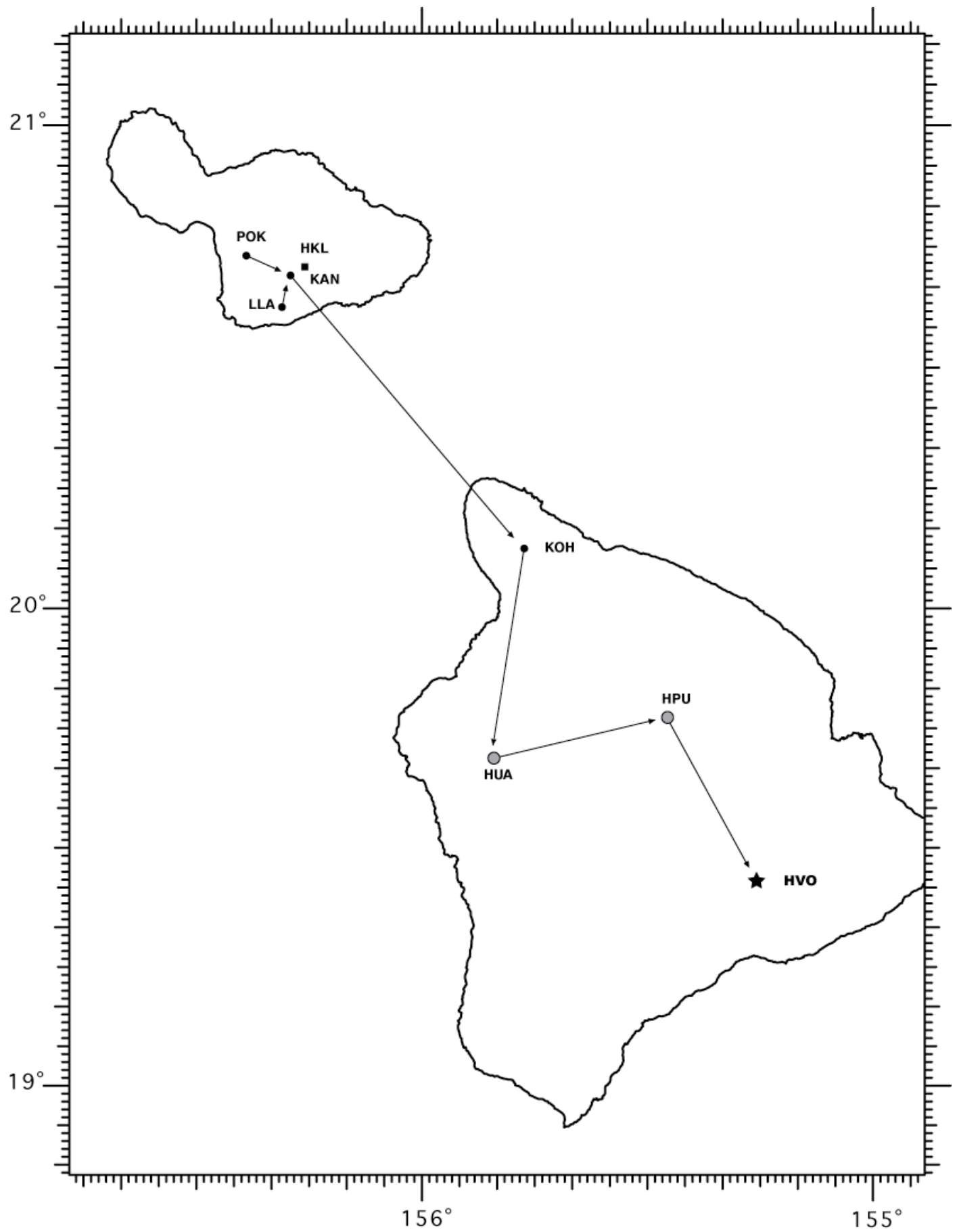

- Hawaiian Volcano Observatory (HVO)

- Hawaiian Volcano Observatory network site

- Direct-to-Line 32 Channel

- Pacific Tsunami Warning Center station site

Figure 5. Telemetry scheme for the 2008 Hawaiian Volcano Observatory and Pacific Tsunami Warning Center seismic network on Maui Island, Hawai' $i$. The HVO stations were not in operation and, thus, produced no phase data for the 2008 catalog. 
Table 1. Seismic-station sites and components operated by the U.S. Geological Survey in Hawai'i during 2008.

[During the year, there may have been outage periods that required station maintenance at certain sites.]

\begin{tabular}{|c|c|c|c|c|c|c|c|c|c|c|c|c|}
\hline \multirow{2}{*}{ STATION NAME } & \multirow[t]{2}{*}{ CODE } & \multicolumn{2}{|c|}{--LAT--- } & \multicolumn{2}{|c|}{$---\mathrm{LON}---$} & \multirow{2}{*}{$\begin{array}{r}\text { ELEV } \\
(M)\end{array}$} & \multirow{2}{*}{$\begin{array}{c}\text { DELAY } \\
1\end{array}$} & \multirow{2}{*}{$\begin{array}{c}\text { DELAY } \\
2\end{array}$} & \multirow{2}{*}{ CAL } & \multicolumn{2}{|c|}{ SEIS } & \\
\hline & & I & M & D & M & & & & & & & \\
\hline AHUA & AHUV & 19 & 22.40 & 155 & 15.90 & 1070 & -0.10 & -0.13 & 2.6 & L5 & & I \\
\hline AHUA & AHUE & 19 & 22.40 & 155 & 15.90 & 1070 & -0.10 & -0.13 & 3.0 & E5 & MW & \\
\hline AHUA & AHUN & 19 & 22.40 & 155 & 15.90 & 1070 & -0.10 & -0.13 & 3.0 & E5 & MW & \\
\hline AINAPO & AINV & 19 & 22.50 & 155 & 27.62 & 1524 & 0.13 & 0.17 & 6.8 & L5 & & \\
\hline AINAPO & AINE & 19 & 22.50 & 155 & 27.62 & 1524 & 0.13 & 0.17 & 3.0 & L5 & MW & \\
\hline AINAPO & AINN & 19 & 22.50 & 155 & 27.62 & 1524 & 0.13 & 0.17 & 3.0 & L5 & MW & \\
\hline AINAPO & AINZ & 19 & 22.50 & 155 & 27.62 & 1524 & 0.13 & 0.17 & 0.0 & L5 & & \\
\hline CAPTAIN COOK & $\mathrm{CACV}$ & 19 & 29.29 & 155 & 55.09 & 323 & 0.00 & -0.16 & 1.1 & L5 & & \\
\hline CONE PEAK & CPKV & 19 & 23.70 & 155 & 19.70 & 1038 & -0.26 & -0.07 & 6.0 & L5 & & \\
\hline DANDELION & DANV & 19 & 21.42 & 155 & 40.04 & 3003 & -0.27 & 0.03 & 4.3 & E5 & & \\
\hline DESERT & DESV & 19 & 20.20 & 155 & 23.30 & 815 & -0.29 & -0.13 & 4.5 & L5 & & I \\
\hline DIAMOND HEAD, O & ADHHZ & 21 & 16.12 & 157 & 48.25 & 137 & 0.00 & 0.00 & 0.0 & $\mathrm{~S} 13$ & & \\
\hline ESCAPE ROAD & ESRV & 19 & 24.68 & 155 & 14.33 & 1177 & -0.17 & -0.19 & 1.2 & L5 & & \\
\hline HALEAKALA, MAUI & HKLZ & 20 & 42.63 & 156 & 15.55 & 3051 & 0.00 & 0.00 & 0.0 & $\mathrm{~S} 13$ & & \\
\hline HILINA PALI & HLPV & 19 & 17.96 & 155 & 18.63 & 707 & 0.02 & 0.07 & 2.1 & L5 & & \\
\hline HONOLULU, OAHU & HONZ & 21 & 19.30 & 158 & 0.50 & 2 & 0.00 & 0.00 & 0.0 & S13 & & \\
\hline HONOLULU, OAHU & HONE & 21 & 19.30 & 158 & 0.50 & 2 & 0.00 & 0.00 & 0.0 & S13 & & \\
\hline HONOLULU, OAHU & HONN & 21 & 19.30 & 158 & 0.50 & 2 & 0.00 & 0.00 & 0.0 & S13 & & \\
\hline HONUAPO & HPOZ & 19 & 5.34 & 155 & 33.23 & 15 & 0.00 & 0.00 & 0.0 & $\mathrm{~S} 13$ & & \\
\hline HALE POHAKU & HPUV & 19 & 46.72 & 155 & 27.54 & 3396 & 0.31 & 0.17 & 3.3 & L5 & & \\
\hline HUMUULA SHEEP S & THSSV & 19 & 36.31 & 155 & 29.13 & 2445 & 0.20 & 0.35 & 4.0 & L5 & & \\
\hline HUMUULA SHEEP S & THSSE & 19 & 36.31 & 155 & 29.13 & 2445 & 0.20 & 0.35 & 3.0 & L5 & MW & \\
\hline HUMUULA SHEEP S & THSSN & 19 & 36.31 & 155 & 29.13 & 2445 & 0.20 & 0.35 & 3.0 & L5 & MW & \\
\hline HOT CAVES & $\mathrm{HTCV}$ & 19 & 14.33 & 155 & 24.02 & 381 & -0.16 & -0.07 & 2.3 & $\mathrm{E} 4$ & & \\
\hline HUALALAI & HUAV & 19 & 41.25 & 155 & 50.32 & 2189 & 0.67 & 0.38 & 2.8 & L5 & & \\
\hline HEIHEIAHULU & HULV & 19 & 25.13 & 154 & 58.72 & 369 & -0.17 & -0.16 & 1.6 & L5 & & $\mathrm{H}$ \\
\hline HEIHEIAHULU & HULE & 19 & 25.13 & 154 & 58.72 & 369 & -0.17 & -0.16 & 3.0 & E5 & MW & \\
\hline HEIHEIAHULU & HULN & 19 & 25.13 & 154 & 58.72 & 369 & -0.17 & -0.16 & 3.0 & L5 & MW & \\
\hline KAAPUNA & KAAV & 19 & 15.98 & 155 & 52.28 & 524 & -0.12 & -0.01 & 3.3 & E5 & & \\
\hline KAENA POINT & $\mathrm{KAEV}$ & 19 & 17.35 & 155 & 7.95 & 37 & -0.01 & 0.06 & 1.4 & L5 & & \\
\hline KANAHAU, MAUI & KANV & 20 & 41.60 & 156 & 17.84 & 2745 & 0.00 & 0.00 & 0.0 & L5 & & \\
\hline KANEKII & KIIV & 19 & 30.56 & 155 & 45.90 & 1841 & 0.15 & 0.37 & 3.0 & L5 & & \\
\hline KANEKII & KIIE & 19 & 30.56 & 155 & 45.90 & 1841 & 0.15 & 0.37 & 3.0 & L5 & MW & \\
\hline KANEKII & KIIN & 19 & 30.56 & 155 & 45.90 & 1841 & 0.15 & 0.37 & 3.0 & L5 & MW & \\
\hline KIPAPA, OAHU & KIPZ & 21 & 25.40 & 158 & 0.90 & 2 & 0.00 & 0.00 & 0.0 & S13 & & \\
\hline KAILUA, KONA & $\mathrm{KKHZ}$ & 19 & 39.40 & 156 & 1.12 & 1 & 0.00 & 0.00 & 0.0 & S13 & & \\
\hline KEANAKOLU & KKUV & 19 & 53.39 & 155 & 20.58 & 1863 & 0.68 & 0.24 & 3.3 & L5 & & \\
\hline PUU KALIU & KLUV & 19 & 27.48 & 154 & 55.26 & 271 & -0.17 & -0.30 & 3.4 & L5 & & \\
\hline KANE NUI O HAMO & $\mathrm{KNHV}$ & 19 & 22.95 & 155 & 10.32 & 954 & -0.17 & -0.20 & 0.0 & L5 & & I \\
\hline KANE NUI O HAMO & KNHZ & 19 & 22.95 & 155 & 10.32 & 954 & -0.17 & -0.20 & 0.0 & L5 & & \\
\hline KOHALA & $\mathrm{KOHV}$ & 20 & 7.69 & 155 & 46.77 & 1166 & -0.03 & -0.17 & 6.3 & L5 & & \\
\hline KOHALA & KOHE & 20 & 7.69 & 155 & 46.77 & 1166 & -0.03 & -0.17 & 3.0 & L5 & MW & \\
\hline KOHALA & $\mathrm{KOHN}$ & 20 & 7.69 & 155 & 46.77 & 1166 & -0.03 & -0.17 & 3.0 & L5 & MW & \\
\hline KAPOHO CONE & $\mathrm{KPCZ}$ & 19 & 30.02 & 154 & 50.51 & 134 & 0.00 & 0.00 & 0.0 & $\mathrm{~S} 13$ & & \\
\hline KIPUKA NENE & KPNV & 19 & 20.10 & 155 & 17.40 & 924 & -0.11 & -0.08 & 3.5 & L5 & & \\
\hline KUPAINAHA & KUPV & 19 & 24.32 & 155 & 4.68 & 646 & -0.25 & -0.30 & 0.0 & L5 & & \\
\hline LUALAILUA, MAUI & LLAV & 20 & 37.62 & 156 & 18.62 & 683 & 0.00 & 0.00 & 0.0 & L5 & & \\
\hline LAUPAHOEHOE & LPHZ & 19 & 59.82 & 155 & 14.58 & 1 & 0.00 & 0.00 & 0.0 & S13 & & \\
\hline MAHUKONA & MHAZ & 20 & 11.27 & 155 & 54.18 & 1 & 0.00 & 0.00 & 0.0 & S13 & & \\
\hline MAUNA LOA & MLOV & 19 & 29.80 & 155 & 23.30 & 2010 & 0.03 & 0.08 & 5.6 & L5 & & I \\
\hline MAUNA LOA & MLOE & 19 & 29.80 & 155 & 23.30 & 2010 & 0.03 & 0.08 & 3.0 & L5 & MW & \\
\hline MAUNA LOA & MLON & 19 & 29.80 & 155 & 23.30 & 2010 & 0.03 & 0.08 & 3.0 & L5 & MW & \\
\hline MOKUAWEOWEO & MOKV & 19 & 29.28 & 155 & 35.98 & 4104 & 0.15 & 0.16 & 4.2 & L5 & & IH \\
\hline
\end{tabular}




\begin{tabular}{|c|c|c|c|c|c|c|c|c|c|c|c|c|}
\hline \multirow[t]{2}{*}{ STATION NAME } & \multirow[t]{2}{*}{ CODE } & \multicolumn{2}{|c|}{--LAT--- } & \multicolumn{2}{|c|}{$---\mathrm{LON}---$} & \multirow{2}{*}{$\begin{array}{c}\text { ELEV } \\
(\mathrm{M})\end{array}$} & \multirow[t]{2}{*}{$\begin{array}{c}\text { DELAY } \\
1\end{array}$} & \multirow{2}{*}{$\begin{array}{c}\text { DELAY } \\
2\end{array}$} & \multirow{2}{*}{ CAL } & \multicolumn{3}{|c|}{ SEIS OPTIC } \\
\hline & & D & $\quad M$ & D & M & & & & & & & \\
\hline NATIONAL GUARD & NAGV & 19 & 42.12 & 155 & 1.72 & 18 & 0.54 & 0.30 & 4.0 & R5 & & \\
\hline NATIONAL GUARD & NAGE & 19 & 42.12 & 155 & 1.72 & 18 & 0.54 & 0.30 & 3.0 & R5 $\mathrm{I}$ & MW & \\
\hline NATIONAL GUARD & NAGN & 19 & 42.12 & 155 & 1.72 & 18 & 0.54 & 0.30 & 3.0 & R5 1 & MW & \\
\hline NORTH PIT & NPTV & 19 & 24.90 & 155 & 17.00 & 1115 & -0.30 & -0.18 & 3.0 & L5 & & IH \\
\hline NORTH PIT & NPTE & 19 & 24.90 & 155 & 17.00 & 1115 & -0.30 & -0.18 & 3.0 & L5 1 & MW & \\
\hline NORTH PIT & NPTN & 19 & 24.90 & 155 & 17.00 & 1115 & -0.30 & -0.18 & 3.0 & L5 & MW & \\
\hline OPANA, OAHU & OPAZ & 21 & 41.45 & 158 & 0.70 & 100 & 0.00 & 0.00 & 0.0 & $\mathrm{~S} 13$ & & \\
\hline OUTLET & OTLV & 19 & 23.38 & 155 & 16.94 & 1038 & -0.19 & -0.18 & 2.6 & L5 & & \\
\hline OUTLET & OTLZ & 19 & 23.38 & 155 & 16.94 & 1038 & -0.19 & -0.18 & 0.0 & L5 & & \\
\hline OCEANVIEW ESTATH & EOVEV & 19 & 9.21 & 155 & 45.92 & 1378 & 0.00 & 0.00 & 0.0 & L5 & & \\
\hline PAUAHI & PAUV & 19 & 22.62 & 155 & 13.10 & 994 & -0.21 & -0.24 & 2.9 & L5 & & \\
\hline PAUAHI & PAUE & 19 & 22.62 & 155 & 13.10 & 994 & -0.21 & -0.24 & 3.0 & L5 1 & MW & \\
\hline PAUAHI & PAUN & 19 & 22.62 & 155 & 13.10 & 994 & -0.21 & -0.24 & 3.0 & L5 1 & MW & \\
\hline PUU ULAULA & PLAV & 19 & 32.00 & 155 & 27.67 & 2992 & -0.03 & 0.13 & 6.3 & L5 & & I \\
\hline PUUOKALI, MAUI & POKV & 20 & 44.00 & 156 & 23.32 & 511 & 0.00 & 0.00 & 0.0 & L5 & & \\
\hline POLIOKEAWE PALI & POLV & 19 & 17.02 & 155 & 13.47 & 169 & -0.02 & 0.03 & 3.4 & E5 & & \\
\hline PUU PILI & PPLV & 19 & 9.50 & 155 & 27.87 & 35 & -0.15 & -0.15 & 1.4 & E5 & & \\
\hline RED CONE & $\mathrm{RCOV}$ & 19 & 24.36 & 155 & 37.79 & 3601 & 0.00 & 0.00 & 0.0 & L5 & & \\
\hline RIM & RIMV & 19 & 23.90 & 155 & 16.60 & 1128 & -0.21 & -0.13 & 0.0 & L5 & & $\mathrm{H}$ \\
\hline RAINSHED & RSDV & 19 & 27.78 & 155 & 16.68 & 1270 & 0.06 & 0.15 & 0.0 & L5 & & \\
\hline SOUTH POINT & SPDV & 18 & 58.94 & 155 & 40.24 & 250 & -0.17 & -0.22 & 0.0 & L5 & & \\
\hline SOUTH POINT & SPDE & 18 & 58.94 & 155 & 40.24 & 250 & -0.17 & -0.22 & 0.0 & L5 1 & MW & \\
\hline SOUTH POINT & SPDN & 18 & 58.94 & 155 & 40.24 & 250 & -0.17 & -0.22 & 0.0 & L5 1 & MW & \\
\hline STEAM CRACKS & STCV & 19 & 23.30 & 155 & 7.67 & 765 & -0.25 & -0.30 & 3.4 & L5 & & $\mathrm{H}$ \\
\hline SOUTHWEST RIFT & SWRV & 19 & 27.26 & 155 & 36.30 & 4048 & 0.01 & 0.04 & 5.6 & E5 & & \\
\hline TRAIL & TRAV & 19 & 24.91 & 155 & 32.96 & 3207 & 0.00 & 0.00 & 0.0 & L5 & & \\
\hline UWEKAHUNA & URAV & 19 & 25.40 & 155 & 17.60 & 1240 & -0.21 & 0.00 & 0.0 & R5 & & \\
\hline UWEKAHUNA & URAE & 19 & 25.40 & 155 & 17.60 & 1240 & -0.21 & 0.00 & 3.0 & R5 $\mathrm{I}$ & MW & \\
\hline UWEKAHUNA & URAN & 19 & 25.40 & 155 & 17.60 & 1240 & -0.21 & 0.00 & 3.0 & R5 I & MW & \\
\hline UWEKAHUNA & UUGZ & 19 & 25.40 & 155 & 17.60 & 1240 & 0.00 & 0.00 & 0.0 & LO & & \\
\hline WAIKII & WAIV & 19 & 51.58 & 155 & 39.60 & 1433 & 0.20 & 0.35 & 0.0 & L5 & & \\
\hline WILKES CAMP & WILV & 19 & 28.15 & 155 & 35.02 & 4037 & 0.22 & 0.17 & 2.6 & E5 & & \\
\hline WILKES CAMP & WILE & 19 & 28.15 & 155 & 35.02 & 4037 & 0.22 & 0.17 & 3.0 & L5 $\mathrm{I}$ & MW & \\
\hline WILKES CAMP & WILN & 19 & 28.15 & 155 & 35.02 & 4037 & 0.22 & 0.17 & 3.0 & L5 $\mathrm{I}$ & MW & \\
\hline WAIMANALO RIDGE, & , WMRZ & 21 & 19.22 & 157 & 40.94 & 200 & 0.00 & 0.00 & 0.0 & $\mathrm{~S} 13$ & & \\
\hline WEATHER OBSERVA & TWOBV & 19 & 32.31 & 155 & 35.01 & 3396 & 0.00 & 0.00 & 0.0 & E5 & & \\
\hline WOOD VALLEY & WOOV & 19 & 15.08 & 155 & 30.12 & 909 & -0.15 & -0.06 & 2.6 & E5 & & \\
\hline
\end{tabular}


Table 2. Seismic instrument types, U.S. Geological Survey, Hawaiian Volcano Observatory.

The codes in parentheses refer to the seismometer types listed in Table 1 . Type 1 (Codes E, L, R, and 4, 5) consists of:

a) Geophone $\quad$ Electrotech EV-17 (E), Mark Products L4C (L) or Kinemetric Ranger SS1 (R); (L) and (R) are 1.0-second period moving-magnet vertical- or horizontal- (E-W and N-S) component seismometers adjusted for an output of $0.5 \mathrm{volts} / \mathrm{cm} / \mathrm{sec}$ and 0.8 , critically damped.

b) Preamp/VCO USGS/OEVE Model J502, J512 (5) voltage-controlled oscillator. Three db points for bandpass filter at 0.1 $\mathrm{Hz}$ and $30 \mathrm{~Hz}$. Signals are transmitted on audio FM carrier over cable or FM-radio link to HVO.

Code (W) Wood-Anderson torsion seismograph.

Code (MW) Horizontal-component seismograph based on a Type 1 system and modified to 3x a Wood-Anderson response.

Code (F) Kinemetric Force-Balance Accelerometer (FBA23).

Code (S13) Geotech, 1-Hz seismometer with A1 VCO operated by the Pacific Tsunami Warning Center.

PERIOD, IN SECONDS

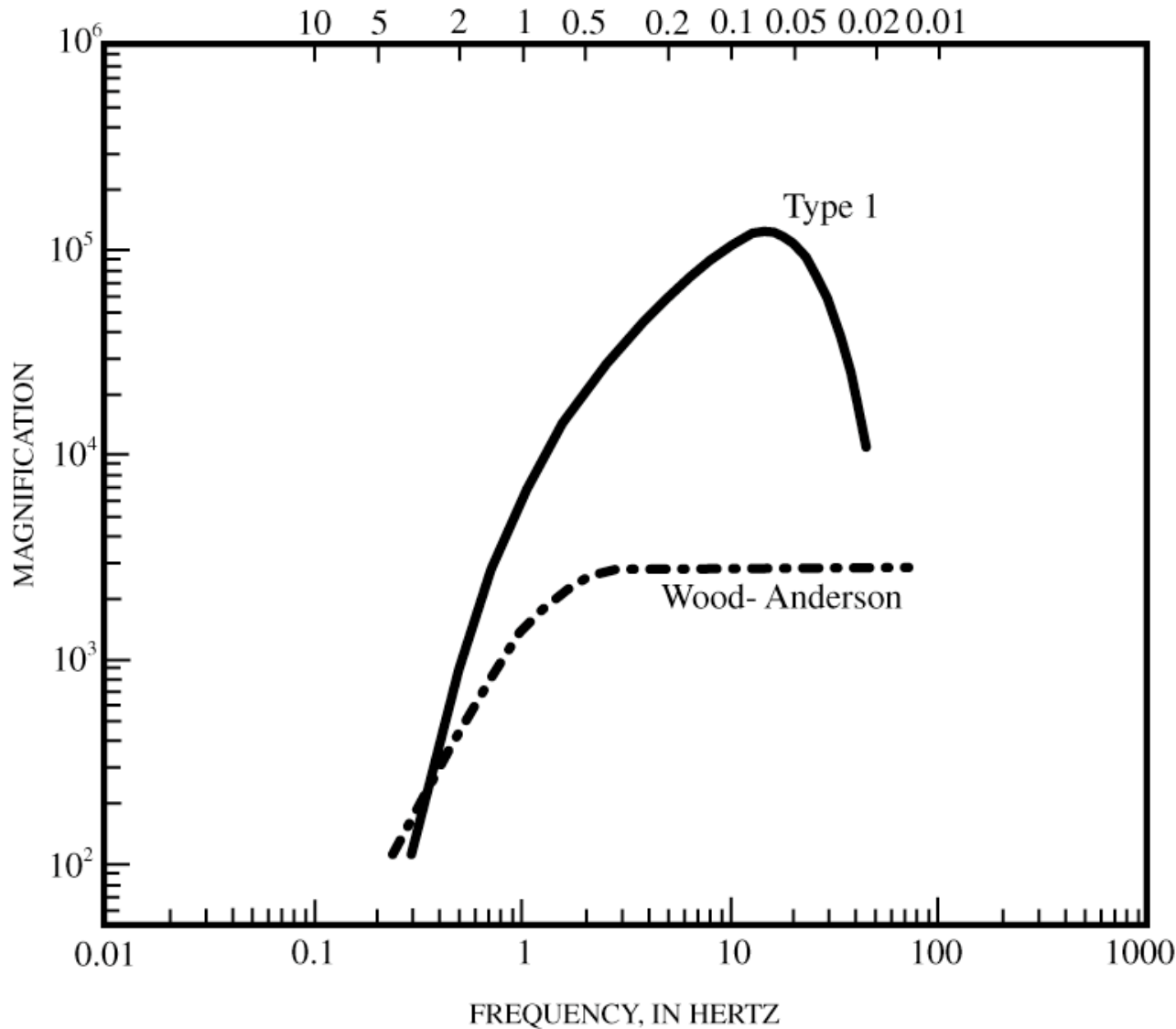

Figure 6. System-response curves for the Wood-Anderson torsion seismograph and for seismometers used by the Hawaiian Volcano Observatory. The Type 1 curve plots the unit response of the standard USGS microearthquake seismometer system as would be recorded on Develocorder film. This includes the geophone, all electronics, including telemetry, Develocorder galvanometer, and a 20x magnification film viewer. The unit response curve is multiplied by constant but known factors (CAL) to obtain the responses for individual stations. 


\section{Seismic Data Processing}

Due to age and high cost of maintenance, Develocorder 'A' was discontinued on August 1, 1997. Daily count of classified micoearthquakes from source regions around Kỉlauea and Mauna Loa, and duration of tremor, were also discontinued. Coda duration, however, is measured in seconds from drum (ink or helicorder) records to determine a coda magnitude that is entered as an external magnitude in the final solution.

In 1986, HVO acquired a VAX 11-750 computer and adopted the CUSP routine. Discriminated signals pass through an analog-to-digital converter, and detected events are saved in real time. Detected events are then demultiplexed, and P-picks are made by the computer, producing a rough location. Events are examined by an analyst on a graphics terminal in order to refine computer P-picks and to time additional P- and S-phases for a preliminary location. Binary .mem files are translated into ASCII phase files. Locations and amplitude magnitudes are then determined by using the program HYPOINVERSE-2000 (Klein, 2002$)^{2}$. Events are reworked and rerun, as needed, to produce a final solution.

Through May 2005, CUSP .grm and .mem files were archived directly onto magneto-optical media. Since June 2005, the binary files have been archived to a second Alpha node then stored on a RAID system. Files stored on the magneto-optical media through May 2005 were transferred to the RAID storage.

In July 1992, HVO acquired VAX servers and workstations needed to run the upgraded version of CUSP. The servers are used for data acquisition, and the workstations are used for interactive earthquake timing. In addition to timing $\mathrm{P}$ and $\mathrm{S}$ arrival signals, the VAX workstations were then capable of measuring peak-to-peak amplitudes, along with the associated period. This capability allowed the renewal of amplitude magnitude determinations from the network seismic stations. Amplitude data gathered from July 1992 to July 1997 became part of a test set to determine magnitude corrections for network stations. Results of newly determined magnitude corrections are detailed by Nakata and Okubo $(1997)^{3}$.

HVO currently operates Earthworm software to record all HVO seismic data, including the exchange of seismic data with cooperating networks. HVO also utilizes the Earthworm processing system for rapid computation of earthquake products (locations, magnitude, spectrograms, helicorders, ShakeMaps, and recent earthquakes Web pages). Analysis of triggered events and seismic-catalog generation is accomplished by using CUSP and HYPOINVERSE processing platforms.

Earthquake hypocenters are computed within a one-dimensional velocity model. The model is specified by velocities at four depth points, as listed below. Velocity at any depth above a homogeneous half-space is given by linear interpolation between points:

\begin{tabular}{|c|c|}
\hline $\begin{array}{l}\text { VELOCITY } \\
(\mathrm{km} / \mathrm{sec})\end{array}$ & $\begin{array}{c}\text { DEPTH } \\
(\mathrm{km})\end{array}$ \\
\hline 1.9 & 0.0 \\
\hline 6.5 & 4.6 \\
\hline 6.9 & 15.0 \\
\hline 8.3 & $\geq 16.5$ \\
\hline
\end{tabular}

Two empirical sets of station delays or corrections are used in the HYPOINVERSE locations and are given in table 1. The delay models are separated by a circle of radius $34 \mathrm{~km}$, centered at $19^{\circ} 22^{\prime} \mathrm{N}$ and $155^{\circ} 10^{\prime} \mathrm{W}$. Delay model 1 is used for epicenters occurring within a circle of radius $31 \mathrm{~km}$ from the center. This region includes Kîlauea and its south flank. A combination of both delay models is used for epicenters that fall in a transition zone that is 6-km wide. Delay model 2 is applied to the rest of the island and to offshore earthquakes. For a detailed description, refer to HYPOINVERSE-2000 (Klein, 2002) ${ }^{2}$.

Magnitudes for events are computed by using recorded amplitudes on selected network vertical, Modified Wood-Anderson (MW) horizontal, and/or moderate- and low-gain stations. Amplitude readings are corrected to an equivalent Wood-Anderson amplitude by using the curves shown in figure 6 and the CAL factors listed in table 1.

Duration magnitude is determined by the length of the signal, in seconds, read from drum recordings of Type 1 seismographs. This length of time is measured from the $\mathrm{P}$ arrival to the point where the earthquake signal has decayed to nearly the background noise level. Drum-recorded duration magnitude is calculated with a relationship equivalent to the develocorder viewer output.

\footnotetext{
${ }^{2}$ Klein, F.W., 2002, User's guide to HYPOINVERSE-2000, a Fortran Program to solve for earthquake locations and magnitudes: U.S. Geological Survey Open-File Report 02-171, 116 p.

${ }^{3}$ Nakata, J., and Okubo, P., 1997, Determination of station amplitude magnitude corrections for the Hawaiian Volcano Observatory telemetered seismograph network-Data from 1992-1997: U.S. Geological Survey Open-File Report 97-863, 73 p.
} 


\section{Seismic Catalog}

The emphasis in both station coverage and detailed data analysis is on the highly active southern half of the Island of Hawai' $\mathrm{i}$. The data set of well-recorded, located earthquakes in the Hawai' $i$ Island region is nearly complete above magnitude 2.0. Many smaller earthquakes in the Kîlauea region are locatable because of the dense instrumental coverage. Substantial effort is made to locate earthquakes elsewhere within the Hawaiian Archipelago, and although such coverage cannot be as complete as it is in southern Hawai'i, nearly all events above magnitude 4.0 are located with limited precision.

Data presented in the seismic catalog are in three parts. 1) Maps showing computer-located hypocenters are given in figures 11-24. The location maps are of different scales and provide hypocenters with magnitude thresholds set at 1.0, 2.0, 3.0, and 3.5, varying according to region. 2) The list of computer locations constitutes the bulk of this summary and is given in table 4 . Each earthquake in the list is assigned a three-letter code based on its general location and depth. Figures 7-10 are maps of the regions used to assign the location codes. The latitude and longitude limits of rectangular regions are listed in table 3 . When the listed coordinates overlap, boundary precedence is as shown in figures 7-10.3) Table 5 re-lists the events in table 4 for which the preferred magnitude is 3.0 or larger. This list includes many of the earthquakes felt in Hawai' $i$.

Table 3. Names and coordinates of regions used for classifying Hawaii earthquakes.

All earthquakes are HYPOINVERSE classified in one of the following groups, identified by a numerical class or three-letter code.

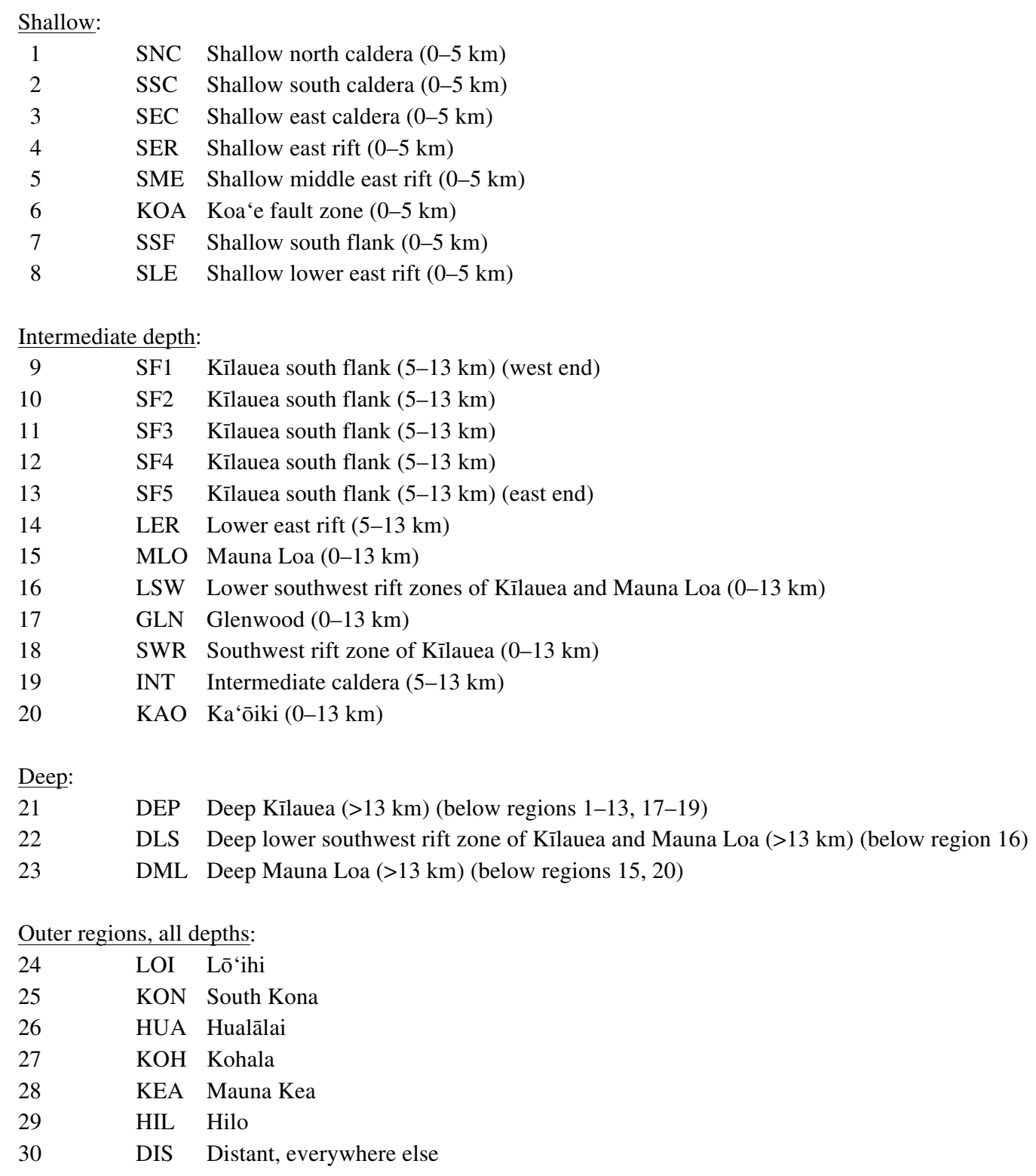


Table 3 (continued).

The latitude and longitude limits of the regions are given below. If the coordinates overlap, boundary precedence is as shown in figures $7-10$.

\begin{tabular}{|c|c|c|c|c|c|}
\hline No. & Code & N. Lat. & S. Lat. & W. Lon. & E. Lon. \\
\hline 1 & SNC & 1928.0 & 1924.5 & 15519.0 & 15514.0 \\
\hline 2 & SSC & 1924.5 & 1922.0 & 15519.0 & 15516.5 \\
\hline 3 & SEC & 1924.5 & 1922.0 & 15516.5 & 15514.0 \\
\hline 4 & SER & 1926.0 & 1920.5 & 15514.0 & 15507.2 \\
\hline 5 & SME & 1926.0 & 19 21.75-19 20.0 & 15507.2 & 15500.0 \\
\hline 6 & KOA & 1922.0 & 1920.5 & 15517.0 & 15514.0 \\
\hline 7 & SSF & $1920.6-1924.0$ & 1910.0 & 15517.0 & 15500.0 \\
\hline 8 & SLE & 1932.0 & 1916.0 & 15500.0 & 15440.0 \\
\hline 9 & SF1 & 1922.0 & 1910.0 & 15517.0 & 15514.5 \\
\hline 10 & SF2 & 1926.0 & 1910.0 & 15514.5 & 15512.3 \\
\hline 11 & SF3 & 1926.0 & 1910.0 & 15512.3 & 15509.1 \\
\hline 12 & SF4 & 1926.0 & 1910.0 & 15509.1 & 15505.3 \\
\hline 13 & SF5 & 1926.0 & 1910.0 & 15505.3 & 15500.0 \\
\hline 14 & LER & 1932.0 & 1916.0 & 15500.0 & 15440.0 \\
\hline 15 & MLO & 1935.0 & 1919.0 & 15535.0 & 15519.0 \\
\hline 16 & LSW & 1919.0 & 1840.0 & 15543.0 & 15525.0 \\
\hline 17 & GLN & 1935.0 & 1926.0 & 15519.0 & 15500.0 \\
\hline 18 & SWR & 1922.0 & 1910.0 & 15525.0 & 15517.0 \\
\hline 19 & INT & 1928.0 & 1922.0 & 15519.0 & 15514.0 \\
\hline 20 & $\mathrm{KAO}$ & 1930.0 & 1919.0 & 15532.0 & 15519.0 \\
\hline 21 & DEP & 1935.0 & 1910.0 & 15525.0 & 15500.0 \\
\hline 22 & DLS & 1919.0 & 1840.0 & 15543.0 & 15525.0 \\
\hline 23 & DML & 1935.0 & 1919.0 & 15535.0 & 15519.0 \\
\hline 24 & LOI & 1910.0 & 1840.0 & 15525.0 & 15500.0 \\
\hline 25 & $\mathrm{KON}$ & 1939.0 & 1900.0 & 15620.0 & 15543.0 \\
\hline 26 & HUA & 1955.0 & 1939.0 & 15620.0 & 15543.0 \\
\hline 27 & $\mathrm{KOH}$ & 2025.0 & 1955.0 & 15620.0 & 15534.0 \\
\hline 28 & KEA & 2025.0 & 1935.0 & 15534.0 & 15440.0 \\
\hline 29 & HIL & 1947.0 & 1932.0 & 15509.0 & 15440.0 \\
\hline
\end{tabular}




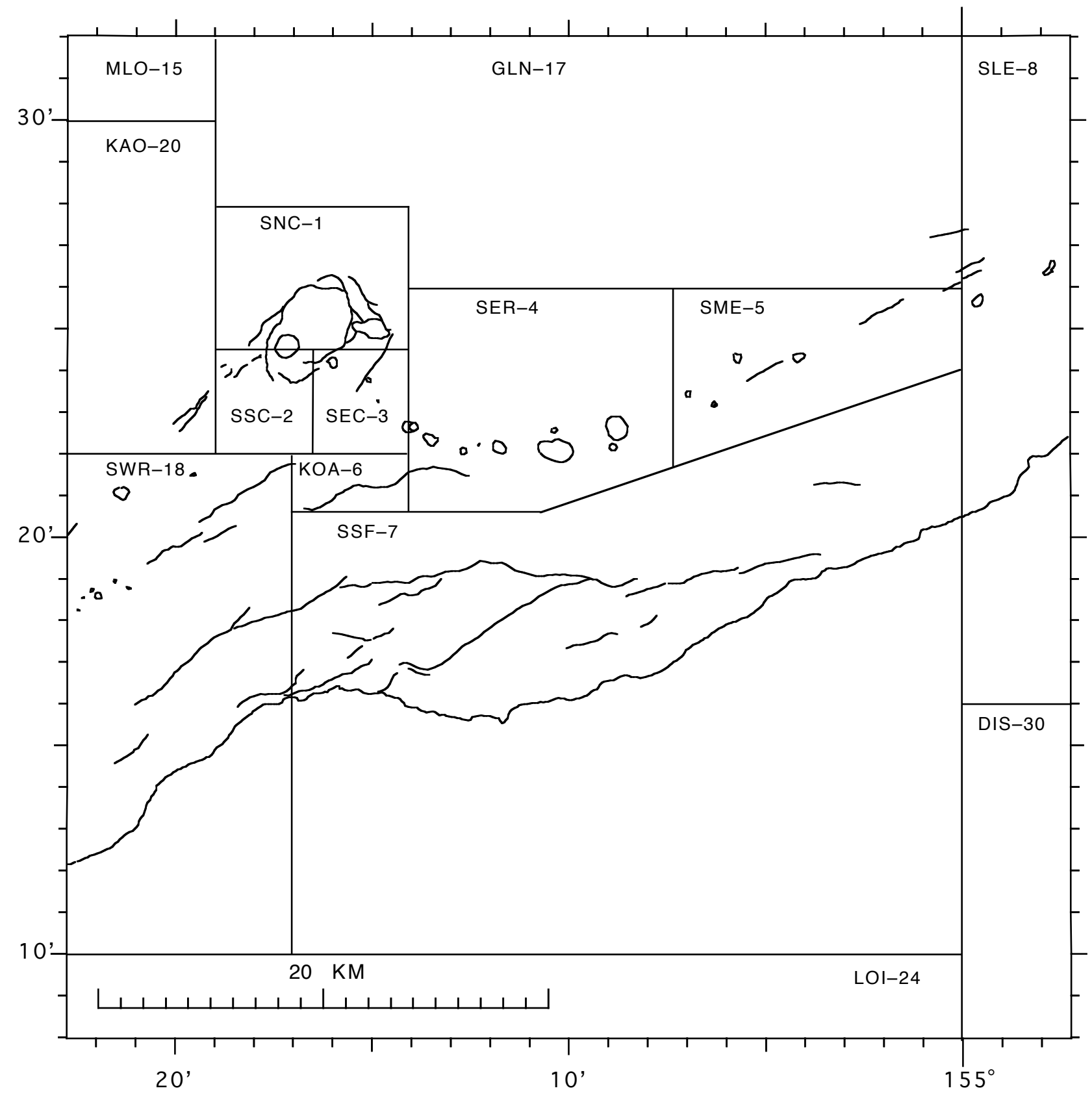

Figure 7. Regions for earthquake classification for Kîlauea and the east flank of Mauna Loa, Hawai'i. Depth range and coordinate boundaries for each region are described in table 3 . 


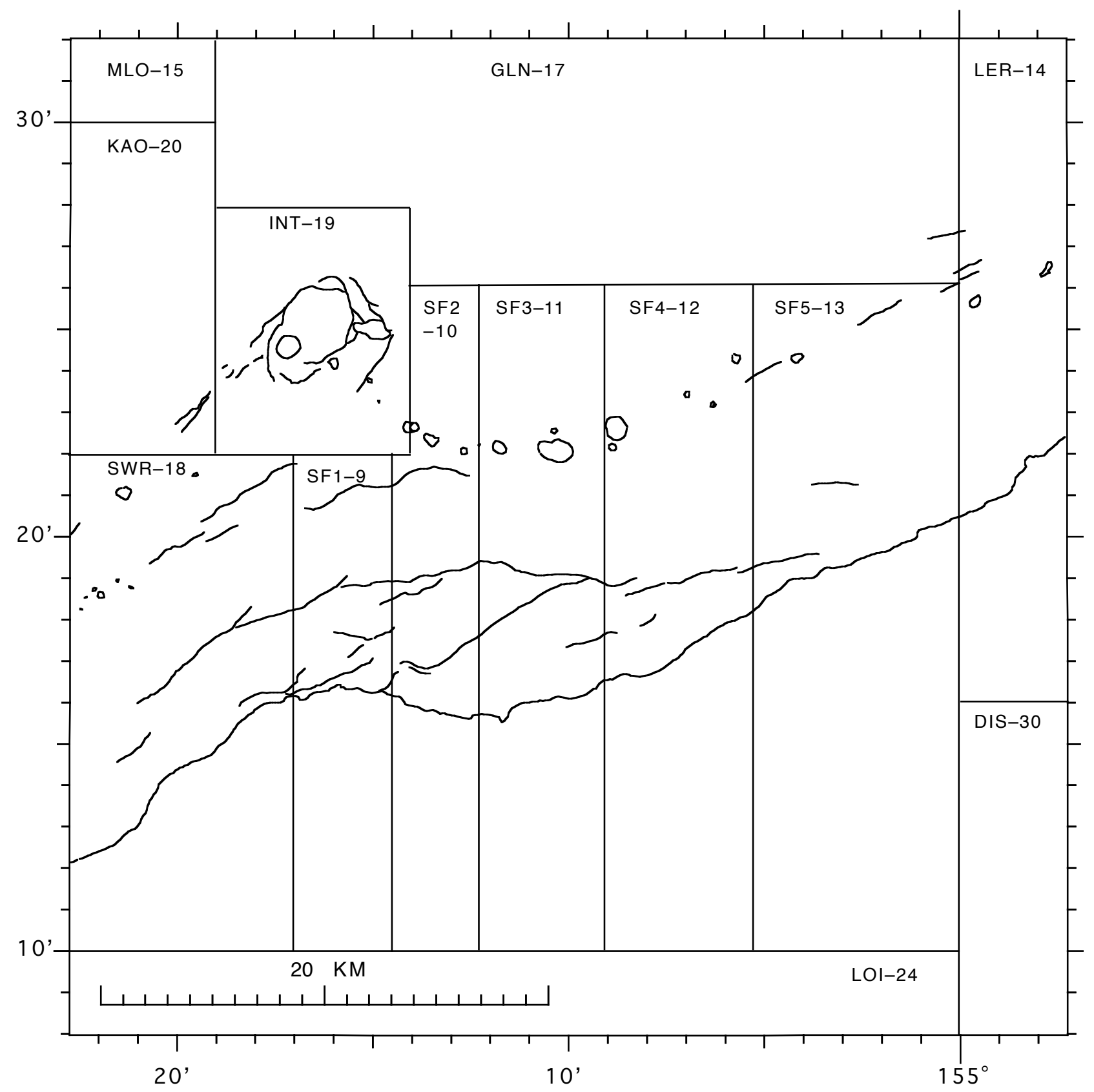

Figure 8. Regions for earthquake classification for Kîlauea and the east flank of Mauna Loa, Hawai' $i$. Depth range and coordinate boundaries for each region are described in table 3. 


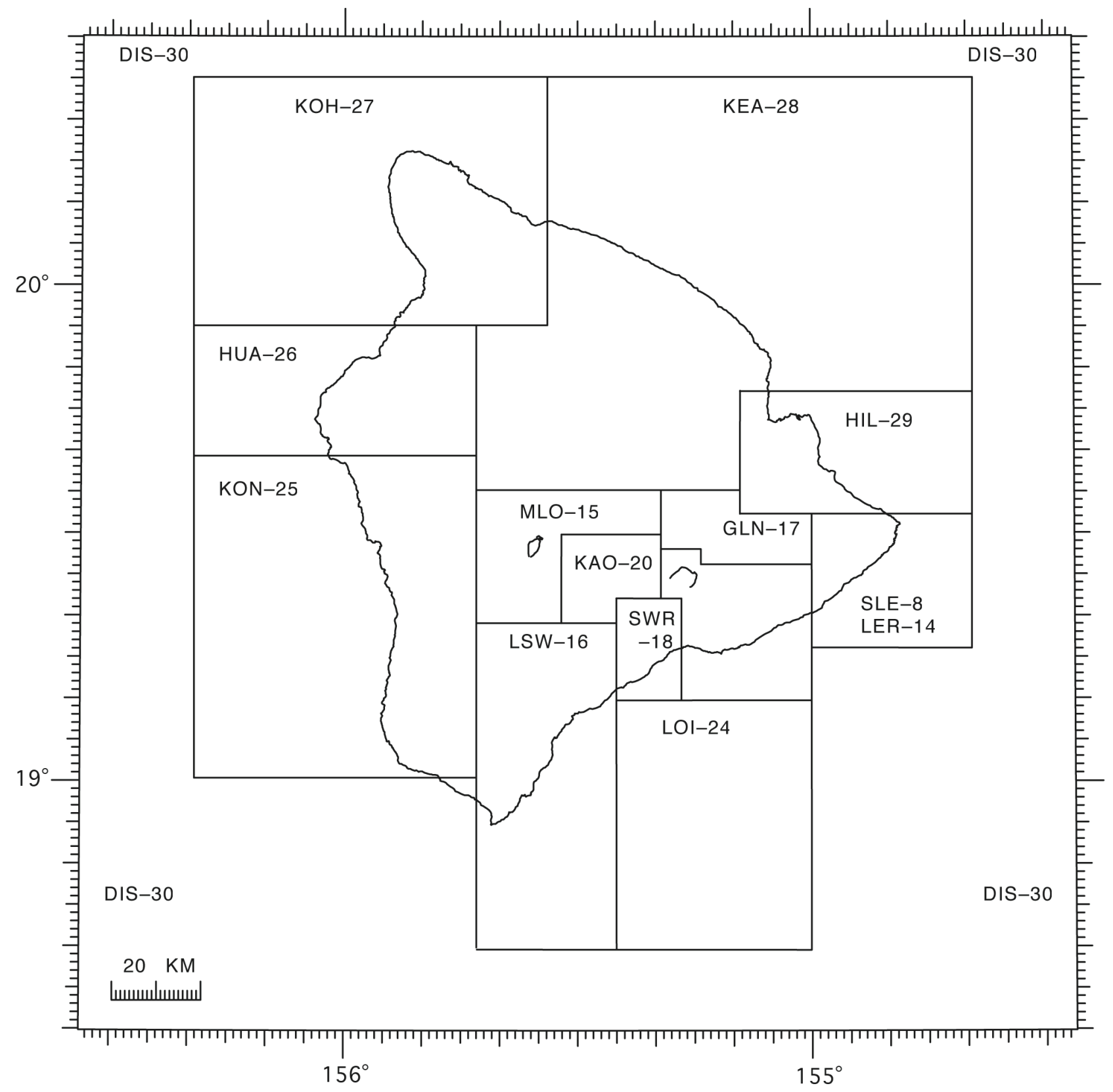

Figure 9. Regions for earthquake classification for Hawai' $i$ Island. Depth range and coordinate boundaries for each region are described in table 3 . 


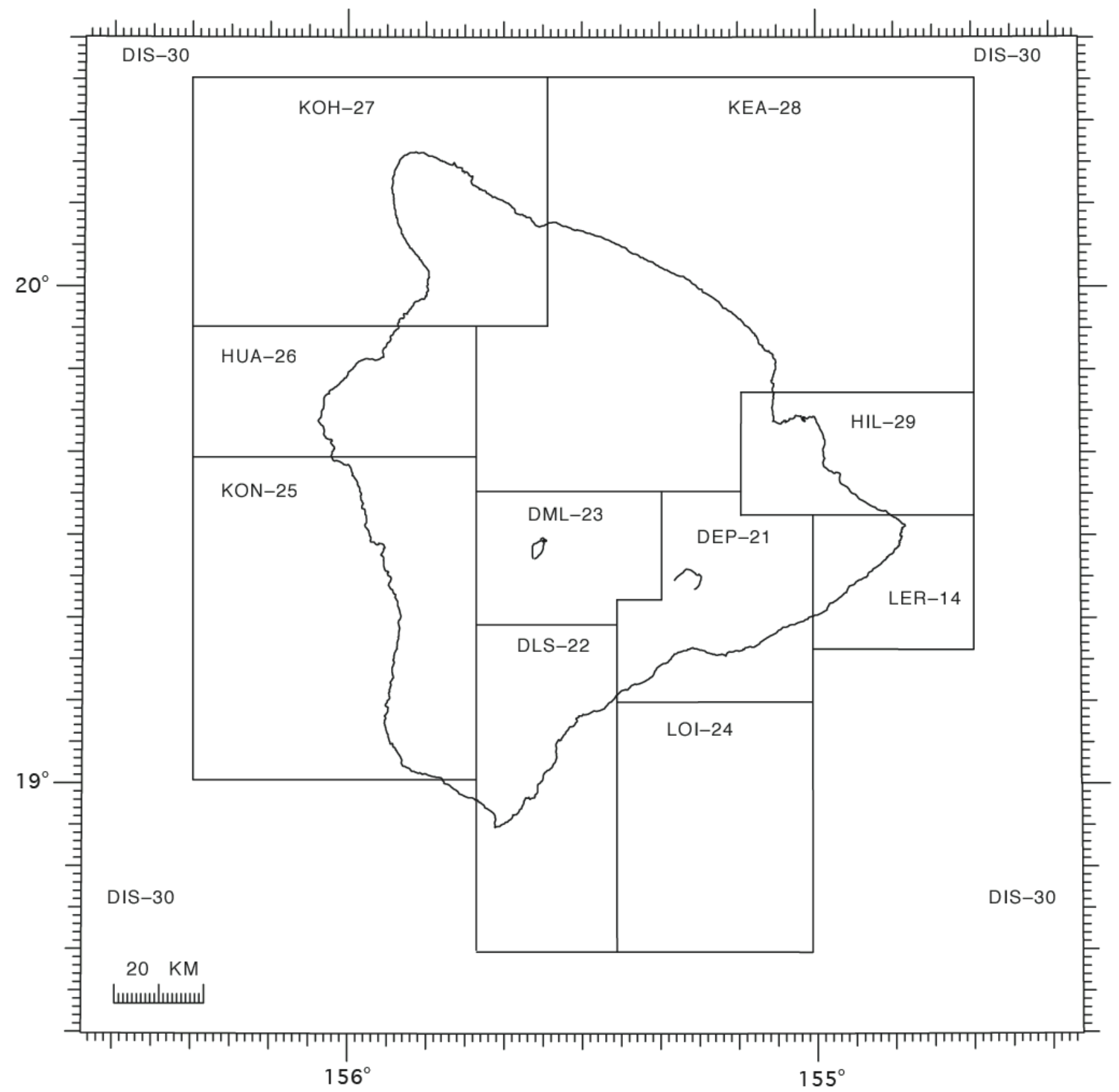

Figure 10. Regions for earthquake classification for the Hawai'i Island. Depth range and coordinate boundaries for each region are described in table 3. 


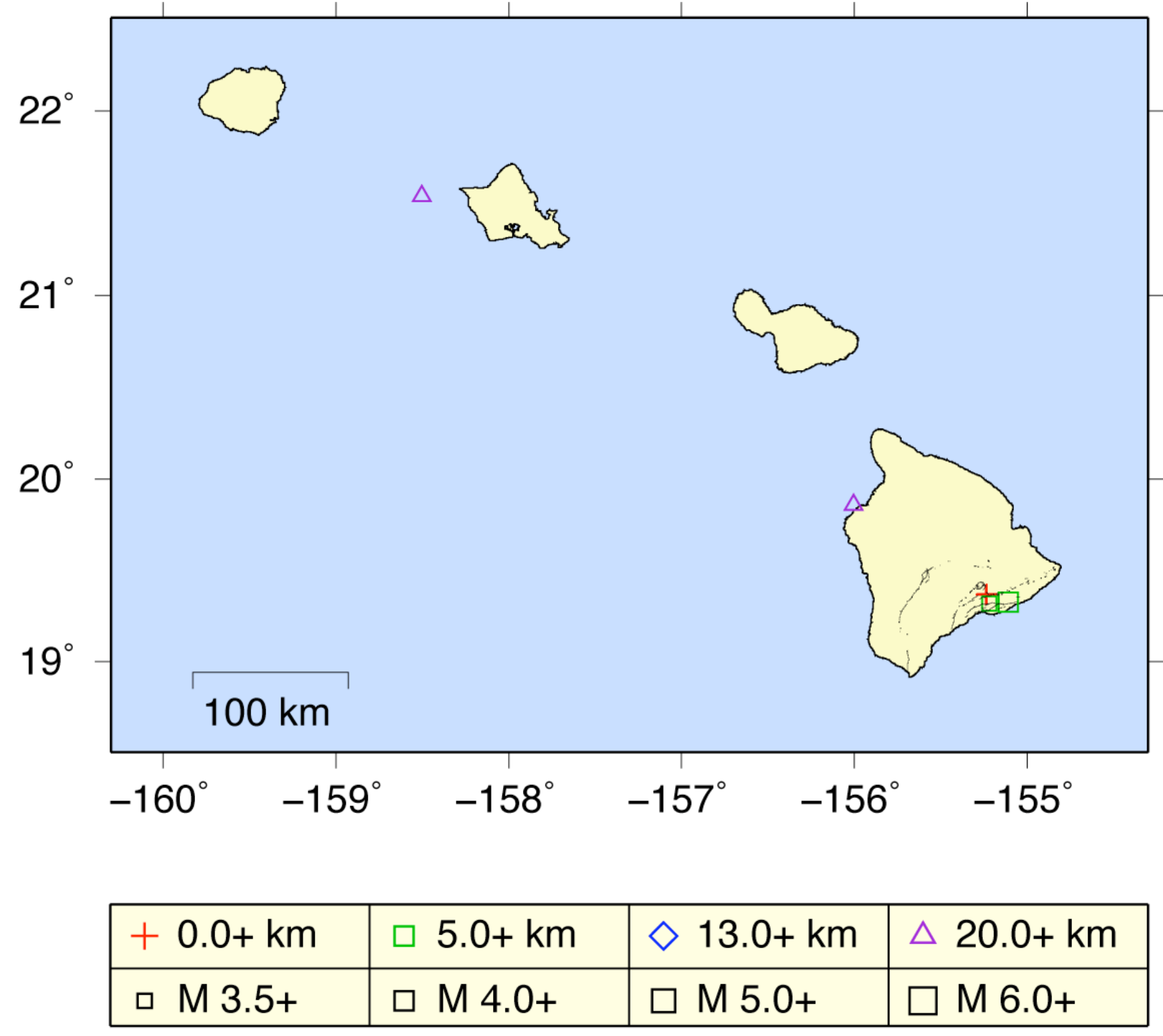




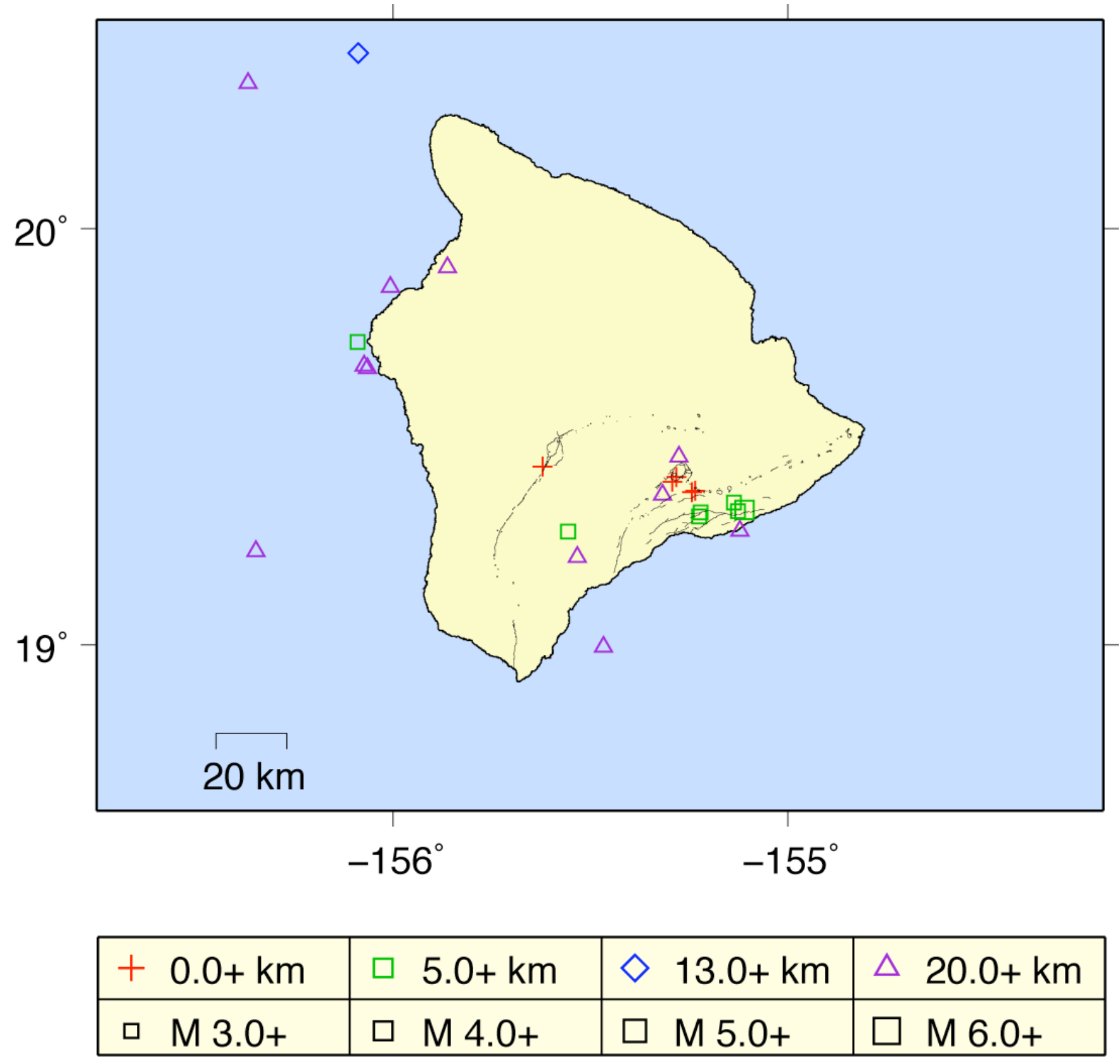




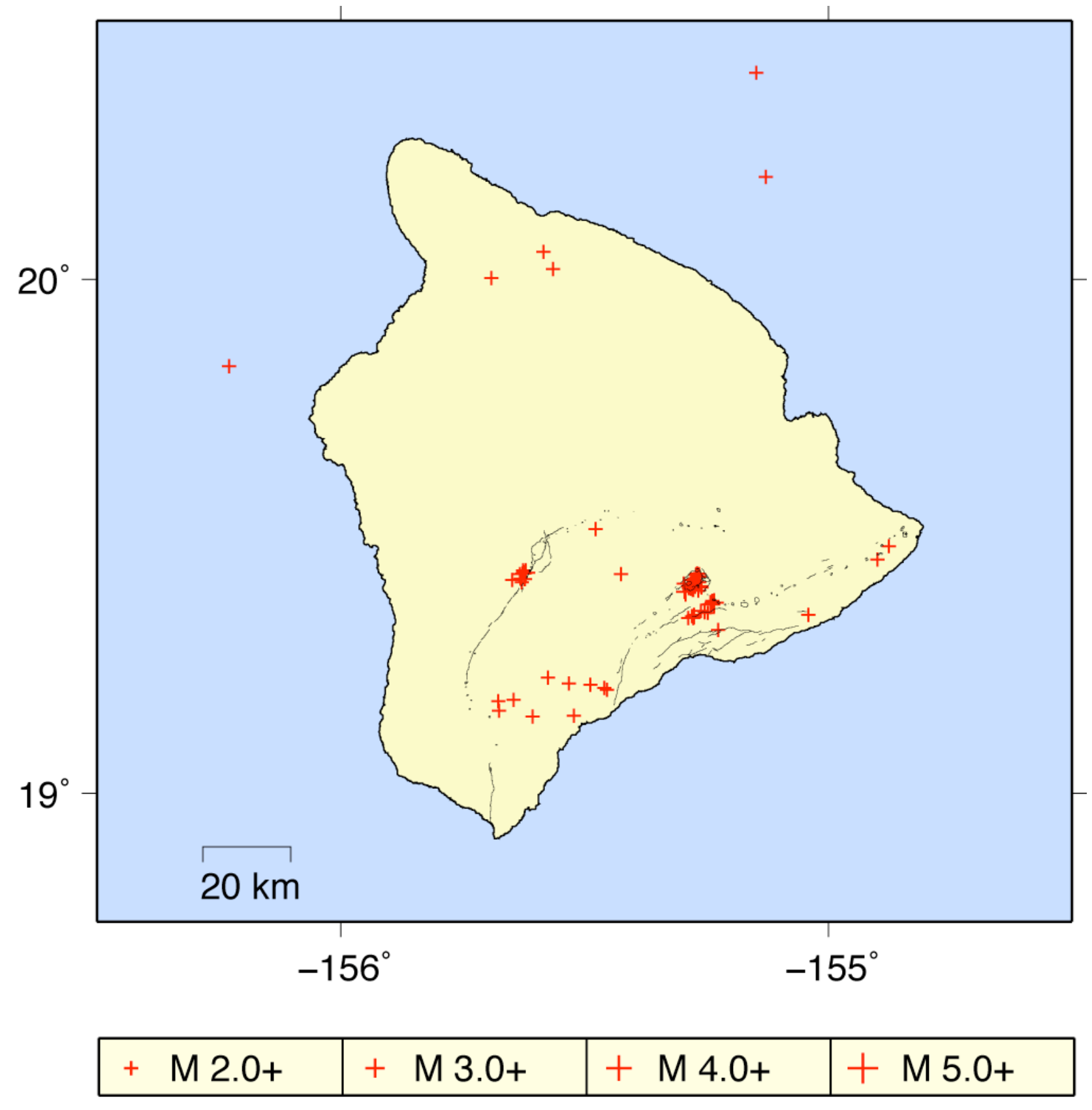

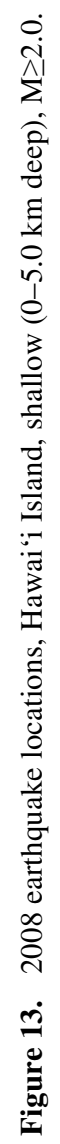




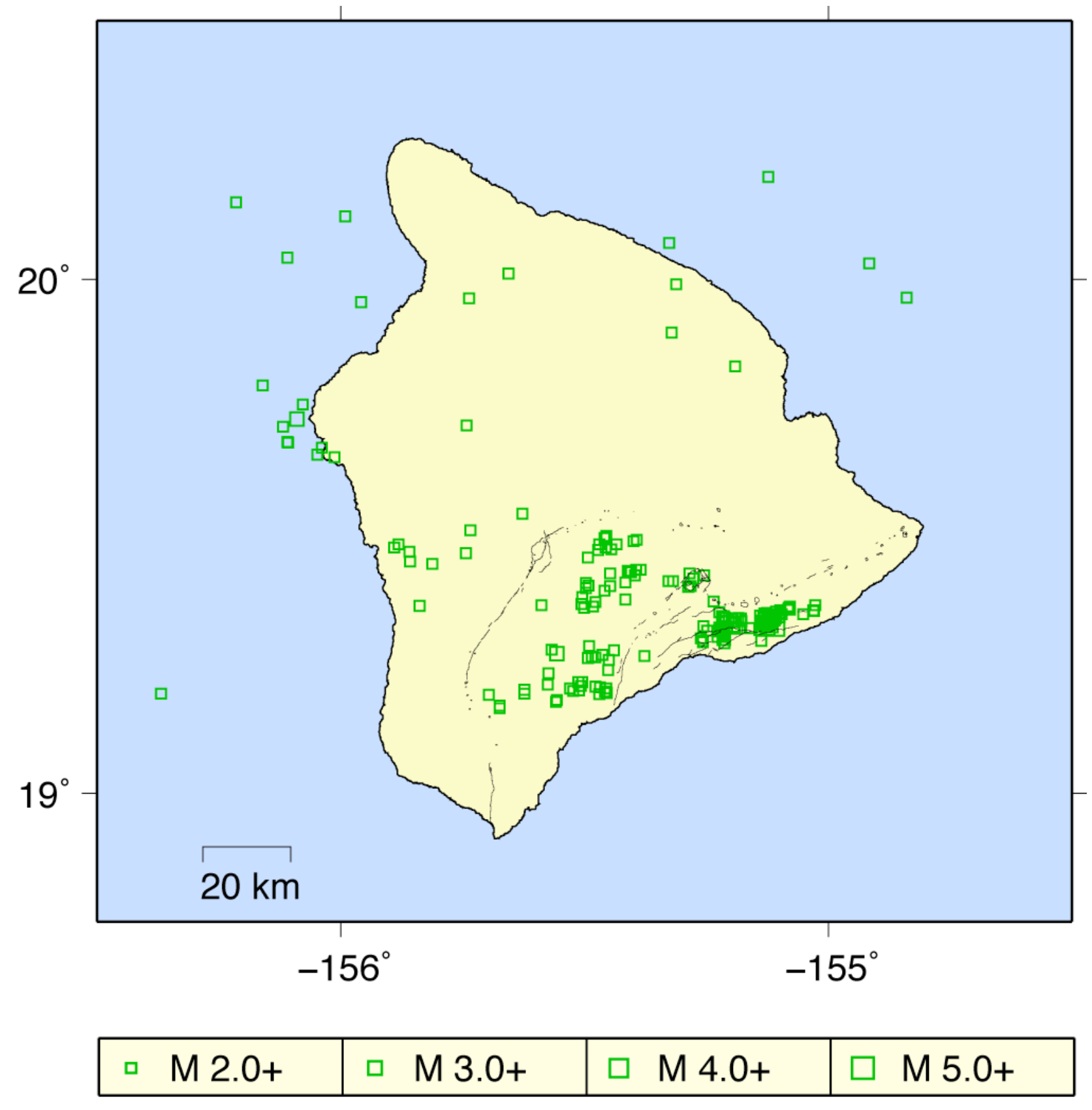

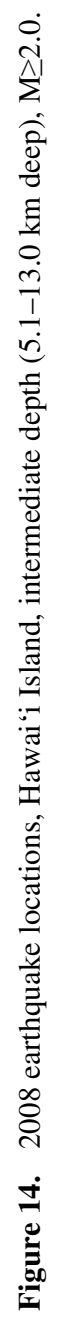




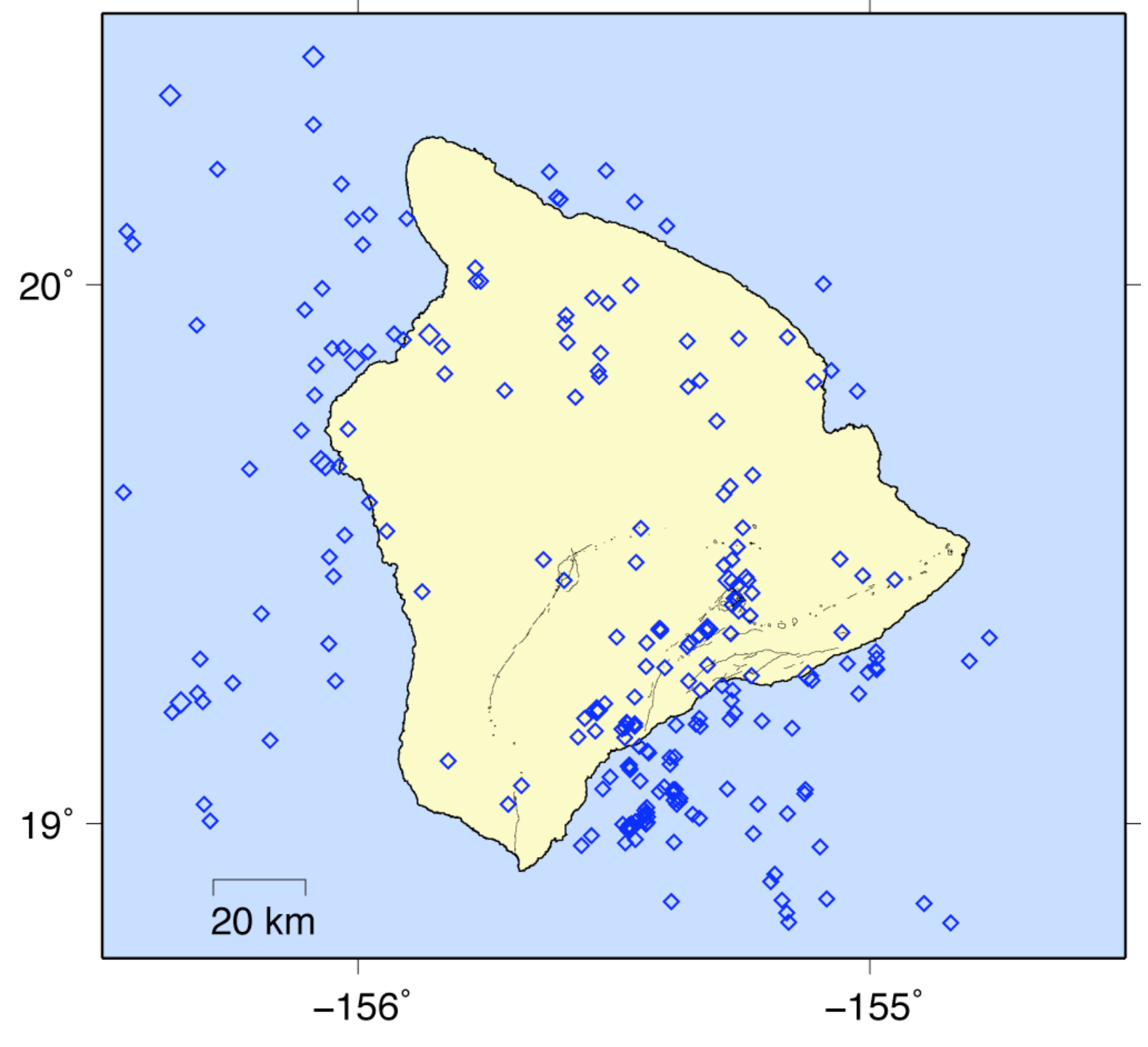

\begin{tabular}{|l|l|l|l|}
\hline$\diamond \mathrm{M} 2.0+$ & $\diamond \mathrm{M} 3.0+$ & $\diamond \mathrm{M} 4.0+$ & $\diamond \mathrm{M} 5.0+$ \\
\hline
\end{tabular}



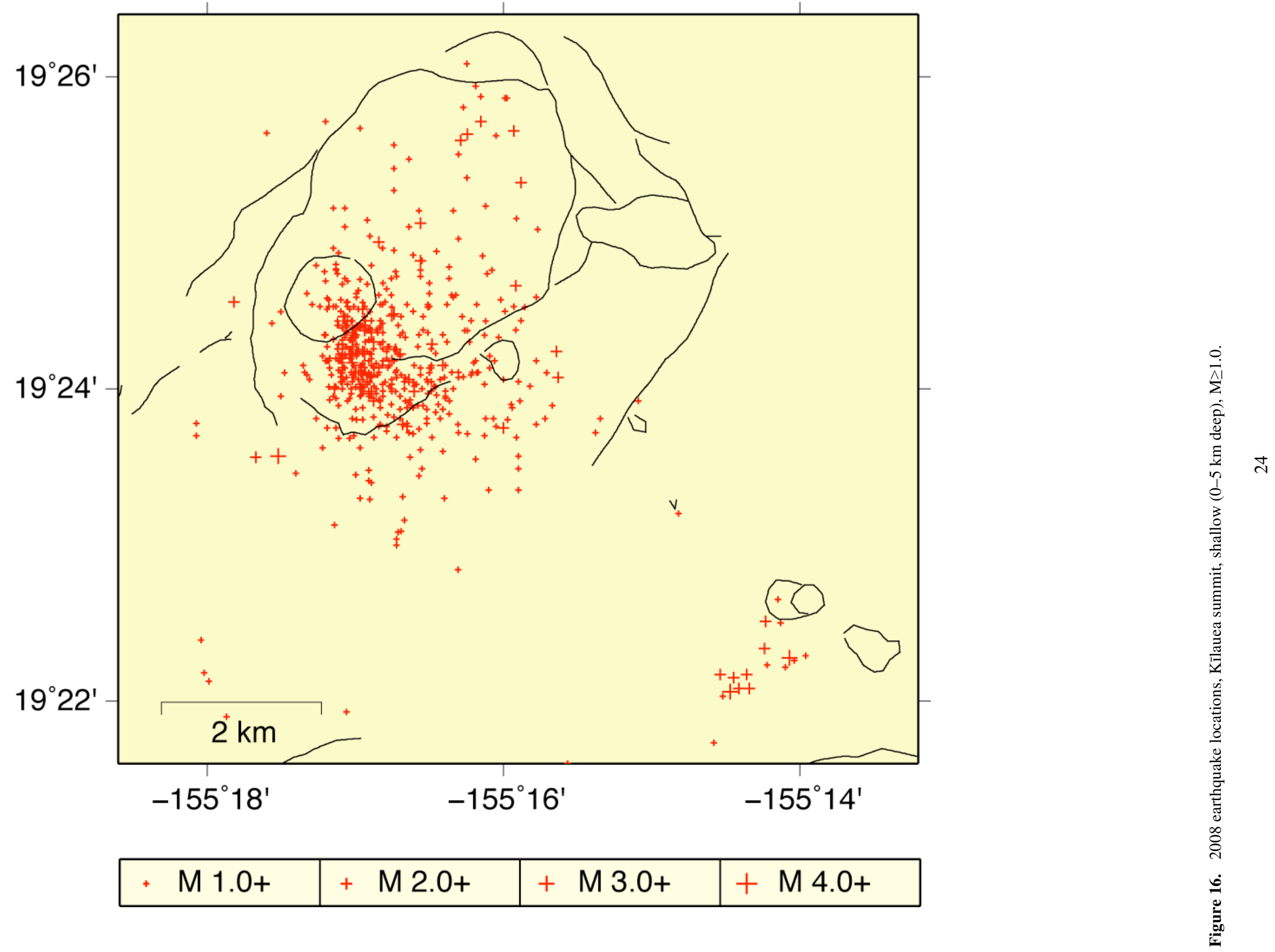


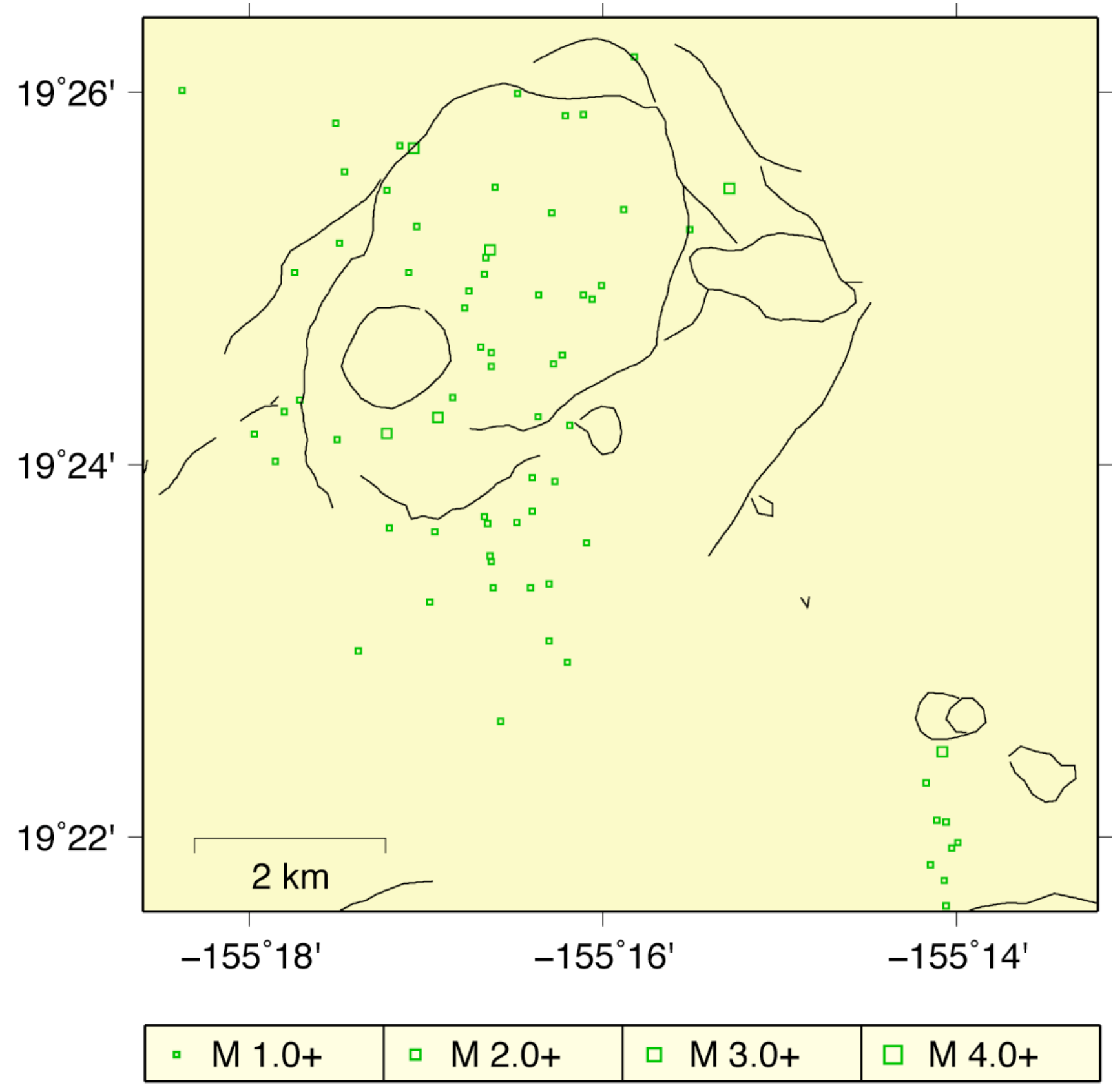




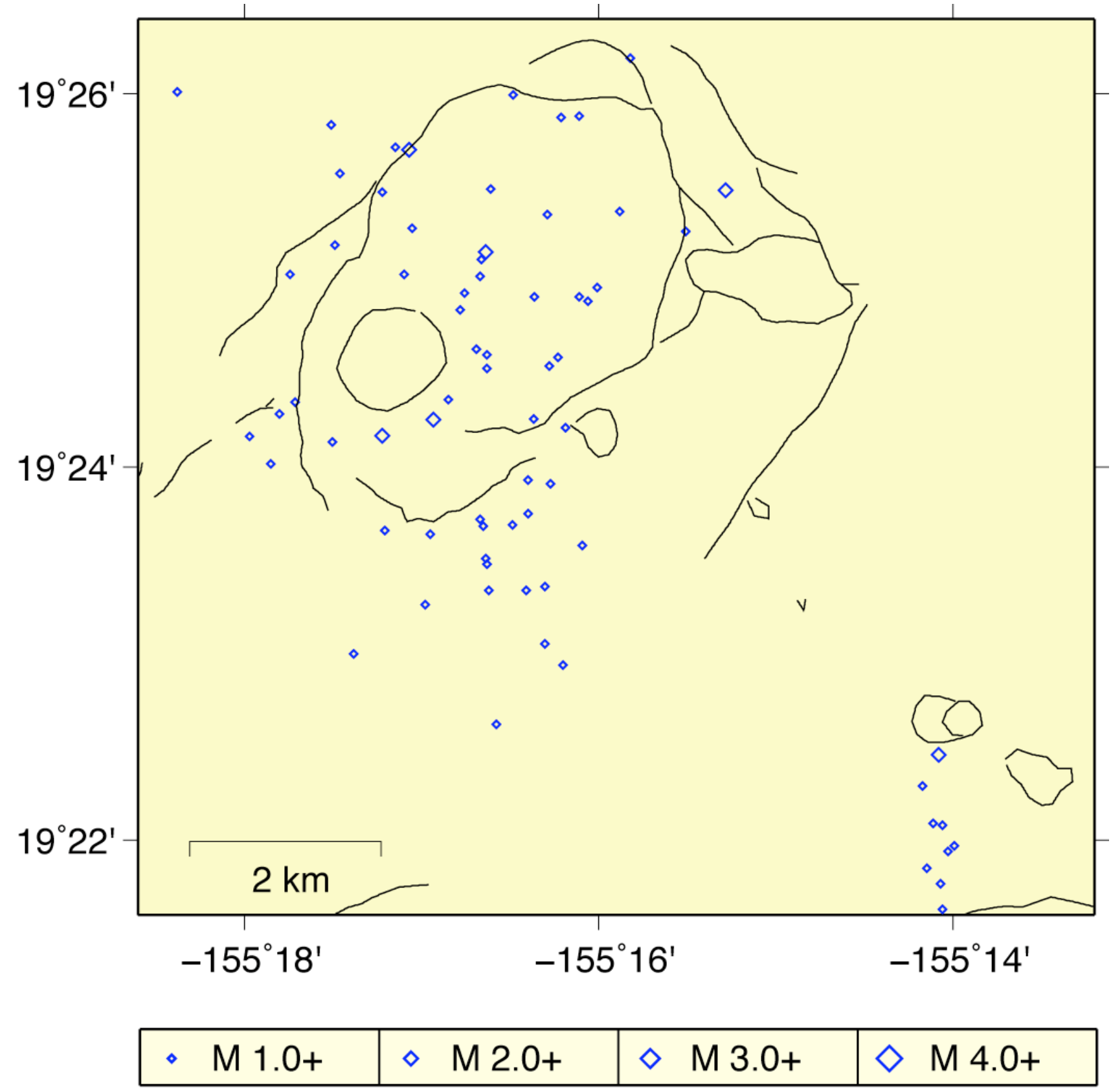




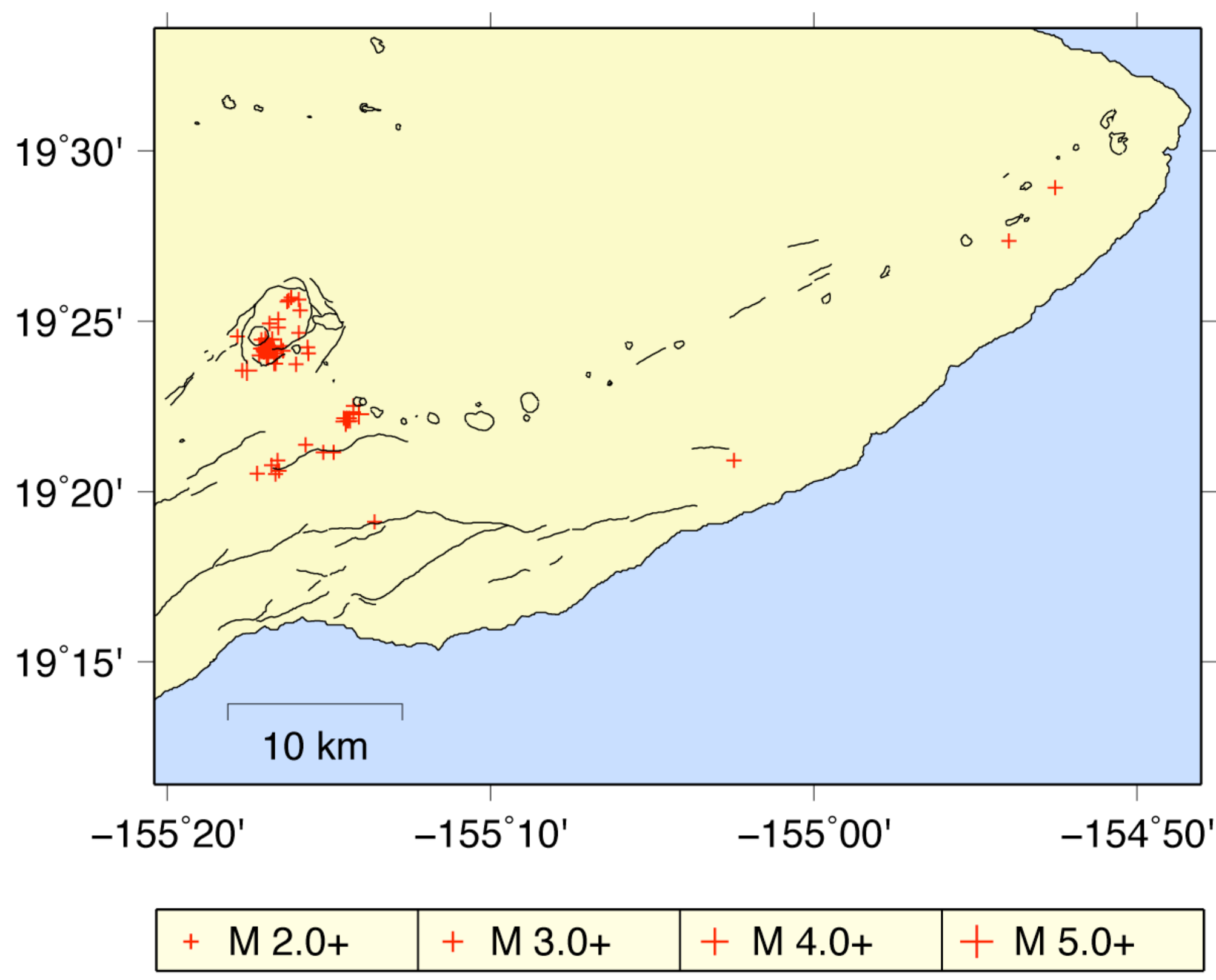




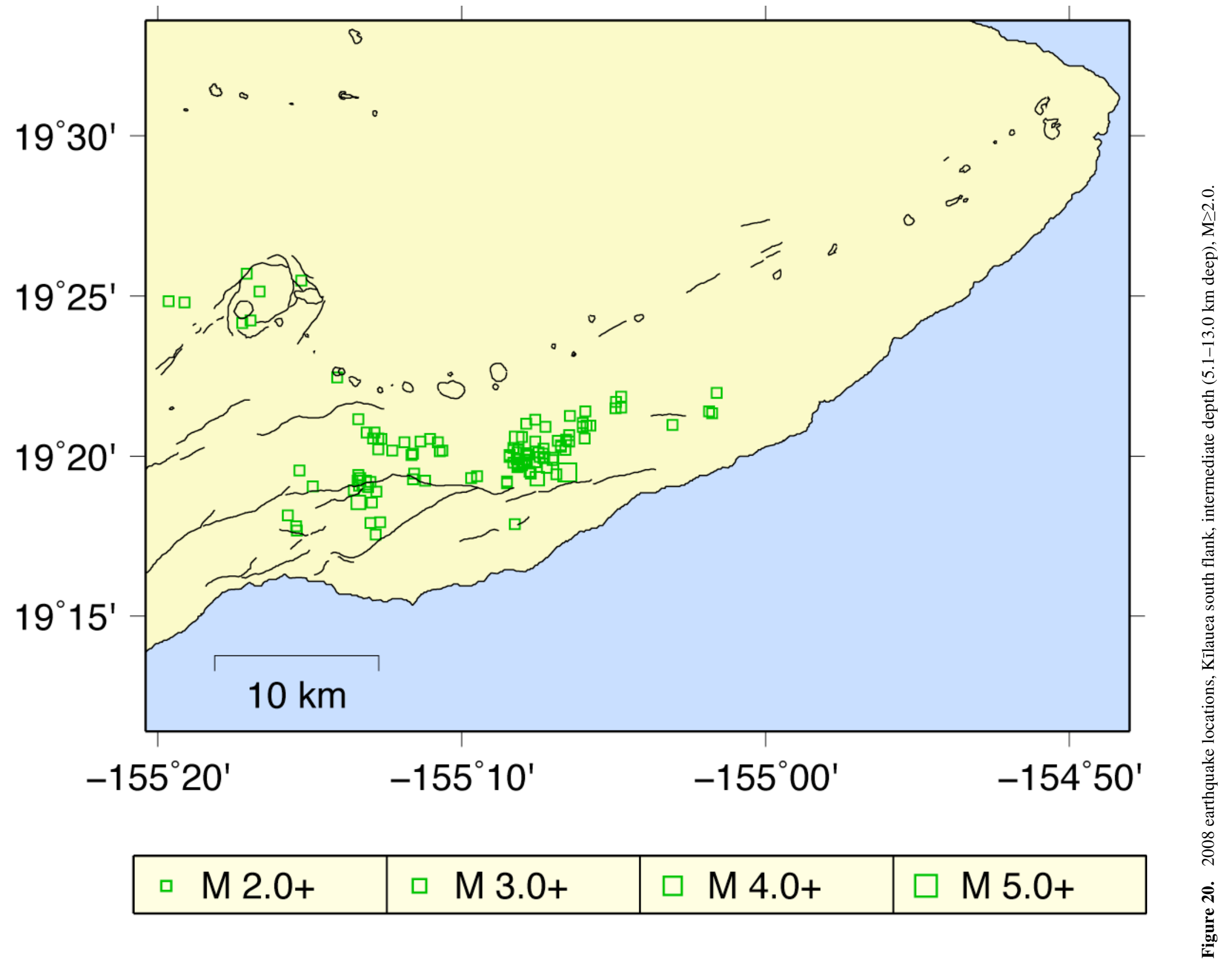




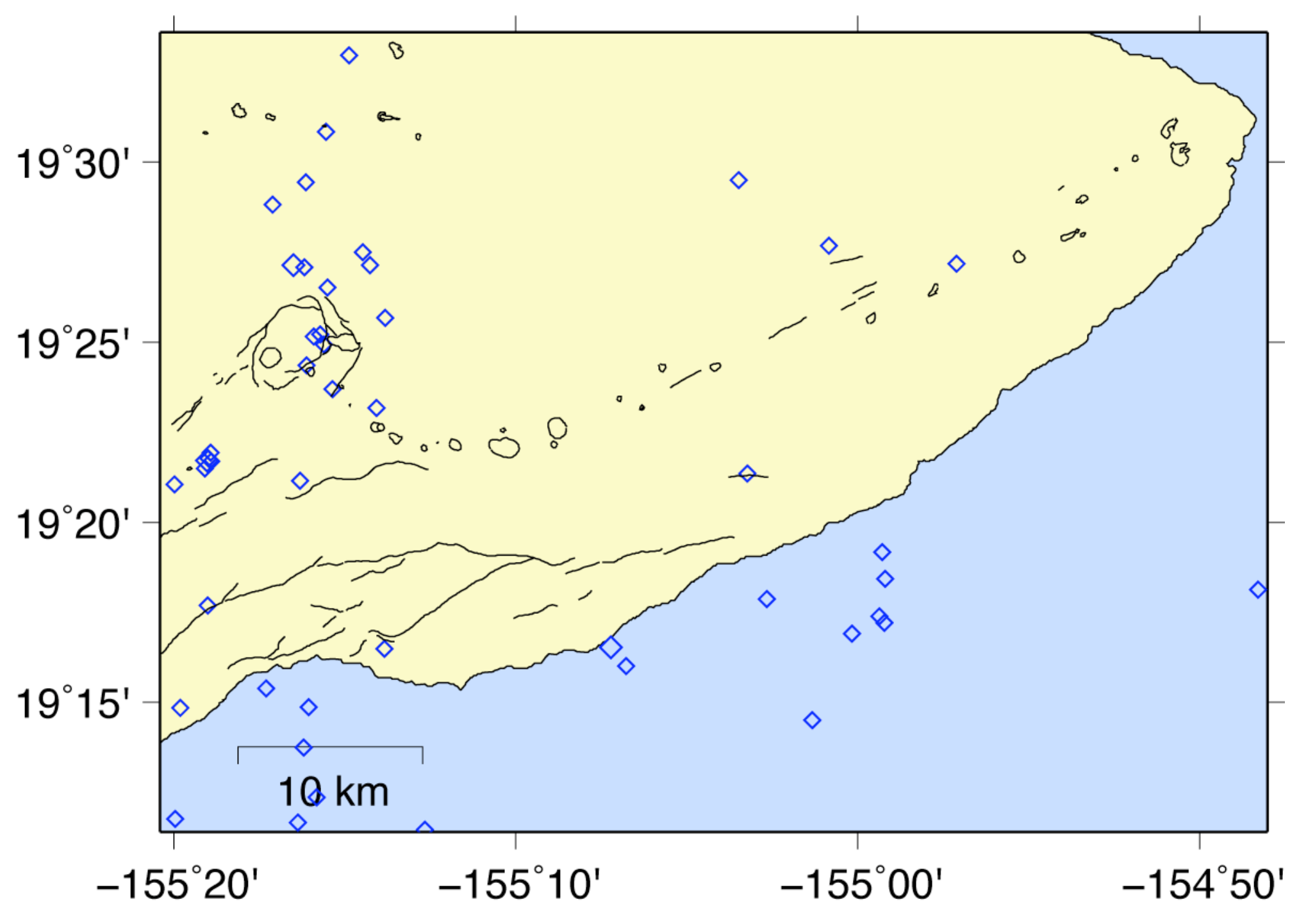




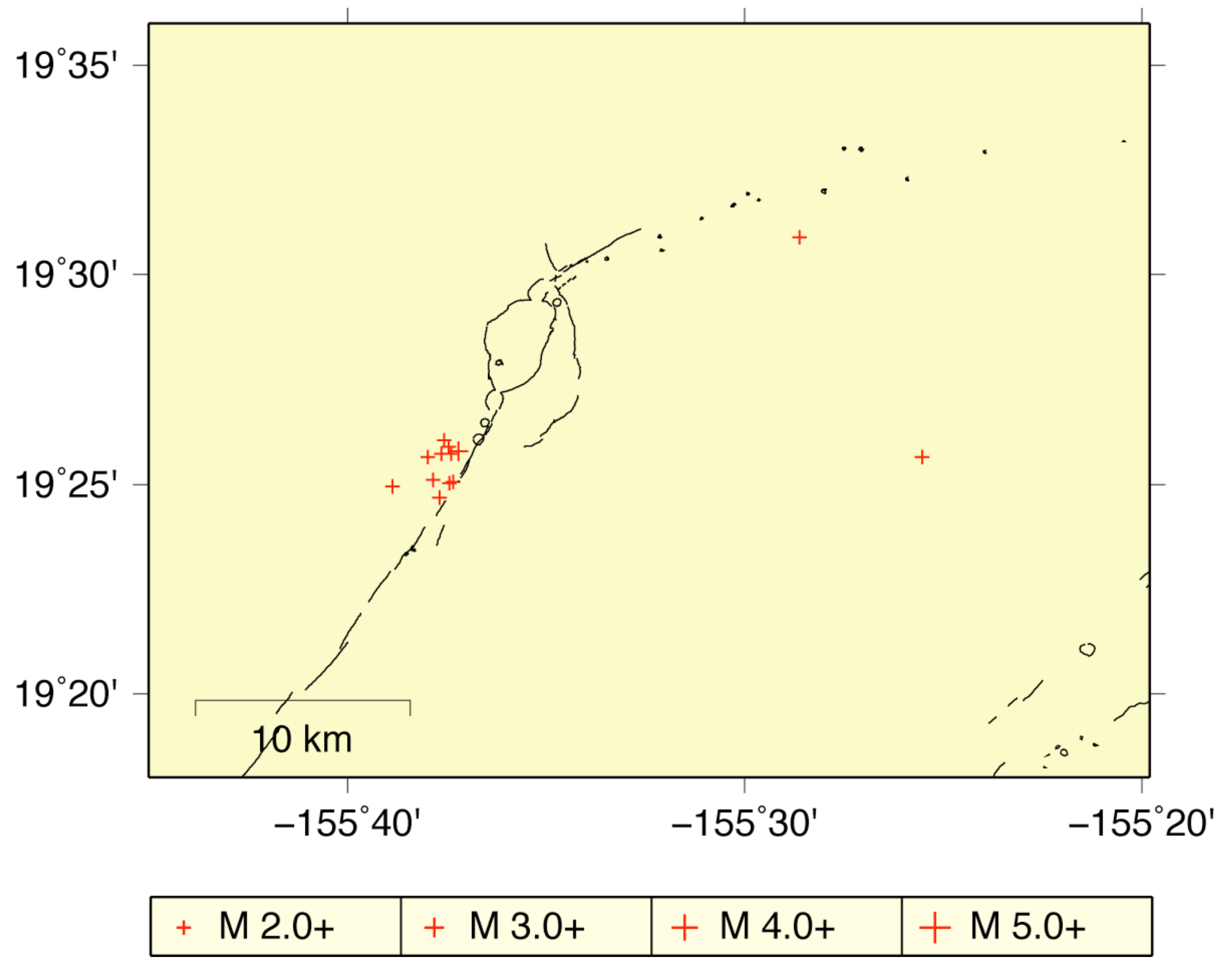




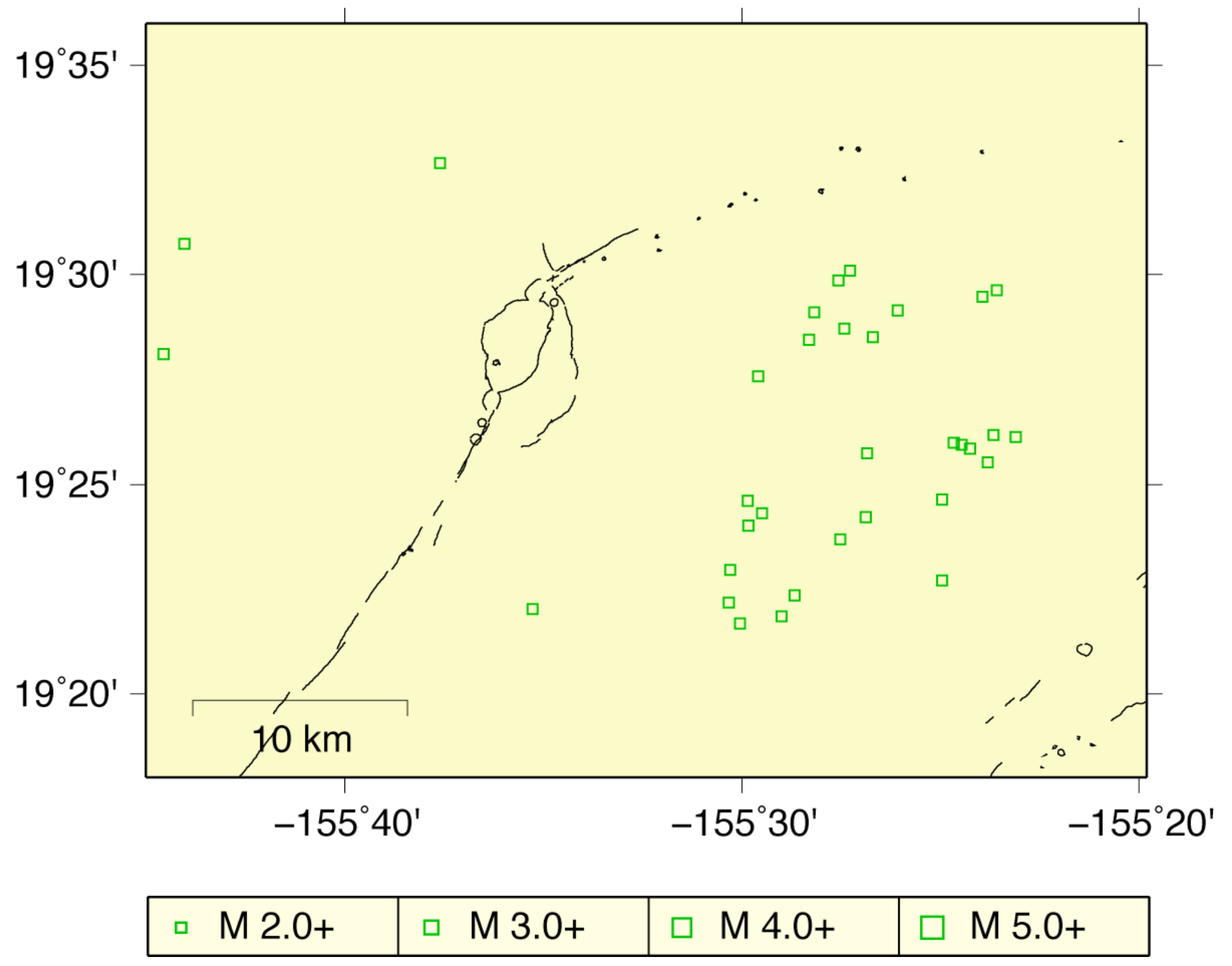




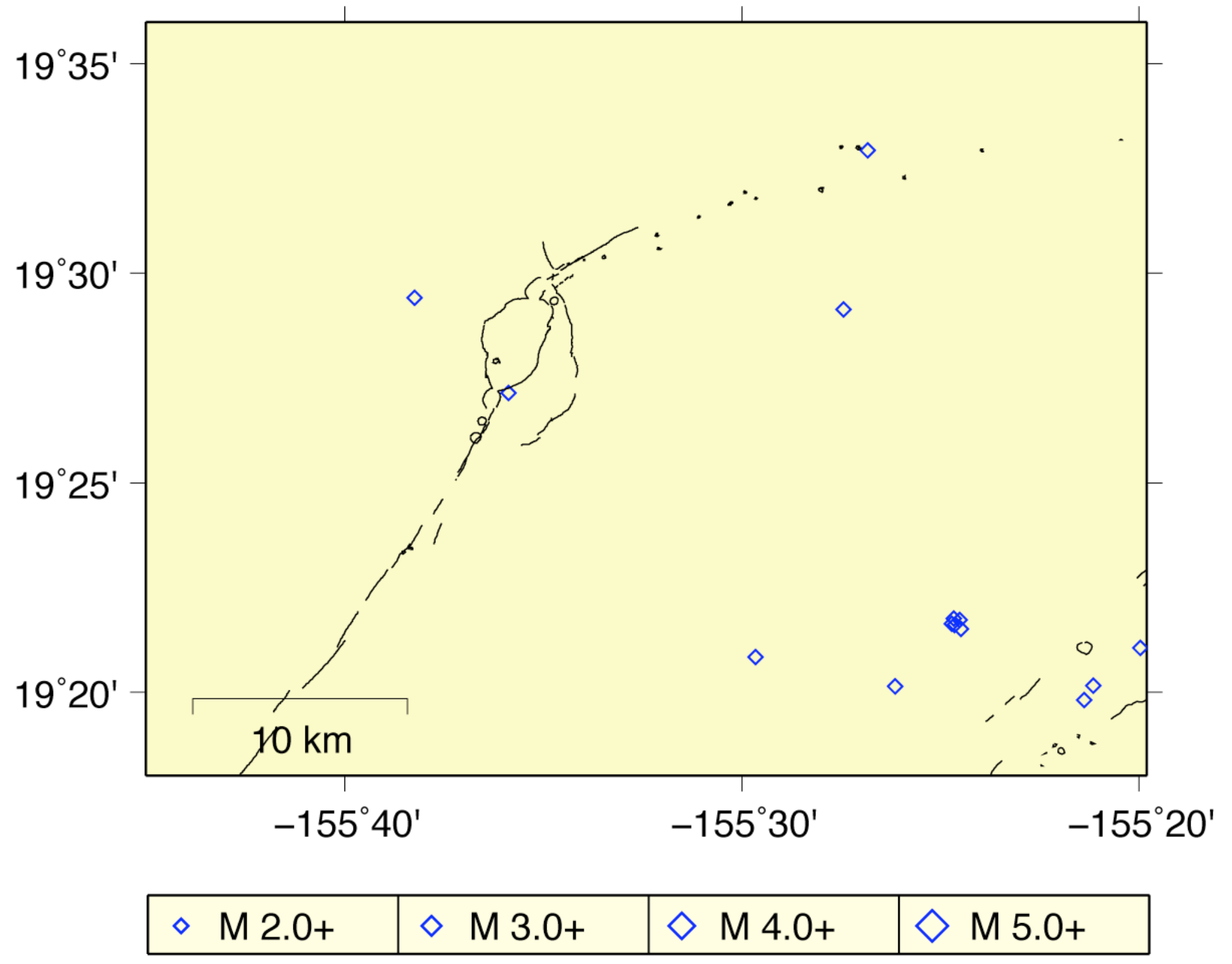


Table 4. Chronological list of the 3,089 selected events successfully located in CUSP during 2008. Summary data files are available online at http://www.ncedc.org/anss/catalog-search.html/ (last accessed 09/21/2009). For each event, the following data are presented.

ORIGIN_-TIME in Hawaiian Standard Time: date, hour (HR), minute (MN), and second (SEC).

EPICENTER — in degrees and minutes of north latitude (LAT N) and west longitude (LON W) in Old Hawaiian Datum.

DEPTH—depth of focus in kilometers.

NRD—number of $\mathrm{P} \& \mathrm{~S}$ readings with final weights $>0.1$.

NS-number of S readings with final weights $>0.1$.

RMS SEC — root mean square travel time residuals, in seconds.

ERH $\mathrm{km}$ — standard error of the epicenter, in kilometers.

ERZ $\mathrm{km}$ - standard error of depth of focus, in kilometers.

LOC REMKS—remarks, three-letter code for geographic location of events. See Figures 7-10 for location of mnemonic code. Additional one-letter codes have the following meanings:

F felt,

L long-period character,

$\mathrm{T}$ associated with harmonic tremor,

B quarry or other blast,

\# location program had a convergence problem, which usually means that the depth may be unreliable, and

- $\quad$ depth was held fixed.

PREF MAG-The preferred magnitude chosen from the available magnitudes.

Preference is set as:

$\mathrm{X}$ amplitude magnitude, if none,

D duration magnitude Develocorder equivalent, if none,

$\mathrm{U}$ external magnitude, usually calculated from drum records or from an external source.

AZ GAP — Largest azimuthal gap in degrees between azimuthally adjacent stations.

MIN DS-Distance to the nearest station, in kilometers. 
Table 4.

---ORIGIN TIME (HST)-- -LAT N-- --LON W-- DEPTH N N RMS ERH ERZ LOC PREF AZ MIN YEAR MON DA HRMN SEC DEG MIN DEG MIN KM RD S SEC KM KM REMKS MAG GAP DS

$\begin{array}{lllllllllllllllllll}2008 & \text { JAN } & 1 & 0310 & 54.53 & 19 & 7.47 & 155 & 22.86 & 48.71 & 39 & 6 & .07 & .8 & 1.2 & \text { LOI } & 2.0 \times & 188 & 10\end{array}$ $\begin{array}{lllllllllllllllllll}2008 & \text { JAN } & 1 & 0337 & 2.03 & 19 & 28.09 & 155 & 35.25 & 10.01 & 30 & 5 & .15 & .5 & .8 & \text { MLO } & 1.9 \mathrm{X} & 58 & 0\end{array}$ $\begin{array}{lllllllllllll}2008 & 0 & 0\end{array}$

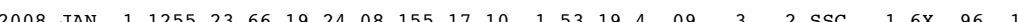

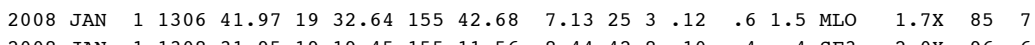

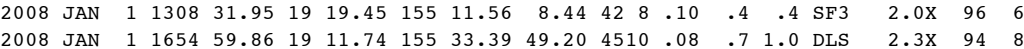

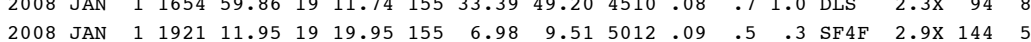

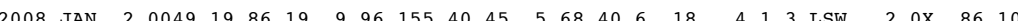

$\begin{array}{lllllllllllllllllll}2008 & & & \end{array}$

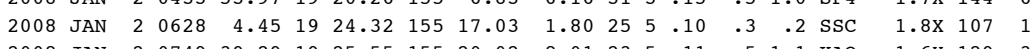

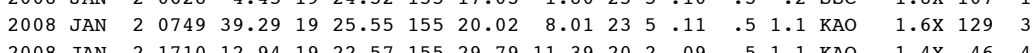

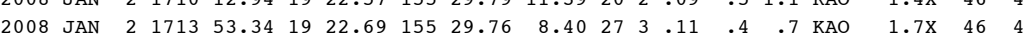

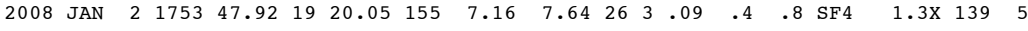

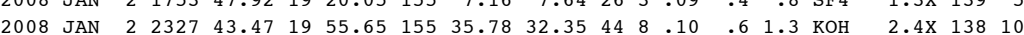

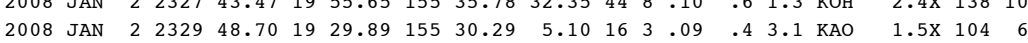
2008 JAN $30127 \begin{array}{lllllllllllllll}03.56 & 19 & 24.28 & 155 & 16.99 & 1.81 & 21 & 4 & .05 & .3 & .2 & \text { SSC } & 1.9 \times & 108 & 1\end{array}$

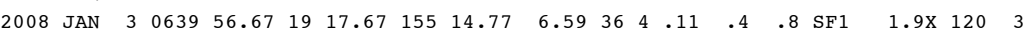

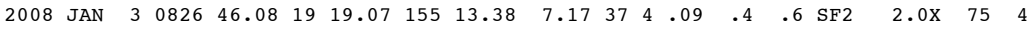

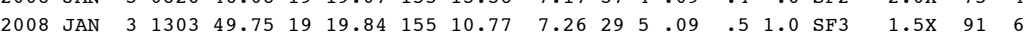

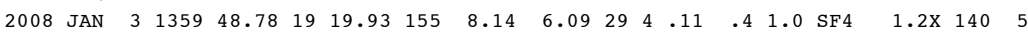

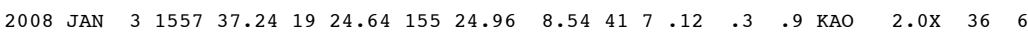

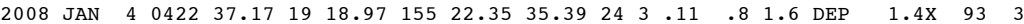

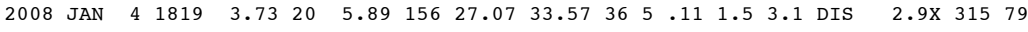

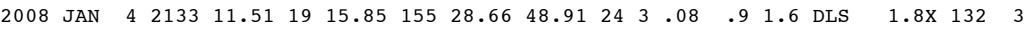

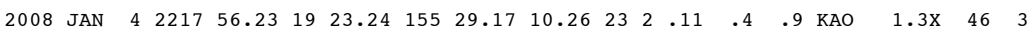

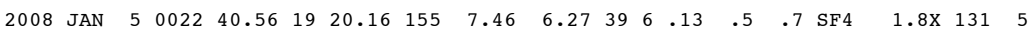

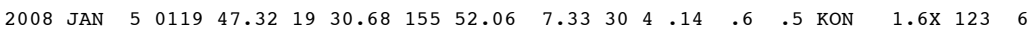

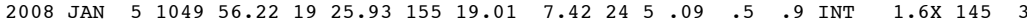
$\begin{array}{lllllllllllllllllll}2008 & \text { JAN } & 6 & 0808 & 4.18 & 19 & 28.89 & 155 & 53.08 & 7.41 & 31 & 4 & .13 & .6 & .7 & \text { KON } & 1.7 \times & 106 & 4\end{array}$

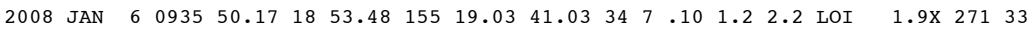
2008 JAN $6 \begin{array}{lllllllllllllllll}1854 & 4.91 & 19 & 12.07 & 155 & 31.17 & 9.03 & 23 & 5 & .12 & .4 & 1.1 & \text { LSW } & 1.6 \times & 153 & 6\end{array}$

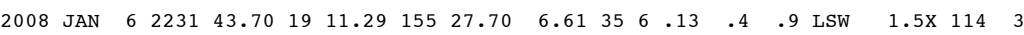

$\begin{array}{llllllllllllllllllll}2008 & \text { JAN } & 7 & 0233 & 43.07 & 19 & 26.96 & 155 & 24.14 & 11.08 & 27 & 5 & .09 & .4 & .8 & \text { KAO } & 1.3 \mathrm{X} & 89 & 5\end{array}$

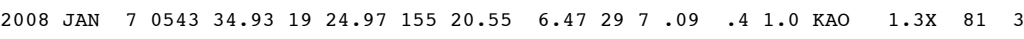
2008 JAN $7 \begin{array}{llllllllllllllll}0704 & 37.02 & 19 & 23.00 & 155 & 17.38 & 12.03 & 39 & 9 & .08 & .4 & .4 & \text { INT } & 1.7 \mathrm{X} & 42 & 1\end{array}$

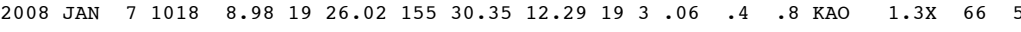
$\begin{array}{lllllllllllllllllll}2008 & \text { JAN } & 7 & 1652 & 0.41 & 19 & 21.72 & 155 & 29.89 & 8.64 & 35 & 4 & .10 & .3 & .8 & \text { KAO } & 1.5 \mathrm{X} & 49 & 4\end{array}$

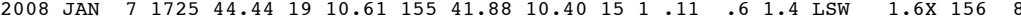

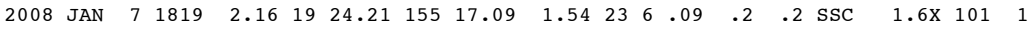

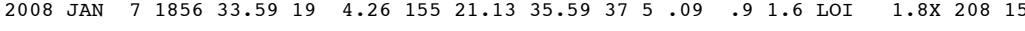
2008 JAN $7 \begin{array}{lllllllllllllllll}7 & 2112 & 55.33 & 19 & 22.51 & 155 & 14.85 & 33.41 & 41 & 6 & .11 & .7 & 1.0 & \text { DEP } & 1.8 \mathrm{X} & 53 & 2\end{array}$

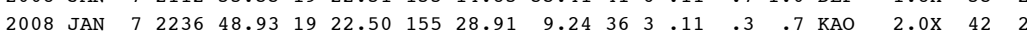

---ORTGIN TIME (HST)-- -LAT N-- --LON W-- DEPTH N N RMS ERH ERZ LOC PREF AZ MIN YEAR MON DA HRMN SEC DEG MIN DEG MIN KM RD S SEC KM KM REMRS MAG GAP DS

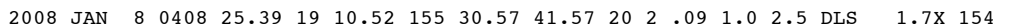
$\begin{array}{lllllllllllllllllll}2008 & \text { JAN } & 8 & 0441 & 0.66 & 19 & 22.21 & 155 & 26.48 & 10.68 & 19 & 2 & .11 & .5 & .9 & \text { KAO } & 1.2 \mathrm{x} & 48 & 2\end{array}$

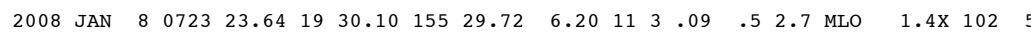

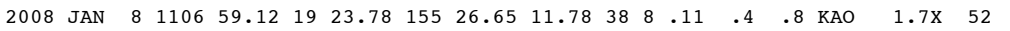

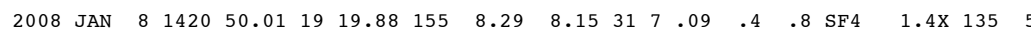

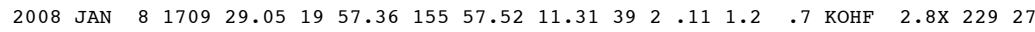

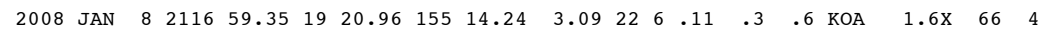
$\begin{array}{lllllllllllllllllll}2008 & \text { JAN } & 8 & 2127 & 55.21 & 19 & 24.53 & 155 & 16.94 & 1.91 & 19 & 5 & .13 & .3 & .3 & \text { SSC } & 1.4 \times & 121 & 1\end{array}$

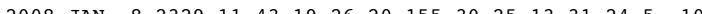

$\begin{array}{llllllllllllllllll}2008 & \text { JAN } & 9 & 0013 & 23.25 & 19 & 21.02 & 155 & 21.69 & 32.59 & 34 & 7 & .08 & .6 & 1.0 & \text { DEP } & 1.4 \mathrm{X} & 63\end{array}$

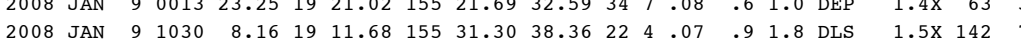

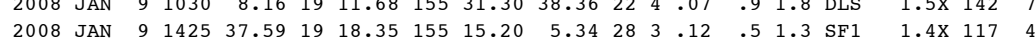

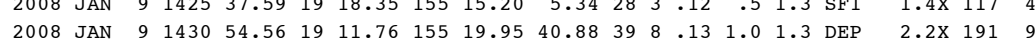
$\begin{array}{llllllllllllllllll}2008 & 008 \text { JAN } & 9 & 1432 & 17.25 & 19 & 11.08 & 155 & 20.36 & 49.85 & 41 & 6 & .10 & .9 & 1.2 & \text { DEP } & 2.3 \times & 197\end{array}$

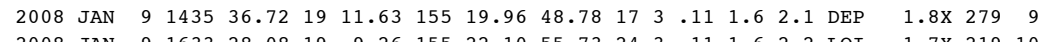

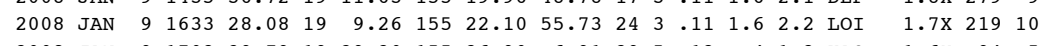

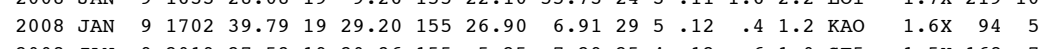

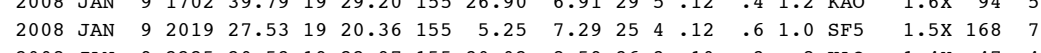

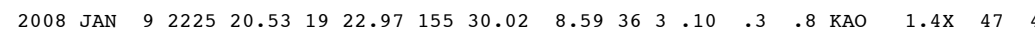

$\begin{array}{llllllllllllllllll}2008 \text { JAN } & 9 & 2242 & 28.64 & 19 & 19.71 & 155 & 8.15 & 7.80 & 26 & 4 & .07 & .4 & .6 & \text { SF } 4 & 1.3 \times & 117 & 4\end{array}$

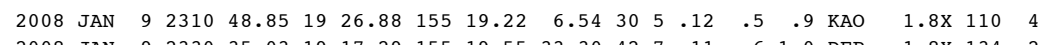

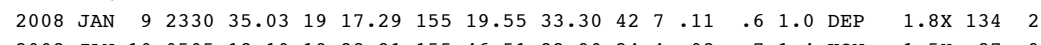

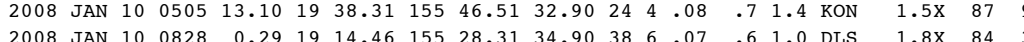

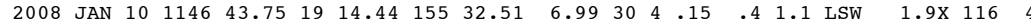

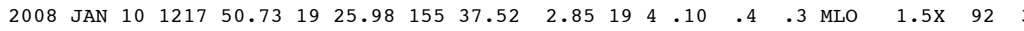

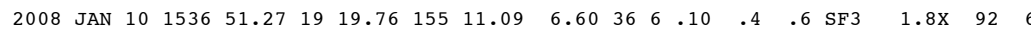
$\begin{array}{lllllllllllllllll}2008 & \text { JAN } 11 & 0524 & 16.17 & 19 & 15.85 & 155 & 24.40 & 35.53 & 35 & 5 & .12 & .8 & 1.2 & \text { DEP } & 1.8 \mathrm{X} & 82\end{array}$

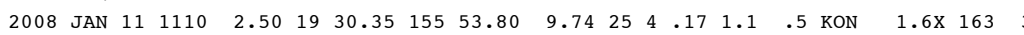

$\begin{array}{llllllllllllllllll}2008 & \text { JAN } 11 & 1802 & 5.04 & 19 & 18.16 & 155 & 30.31 & 9.10 & 32 & 7 & .09 & .3 & .9 & \text { LSW } & 1.5 \mathrm{x} & 63 & 6\end{array}$

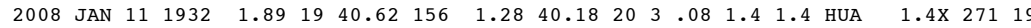

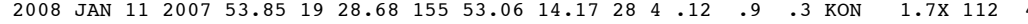

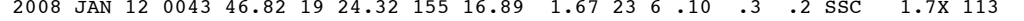

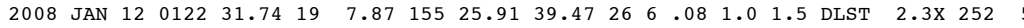

$\begin{array}{lllllllllllllllll}2008 & \text { JAN } 12 & 1334 & 8.06 & 19 & 30.09 & 155 & 30.21 & 8.98 & 19 & 4 & .11 & .4 & 1.5 & \text { MLO } & 1.7 \times & 107\end{array}$

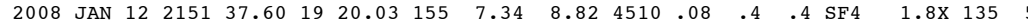

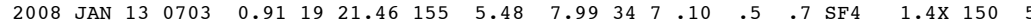

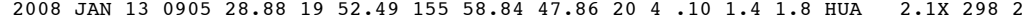

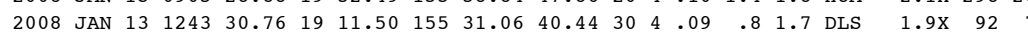

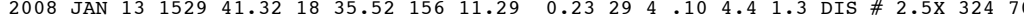

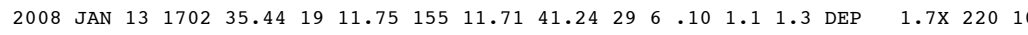

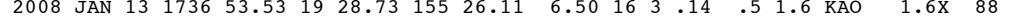

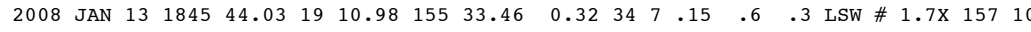

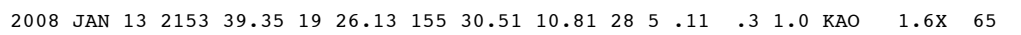


---ORIGIN TIME (HST)-- -LAT N-- --LON W-- DEPTH N N RMS ERH ERZ LOC PREF AZ MIN YEAR MON DA HRMN SEC DEG MIN DEG MIN KM RD S SEC KM KM REMKS MAG GAP DS

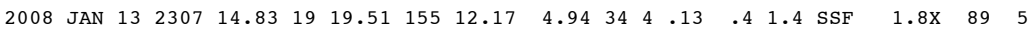

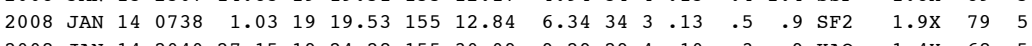
$\begin{array}{lllllllllllll} & 2008 \\ J\end{array}$

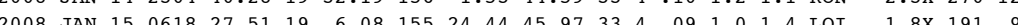

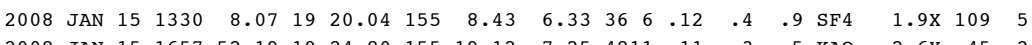

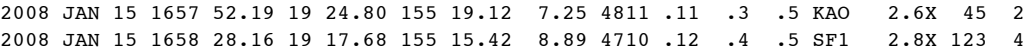

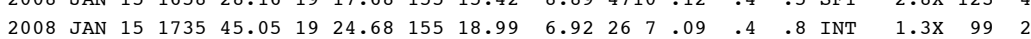
$\begin{array}{lllllllllllllll}2008 & & \end{array}$

$\begin{array}{llllllllllllllllll}2008 & \text { JAN } 16 & 2010 & 35.00 & 19 & 27.08 & 155 & 25.08 & 10.72 & 19 & 3 & .13 & .5 & 1.2 & \text { KAO } & 1.5 \mathrm{X} & 72 & 6\end{array}$

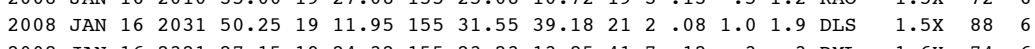

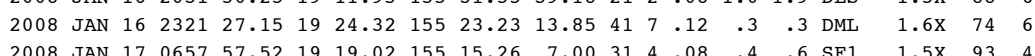

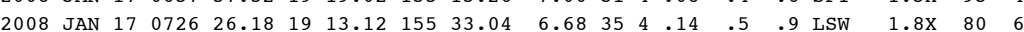

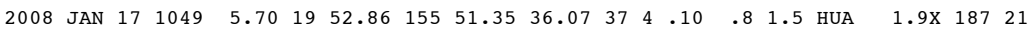
$\begin{array}{lllllllllllllllll}2008 \text { JAN } 17 & 1101 & 32.01 & 19 & 28.51 & 155 & 26.70 & 8.28 & 41 & 7 & .12 & .3 & .8 & \text { KAO } & 2.0 \mathrm{X} & 62 & 6\end{array}$

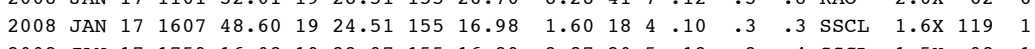

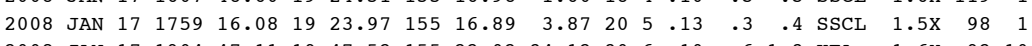

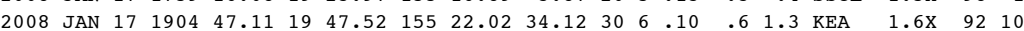

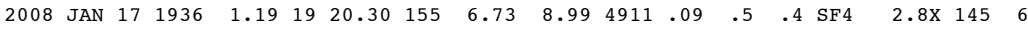

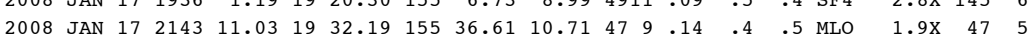

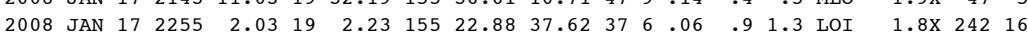

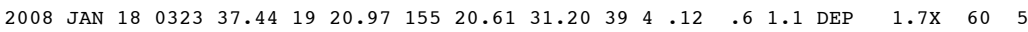

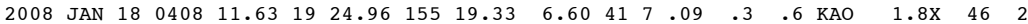

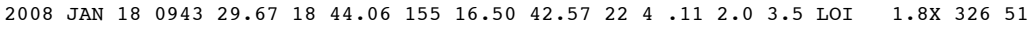

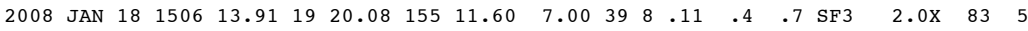

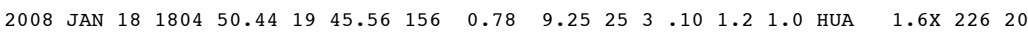
$\begin{array}{lllllllllllllllllll}2008 & \text { JAN } 18 & 2336 & 32.65 & 19 & 30.38 & 155 & 25.34 & 3.03 & 25 & 4 & .16 & .4 & .8 & \text { MLO } & 1.6 \mathrm{X} & 95 & 4\end{array}$

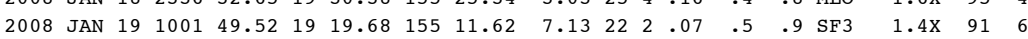

$\begin{array}{llllllllllllllllll}2008 & \text { JAN } 19 & 1514 & 20.47 & 19 & 24.77 & 155 & 19.02 & 6.31 & 36 & 6 & .09 & .3 & .6 & \text { KAO } & 1.8 \mathrm{X} & 67 & 2\end{array}$

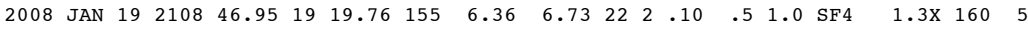

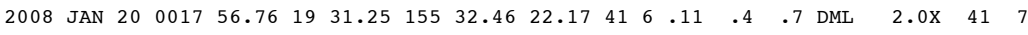

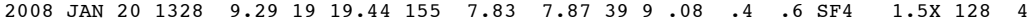

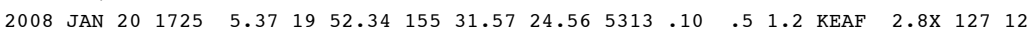

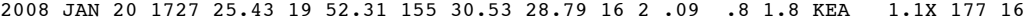

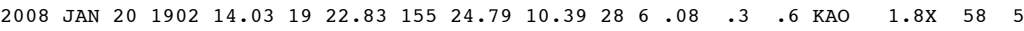

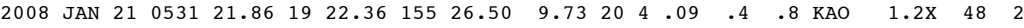

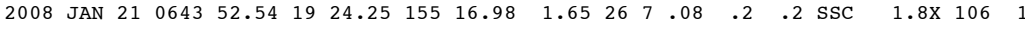

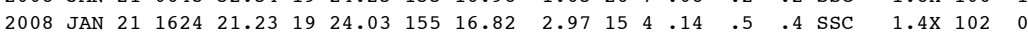

$\begin{array}{lllllllllllllllll}2008 & \text { JAN } 21 & 1740 & 39.30 & 19 & 6.26 & 155 & 28.22 & 30.76 & 4310 & .09 & .7 & 1.1 & \text { DLS } & 2.1 \times & 178 & 6\end{array}$

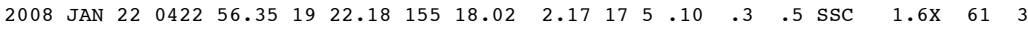

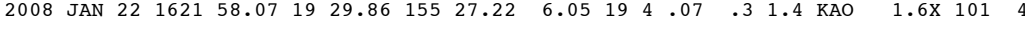

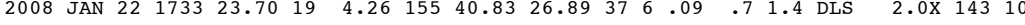

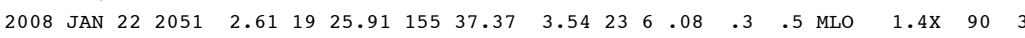

---ORIGIN TTME (HST)-- -LAT N-- --LON W-- DEPTH N N RMS ERH ERZ LOC PREF AZ MIN YEAR MON DA HRMN SEC DEG MIN DEG MIN KM RD S SEC KM KM REMKS MAG GAP DS

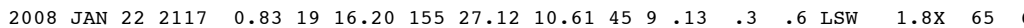
$\begin{array}{llllllllllllllllll}2008 & & & \end{array}$

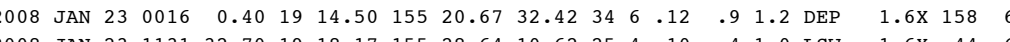
$\begin{array}{llllllllll} & & \end{array}$ $\begin{array}{llllllllllllllllll}2008 & \text { JAN } 23 & 2022 & 8.70 & 19 & 29.06 & 155 & 26.97 & 6.63 & 15 & 3 & .09 & .4 & 1.5 & \text { KAO } & 1.6 \mathrm{X} & 78\end{array}$

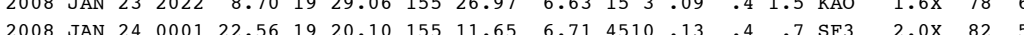

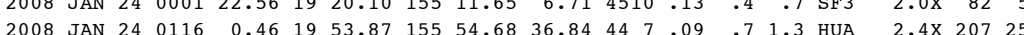

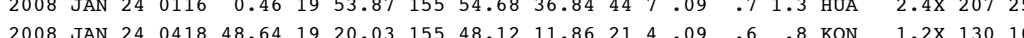

$\begin{array}{llllllllllllllllll}2008 & \text { JAN } 24 & 1710 & 38.93 & 19 & 2.18 & 156 & 18.05 & 42.47 & 37 & 8 & .10 & 1.1 & 1.9 & \text { KON } & 2.2 \times & 289 & 52\end{array}$

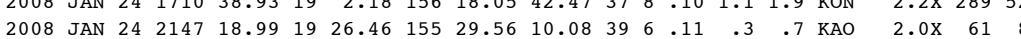

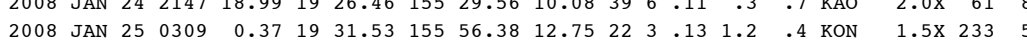
$\begin{array}{lllllllllllllllll}2008 \text { JAN } 25 & 0446 & 11.38 & 20 & 42.34 & 155 & 51.91 & 34.04 & 18 & 2 & .17 & 3.0 & 2.1 & \text { DIS } & 2.1 \times & 327 & 65\end{array}$

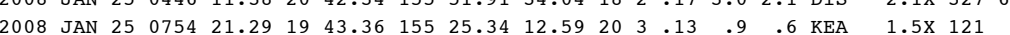

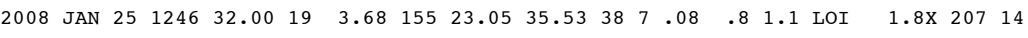

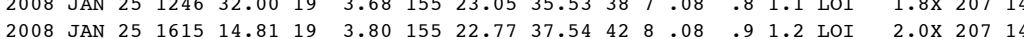

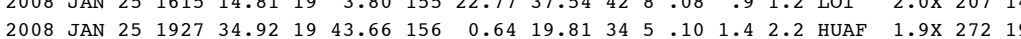

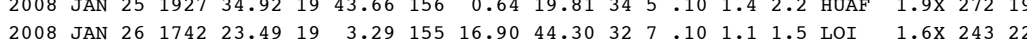

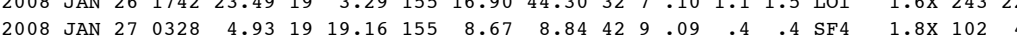

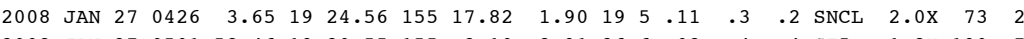

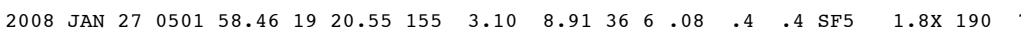

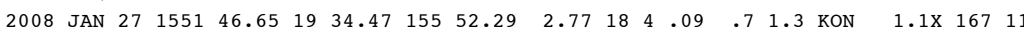

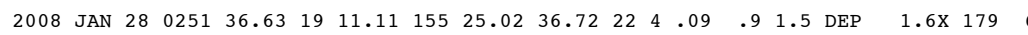

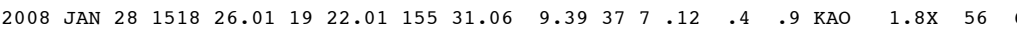

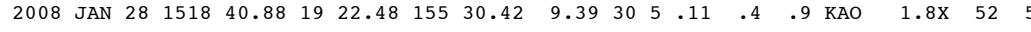

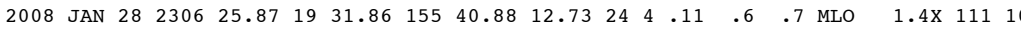

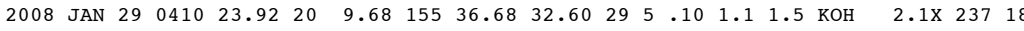

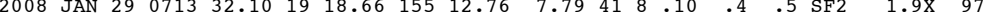

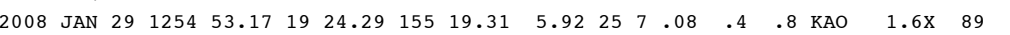

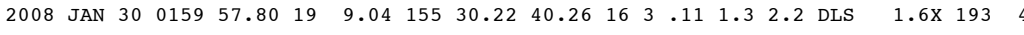

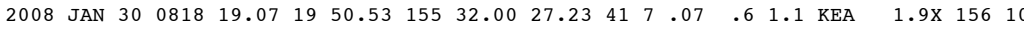

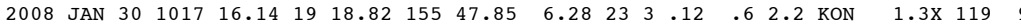

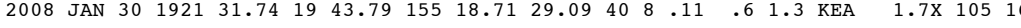

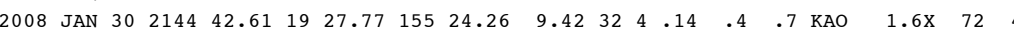

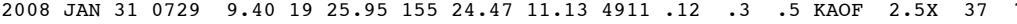

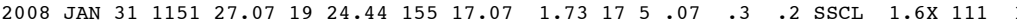

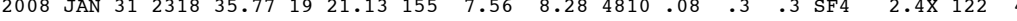

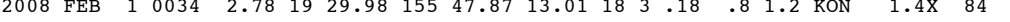
$\begin{array}{lllllllllllllllllll}2008 & \text { FEB } & 1 & 0657 & 18.79 & 19 & 11.36 & 155 & 29.99 & 0.01 & 38 & 8 & .12 & .4 & .3 & \text { LSW } & \# & 1.7 \mathrm{X} & 87\end{array}$

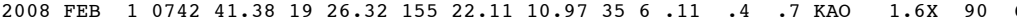
$\begin{array}{lllllllllllllllllll}2008 & \text { FEB } & 1 & 1118 & 14.13 & 19 & 52.17 & 156 & 6.39 & 43.70 & 19 & 3 & .09 & 1.6 & 2.5 & \text { HUA } & 1.9 \mathrm{X} & 289 & 35\end{array}$

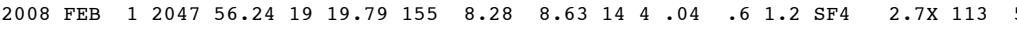

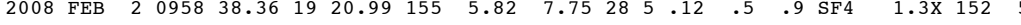

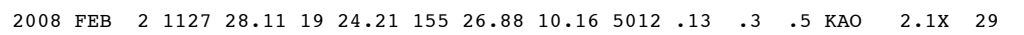


---ORIGIN TIME (HST)-- -LAT N-- --LON W-- DEPTH N N RMS ERH ERZ LOC PREF AZ MIN YEAR MON DA HRMN SEC DEG MIN DEG MIN KM RD S SEC KM KM REMKS MAG GAP DS $\begin{array}{llllllllllllllllllll}2008 & \text { FEB } & 2 & 1128 & 0.14 & 19 & 24.30 & 155 & 26 & 56 & 9.39 & 34 & 5 & .10 & .4 & 8 & \text { KAO } & 1.8 X & 53 & 4\end{array}$

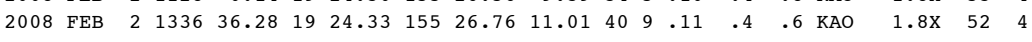
$\begin{array}{lllllllllllllllllll}2008 & \text { FEB } & 2 & 2332 & 45.27 & 19 & 29.51 & 154 & 48.73 & 14.10 & 22 & 5 & .08 & 1.3 & .4 & \text { LER } & 1.7 \times & 281 & 12\end{array}$ $\begin{array}{llllllllllllllll}2008 & & & \end{array}$

$\begin{array}{lllllllllllllllllll}2008 & \text { FEB } & 5 & 0233 & 57.40 & 18 & 57.06 & 155 & 12.01 & 44.67 & 39 & 5 & .09 & 1.4 & 1.5 & \text { LOI } & 1.9 \times & 248 & 36\end{array}$

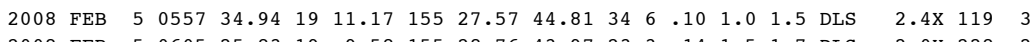

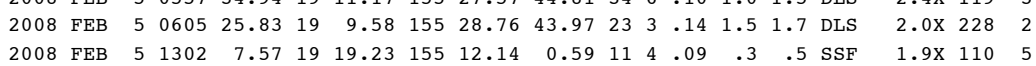

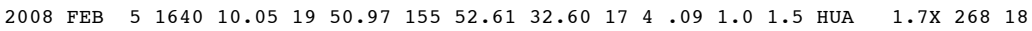

$\begin{array}{lllllllllllllllllll}2008 & \text { FEB } & 6 & 0052 & 28.80 & 19 & 19.96 & 155 & 12.02 & 7.61 & 45 & 9 & .13 & .4 & .6 & \text { SF3 } & 1.8 \mathrm{X} & 82 & 5\end{array}$ $\begin{array}{llllllllllllllllll}2008 & \text { FEB } & 6 & 0113 & 50.55 & 19 & 27.15 & 155 & 16.50 & 26.05 & 5111 & .12 & .5 & .8 & \text { DEPF } & 3.2 \mathrm{x} & 51 & 4\end{array}$ $\begin{array}{llllllllllllllllll}2008 & \text { FEB } & 6 & 0354 & 12.14 & 19 & 6.99 & 155 & 49.45 & 41.26 & 5011 & .09 & .7 & 1.1 & \text { KON } & 2.5 \times & 212 & 7 \\ 2008 & \text { FFB } & 6 & 1113 & 2.92 & 19 & 11.60 & 155 & 28.22 & 10.62 & 43 & 9.15 & .4 & .7 & \text { SW } & 2.6 \times & 111 & 4\end{array}$

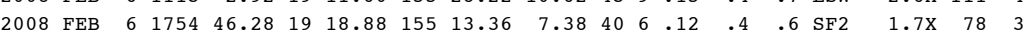

$\begin{array}{lllllllllllllllllll}2008 & \text { FEB } & 6 & 1848 & 4.16 & 19 & 27.08 & 155 & 16.17 & 26.79 & 45 & 7 & .11 & .5 & .8 & \text { DEP } & 2.1 \mathrm{X} & 51 & 4\end{array}$

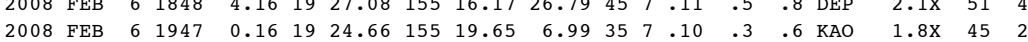

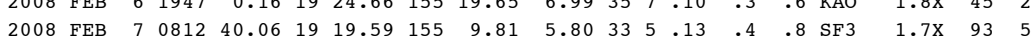

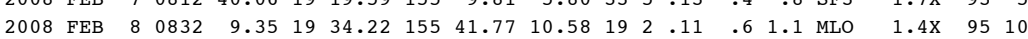

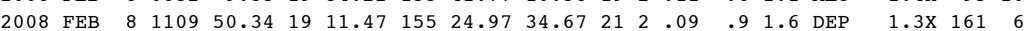

$\begin{array}{lllllllllllllllllll}2008 & \text { FEB } & 8 & 1635 & 24.63 & 19 & 19.84 & 155 & 12.05 & 7.32 & 37 & 7 & .11 & .4 & .6 & \mathrm{SF} 3 & 1.9 \mathrm{X} & 84 & 5\end{array}$

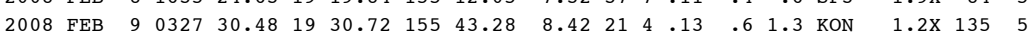

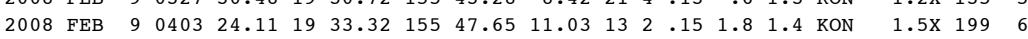

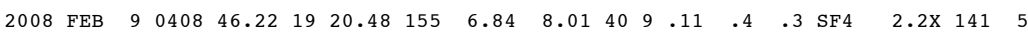

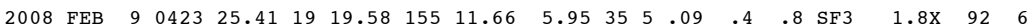

$\begin{array}{lllllllllllllllllll}2008 & \text { FEB } & 9 & 1007 & 1.16 & 19 & 25.29 & 155 & 30.53 & 9.72 & 38 & 6 & .10 & .3 & .7 & \text { KAO } & 1.8 \mathrm{X} & 69 & 7\end{array}$

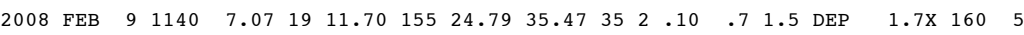
$\begin{array}{llllllllllllllllllll}2008 & \text { FEB } & 9 & 1157 & 40.28 & 19 & 28.60 & 155 & 51.39 & 9.84 & 31 & 4 & .21 & .7 & .7 & \text { KON } & 1.7 \mathrm{X} & 98 & 7\end{array}$ $\begin{array}{lllllllllllllllllll}2008 & \text { FEB } & 9 & 1302 & 26.18 & 19 & 15.74 & 156 & 14.72 & 32.96 & 31 & 5 & .10 & 1.5 & 2.4 & \text { KON } & 2.1 \times & 304 & 39\end{array}$

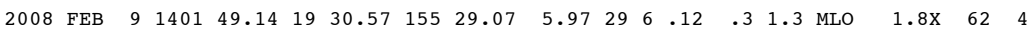

$\begin{array}{lllllllllllllllllll}2008 & \text { FEB } & 9 & 1538 & 17.61 & 19 & 51.52 & 155 & 40.81 & 14.51 & 17 & 2 & .09 & 1.2 & .6 & \text { KEA } & 1.5 \times & 222 & 2\end{array}$

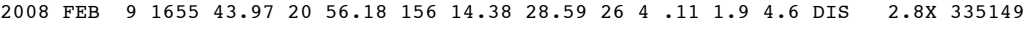
$\begin{array}{lllllllllllllllllll}2008 & \text { FEB } & 9 & 1754 & 30.38 & 19 & 21.40 & 155 & 18.52 & 1.91 & 22 & 5 & .09 & .3 & .5 & \text { SWR } & 1.8 \mathrm{X} & 52 & 5\end{array}$

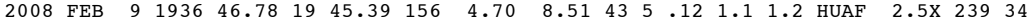

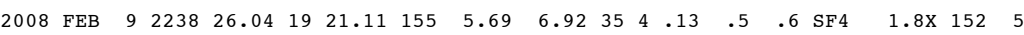

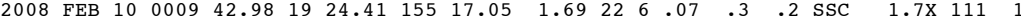

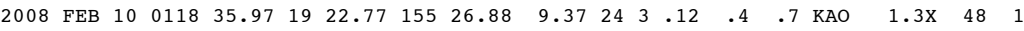

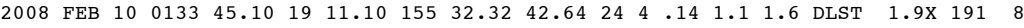

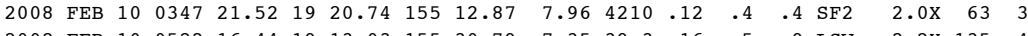

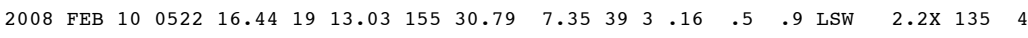

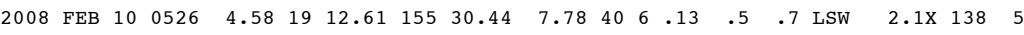

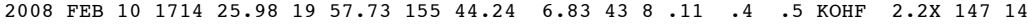

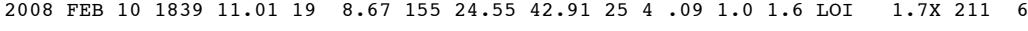

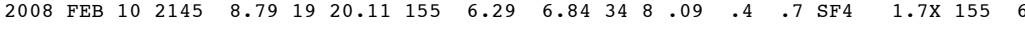
$\begin{array}{lllllllllllllll}2008 & \text { FEB } & 11 & 1307 & 42.48 & 19 & 25.45 & 155 & 19.42 & 4.99 & 25 & 7 & .11 & .4 & .9 \\ \text { KAO } & 1.6 \times & 131 & 3\end{array}$
---ORIGIN TTME (HST)-- -LAT N-- --LON W-- DEPTH N N RMS ERH ERZ LOC PREF AZ MIN YEAR MON DA HRMN SEC DEG MIN DEG MIN KM RD S SEC KM KM REMKS MAG GAP DS

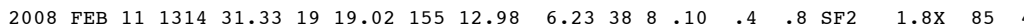

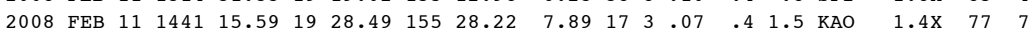

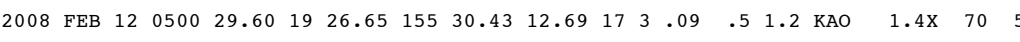

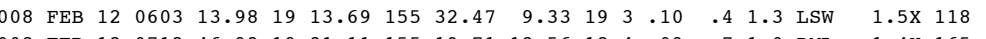

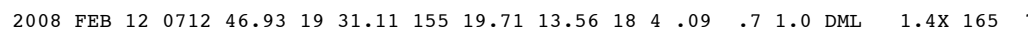

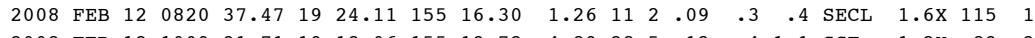

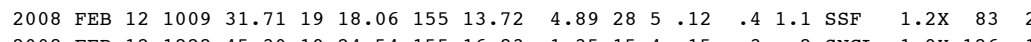

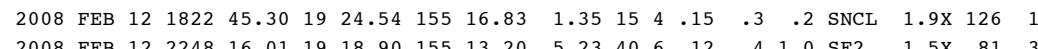

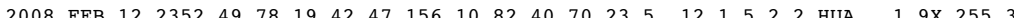

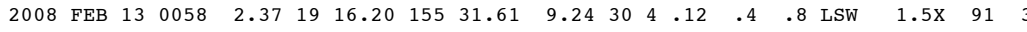

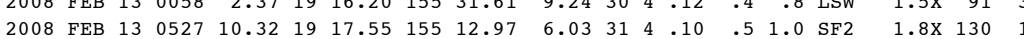

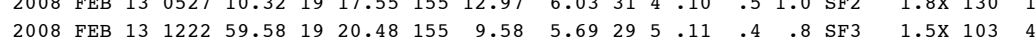

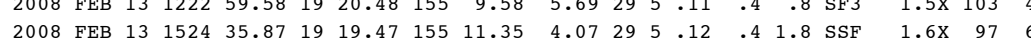

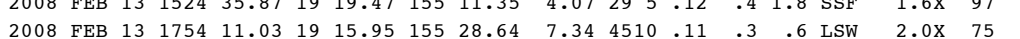

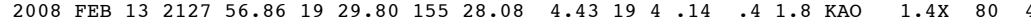

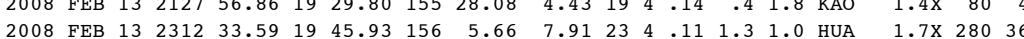

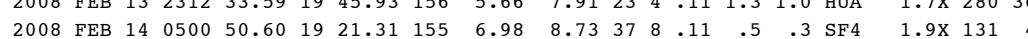

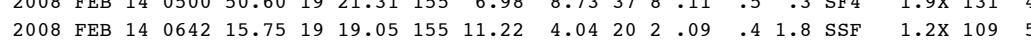

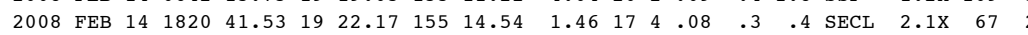

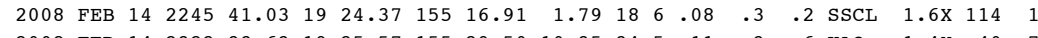

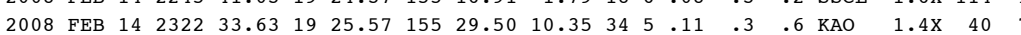

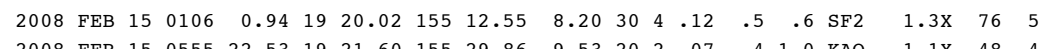

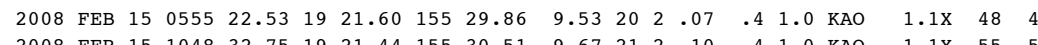

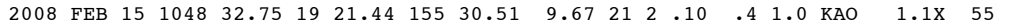

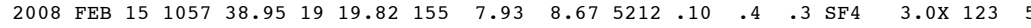

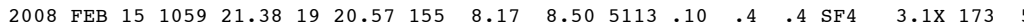

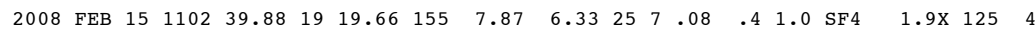

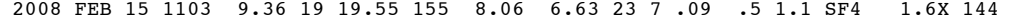

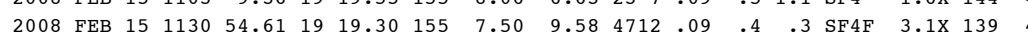

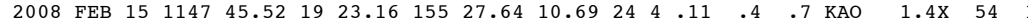

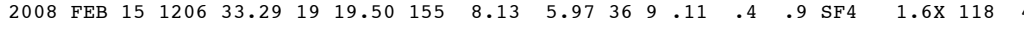

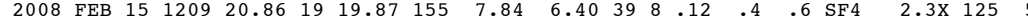

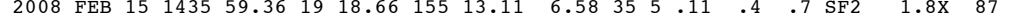

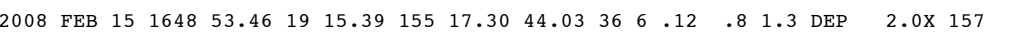

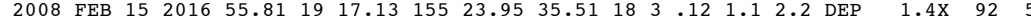

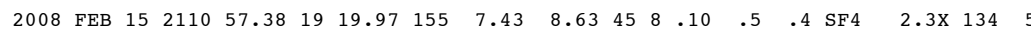

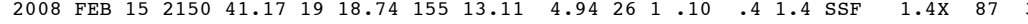

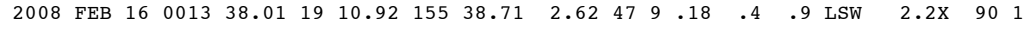

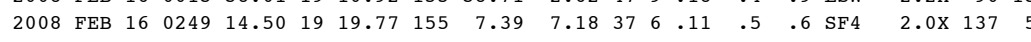

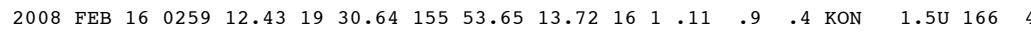

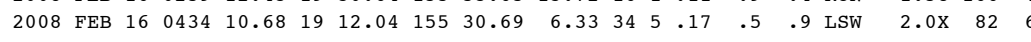

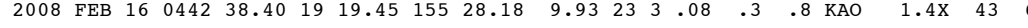

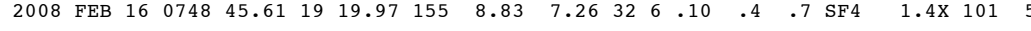

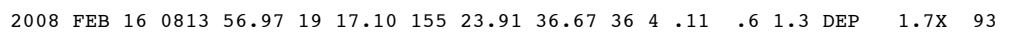


---ORIGIN TIME (HST)-- -LAT N-- --LON W-- DEPTH N N RMS ERH ERZ LOC PREF AZ MIN YEAR MON DA HRMN SEC DEG MIN DEG MIN KM RD S SEC KM KM REMKS MAG GAP DS

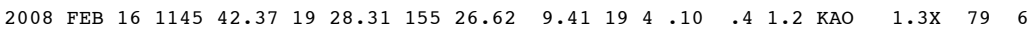

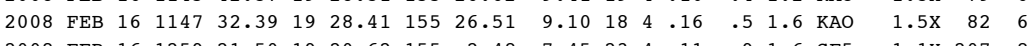
$\begin{array}{lllllllllll}2008 & & \end{array}$

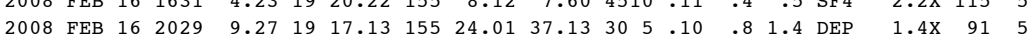

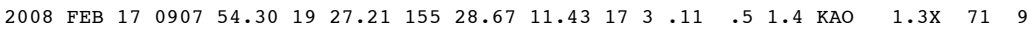

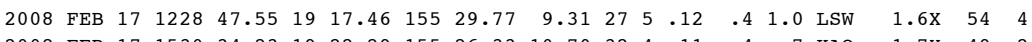

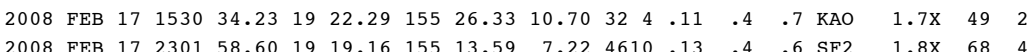

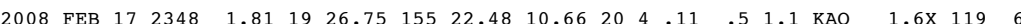

$\begin{array}{llllllllllllllllll}2008 & \text { FEB } 18 & 0618 & 32.21 & 19 & 15.88 & 155 & 29.59 & 10.79 & 43 & 9 & .12 & .3 & .6 & \text { LSW } & 2.2 \mathrm{X} & 59 & 2\end{array}$

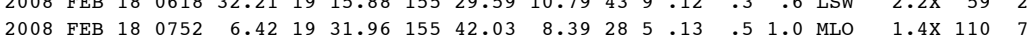

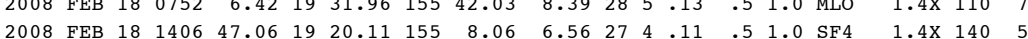

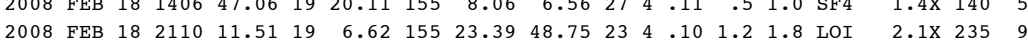

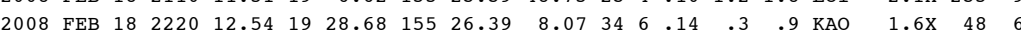

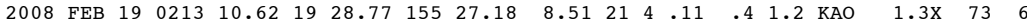

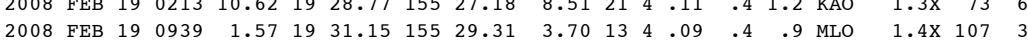

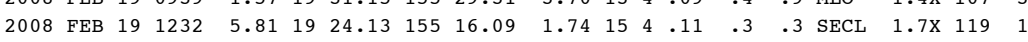

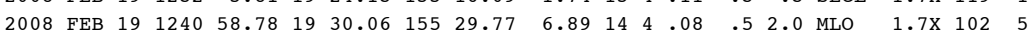

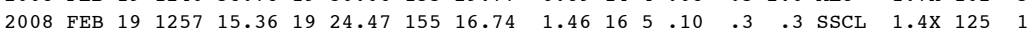

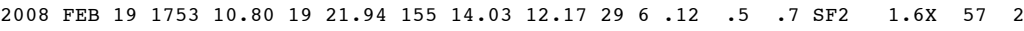

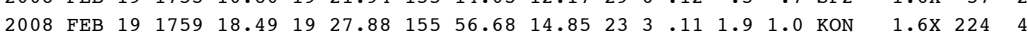

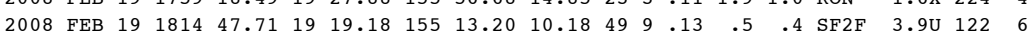

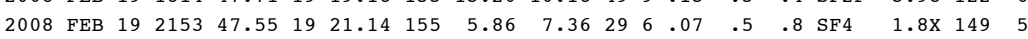

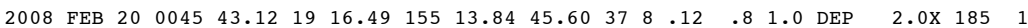

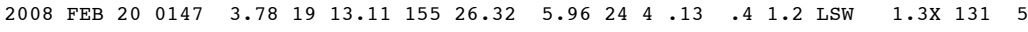

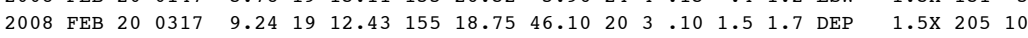

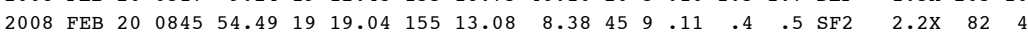

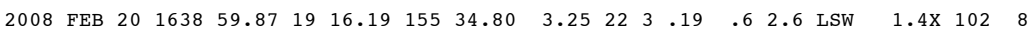

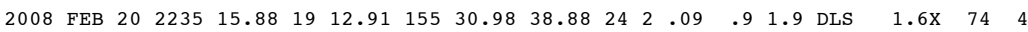

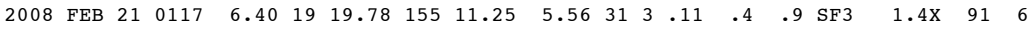

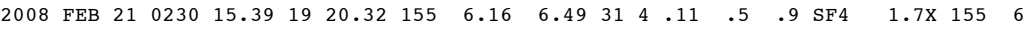

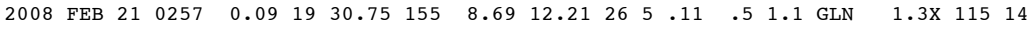

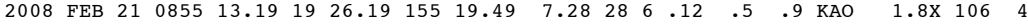

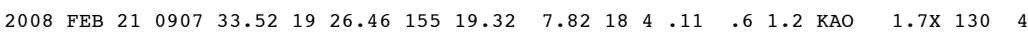

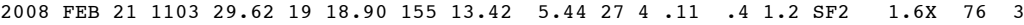

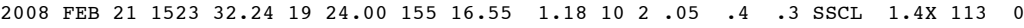

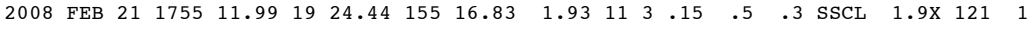

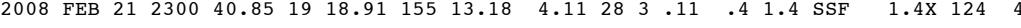

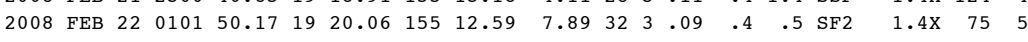

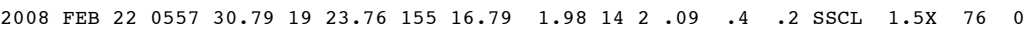

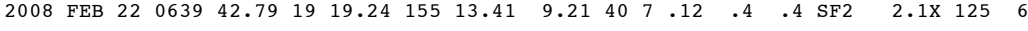

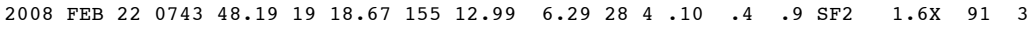

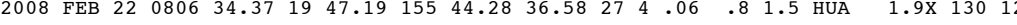

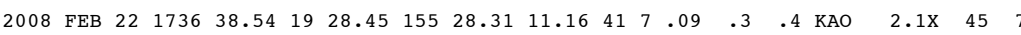

---ORIGIN TIME (HST)-- -LAT N-- --LON W-- DEPTH N N RMS ERH ERZ LOC PREF AZ MIN YEAR MON DA HRMN SEC DEG MIN DEG MIN KM RD S SEC KM KM REMKS MAG GAP DS

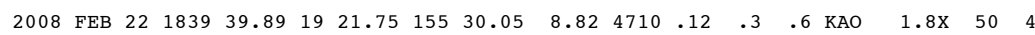

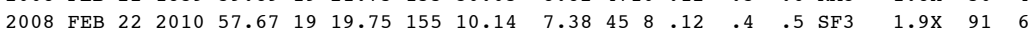

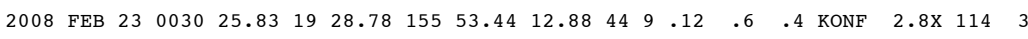

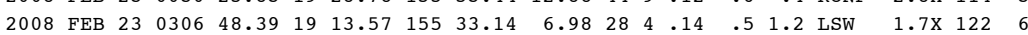

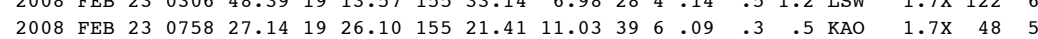

$\begin{array}{lllllllllllllllll}2008 & \text { FEB } & 23 & 0843 & 33.02 & 19 & 19.87 & 155 & 6.45 & 6.50 & 22 & 1.12 & .6 & 1.3 & \text { SF } 4 & 1.3 \times & 156\end{array}$

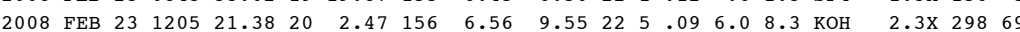

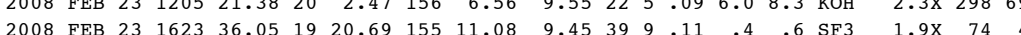

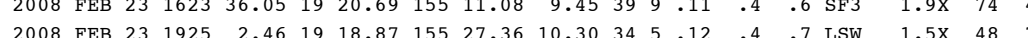

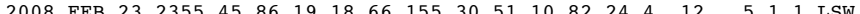

$\begin{array}{llllllllllllllllll}2008 & \text { FEB } 24 & 0019 & 50.72 & 19 & 20.42 & 155 & 12.53 & 7.01 & 28 & 5 & .11 & .5 & .8 & \mathrm{SF} 2 & 1.3 \mathrm{X} & 70 & 4\end{array}$

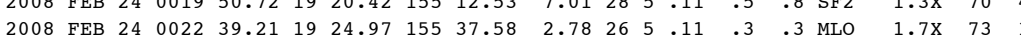

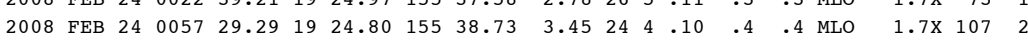

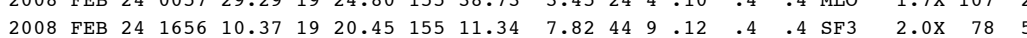

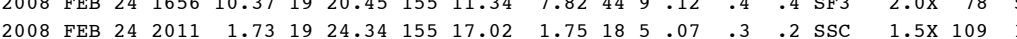

$\begin{array}{llllllllllllllllll}2008 & \text { FEB } 24 & 2208 & 20.71 & 19 & 19.09 & 155 & 13.53 & 6.73 & 32 & 3 & .12 & .5 & .8 & \text { SF2 } & 1.7 \times & 112 & 4\end{array}$

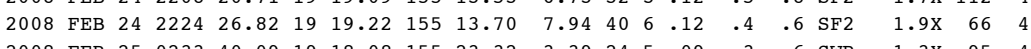

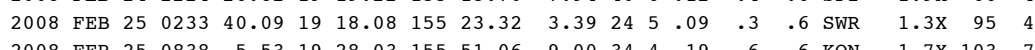

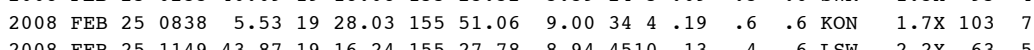

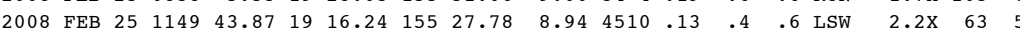

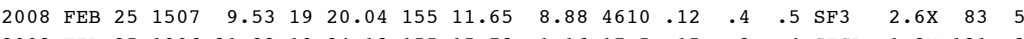

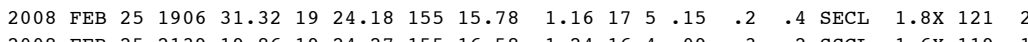

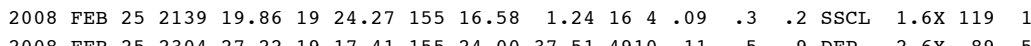

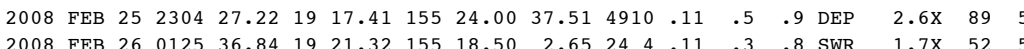

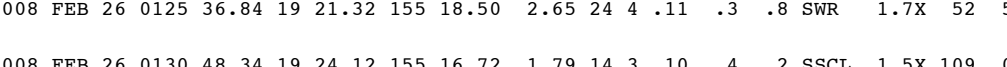

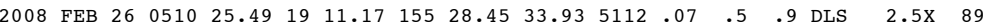

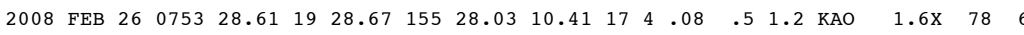

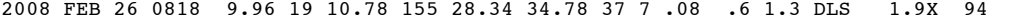
$\begin{array}{llllllllllll}2008 & \text { FEB } & 26 & 0824 & 52.38 & 19 & 24.37 & 155 & 16.24 & 1.76 & 11 & 3 \\ .08 & .3 & .5 & \text { SECL } & 1.2 \times & 131\end{array}$

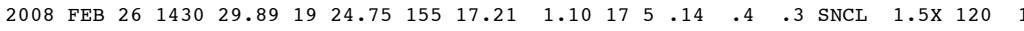

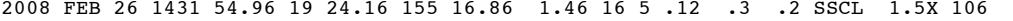

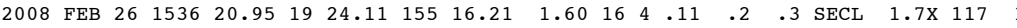

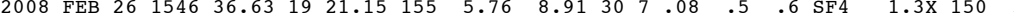

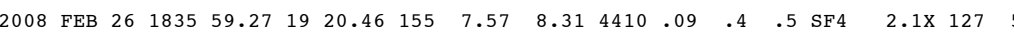

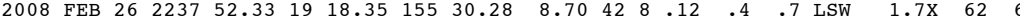
$\begin{array}{llllllllllllllllll}2008 & \text { FEB } 27 & 0631 & 28.74 & 19 & 26.70 & 155 & 30.46 & 11.88 & 23 & 5 & .09 & .4 & 1.3 & \text { KAO } & 1.2 \mathrm{x} & 70 & 9\end{array}$

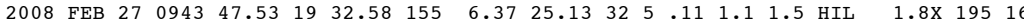

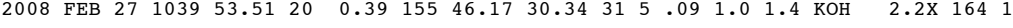

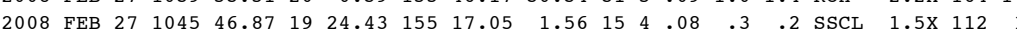

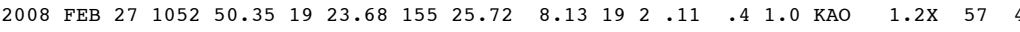

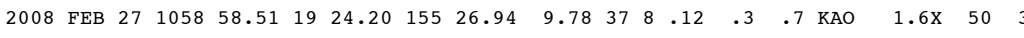

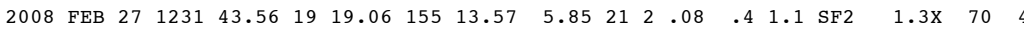

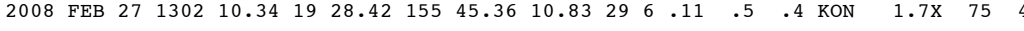

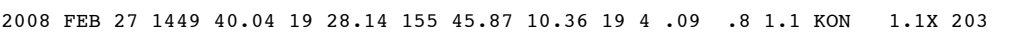


---ORIGIN TIME (HST)-- -LAT N-- --LON W-- DEPTH N N RMS ERH ERZ LOC PREF AZ MIN YEAR MON DA HRMN SEC DEG MIN DEG MIN KM RD S SEC KM KM REMKS MAG GAP DS

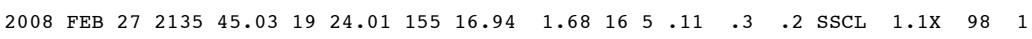

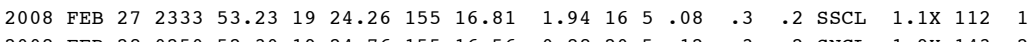

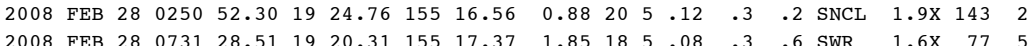

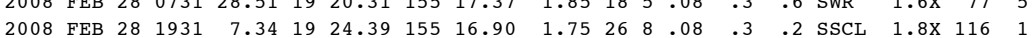

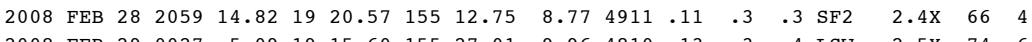

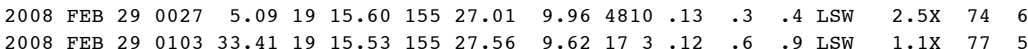

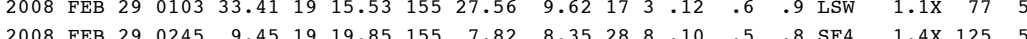
$\begin{array}{llllllllllllll}2008 & & \end{array}$

$\begin{array}{llllllllllllllllll}2008 & \text { FEB } 29 & 0332 & 29.44 & 19 & 29.93 & 155 & 29.65 & 7.02 & 15 & 4 & .08 & .4 & 2.0 & \text { KAO } & 1.5 \times & 100 & 5\end{array}$

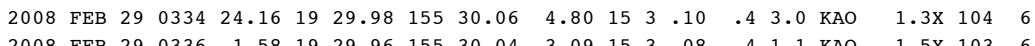

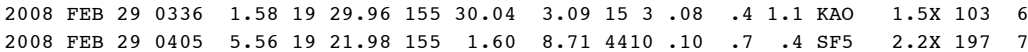

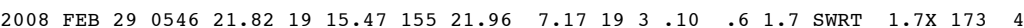

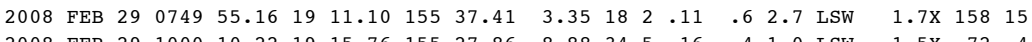

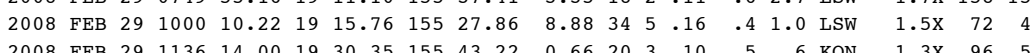

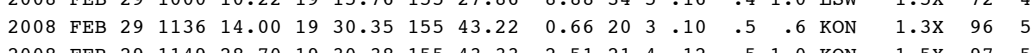

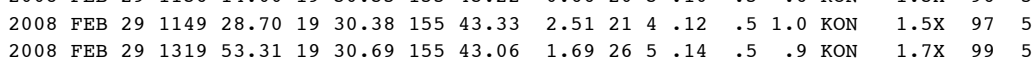

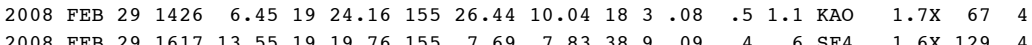

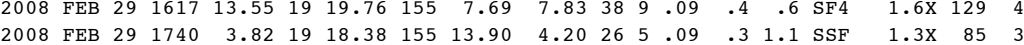

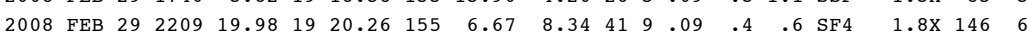

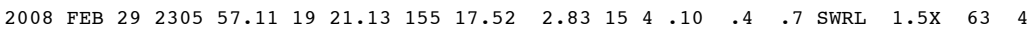

$\begin{array}{lllllllllllllllllll}2008 & \text { MAR } & 1 & 0114 & 37.67 & 19 & 30.01 & 155 & 28.53 & 4.81 & 19 & 4 & .08 & .4 & 1.6 & \text { MLO } & 1.3 \mathrm{X} & 90 & 4\end{array}$

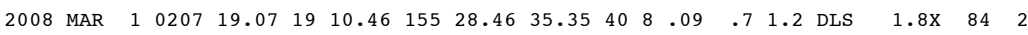

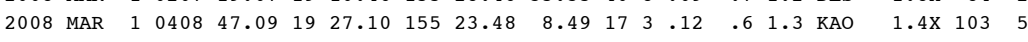
$\begin{array}{lllllllllllllllllll}2008 & \text { MAR } & 1 & 0455 & 20.94 & 19 & 13.01 & 155 & 32.47 & 6.71 & 21 & 3 & .13 & .5 & 1.5 & \text { LSW } & 1.4 \times & 126 & 6\end{array}$

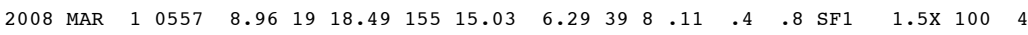

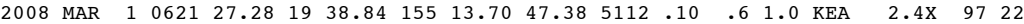

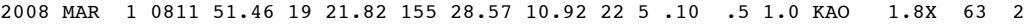

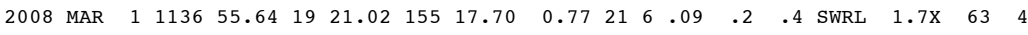

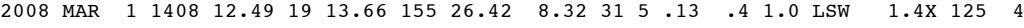

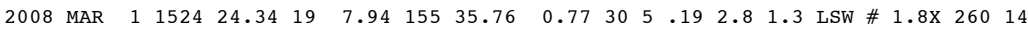

$\begin{array}{lllllllllllllllllll}2008 & \text { MAR } & 1 & 2004 & 57.32 & 19 & 19.83 & 155 & 53.78 & 13.56 & 23 & 4 & .11 & .9 & .4 & \text { KON } & 1.4 \mathrm{X} & 194 & 8\end{array}$

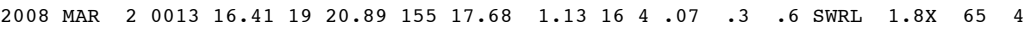
2008 MAR $2 \begin{array}{llllllllllllllllll}0 & 0644 & 34.51 & 19 & 24.12 & 155 & 17.11 & 3.47 & 15 & 4 & .13 & .4 & .3 & \text { SSCL } & 1.6 \mathrm{X} & 96 & 1\end{array}$ 2008 MAR $2 \begin{array}{llllllllllllllllll}0646 & 25.14 & 19 & 24.26 & 155 & 16.49 & 2.45 & 16 & 6 & .10 & .3 & .2 & \text { SECL } & 1.6 \mathrm{X} & 120 & 1\end{array}$

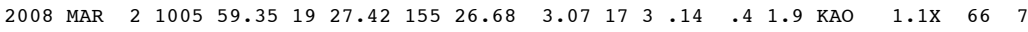

$\begin{array}{lllllllllllllllllll}2008 & \text { MAR } & 2 & 1305 & 36.91 & 19 & 23.41 & 155 & 27.29 & 9.68 & 32 & 4 & .12 & .3 & .8 & \text { KAO } & 1.5 \mathrm{X} & 47 & 2\end{array}$ $\begin{array}{llllllllllllllllll}2008 & \text { MAR } & 2 & 1345 & 55.84 & 19 & 25.16 & 155 & 15.90 & 16.62 & 4610 & .12 & .4 & .5 & \text { DEP } & 2.3 \mathrm{X} & 46 & 3\end{array}$

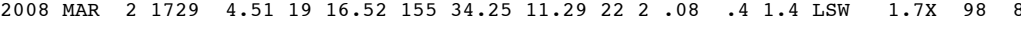
2008 MAR $30 \begin{array}{llllllllllllllll}0 & 28.88 & 19 & 17.38 & 155 & 47.45 & 11.38 & 29 & 3 & .09 & .4 & .5 & \text { KON } & 1.9 \times & 85 & 9\end{array}$

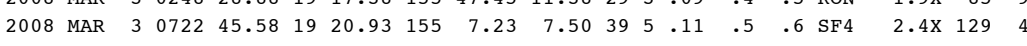

---ORIGIN TIME (HST)-- -LAT N-- --LON W-- DEPTH N N RMS ERH ERZ LOC PREF AZ MIN YEAR MON DA HRMN SEC DEG MIN DEG MIN KM RD S SEC KM KM REMRS MAG GAP DS

2008 MAR $3 \quad \begin{array}{llllllllllllllllll}1425 & 37.88 & 19 & 48.07 & 155 & 20.61 & 34.26 & 13 & 3 & 11 & 1.1 & 1.8 & \text { KEA } & 1.7 \times & 147 & 10\end{array}$

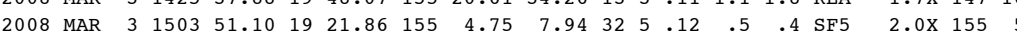
2008 MAR $3 \begin{array}{llllllllllllllllll}19003 & 4.69 & 18 & 58.92 & 155 & 13.68 & 46.27 & 44 & 8 & 11 & 1.1 & 1.3 & \text { LOI } & 2.4 \times & 240 & 32\end{array}$ 2008 MAR $3 \begin{array}{llllllllllllllll}2 & 2119 & 10.06 & 19 & 24.28 & 15 & 17.11 & 1.76 & 16 & 4 & 07 & 2 & 2 & \text { SSCL } & 1.5 \times & 103\end{array}$

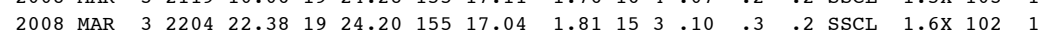

$\begin{array}{lllllllllllllllllll}2008 & \text { MAR } & 4 & 0858 & 35.16 & 19 & 16.03 & 155 & 29.10 & 10.46 & 40 & 5 & .15 & .4 & .7 & \text { LSW } & 2.0 \mathrm{X} & 60 & 3\end{array}$

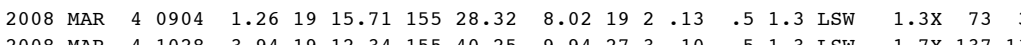

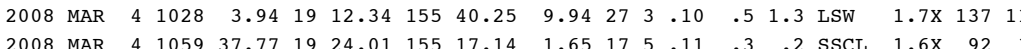

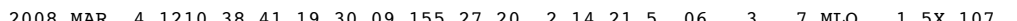

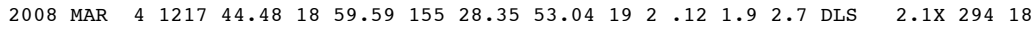
2008 MAR $4 \begin{array}{lllllllllllllll}2209 & 32.44 & 19 & 18.49 & 155 & 13.29 & 6.72 & 42 & 9 & .11 & 4 & 7 & \text { SF } 2 & 1.7 \times & 85\end{array}$

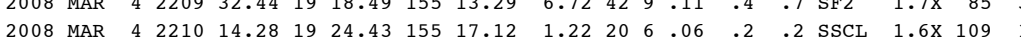

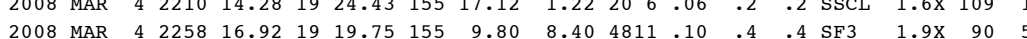

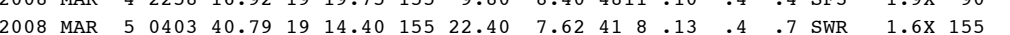

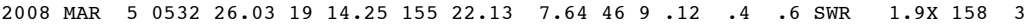

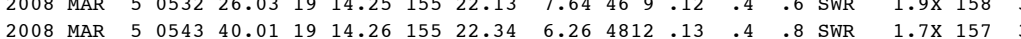

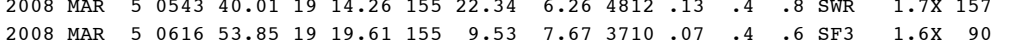

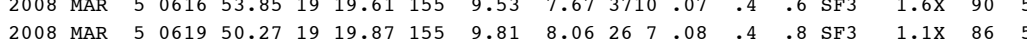

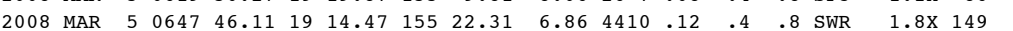

$\begin{array}{lllllllllllllllllll}2008 & \text { MAR } & 5 & 0743 & 48.81 & 19 & 26.40 & 155 & 22.24 & 10.29 & 30 & 6 & .09 & .4 & .8 & \text { KAO } & 1.4 \mathrm{X} & 47 & 7\end{array}$

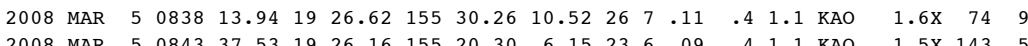

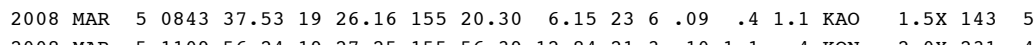

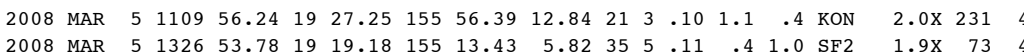

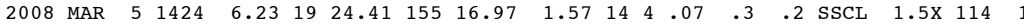

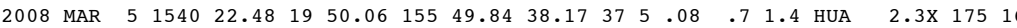

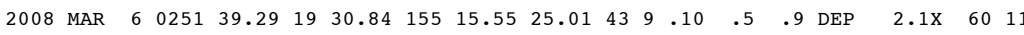
2008 MAR $\quad 6 \quad \begin{array}{llllllllllllllll}0316 & 21.01 & 19 & 19.38 & 155 & 11.85 & 6.07 & 26 & 3 & .09 & .4 & .8 & \text { SF3 } & 1.3 \times & 96\end{array}$

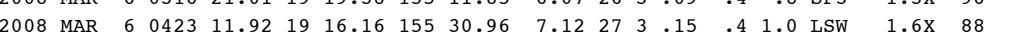

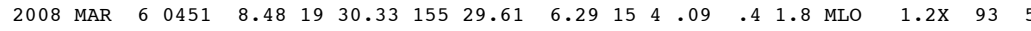

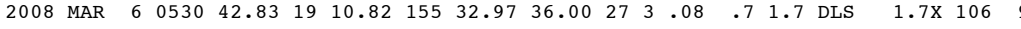

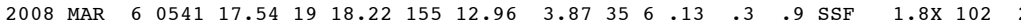

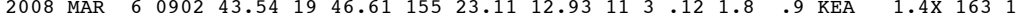
2008 MAR $\quad \begin{array}{lllllllllllllll}1123 & 52.75 & 19 & 18.71 & 155 & 15.37 & 5.17 & 33 & 4 & .11 & .4 & 1.3 & \text { SF } 1 & 1.4 \times & 100\end{array}$

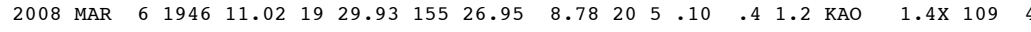

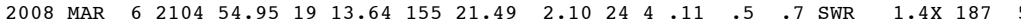

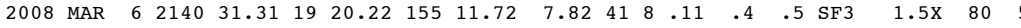

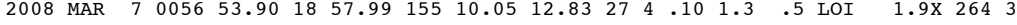

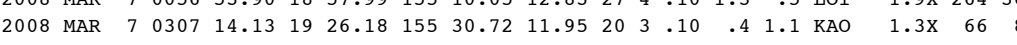

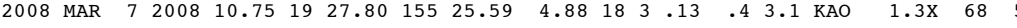

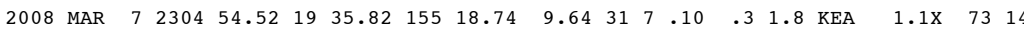

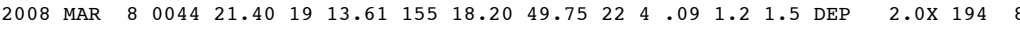

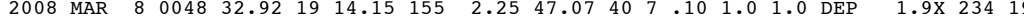

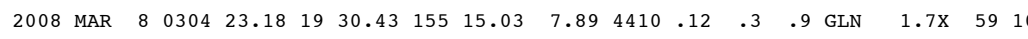


---ORIGIN TIME (HST)-- -LAT N-- --LON W-- DEPTH N N RMS ERH ERZ LOC PREF AZ MIN YEAR MON DA HRMN SEC DEG MIN DEG MIN KM RD S SEC KM KM REMKS MAG GAP DS $\begin{array}{lllllllllllllllllll}2008 & \text { MAR } & 8 & 0508 & 9.57 & 19 & 24.44 & 155 & 16.93 & 1.76 & 29 & 8 & .12 & .3 & .2 & \text { SSCL } & 1.7 \times & 117 & 1\end{array}$

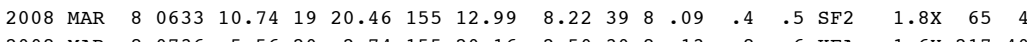

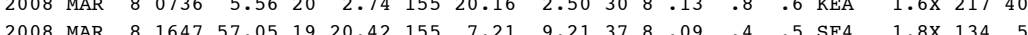

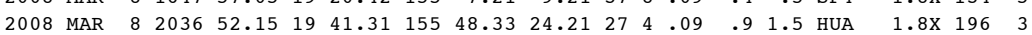

$\begin{array}{lllllllllllllllllll}2008 \text { MAR } & 8 & 2104 & 50.90 & 19 & 10.41 & 155 & 28.58 & 36.34 & 32 & 6 & .09 & .8 & 1.5 & \text { DLS } & 1.6 \mathrm{x} & 82 & 2\end{array}$

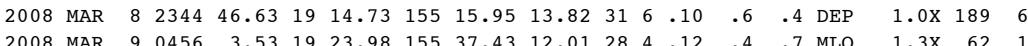

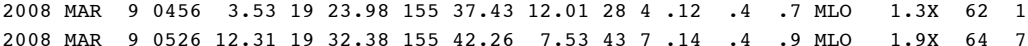

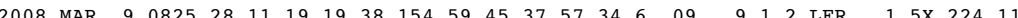

$\begin{array}{llllllllllllllllll}2008 & \text { MAR } 9 & 0853 & 11.21 & 19 & 11.07 & 155 & 28.37 & 34.19 & 28 & 5 & .09 & .6 & 1.5 & \text { DLS } & 1.4 \mathrm{X} & 92 & 3\end{array}$

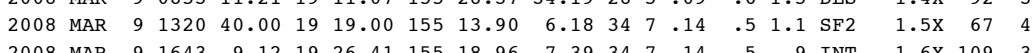

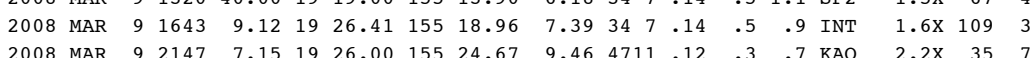

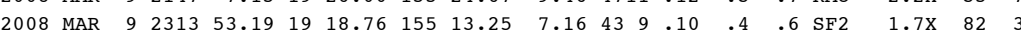

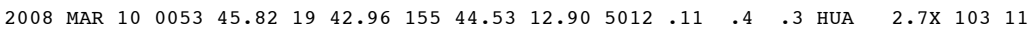

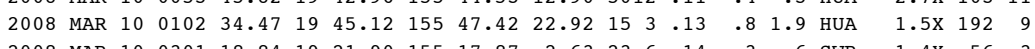

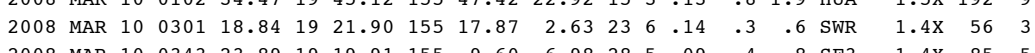

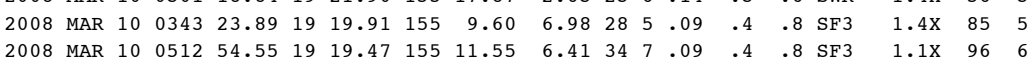

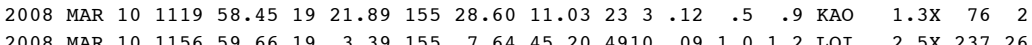

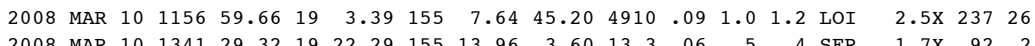

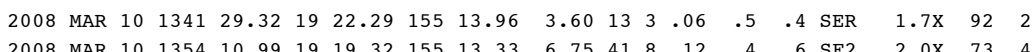

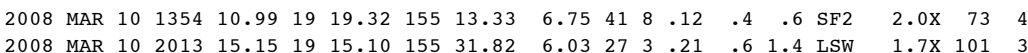

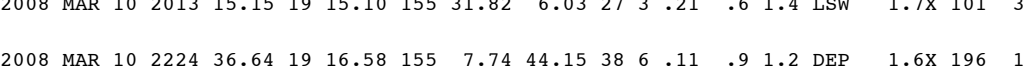

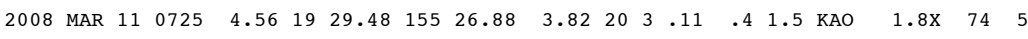

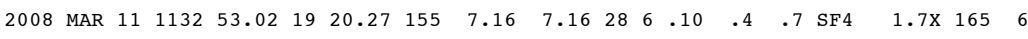

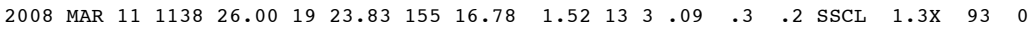

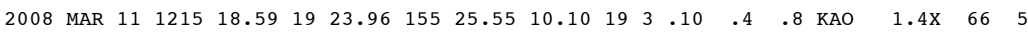

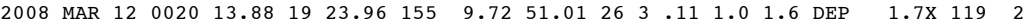

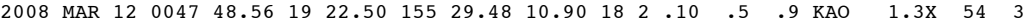

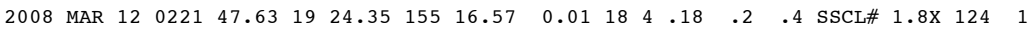

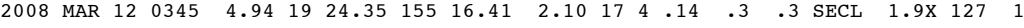

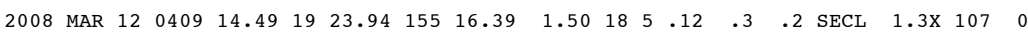

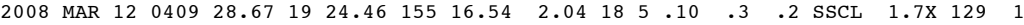

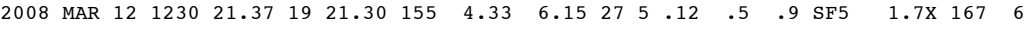

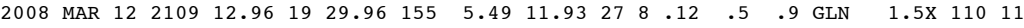

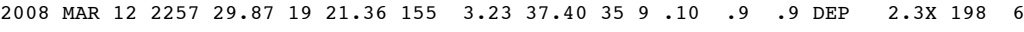

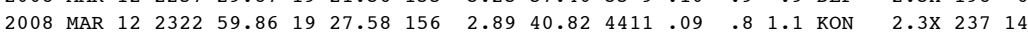

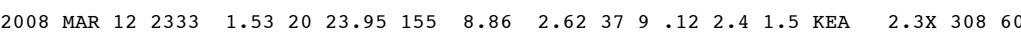

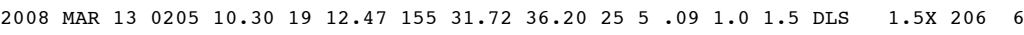

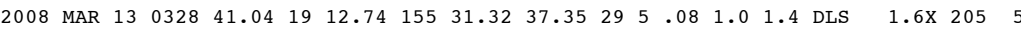

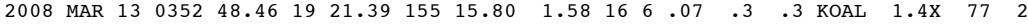
$\begin{array}{lllllllllllllll}2008 & \text { MAR } & 13 & 0353 & 16.86 & 19 & 21.50 & 155 & 15.90 & 1.75 & 15 & 5 & .05 & .3 & .3 \\ \text { KOAL } & 1.3 \times & 85 & 2\end{array}$
---ORIGIN TTME (HST)-- -LAT N-- --LON W-- DEPTH N N RMS ERH ERZ LOC PREF AZ MIN YEAR MON DA HRMN SEC DEG MIN DEG MIN KM RD S SEC KM KM REMKS MAG GAP DS

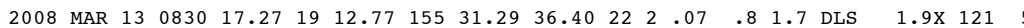

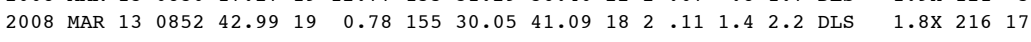

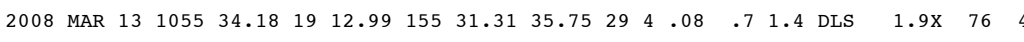

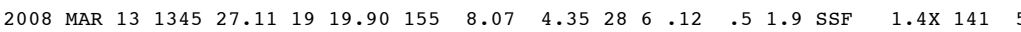

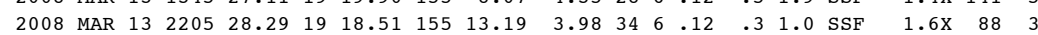

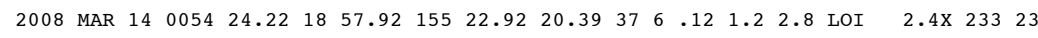

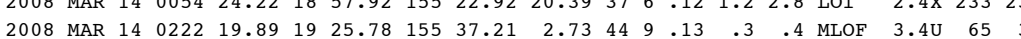

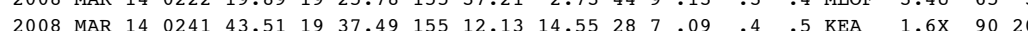

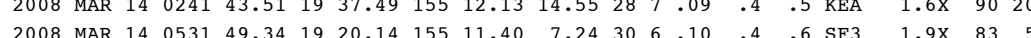

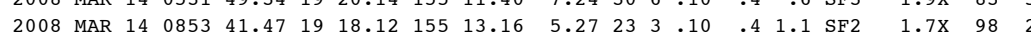

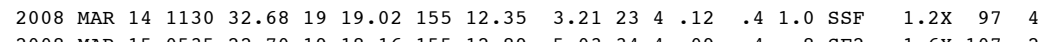

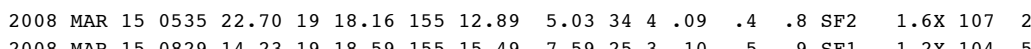

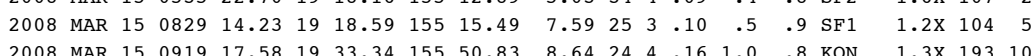

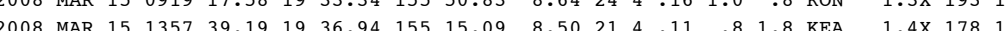

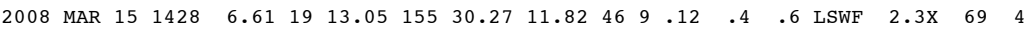

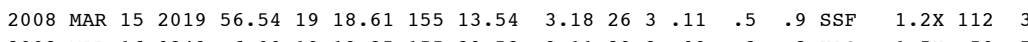

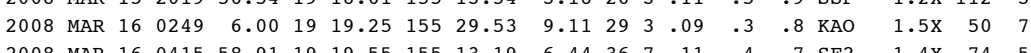

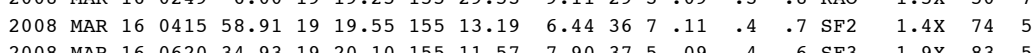

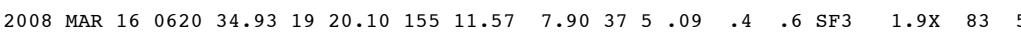

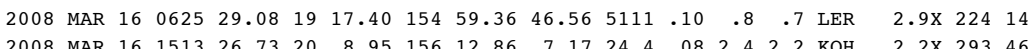

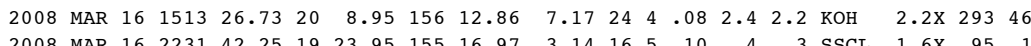

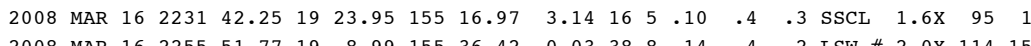

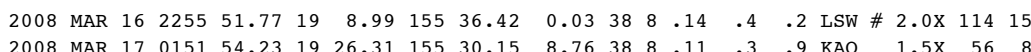
$\begin{array}{llllllllllllllllll}2008 \text { MAR } & 17 & 0151 & 54.23 & 19 & 26.31 & 155 & 30.15 & 8.76 & 38 & 8 & .11 & .3 & .9 & \text { KAO } & 1.5 \times & 56 \\ 8\end{array}$

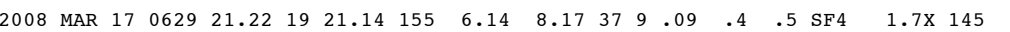

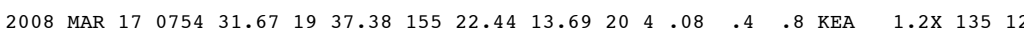

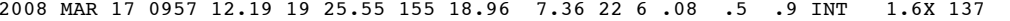

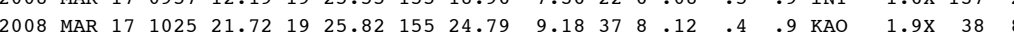

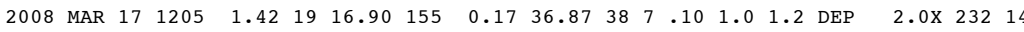

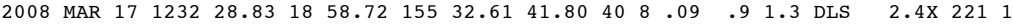
$\begin{array}{llllllllllllllllll}2008 & \text { MAR } & 17 & 1523 & 38.05 & 19 & 19.42 & 155 & 9.97 & 6.61 & 37 & 8 & .10 & .4 & .8 & \mathrm{SF} 3 & 1.8 \mathrm{X} & 98\end{array}$

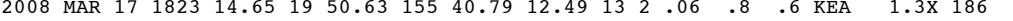

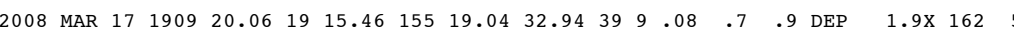

$\begin{array}{llllllllllllllll}2008 & \text { MAR } 17 & 2108 & 42.49 & 19 & 29.99 & 155 & 23.65 & 14.06 & 23 & 6 & .09 & .5 & .6 & \text { DML } & 1.6 \times 112\end{array}$

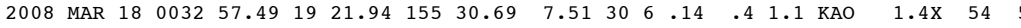

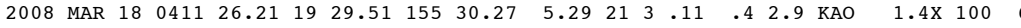

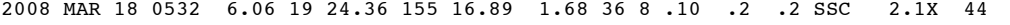

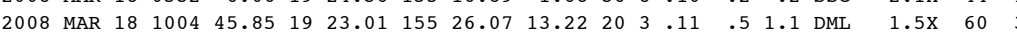

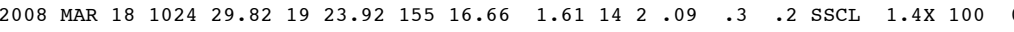

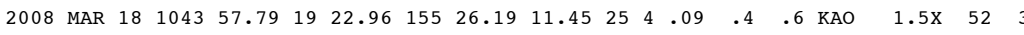

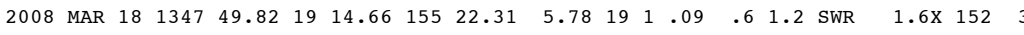

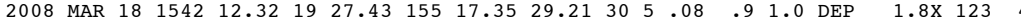

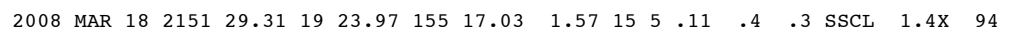


--ORIGIN TIME (HST)-- -LAT N-- --LON W-- DEPTH N N RMS ERH ERZ LOC PREF AZ MIN YEAR MON DA HRMN SEC DEG MIN DEG MIN KM RD S SEC KM KM REMKS MAG GAP DS

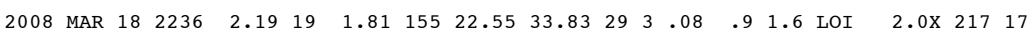

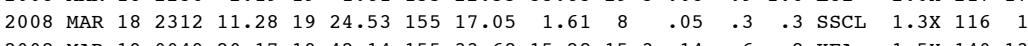

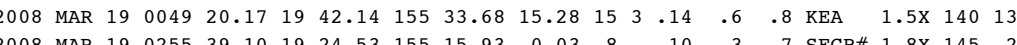

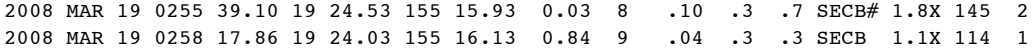

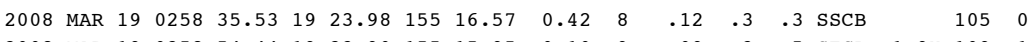

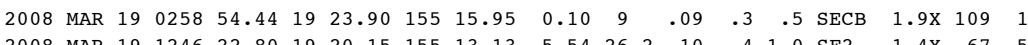

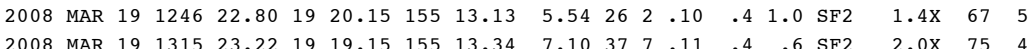

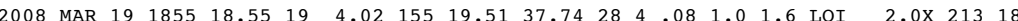

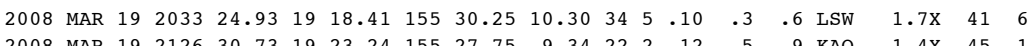

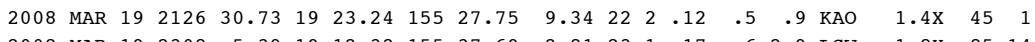

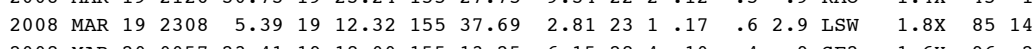

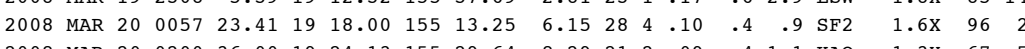

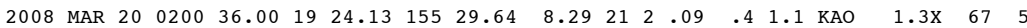

$\begin{array}{llllllllllllllllll}2008 & \text { MAR } 20 & 0452 & 34.26 & 19 & 22.85 & 155 & 30.21 & 8.02 & 21 & 1 & .11 & .4 & .9 & \text { KAO } & 1.6 \mathrm{X} & 49 & 5\end{array}$

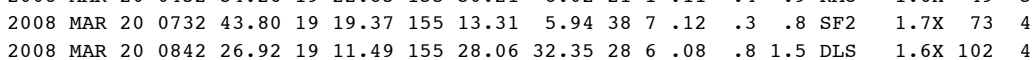

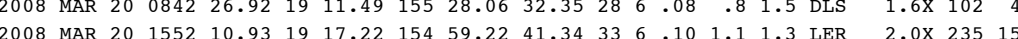

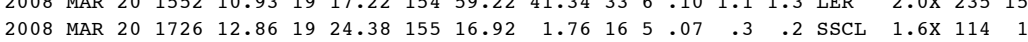

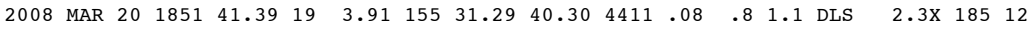

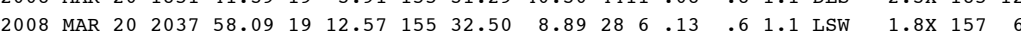

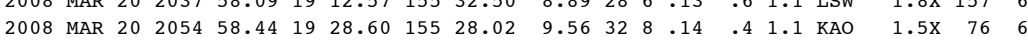

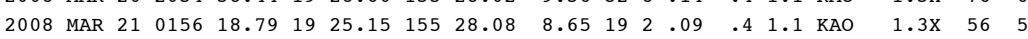

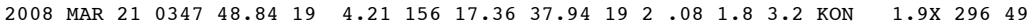

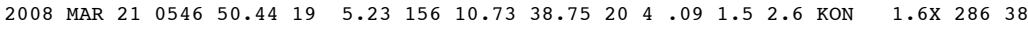

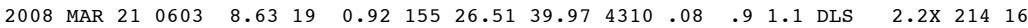

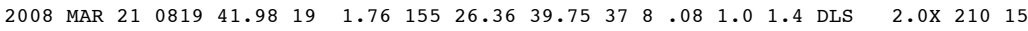

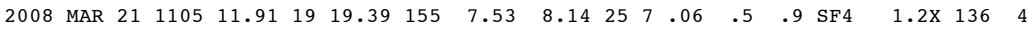

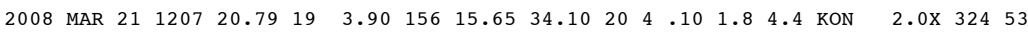

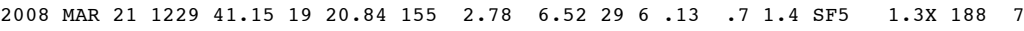

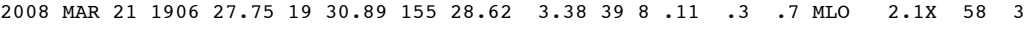

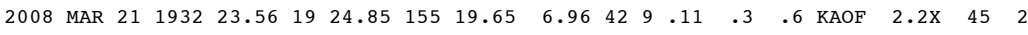

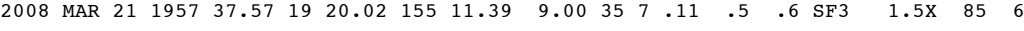

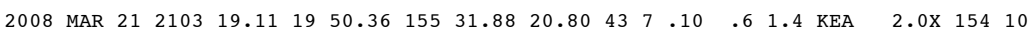

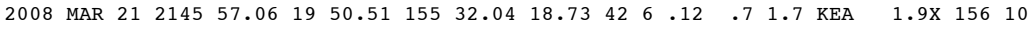

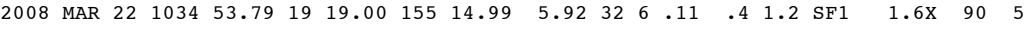

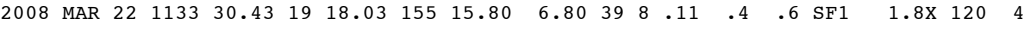

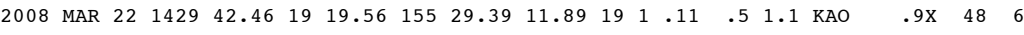

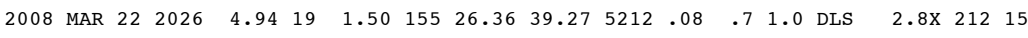

$\begin{array}{llllllllllllllllll}2008 & \text { MAR } 22 & 2225 & 27.47 & 19 & 23.93 & 155 & 16.84 & 1.63 & 14 & 2 & .11 & .3 & .2 & \text { SSCL } & 1.5 \mathrm{X} & 98 & 0\end{array}$

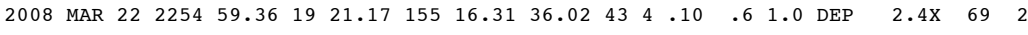

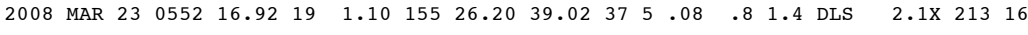

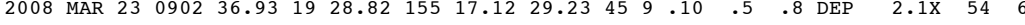

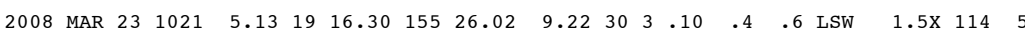

--ORIGIN TIME (HST)-- -LAT N-- --LON W-- DEPTH N N RMS ERH ERZ LOC PREF AZ MIN YEAR MON DA HRMN SEC DEG MIN DEG MIN KM RD S SEC KM KM REMKS MAG GAP DS

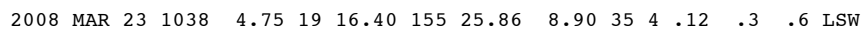

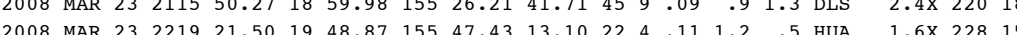

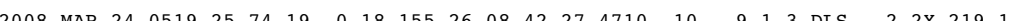
$\begin{array}{lllllllll} & 0.38 & & \end{array}$

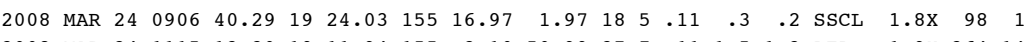

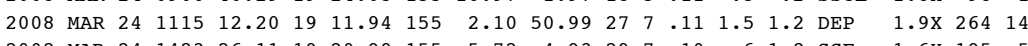

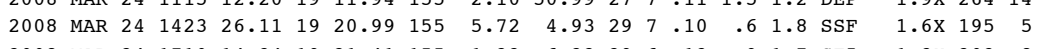

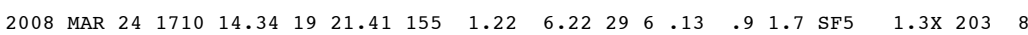

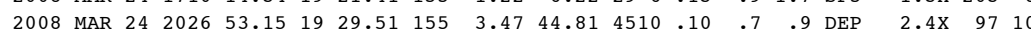

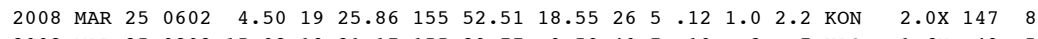
$\begin{array}{lllllllllllllllll}2008 \text { MAR } 25 & 0803 & 15.08 & 19 & 21.17 & 155 & 29.77 & 9.59 & 40 & 7 & .10 & .3 & .7 & \text { KAO } & 1.6 \mathrm{X} & 49 & 5\end{array}$

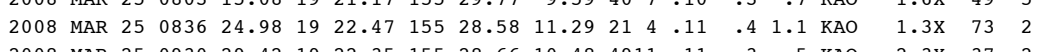

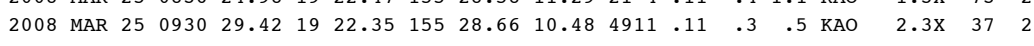

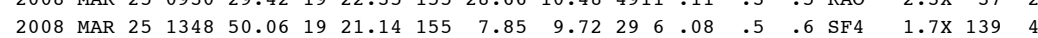

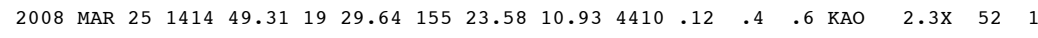

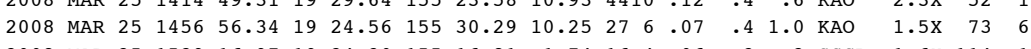

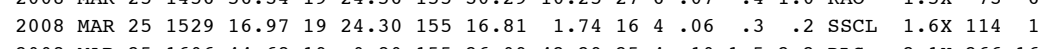

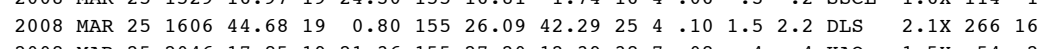

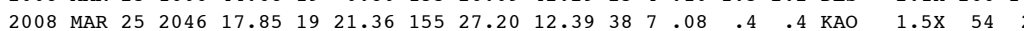

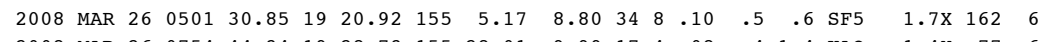

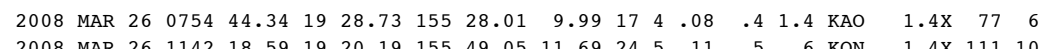

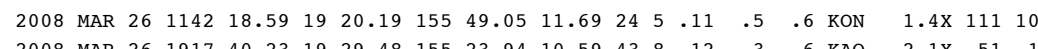

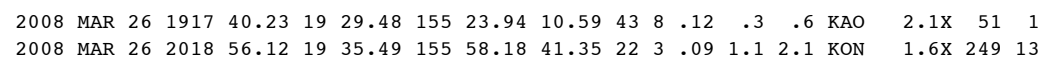

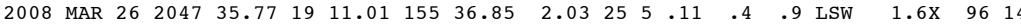

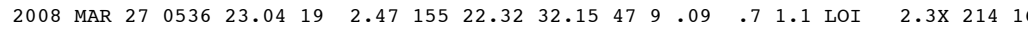

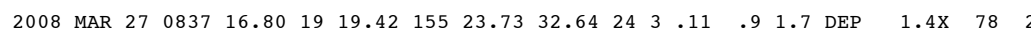

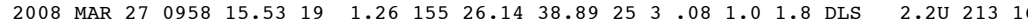

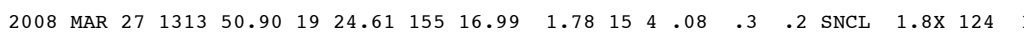

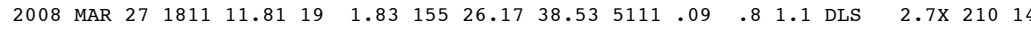

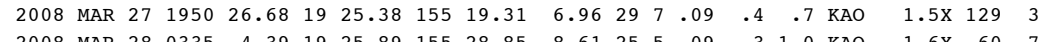

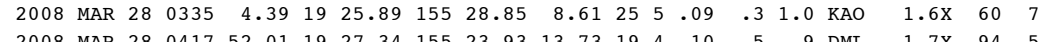

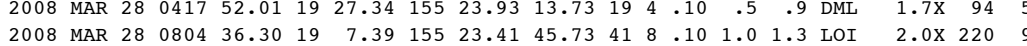

$\begin{array}{lllllllllllllllll}2008 & \text { MAR } 28 & 1042 & 39.96 & 19 & 21.94 & 155 & 4.71 & 10.45 & 32 & 8 & .09 & .6 & .4 & \text { SF5 } & 1.5 \times & 154\end{array}$

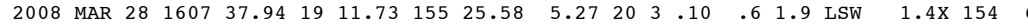

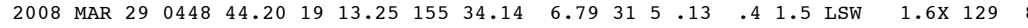

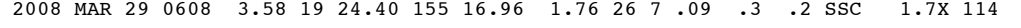

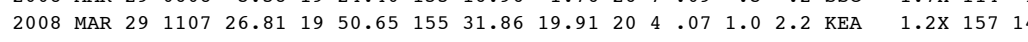

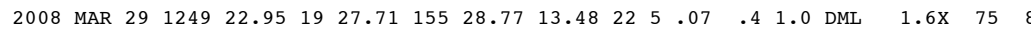

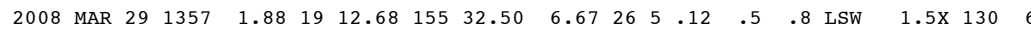

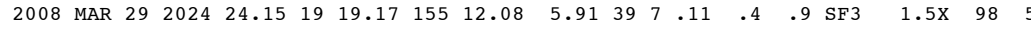

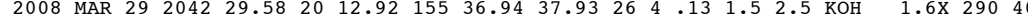

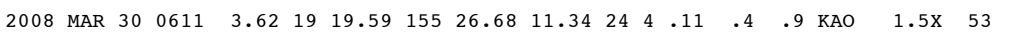


---ORIGIN TIME (HST)-- -LAT N-- --LON W-- DEPTH N N RMS ERH ERZ LOC PREF AZ MIN YEAR MON DA HRMN SEC DEG MIN DEG MIN KM RD S SEC KM KM REMKS MAG GAP DS

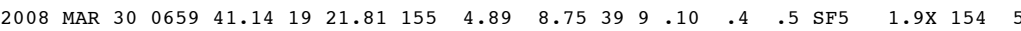

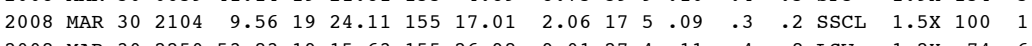

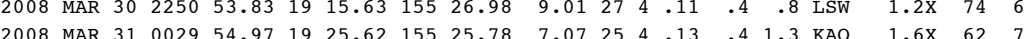

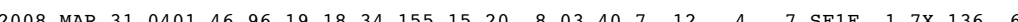

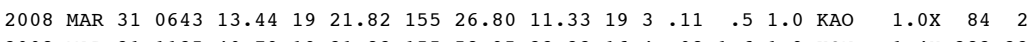

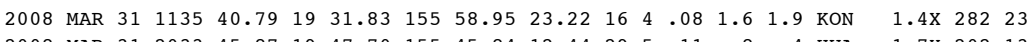

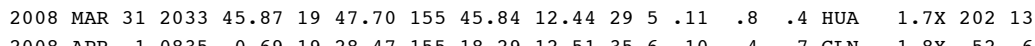

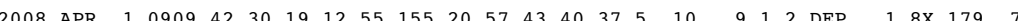

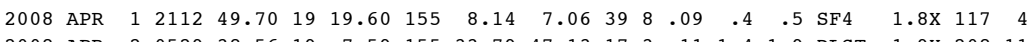

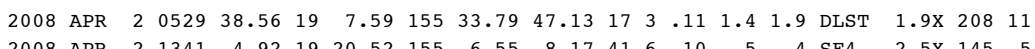

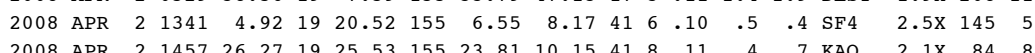
2008 APR $2 \begin{array}{llllllllllllllllll}1616 & 13.38 & 19 & 14.37 & 155 & 8.94 & 42.84 & 33 & 4 & 10 & 1.0 & 1.3 & \text { DEP } & 1.8 \times & 221 & 6\end{array}$

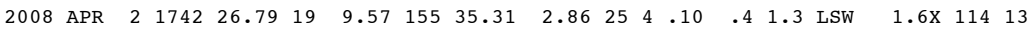

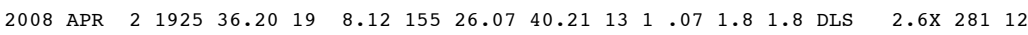

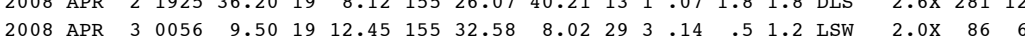

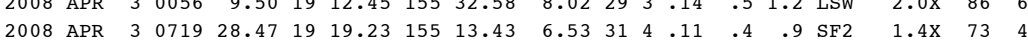

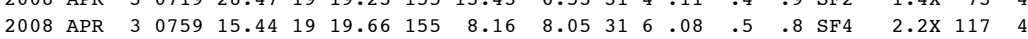

$\begin{array}{lllllllllllllllllll}2008 & \text { APR } & 3 & 1318 & 2.26 & 19 & 19.11 & 155 & 13.59 & 3.97 & 38 & 6 & .11 & .3 & 1.0 & \text { SSF } & 2.1 \mathrm{X} & 68 & 4\end{array}$

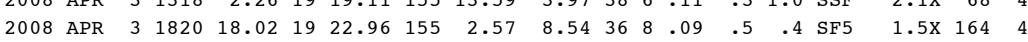

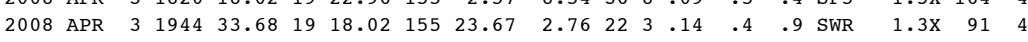

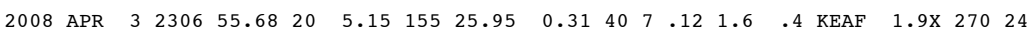

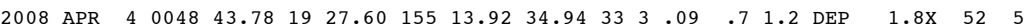

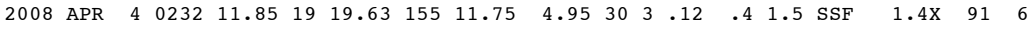

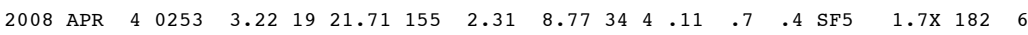
$\begin{array}{lllllllllllllllllll}2008 & \text { APR } & 4 & 0605 & 32.01 & 19 & 6.81 & 155 & 47.90 & 35.05 & 25 & 2 & .10 & 1.1 & 1.8 & \text { KON } & 2.0 \times & 199 & 6\end{array}$ 2008 APR $4 \begin{array}{llllllllllllllll}1630 & 31.61 & 20 & 55.11 & 156 & 23.58 & 9.63 & 44 & 7 & .10 & 1.8 & 1.2 & \text { DISF } & 3.1 \mathrm{X} & 204108\end{array}$

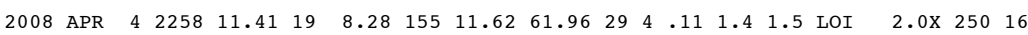

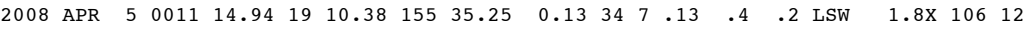
$\begin{array}{lllllllllllllllllll}2008 & \text { APR } & 5 & 0015 & 34.59 & 19 & 27.28 & 155 & 28.54 & 11.62 & 23 & 5 & .09 & .4 & .9 & \text { KAO } & 1.3 \times & 71 & 9\end{array}$

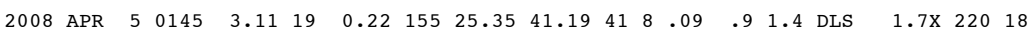
2008 APR $5 \begin{array}{llllllllllllllllll}0503 & 58.66 & 19 & 26.85 & 155 & 28.97 & 10.26 & 41 & 7 & .10 & .3 & .6 & \text { KAO } & 2.0 \mathrm{X} & 54 & 8\end{array}$

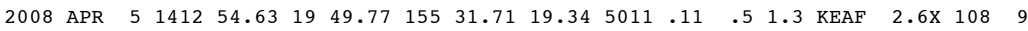

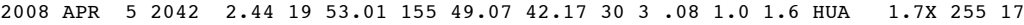

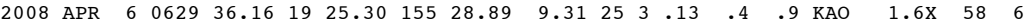
2008 APR $6 \begin{array}{llllllllllllllll}1153 & 57.70 & 19 & 21.43 & 155 & 30.47 & 9.84 & 22 & 1 & .07 & .4 & .9 & \text { KAO } & 1.3 \mathrm{X} & 54 & 5\end{array}$

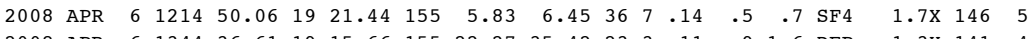

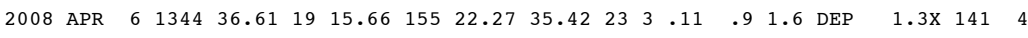

$\begin{array}{lllllllllllllllllll}2008 & \text { APR } & 6 & 1421 & 46.14 & 19 & 15.57 & 155 & 21.64 & 33.73 & 17 & 3 & .11 & 1.3 & 2.1 & \text { DEP } & 1.4 \times & 146 & 5\end{array}$

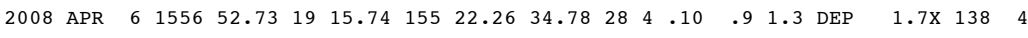

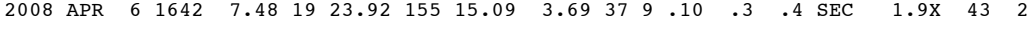

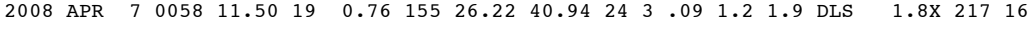

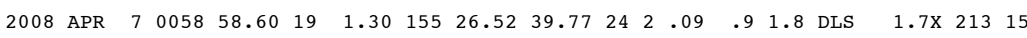

---ORIGIN TTME (HST)-- -LAT N-- --LON W-- DEPTH N N RMS ERH ERZ LOC PREF AZ MIN YEAR MON DA HRMN SEC DEG MIN DEG MIN KM RD S SEC KM KM REMKS MAG GAP DS

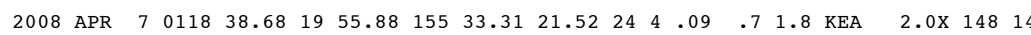

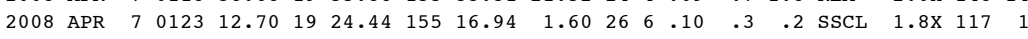

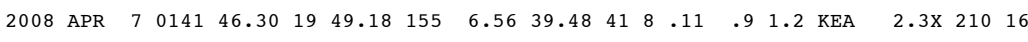
$\begin{array}{lllllllllllllllllll} & \end{array}$

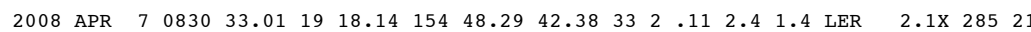

$\begin{array}{lllllllllllllllllll}2008 & \text { APR } & 7 & 2113 & 31.33 & 19 & 19.05 & 155 & 14.89 & 8.15 & 45 & 9 & .12 & .4 & .5 & \text { SF1 } & 2.0 \mathrm{x} & 87 & 4\end{array}$

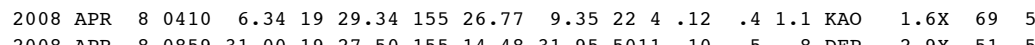
$\begin{array}{lllllllllllllllllll}2008 & \text { APR } & 8 & 0859 & 31.00 & 19 & 27.50 & 155 & 14.48 & 31.95 & 5011 & .10 & .5 & .8 & \text { DEP } & 2.9 \mathrm{X} & 51 & 5 \\ 2008 & \text { APR } & 8 & 1233 & 55.72 & 19 & 24.58 & 155 & 17.19 & 2.06 & 20 & 5 & .11 & .3 & .3 & \text { SNCL } & 1.6 \mathrm{X} & 112 & 2\end{array}$ 2008 APR 8125157.83

$\begin{array}{lllllllllllllllllll}2008 & \text { APR } & 9 & 0110 & 45.28 & 19 & 13.20 & 155 & 34.96 & 34.44 & 33 & 6 & .10 & .8 & 1.2 & \text { DLS } & 1.6 \times & 121 & 9\end{array}$

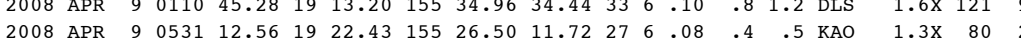

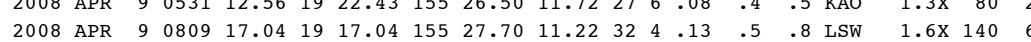

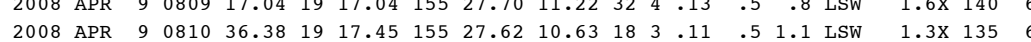
$\begin{array}{llllllllllllllllll}2008 & \text { APR } & 9 & 1010 & 56.41 & 19 & 17.56 & 15 & 13.01 & 6.75 & 25 & 4 & .10 & .5 & 1.0 & \text { SF2 } & 1.5 \times & 178\end{array}$

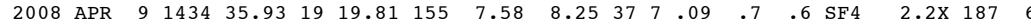

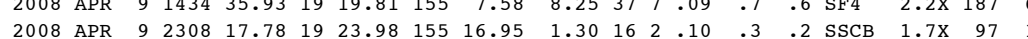
2008 APR $10 \quad \begin{array}{llllllllllllllll}0 & 55.19 & 19 & 21.93 & 15 & 17.06 & 2.23 & 26 & 4 & .10 & .3 & .4 & \text { SWR } & 2.0 \mathrm{x} & 63 & 2\end{array}$

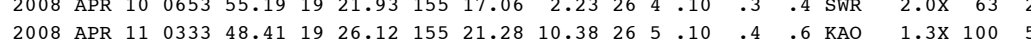

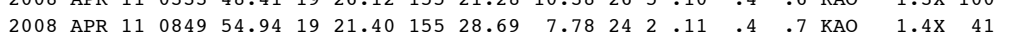

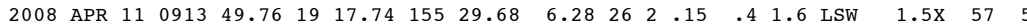

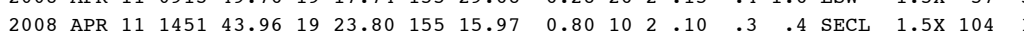

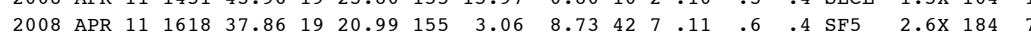

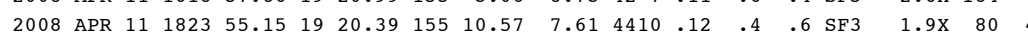

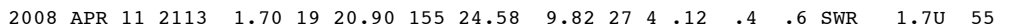

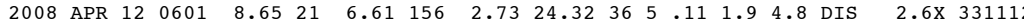

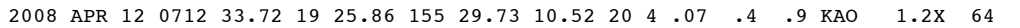

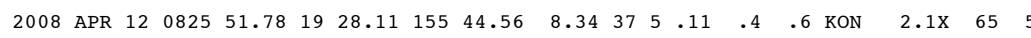

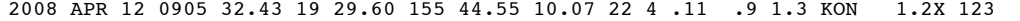

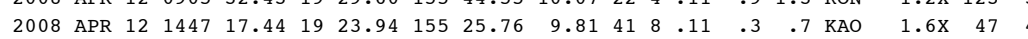

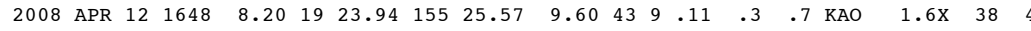

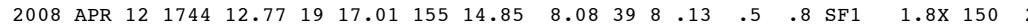

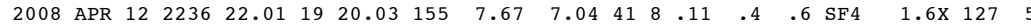

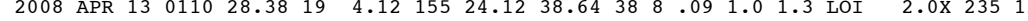

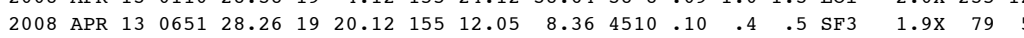

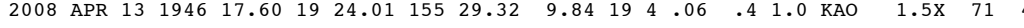

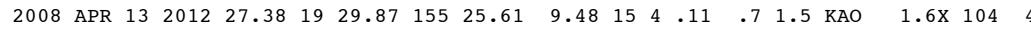

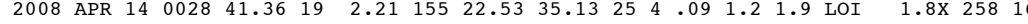

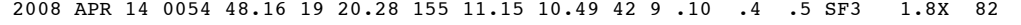

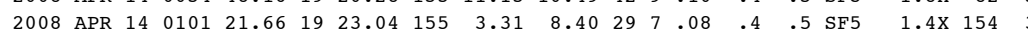

$\begin{array}{lllllllllllllllll}2008 & \text { APR } 14 & 0150 & 49.08 & 19 & 16.00 & 155 & 3.98 & 46.61 & 33 & 5 & .10 & 1.2 & 1.5 & \text { DEP } & 1.7 \times & 227\end{array}$

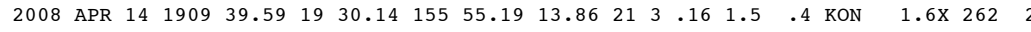

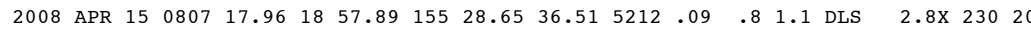

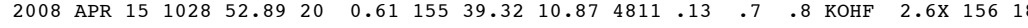

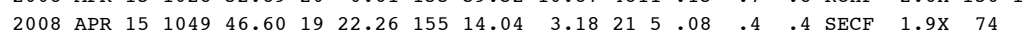


---ORIGIN TIME (HST)-- -LAT N-- --LON W-- DEPTH N N RMS ERH ERZ LOC PREF AZ MIN YEAR MON DA HRMN SEC DEG MIN DEG MIN KM RD S SEC KM KM REMKS MAG GAP DS

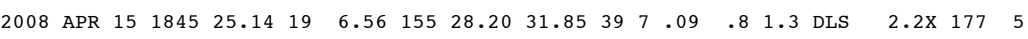
$\begin{array}{lllllllllllllllll}2008 \text { APR } 16 & 0357 & 19.92 & 19 & 24.05 & 155 & 17.02 & 1.46 & 15 & 2 & .06 & .3 & .2 & \text { SSCB } & 1.6 \mathrm{X} & 98 & 1\end{array}$

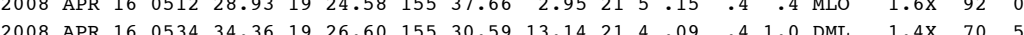

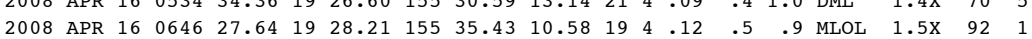

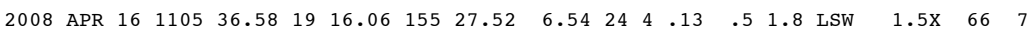

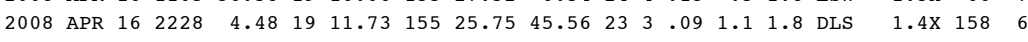
2008 APR $17 \quad \begin{array}{lllllllllllllllll}0.20 & 30.25 & 19 & 10.66 & 155 & 24.61 & 45.34 & 31 & 4 & .12 & 1.0 & 1.5 & \text { DEPT } & 1.9 \times & 165 & 6\end{array}$ 2008 APR $17 \begin{array}{lllllllllllllllllll}0332 & 53.14 & 19 & 30.13 & 155 & 29.63 & 6.40 & 13 & 4 & .13 & .5 & 2.3 & \text { MLO } & 1.4 X & 101 & 5\end{array}$

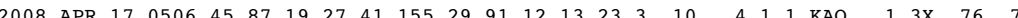

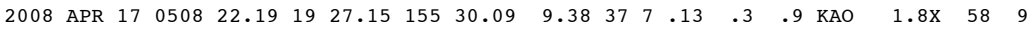

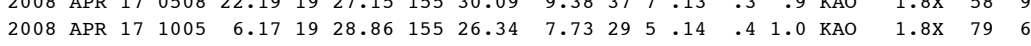

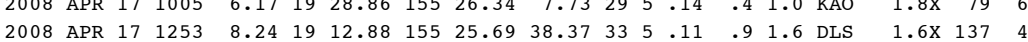

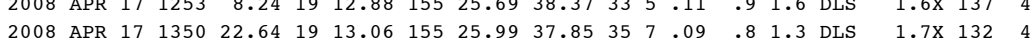

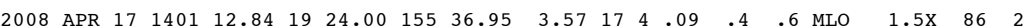

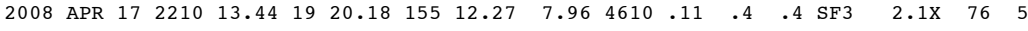

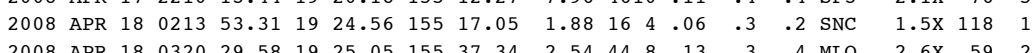

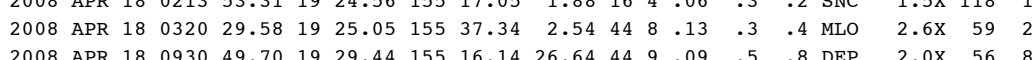

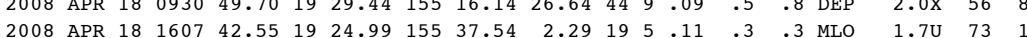

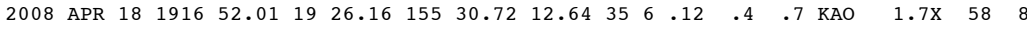

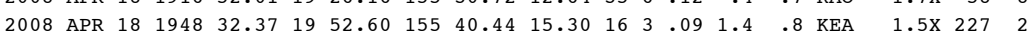

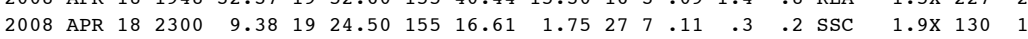

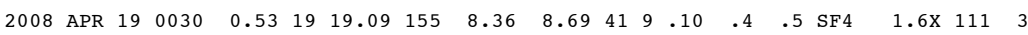

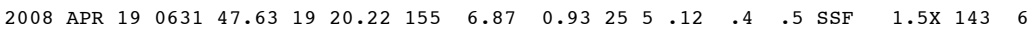

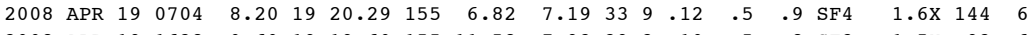

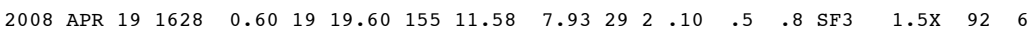

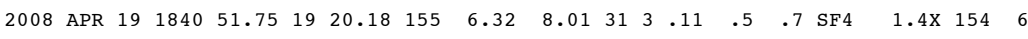

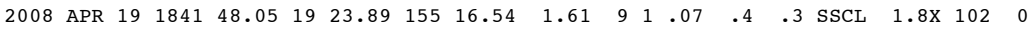

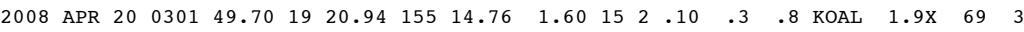

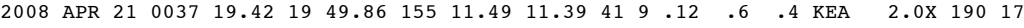

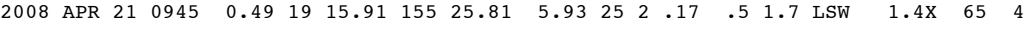

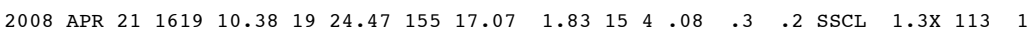

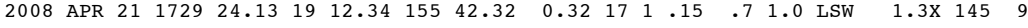
$\begin{array}{lllllllllllllll}2008 & \text { APR } & 21 & 1928 & 6.28 & 19 & 24.83 & 155 & 19.62 & 6.21 & 21 & 5 & .10 & .4 & .9 \\ \text { KAO } & 1.4 \times & 108 & 2\end{array}$

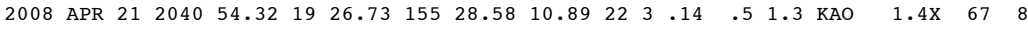

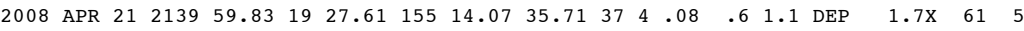

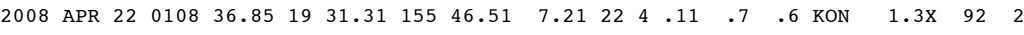

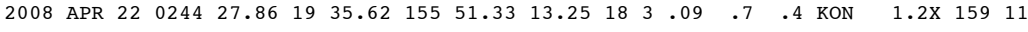

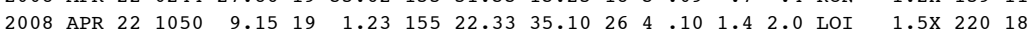

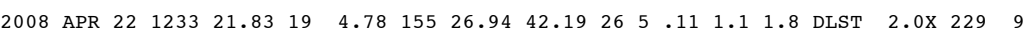

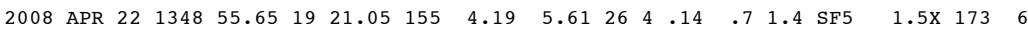

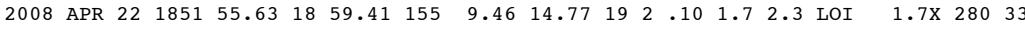

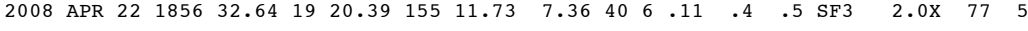

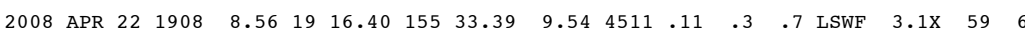

--ORIGIN TIME (HST)-- -LAT N-- --LON W-- DEPTH N N RMS ERH ERZ LOC PREF AZ MIN YEAR MON DA HRMN SEC DEG MIN DEG MIN KM RD S SEC KM KM REMKS MAG GAP DS

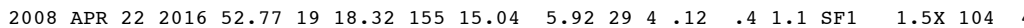

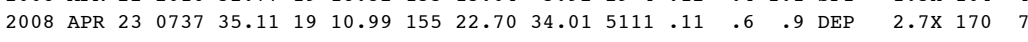

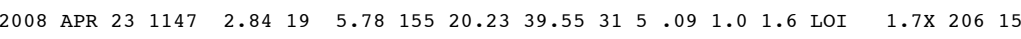

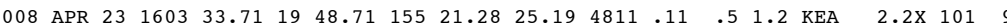

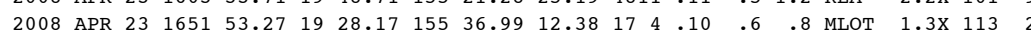

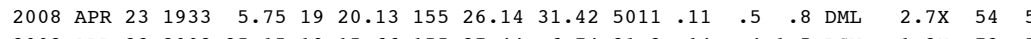

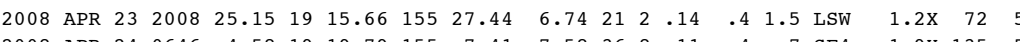

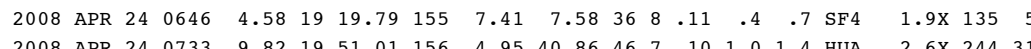

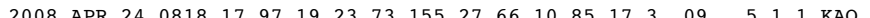

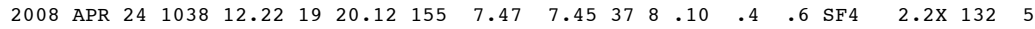

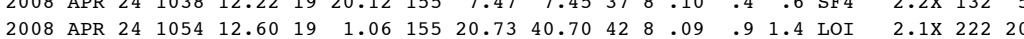

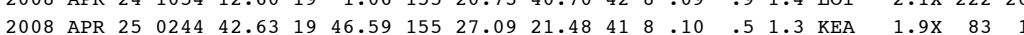

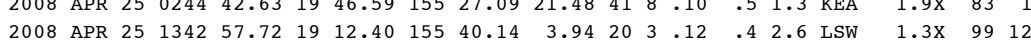

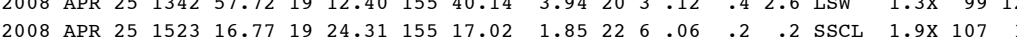

$\begin{array}{llllllllllllllllll}2008 & \text { APR } 25 & 1627 & 57.60 & 19 & 27.39 & 155 & 25.03 & 8.79 & 17 & 4 & .10 & .5 & 1.3 & \text { KAO } & 1.2 \times & 71 & 5\end{array}$

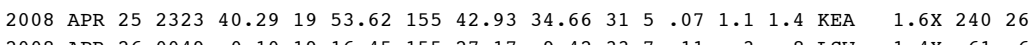

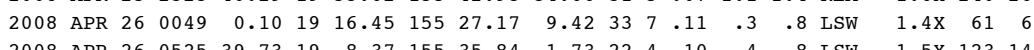

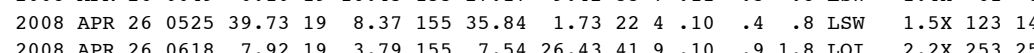

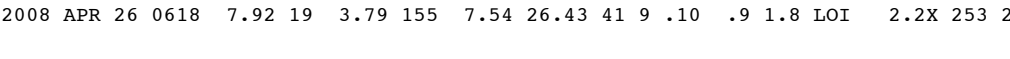
$\begin{array}{lllllllllllllllll}2008 & \text { APR } 26 & 0649 & 37.90 & 19 & 17.27 & 155 & 29.45 & 11.10 & 4410 & .09 & .3 & .6 & \text { LSW } & 2.2 \mathrm{X} & 50 & 4\end{array}$

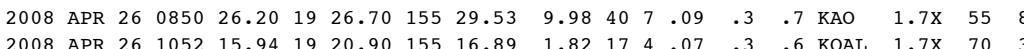

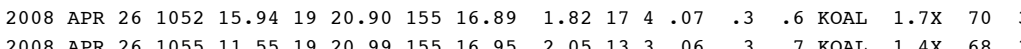

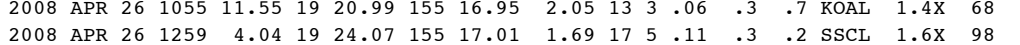

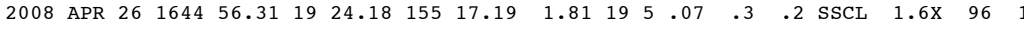
2008 APR $26 \begin{array}{llllllllllllllll}2224 & 59.40 & 19 & 16.34 & 155 & 27.06 & 8.57 & 24 & 1 & .09 & .4 & .6 & \text { LSW } & 1.5 \mathrm{X} & 63\end{array}$

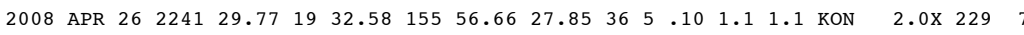

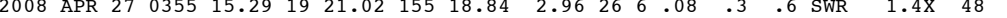

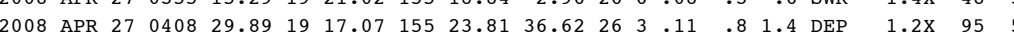

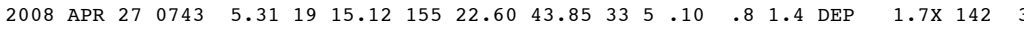

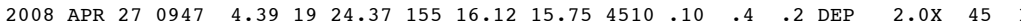

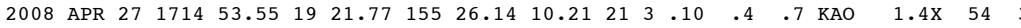

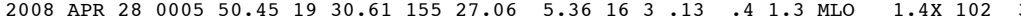

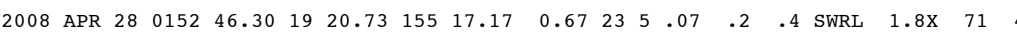

$\begin{array}{lllllllllllllllll}2008 & \text { APR } 28 & 0420 & 21.72 & 19 & 20.92 & 155 & 16.57 & 0.99 & 34 & 3 & .10 & .3 & .5 & \text { KOAL } & 2.1 \mathrm{X} & 72\end{array}$

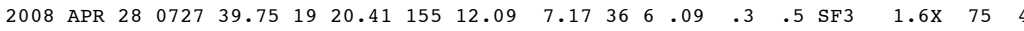

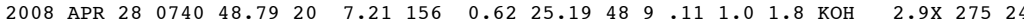

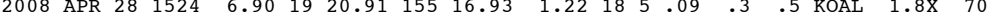

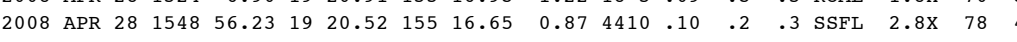

$\begin{array}{lllllllllllllllll}2008 & \text { APR } 28 & 1549 & 23.15 & 19 & 20.61 & 155 & 16.53 & 1.65 & 39 & 7 & .10 & .2 & .4 & \text { KOAL } & 2.6 \mathrm{X} & 76\end{array}$ $\begin{array}{lllllllllllllllll}2008 \text { APR } 28 & 2042 & 58.88 & 19 & 19.51 & 155 & 7.76 & 8.89 & 4510 & .10 & .4 & .5 & \mathrm{SF} 4 & 2.2 \mathrm{X} & 129 & 4\end{array}$

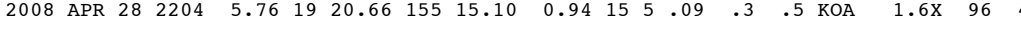

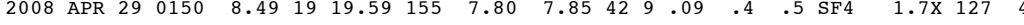

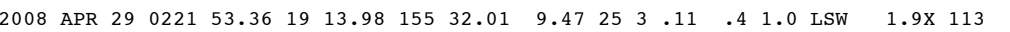


---ORIGIN TIME (HST)-- -LAT N-- --LON W-- DEPTH N N RMS ERH ERZ LOC PREF AZ MIN YEAR MON DA HRMN SEC DEG MIN DEG MIN KM RD S SEC KM KM REMKS MAG GAP DS

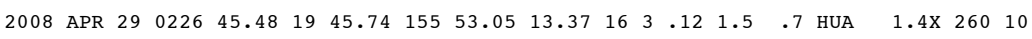

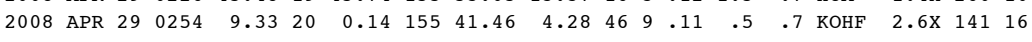

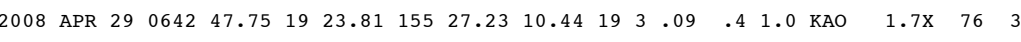

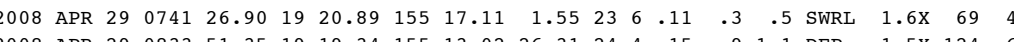

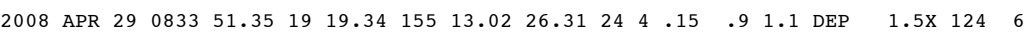

2008 APR $291958 \quad 1.12 \quad 19 \begin{array}{llllllllll}18.67 & 155 & 15.52 & 6.58 & 35 & 5 & .09 & .4 & .8 & \mathrm{SF} 1\end{array}$ $\begin{array}{lllllllllll} & 1.6 \times 102 & 5\end{array}$

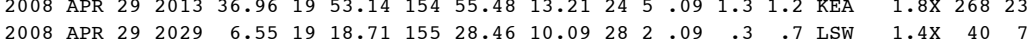

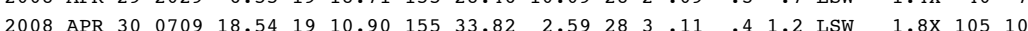

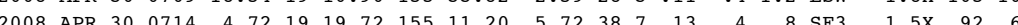

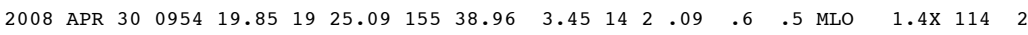

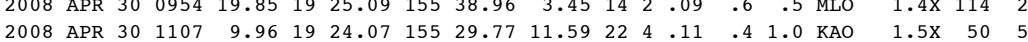

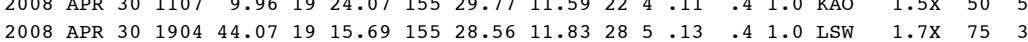

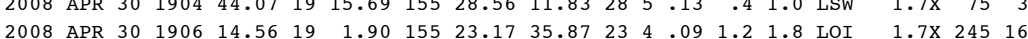
2008 MAY $1 \quad \begin{array}{lllllllllllllllllll}0 & 0231 & 28.22 & 19 & 15.47 & 155 & 28.01 & 7.18 & 32 & 4 & .16 & .4 & 1.1 & \text { LSW } & 1.5 X & 72 & 4\end{array}$

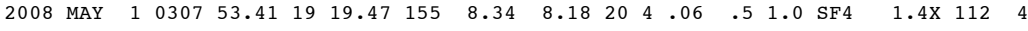

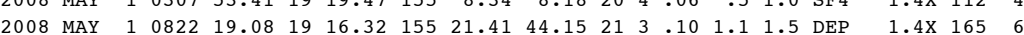

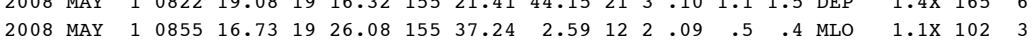

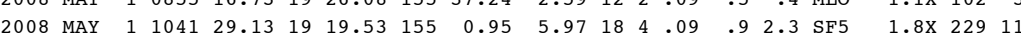

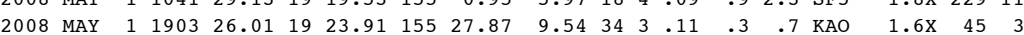

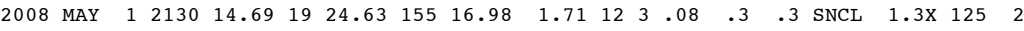

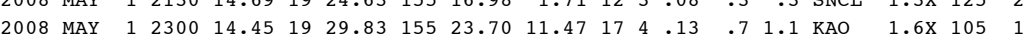

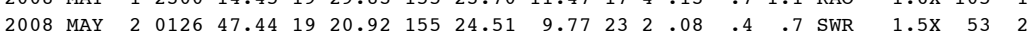

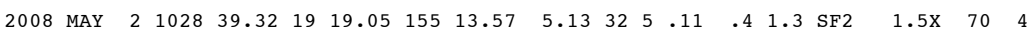

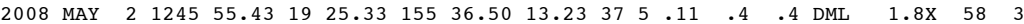

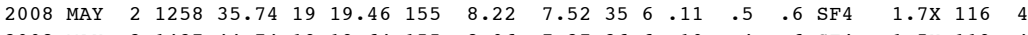
$\begin{array}{lllllllllllllllllll}2008 & \text { MAY } & 2 & 1437 & 44.74 & 19 & 19.64 & 155 & 8.06 & 7.27 & 36 & 6 & .10 & .4 & .6 & \mathrm{SF} 4 & 1.5 \mathrm{X} & 119 & 4\end{array}$ $\begin{array}{llllllllllllllllll}2008 & \text { MAY } & 2 & 1518 & 32.06 & 19 & 37.60 & 155 & 16.35 & 38.18 & 4611 & .10 & .6 & 1.0 & \text { KEA } & 2.0 X & 85 & 19\end{array}$

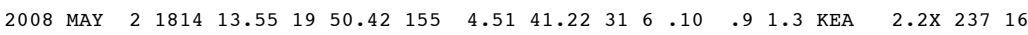

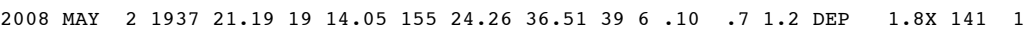

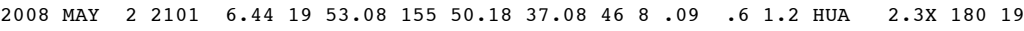

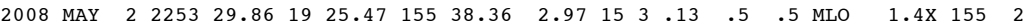

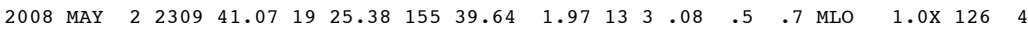

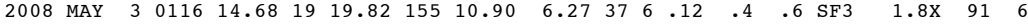

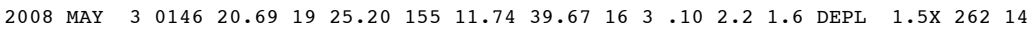

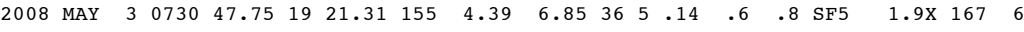

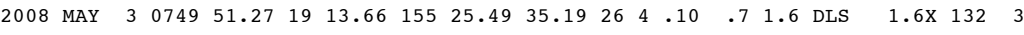
$\begin{array}{llllllllllllllllllll}2008 & \text { MAY } & 3 & 0832 & 39.51 & 19 & 21.45 & 155 & 33.40 & 5.54 & 16 & 1 & .12 & .5 & 3.1 & \text { MLO } & 1.2 \mathrm{X} & 73 & 6\end{array}$ $\begin{array}{lllllllllllllllllll}2008 & \text { MAY } & 3 & 0936 & 31.19 & 19 & 33.75 & 155 & 37.26 & 11.67 & 19 & 4 & .10 & .6 & 1.2 & \text { MLO } & 1.3 \times & 182 & 9\end{array}$ $\begin{array}{lllllllllllllllllll}2008 & \text { MAY } & 3 & 1237 & 20.78 & 19 & 20.04 & 155 & 12.77 & 8.53 & 31 & 5 & .10 & .4 & .8 & \mathrm{SF} 2 & 1.2 \mathrm{X} & 73 & 5\end{array}$

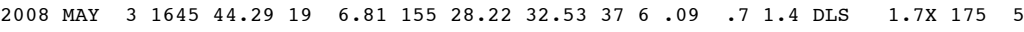
$\begin{array}{lllllllllllllllllll}2008 & \text { MAY } & 3 & 2256 & 17.45 & 19 & 18.43 & 155 & 15.46 & 8.19 & 42 & 9 & .12 & .4 & .6 & \mathrm{SF} 1 & 1.5 \times & 107 & 4\end{array}$

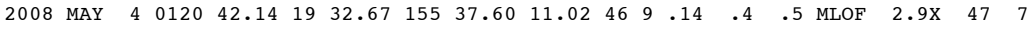

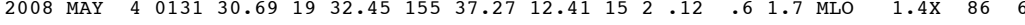

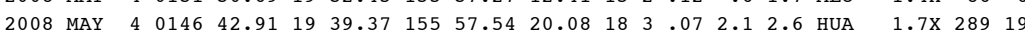

---ORIGIN TTME (HST)-- -LAT N-- --LON W-- DEPTH N N RMS ERH ERZ LOC PREF AZ MIN YEAR MON DA HRMN SEC DEG MIN DEG MIN KM RD S SEC KM KM REMKS MAG GAP DS

2008 MAY $4 \quad 0256 \quad 19.85 \quad 19 \quad 32.45 \quad 155 \quad 37.57 \quad 10.84 \quad 27 \quad 5 \quad 14 \quad 6 \quad 1.2$ MLO $\quad 1.5 \times \quad 86 \quad 6$ $\begin{array}{lllllllllllllllllll}2008 & \text { MAY } & 4 & 0419 & 59.92 & 19 & 34.98 & 155 & 42.81 & 2.43 & 20 & 4 & .10 & .4 & 1.0 & \text { MLO } & 1.1 \mathrm{X} & 96 & 10\end{array}$

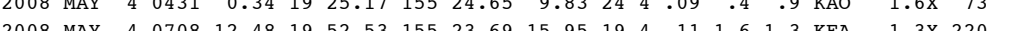

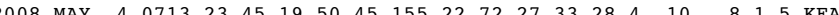

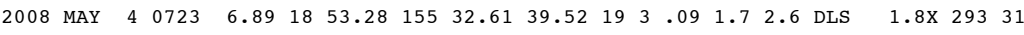

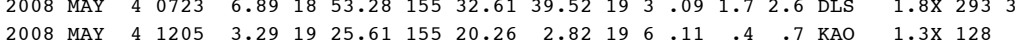

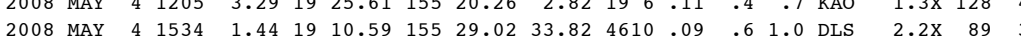

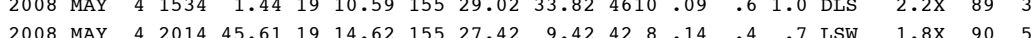

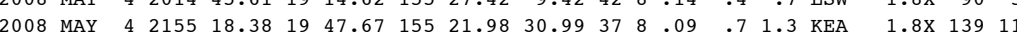

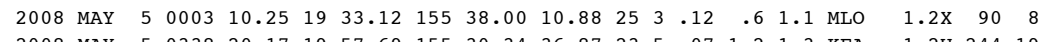

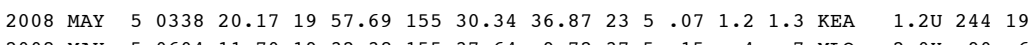

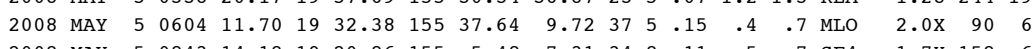

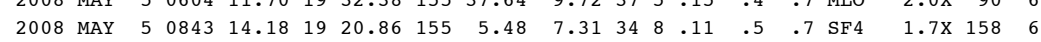

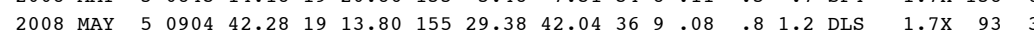

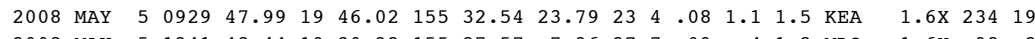

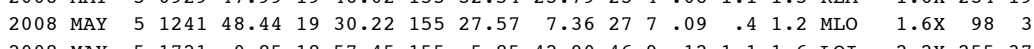

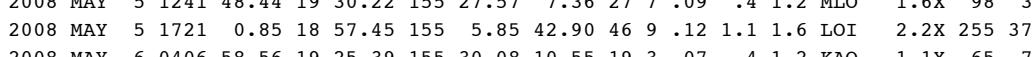

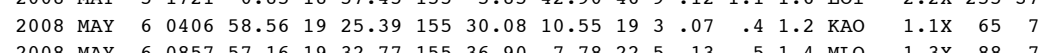

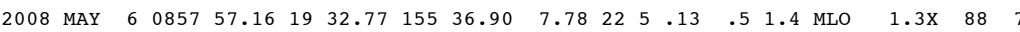

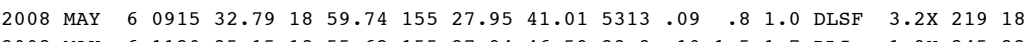

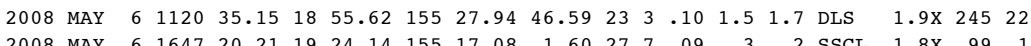

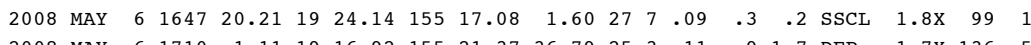

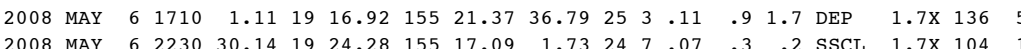

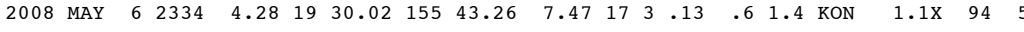

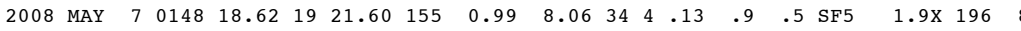

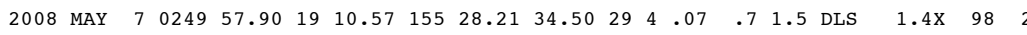

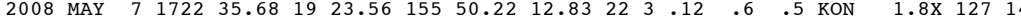

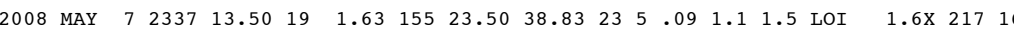

$\begin{array}{lllllllllllllllllll}2008 & \text { MAY } & 8 & 0334 & 18.72 & 19 & 18.90 & 155 & 12.89 & 5.55 & 44 & 9 & .11 & .4 & .7 & \text { SF2 } & 1.9 X & 89 & 4\end{array}$ 2008 MAY $8 \begin{array}{lllllllllllllllll} & 0342 & 0.56 & 19 & 18.90 & 155 & 12.80 & 5.33 & 44 & 8 & .12 & .4 & .6 & \mathrm{SF} 2 & 2.1 \mathrm{X} & 91 & 4\end{array}$

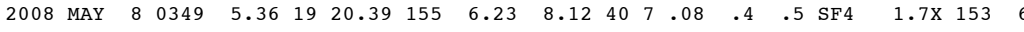
$\begin{array}{llllllllllllllllll}2008 & \text { MAY } & 8 & 0409 & 51.32 & 19 & 18.56 & 155 & 12.95 & 9.54 & 40 & 8 & .10 & .4 & .6 & \text { SF2 } & 2.1 \mathrm{X} & 94\end{array}$

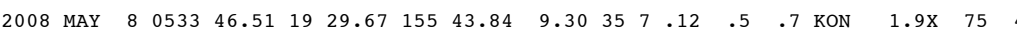

$\begin{array}{lllllllllllllllllll}2008 & \text { MAY } & 8 & 1324 & 52.06 & 19 & 28.81 & 155 & 26.62 & 6.75 & 35 & 6 & .11 & .3 & 1.0 & \text { KAO } & 1.6 \mathrm{X} & 69 & 6\end{array}$

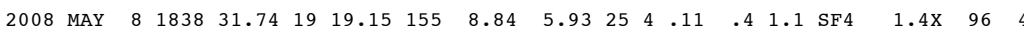

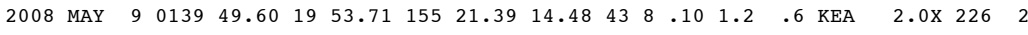

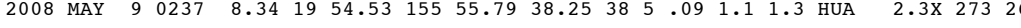

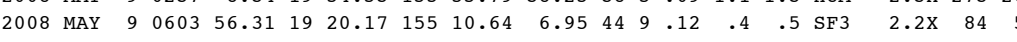

$\begin{array}{lllllllllllllllllll}2008 & \text { MAY } & 9 & 0808 & 32.52 & 19 & 23.72 & 155 & 15.38 & 2.86 & 16 & 4 & .07 & .4 & .4 & \text { SECL } & 1.6 X & 97 & 2\end{array}$ $\begin{array}{llllllllllllllllll}2008 & \text { MAY } & 9 & 0858 & 8.94 & 19 & 18.54 & 155 & 15.06 & 6.38 & 23 & 3 & .11 & .5 & 1.3 & \text { SF1 } & 1.3 \times & 109\end{array}$

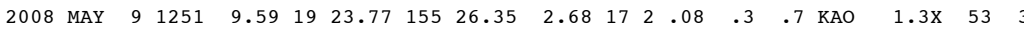

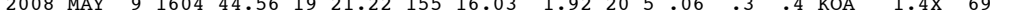

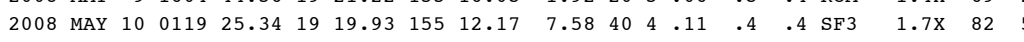


---ORIGIN TIME (HST)-- -LAT N-- --LON W-- DEPTH N N RMS ERH ERZ LOC PREF AZ MIN YEAR MON DA HRMN SEC DEG MIN DEG MIN KM RD S SEC KM KM REMKS MAG GAP DS $\begin{array}{lllllllllllllllllll}2008 & \text { MAY } 10 & 0203 & 28.37 & 19 & 25.70 & 155 & 27.83 & 7.48 & 14 & 3 & .11 & .5 & 1.4 & \text { KAO } & 1.1 U & 85 & 6\end{array}$

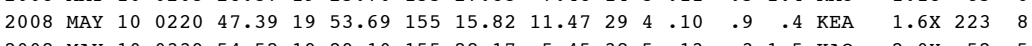

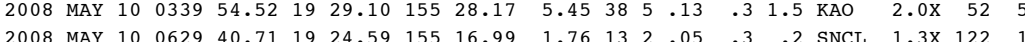

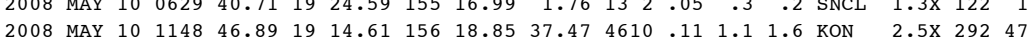

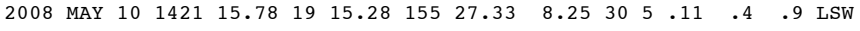
2008 MAY $10 \quad 1430 \quad 50.52 \quad 18 \quad 51.49 \quad 155 \quad 10.29 \quad 32.03 \quad 44 \quad 9.12 \quad 1.1 \quad 2.5$ LOI 2008 MAY $10 \quad 200251.01 \quad 1929.38 \quad 15526.25 \quad 8.22 \quad 8 \quad 3 \quad .05 \quad .92 .0$ LOI

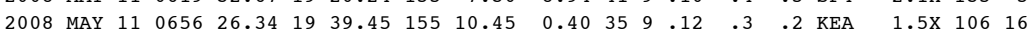

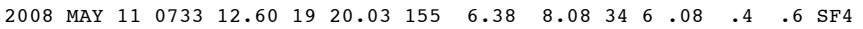

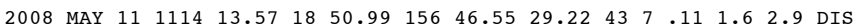

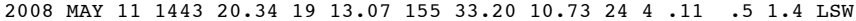

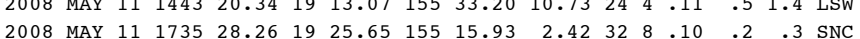

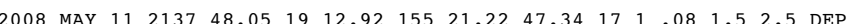

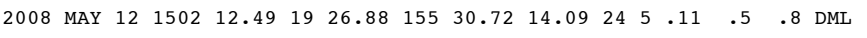

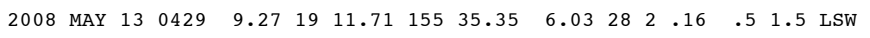
2008 MAY $13 \quad 0534 \quad 48.83 \quad 19 \quad 4.87 \quad 156 \quad 16.20 \quad 41.62 \quad 23 \quad 4.11 \quad 1.6 \quad 2.5$ KON

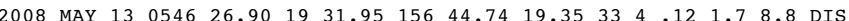

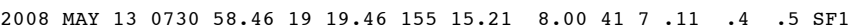

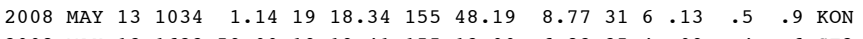

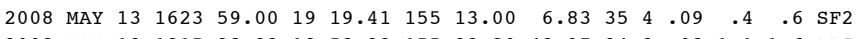

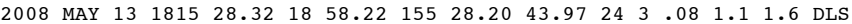

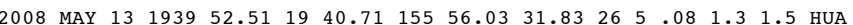

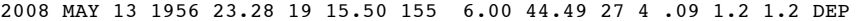

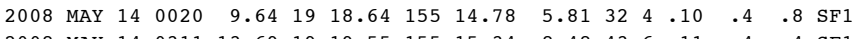

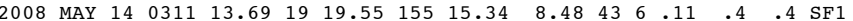

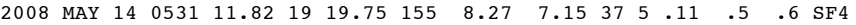

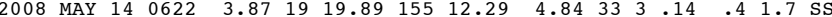

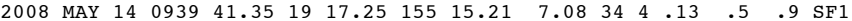

2008 MAY $14 \quad 1109 \begin{array}{llllllllllll}16.94 & 19 & 23.36 & 155 & 29.91 & 11.48 & 20 & 3 & .12 & .5 & 1.0 & \text { KAO }\end{array}$

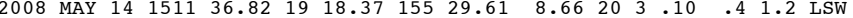

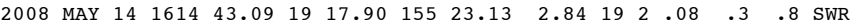

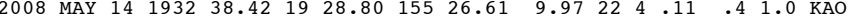

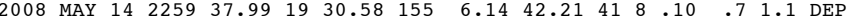

2008 MAY $150348 \begin{array}{llllllllllll}39.85 & 19 & 12.94 & 155 & 29.74 & 9.76 & 25 & 4 & .11 & .4 & .5 & \text { LSW }\end{array}$

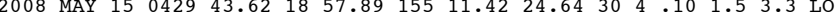

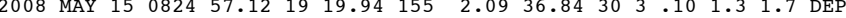

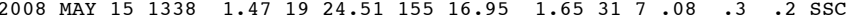

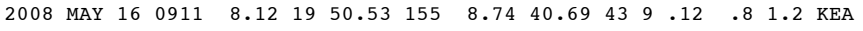

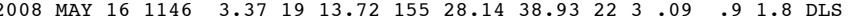
$\begin{array}{lllllllllllllll}2008 \text { MAY } & 16 & 1848 & 41.05 & 19 & 27.07 & 155 & 30.07 & 12.39 & 24 & 3 & .11 & .4 & .9 & \text { KAO }\end{array}$

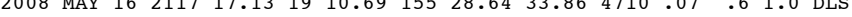
$\begin{array}{llllllllllllllllll}2008 & \text { MAY } & 17 & 0200 & 23.00 & 19 & 20.45 & 155 & 7.22 & 8.55 & 37 & 8 & .09 & .4 & .4 & \text { SF } 4 & 1.5 \times & 134 \\ 2008 & \text { MAY } & 17 & 0532 & 42.31 & 19 & 13.66 & 155 & 35.15 & 7.26 & 31 & 4 & .12 & .4 & 1.2 & \text { LSW } & 2.0 x & 78\end{array}$

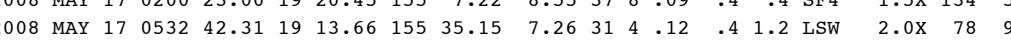

--ORIGIN TIME (HST)-- -LAT N-- --LON W-- DEPTH N N RMS ERH ERZ LOC PREF AZ MTN YEAR MON DA HRMN SEC DEG MIN DEG MIN KM RD S SEC KM KM REMKS MAG GAP DS

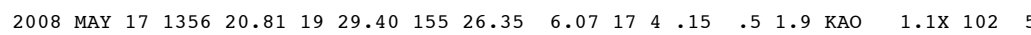

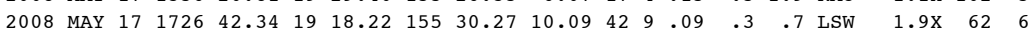

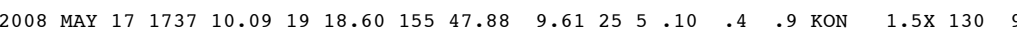

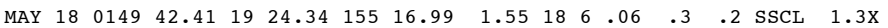

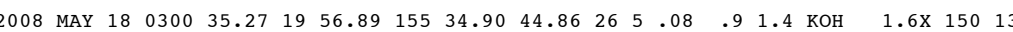

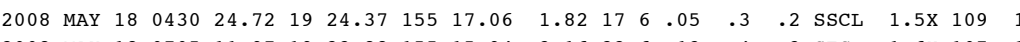

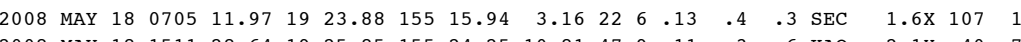

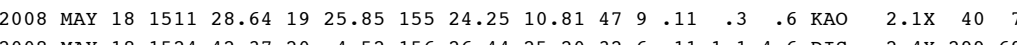

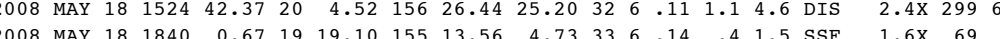

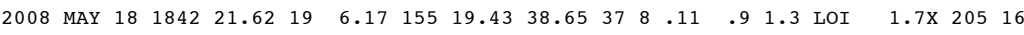

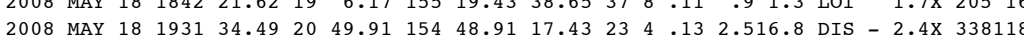

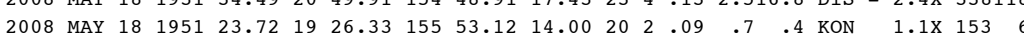

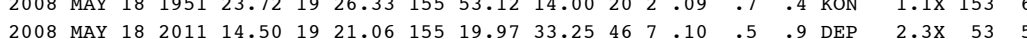

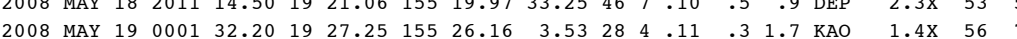

$\begin{array}{lllllllllllllllllll}2008 & \text { MAY } & 19 & 0356 & 38.43 & 19 & 22.23 & 155 & 14.22 & 1.88 & 17 & 3 & .05 & .3 & .3 & \text { SECL } & 1.6 \mathrm{X} & 71 & 2\end{array}$

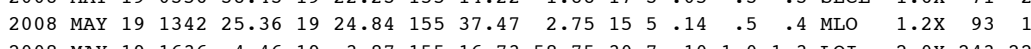

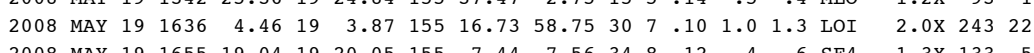

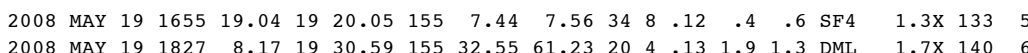

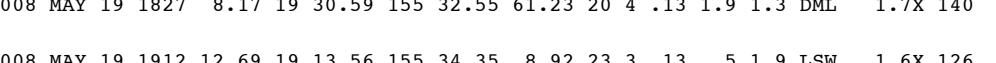

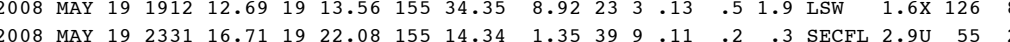

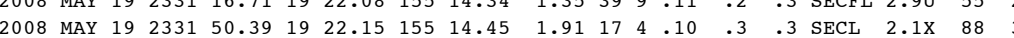

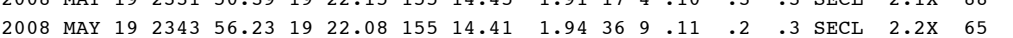

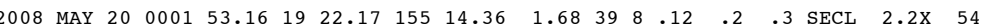

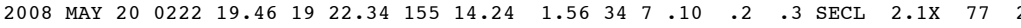

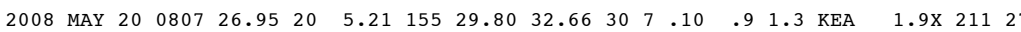

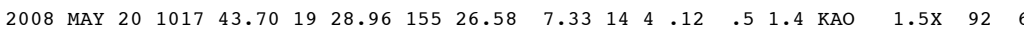

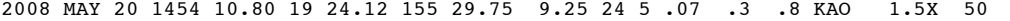

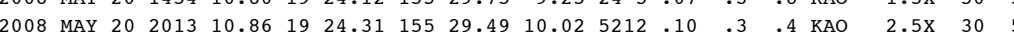

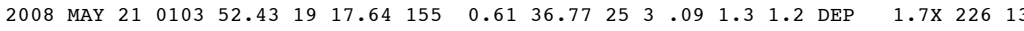

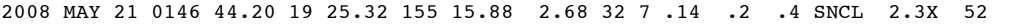

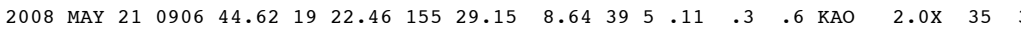

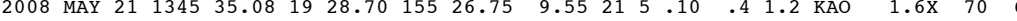

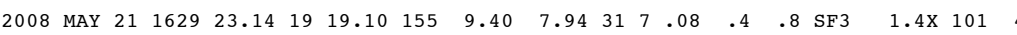

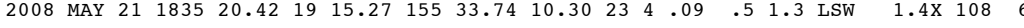

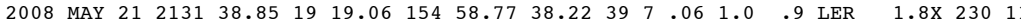

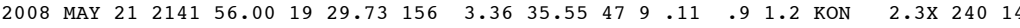

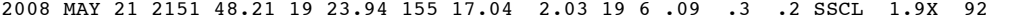

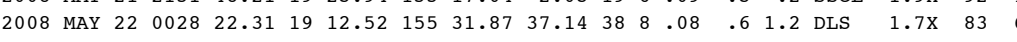

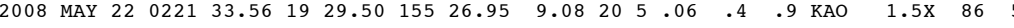

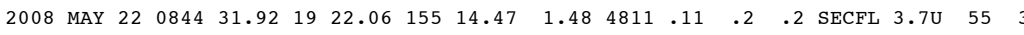

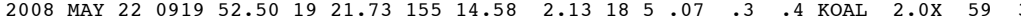

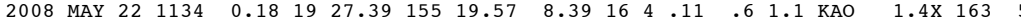

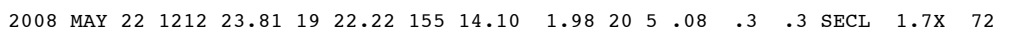


---ORIGIN TIME (HST)-- -LAT N-- --LON W-- DEPTH N N RMS ERH ERZ LOC PREF AZ MIN YEAR MON DA HRMN SEC DEG MIN DEG MIN KM RD S SEC KM KM REMKS MAG GAP DS $\begin{array}{llllllllllllllllll}2008 & \text { MAY } 22 & 1709 & 33.64 & 19 & 21.63 & 155 & 46.66 & 13.63 & 19 & 4 & .08 & .5 & .4 & \text { KON } & 1.2 \times & 140 & 12\end{array}$

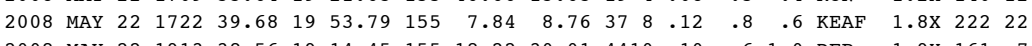

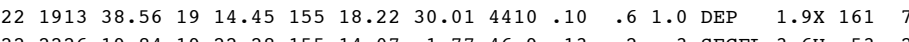

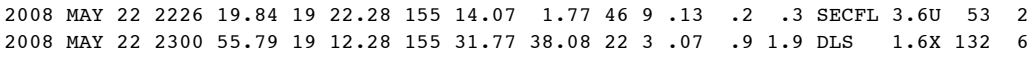

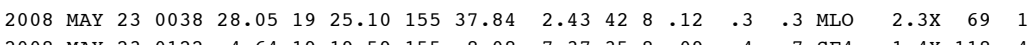

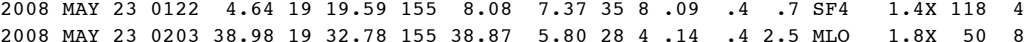

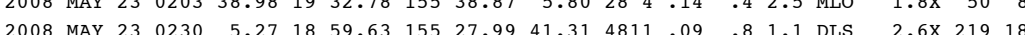

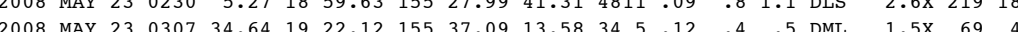

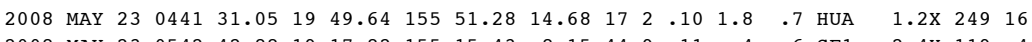

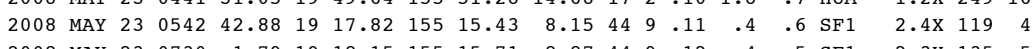

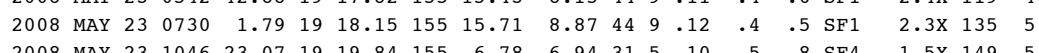

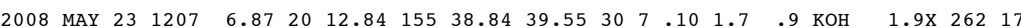

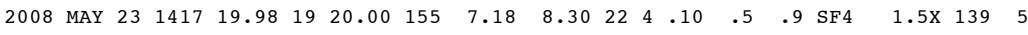

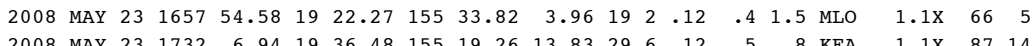

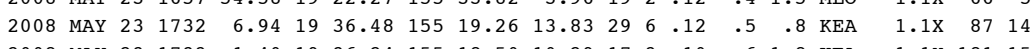

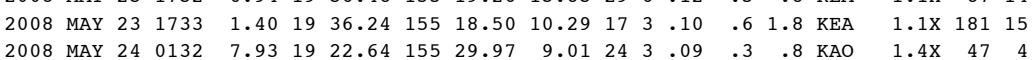

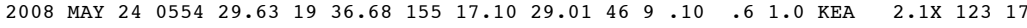

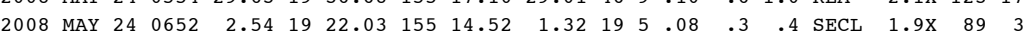

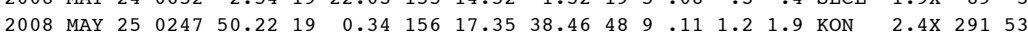

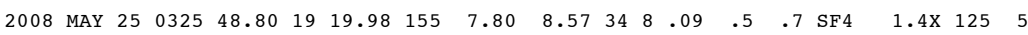

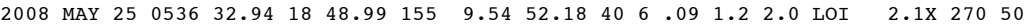

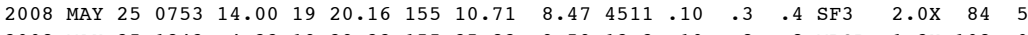

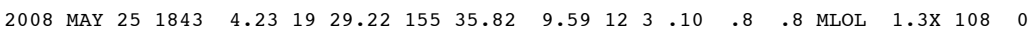

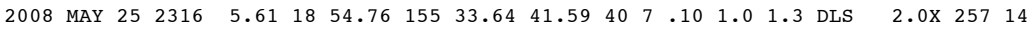

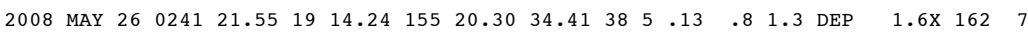

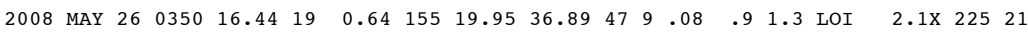

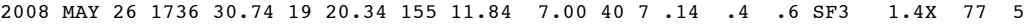

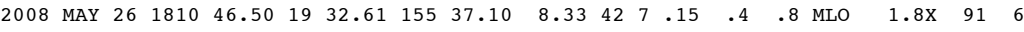
2008 MAY $261847 \begin{array}{lllllllllllllll}19.44 & 19 & 18.68 & 155 & 14.99 & 6.79 & 33 & 5 & .10 & .4 & .8 & \mathrm{SF} 1 & 1.4 \mathrm{X} & 96 & 4\end{array}$

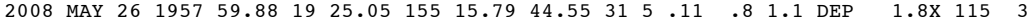

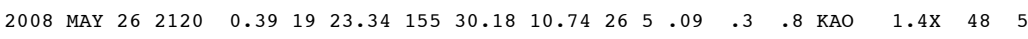

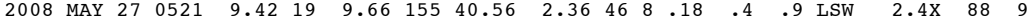

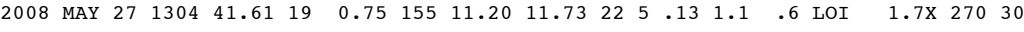

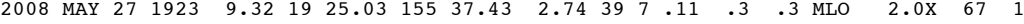
2008 MAY $28 \quad \begin{array}{lllllllllllllllll}0349 & 27.82 & 19 & 25.72 & 155 & 37.63 & 3.46 & 39 & 9 & .12 & .3 & .5 & \text { MLO } & 2.1 \mathrm{X} & 81 & 3\end{array}$

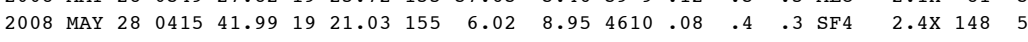

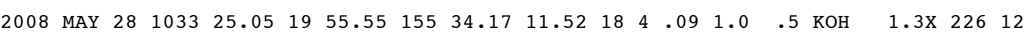

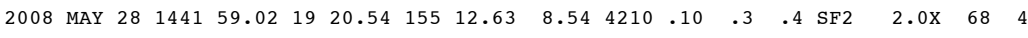

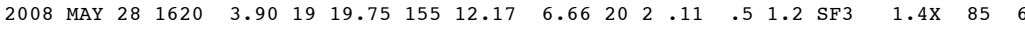

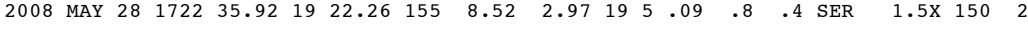

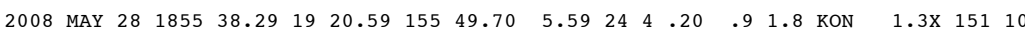

---ORTGIN TIME (HST)-- -LAT N-- --LON W-- DEPTH N N RMS ERH ERZ LOC PREF AZ MIN YEAR MON DA HRMN SEC DEG MIN DEG MIN KM RD S SEC KM KM REMRS MAG GAP DS

2008 MAY $28 \quad 1915 \quad 5.38 \quad 18 \quad 58.70 \quad 155 \quad 28.29 \quad 42.62 \quad 40 \quad 6 \quad 08 \quad 9 \quad 1.2$ DLS $\quad 1.9 X \quad 226 \quad 20$

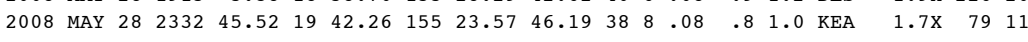

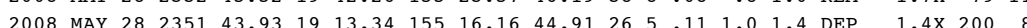

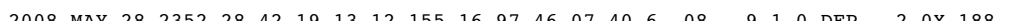

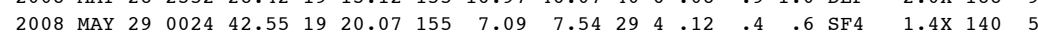

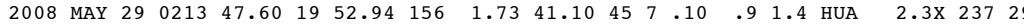

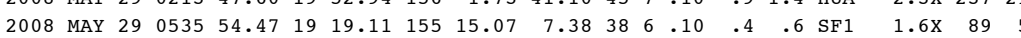

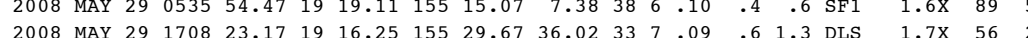

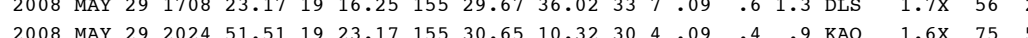

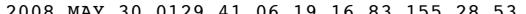

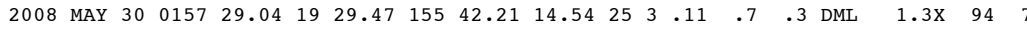

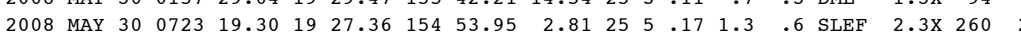

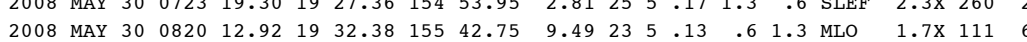

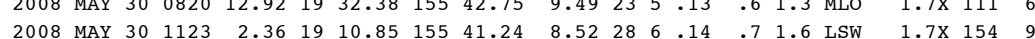

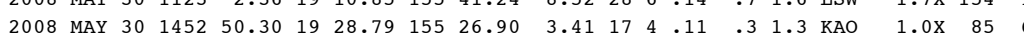

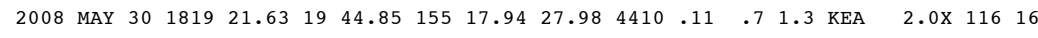

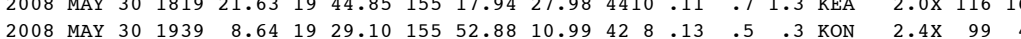

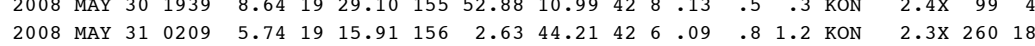

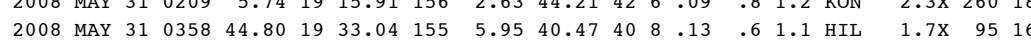

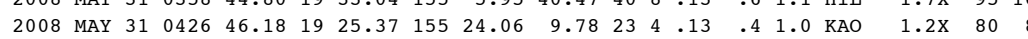

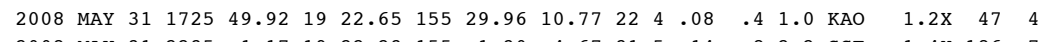

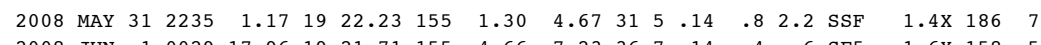

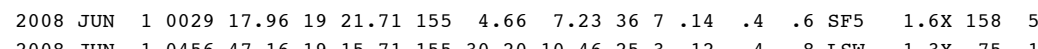

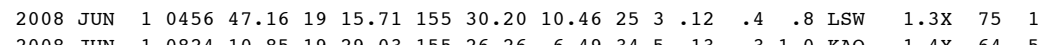

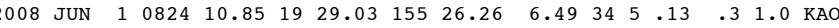

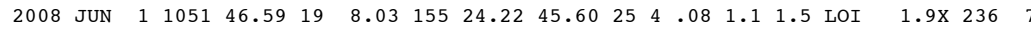

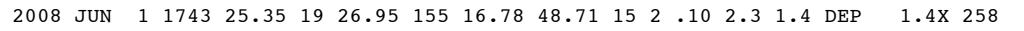

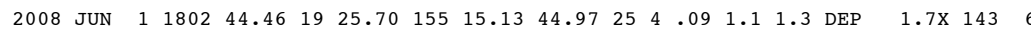

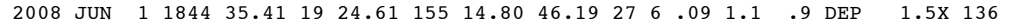

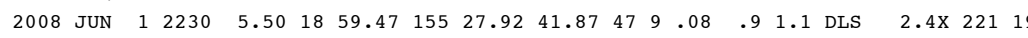

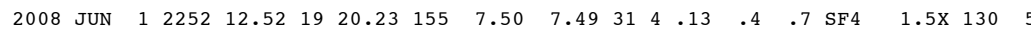

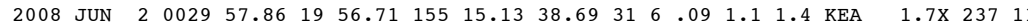

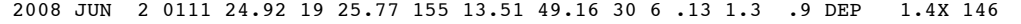

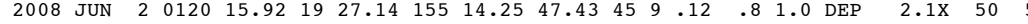

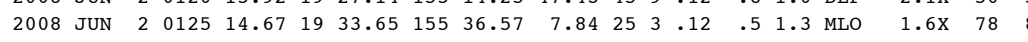

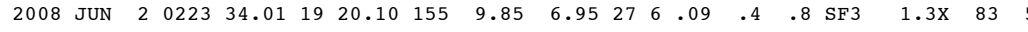

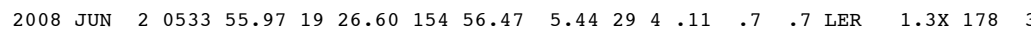

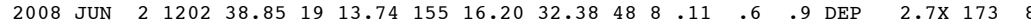

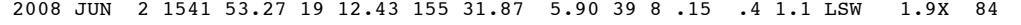

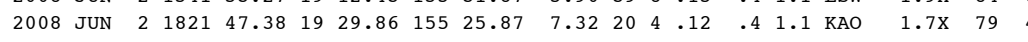

$\begin{array}{llllllllllllllllll}2008 & \text { JUN } & 2 & 1822 & 40.99 & 19 & 23.06 & 155 & 27.29 & 9.44 & 38 & 5 & .10 & .3 & .5 & \text { KAO } & 1.5 \times & 33\end{array}$ $\begin{array}{lllllllllllllllllll}2008 & \text { JUN } & 3 & 0716 & 37.23 & 19 & 20.21 & 155 & 13.54 & 8.19 & 30 & 6 & .09 & .4 & .7 & \mathrm{SF} 2 & 1.4 \mathrm{x} & 62 & 5\end{array}$

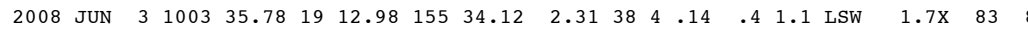

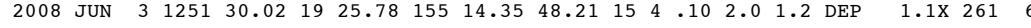

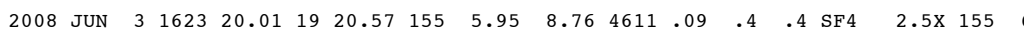


---ORIGIN TIME (HST)-- -LAT N-- --LON W-- DEPTH N N RMS ERH ERZ LOC PREF AZ MIN YEAR MON DA HRMN SEC DEG MIN DEG MIN KM RD S SEC KM KM REMKS MAG GAP DS

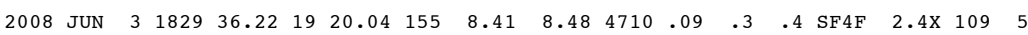
2008 JUN $3 \begin{array}{llllllllllllllll}1830 & 41.76 & 19 & 19.83 & 155 & 8.48 & 8.57 & 37 & 9 & .09 & .4 & .6 & \text { SF } 4 & 1.8 \times & 108 & 5\end{array}$

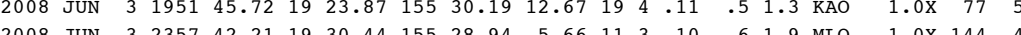

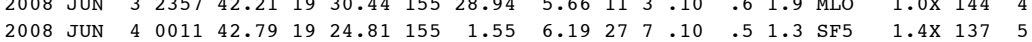

2008 JUN $4 \begin{array}{lllllllllllllllll}4 & 0144 & 49.57 & 19 & 7.34 & 155 & 28.36 & 30.77 & 27 & 4 & .09 & .8 & 1.5 & \text { DLS } & 1.5 \times & 179 & 4\end{array}$ \begin{tabular}{llllllllllllllllll}
2008 & \\
\hline
\end{tabular}

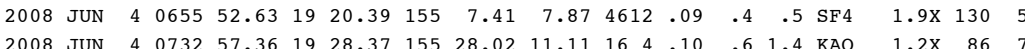
$\begin{array}{llllllllllllllll}2008 & & \end{array}$

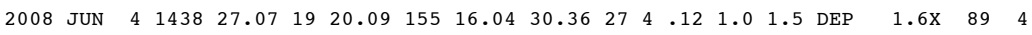

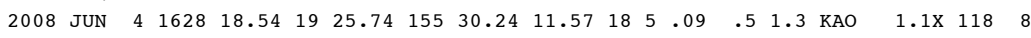

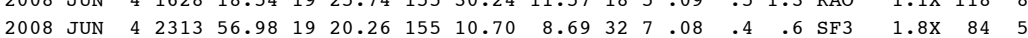

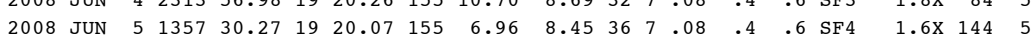
$\begin{array}{lllllllllllllllllll}2008 & \text { JUN } & 5 & 1718 & 25.77 & 19 & 21.80 & 155 & 26.61 & 10.89 & 31 & 5 & .10 & .4 & .7 & \text { KAO } & 1.2 \mathrm{X} & 46 & 2\end{array}$

2008 JUN $5 \begin{array}{lllllllllllllllll}5 & 2229 & 31.58 & 19 & 12.51 & 155 & 31.93 & 37.17 & 46 & 9 & .08 & .7 & 1.0 & \text { DLS } & 2.1 \times & 84 & 6\end{array}$

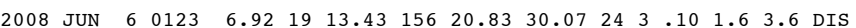
2008 JUN $6 \begin{array}{llllllllllllll}6 & 0158 & 1.23 & 19 & 39.52 & 156 & 12.75 & 30.61 & 30 & 6 & .12 & 1.3 & 2.6 & \text { HUA }\end{array}$ 2008 JUN $6 \quad \begin{array}{lllllllllllllll}0 & 07436.88 & 36 & 19.92 & 155 & 3.96 & 6.38 & 34 & 9 & .12 & .6 & 1.4 & \text { HF5 }\end{array}$ 2008 JUN $6 \begin{array}{llllllllllllll}6 & 1525 & 12.54 & 18 & 59.76 & 155 & 25.92 & 42.29 & 30 & 6 & .08 & 1.1 & 1.7 & \text { DLS }\end{array}$

2008 JUN $6 \begin{array}{lllllllllllll}1541 & 20.25 & 18 & 58.93 & 155 & 25.68 & 41.07 & 25 & 5 & .11 & 1.4 & 2.0 & \text { DLS }\end{array}$ 2008 JUN $6 \begin{array}{llllllllllllll}6 & 1703 & 25.28 & 18 & 52.54 & 155 & 9.81 & 43.78 & 31 & 6 & .09 & 1.3 & 2.3 & \text { LOI }\end{array}$ 2008 JUN $6 \begin{array}{lllllllllllll}1833 & 50.07 & 19 & 25.00 & 155 & 39.14 & 2.97 & 15 & 3 & .08 & .9 & & 6\end{array}$ MLO

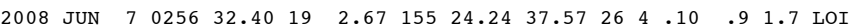
2008 JUN $7 \begin{array}{llllllllllllll}0627 & 3.51 & 19 & 25.83 & 155 & 28.94 & 10.59 & 19 & 4 & .09 & .4 & 1.2 & \text { KAO }\end{array}$

2008 JUN $\quad \begin{array}{llllllllllllll}7 & 1300 & 0.27 & 19 & 19.86 & 155 & 6.43 & 8.96 & 34 & 8 & .09 & .4 & .5 & \mathrm{SF} 4\end{array}$

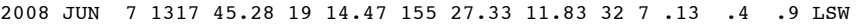
2008 JUN $7 \begin{array}{lllllllllllll}1509 & 45.13 & 19 & 24.06 & 155 & 16.00 & 1.86 & 15 & 4 & .14 & .3 & .4 & \text { SECL }\end{array}$

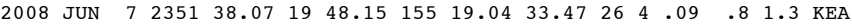

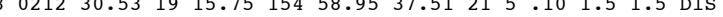

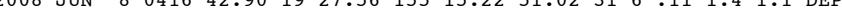
$\begin{array}{llllllllllllllll}2008 & \text { JUN } & 8 & 0452 & 32.60 & 19 & 24.70 & 155 & 15.48 & 47.14 & 28 & 6 & .12 & .9 & 1.1 & \text { DEP } \\ 2008 \text { JUN } & 8 & 0525 & 57.63 & 19 & 19.85 & 155 & 8.40 & 8.26 & 38 & 9 & .09 & .4 & .6 & \text { SF } 4\end{array}$

2008 JUN $8 \begin{array}{llllllllllllll}0538 & 49.95 & 19 & 26.53 & 155 & 15.50 & 48.77 & 44 & 9 & .10 & .7 & 1.0 & \text { DEP }\end{array}$

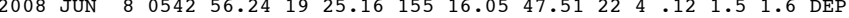

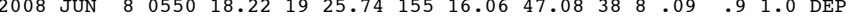
2008 JUN 80557

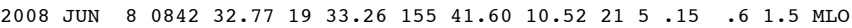
$.9 \times 31254$ $\begin{array}{rrr}1.1 \times & 288 & 36 \\ 1.8 \times & 189 & 8\end{array}$ $\begin{array}{rrr}1.8 \times & 189 & 8 \\ 1.5 \times & 252 & 18\end{array}$ $.7 \times 28445$ $.1 \times 2003$ $\begin{array}{lrl}1.8 \times & 211 & 14 \\ 1.28 & 62 & 7\end{array}$

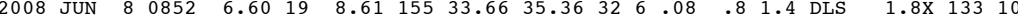

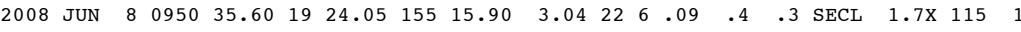

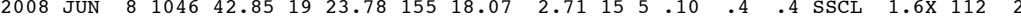

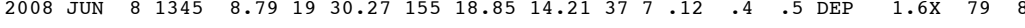

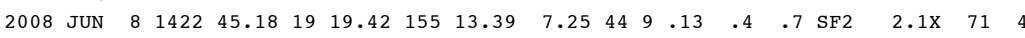

---ORIGIN TIME (HST)-- -LAT N-- --LON W-- DEPTH N N RMS ERH ERZ LOC PREF AZ MIN YEAR MON DA HRMN SEC DEG MIN DEG MIN KM RD S SEC KM KM REMKS MAG GAP DS

2008 JUN $8 \begin{array}{llllllllllllllllll}1606 & 3.89 & 19 & 18 & 85 & 155 & 13.27 & 3.83 & 28 & 4 & .11 & 3 & 1.0 & \text { SSF } & 1.5 \times & 81 & 3\end{array}$

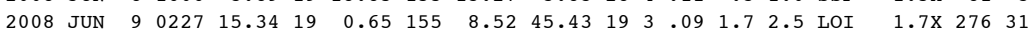

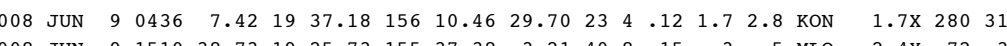
$\begin{array}{llllllllllllllll}1510 & 38.73 & 19 & 25.73 & 155 & 37.38 & 3.21 & 40 & 8 & .15 & .3 & .5 & \text { MLO } & 2.4 \mathrm{X} & 72\end{array}$

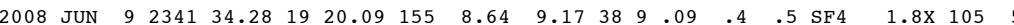

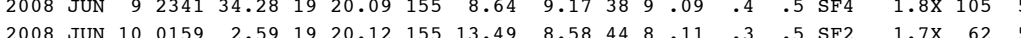

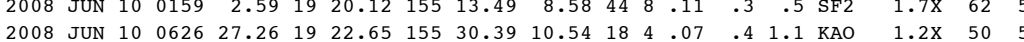

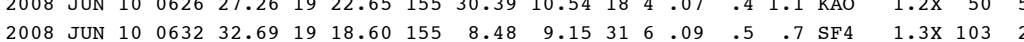

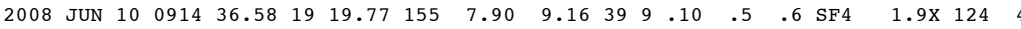

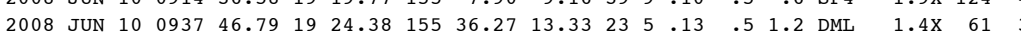

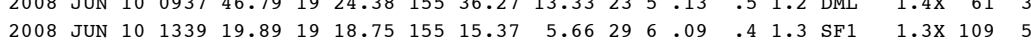

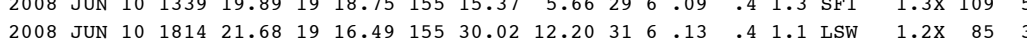

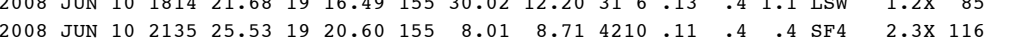

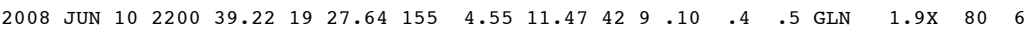

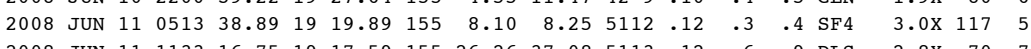

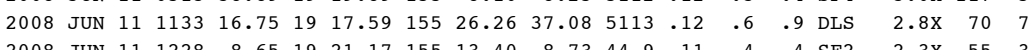

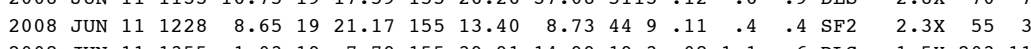

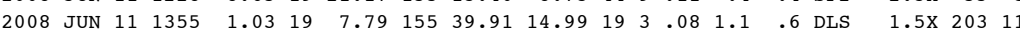

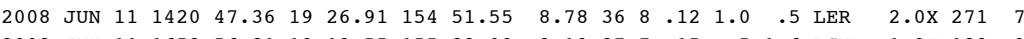

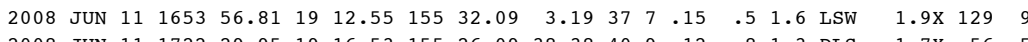

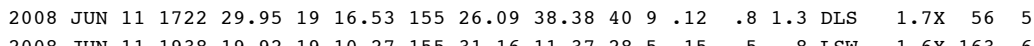

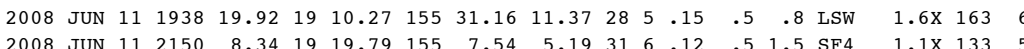

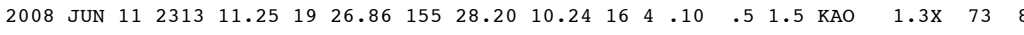

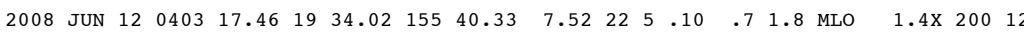

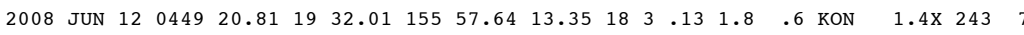

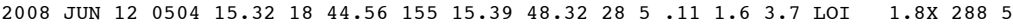

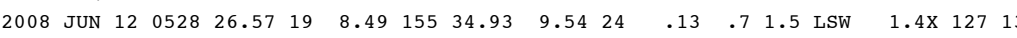

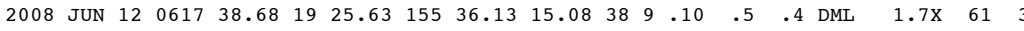

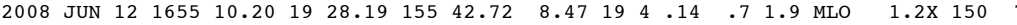

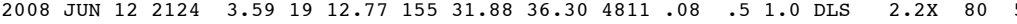

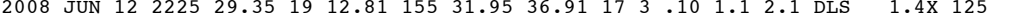

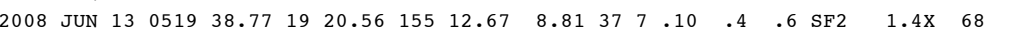

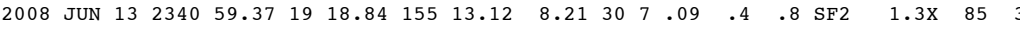

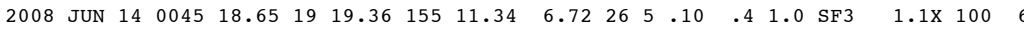

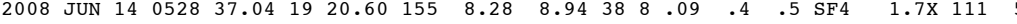

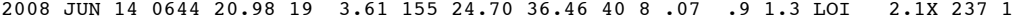

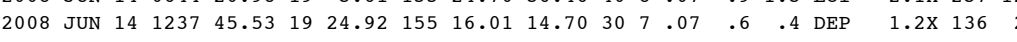

$\begin{array}{llllllllllllllllll}2008 & \text { JUN } 14 & 1243 & 27.82 & 19 & 30.68 & 155 & 22.78 & 3.23 & 28 & 8 & .14 & .5 & .3 & \text { MLO } & 1.5 \times & 156 & 2\end{array}$

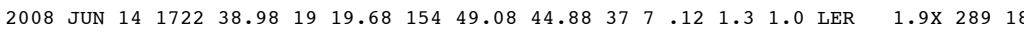

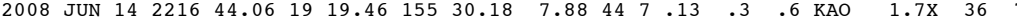

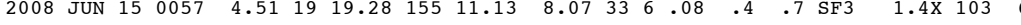

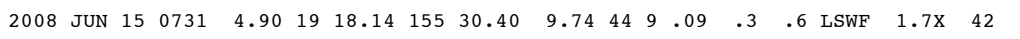


---ORIGIN TIME (HST)-- -LAT N-- --LON W-- DEPTH N N RMS ERH ERZ LOC PREF AZ MIN YEAR MON DA HRMN SEC DEG MIN DEG MIN KM RD S SEC KM KM REMKS MAG GAP DS

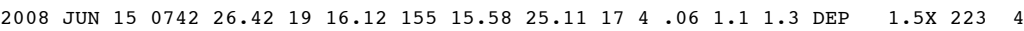

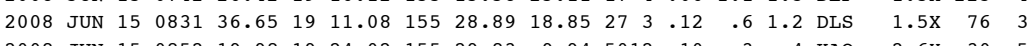
$\begin{array}{llllllllllll}2008 & & \end{array}$

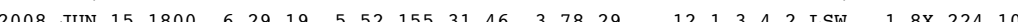

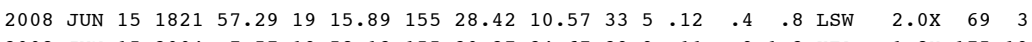

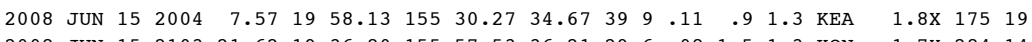

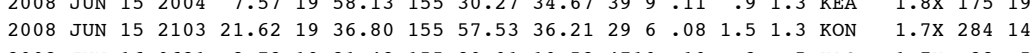

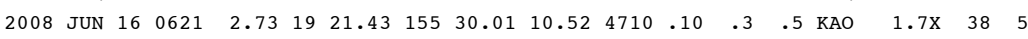

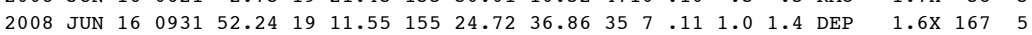

$\begin{array}{lllllllllllllllll}2008 \text { JUN } 16 & 0952 & 14.44 & 19 & 25.45 & 155 & 30.28 & 10.14 & 31 & 6 & .11 & .4 & 1.1 & \text { KAO } & 1.5 \mathrm{X} & 83 & 7\end{array}$

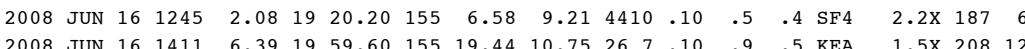

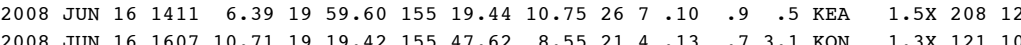

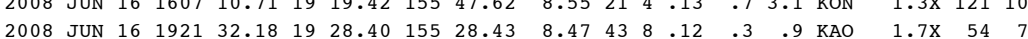

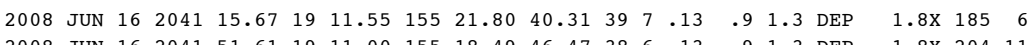

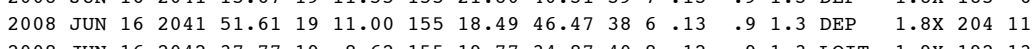

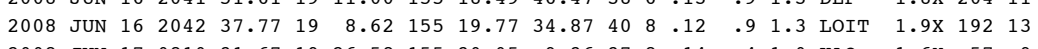

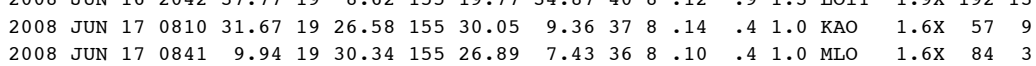

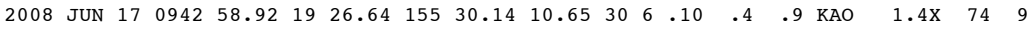

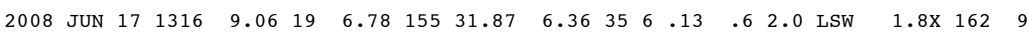

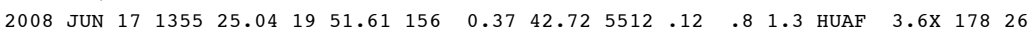

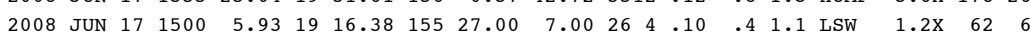

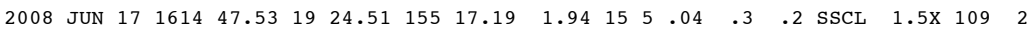

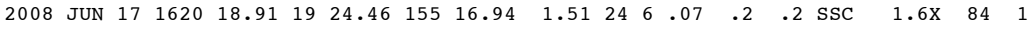

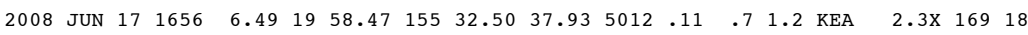
$\begin{array}{lllllllllllllllll}2008 & \text { JUN } 17 & 2133 & 7.69 & 19 & 19.75 & 155 & 8.08 & 8.51 & 4110 & .09 & .4 & .5 & \text { SF4 } & 1.8 \times & 119 & 4\end{array}$

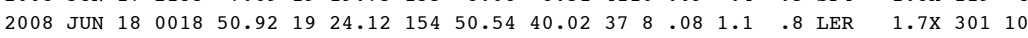

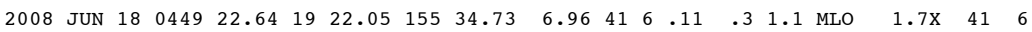

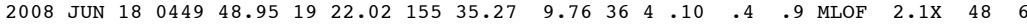

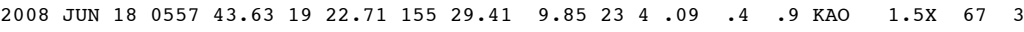

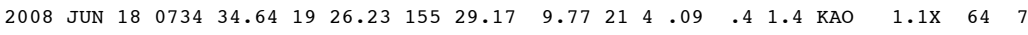

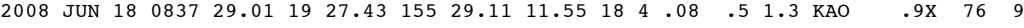

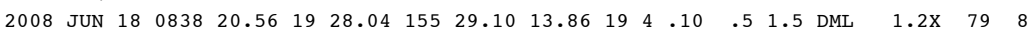

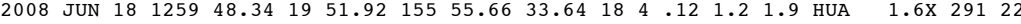

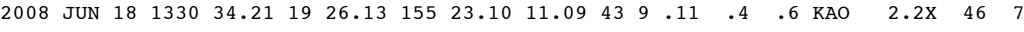

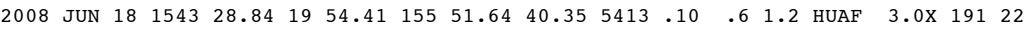

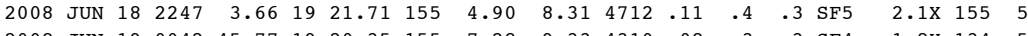

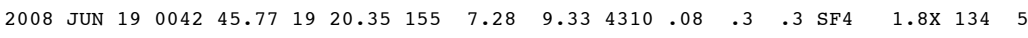

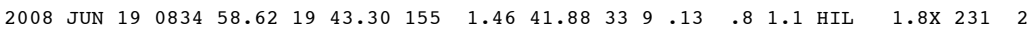

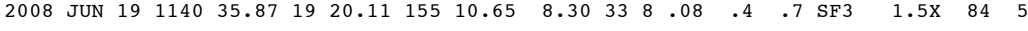

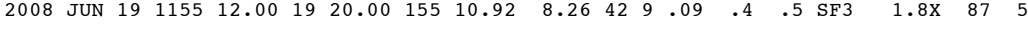

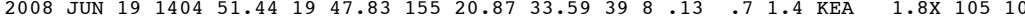

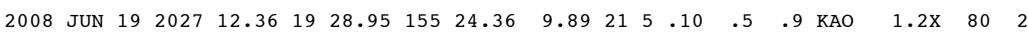

---ORIGIN TIME (HST)-- -LAT N-- --LON W-- DEPTH N N RMS ERH ERZ LOC PREF AZ MIN YEAR MON DA HRMN SEC DEG MIN DEG MIN KM RD S SEC KM KM REMKS MAG GAP DS

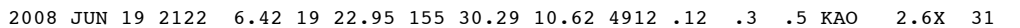

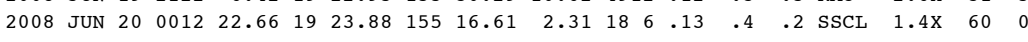

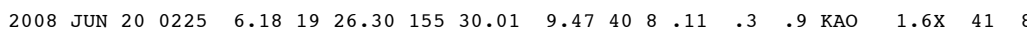

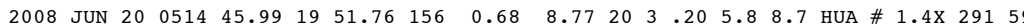

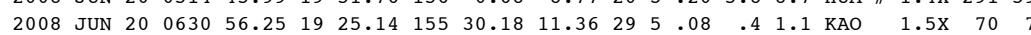

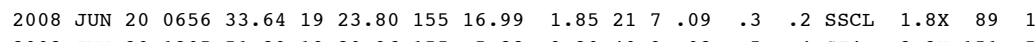

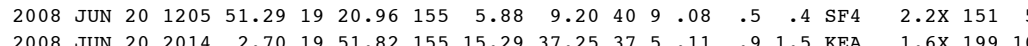

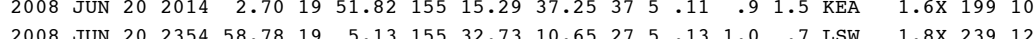

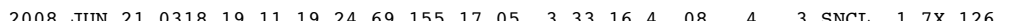

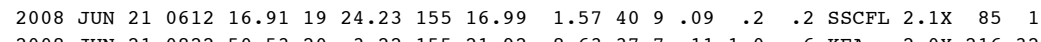

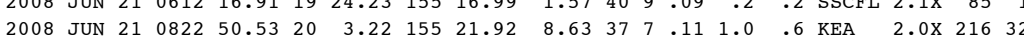

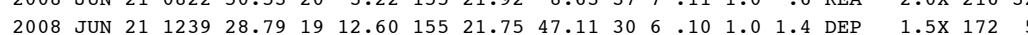
2008 JUN $21 \quad 1323 \quad 34.8418 \quad \begin{array}{lllllllllllllllll} & 51.09 & 155 & 17.15 & 47.76 & 32 & 9 & .09 & 1.1 & 1.6 & \text { LOI } & 1.7 \times & 263 & 39\end{array}$

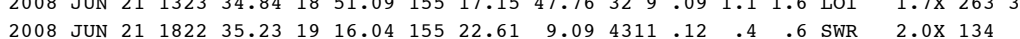

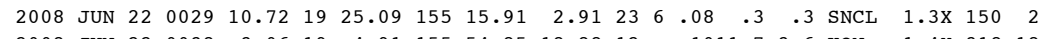

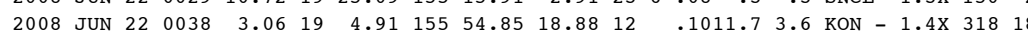

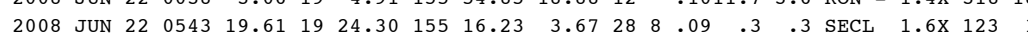

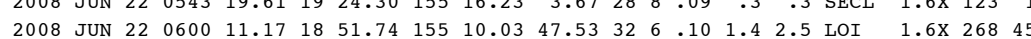

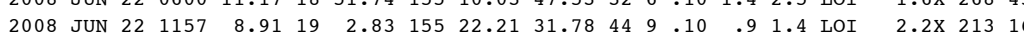

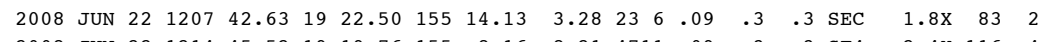

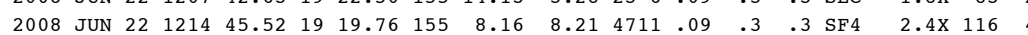

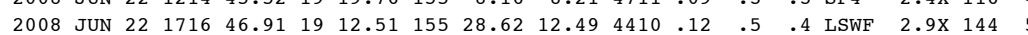

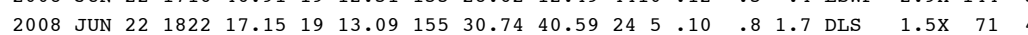

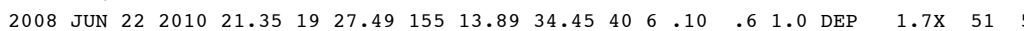

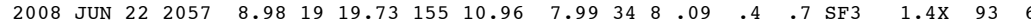

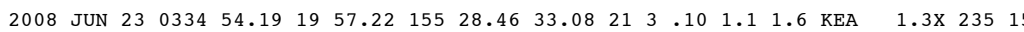

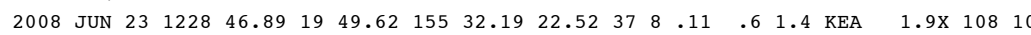

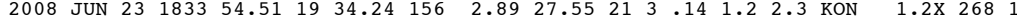

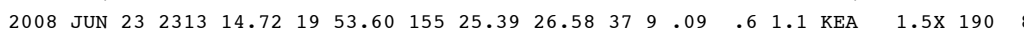

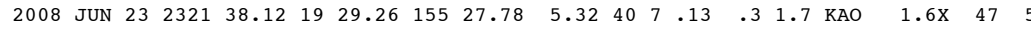

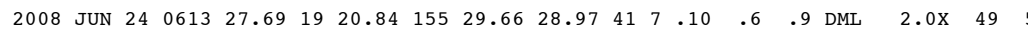

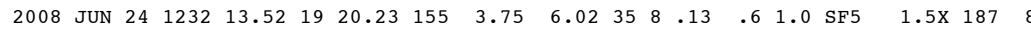

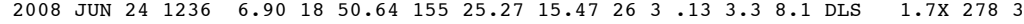

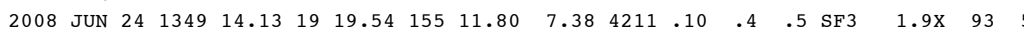

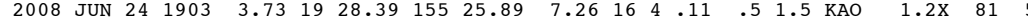

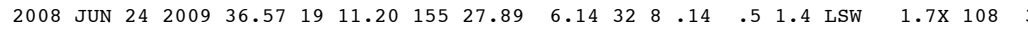

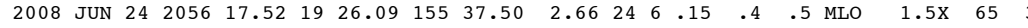

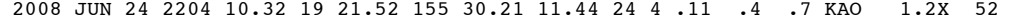

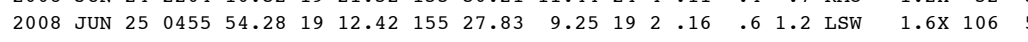

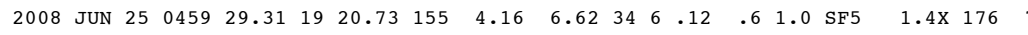

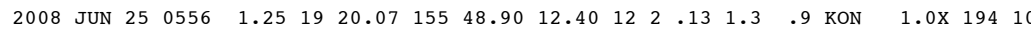

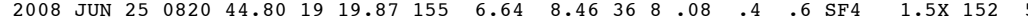

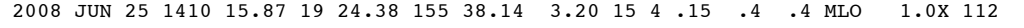

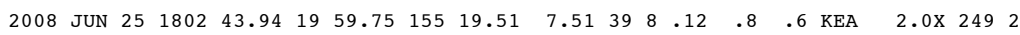


---ORIGIN TIME (HST)-- -LAT N-- --LON W-- DEPTH N N RMS ERH ERZ LOC PREF AZ MIN YEAR MON DA HRMN SEC DEG MIN DEG MIN KM RD S SEC KM KM REMKS MAG GAP DS

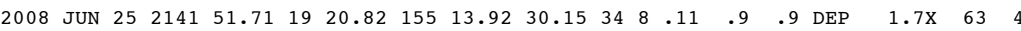

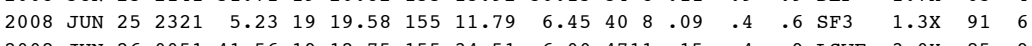

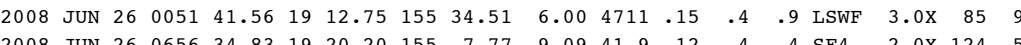

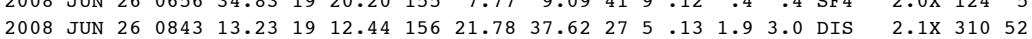

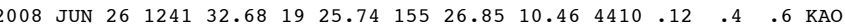

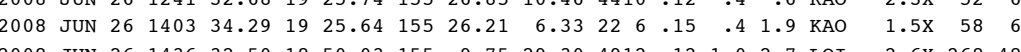

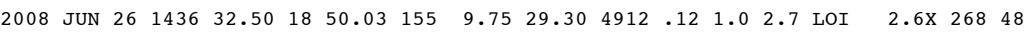

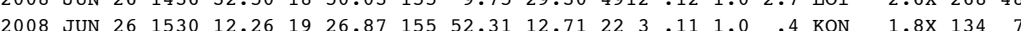

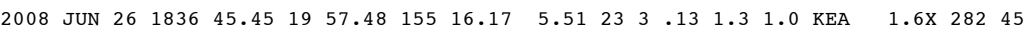

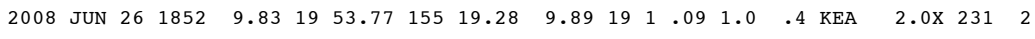

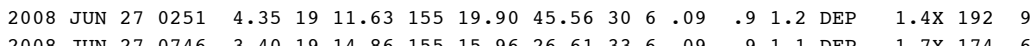

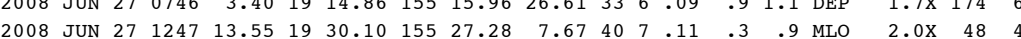

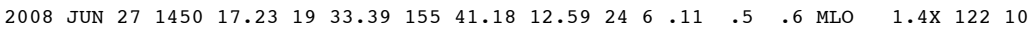

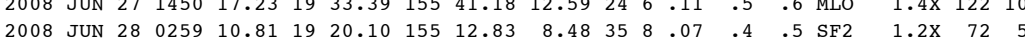

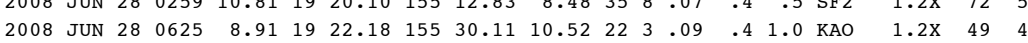

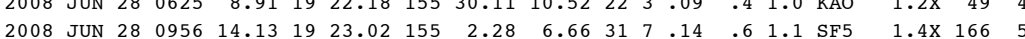

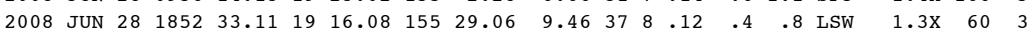

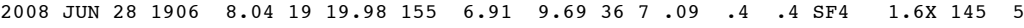

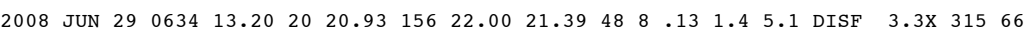

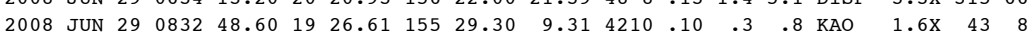

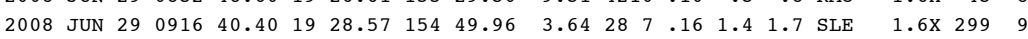

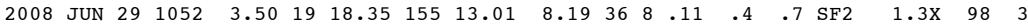

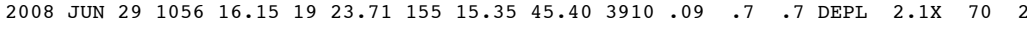

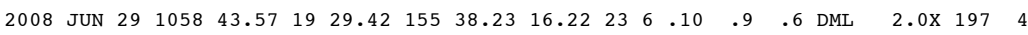

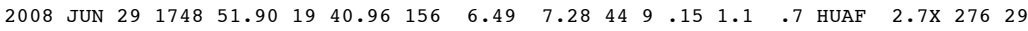

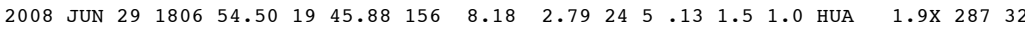

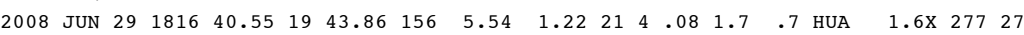

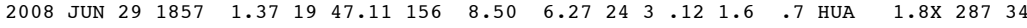

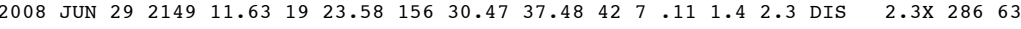

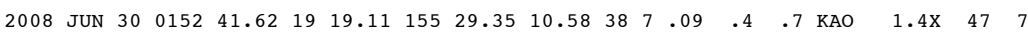

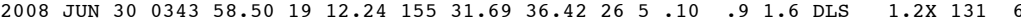

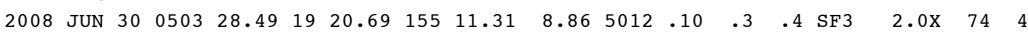

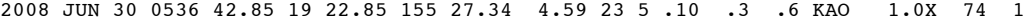

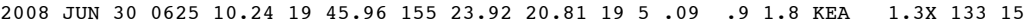

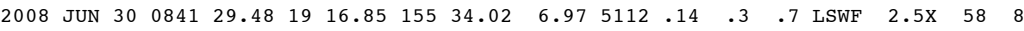

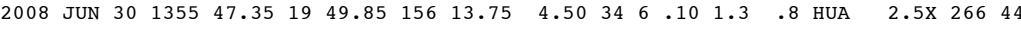

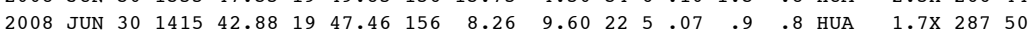

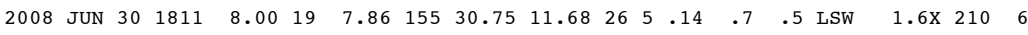
2008 JUN $30 \begin{array}{llllllllllllllll}2006 & 31.09 & 19 & 18.84 & 155 & 9.31 & 10.25 & 39 & 8 & .11 & .5 & .5 & \text { SF3 } & 1.7 \times & 107 & 4\end{array}$

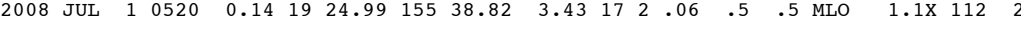

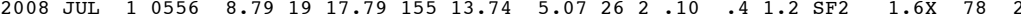

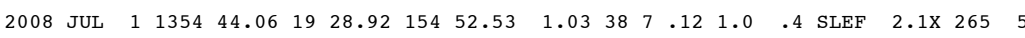

---ORIGIN TTME (HST)-- -LAT N-- --LON W-- DEPTH N N RMS ERH ERZ LOC PREF AZ MIN YEAR MON DA HRMN SEC DEG MIN DEG MIN KM RD S SEC KM KM REMKS MAG GAP DS

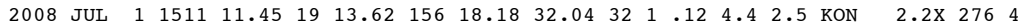

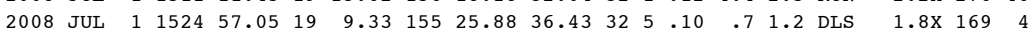

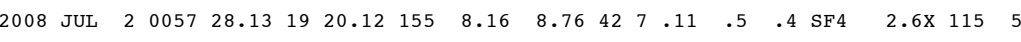
$\begin{array}{llllllllllllllllll}2008 \text { JUL } & 2 & 0121 & 48.60 & 19 & 22.04 & 155 & 0.62 & 2.01 & 24 & 1 & .13 & 1.0 & 2.3 & \text { SSF } & 1.6 \times & 194\end{array}$

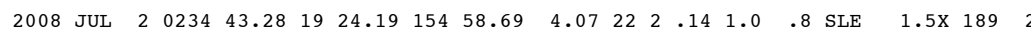

2008 JUL $20823 \quad 34.7320 \quad 54.59154 \quad 52.88 \quad 18.87 \quad 18 \quad 2.112 .712 .1$ DIS $-2.8 \times 321123$

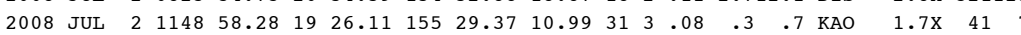

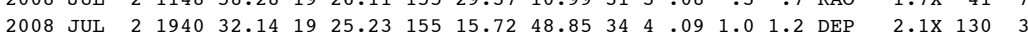

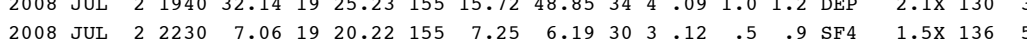
$\begin{array}{lllllllllll}2008 & 0 & 0\end{array}$

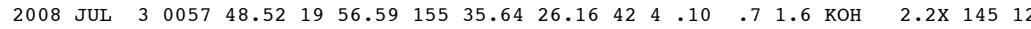
$\begin{array}{lllllllllllllllll}2008 & & & \end{array}$

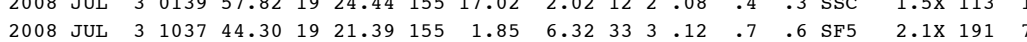
2008 JUL $3 \begin{array}{llllllllllllllll}3 & 1409 & 21.87 & 19 & 26.59 & 155 & 29.53 & 10.91 & 21 & 3 & .07 & .4 & 1.2 & \text { KAO } & 1.6 \mathrm{X} & 68\end{array}$

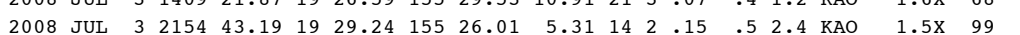

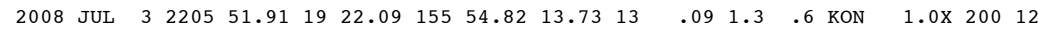

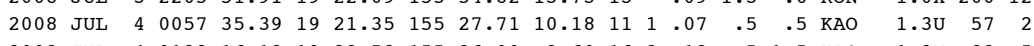

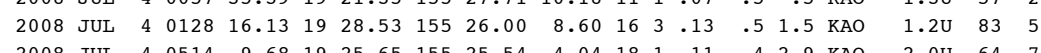

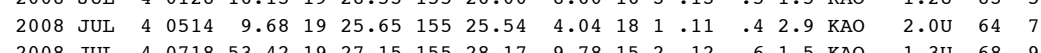

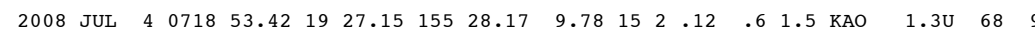

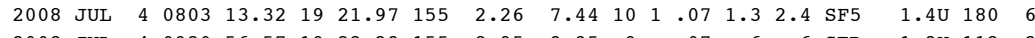

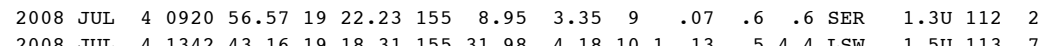

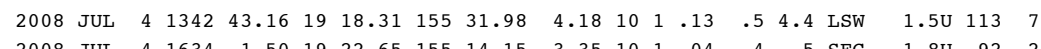

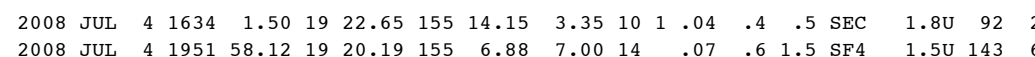

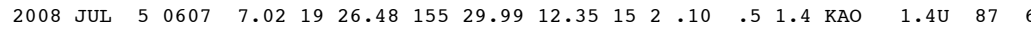
$\begin{array}{llllllllllllllllll}2008 & \text { JUL } & 5 & 1150 & 12.84 & 19 & 22.25 & 155 & 27.24 & 8.65 & 17 & 1 & .14 & .7 & 1.0 & \text { KAO } & 1.7 \mathrm{U} & 76\end{array}$

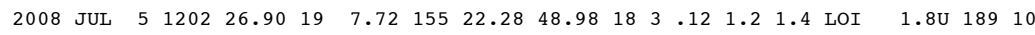
2008 JUL $5 \begin{array}{lllllllllllllll}2244 & 56.23 & 19 & 25.65 & 155 & 37.97 & 3.30 & 12 & 3 & .08 & 1.0 & .5 & \text { MLO } & 2.0 U & 182\end{array}$

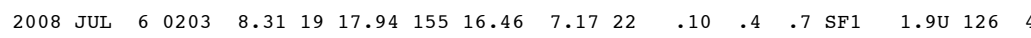

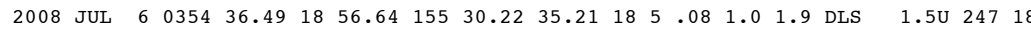

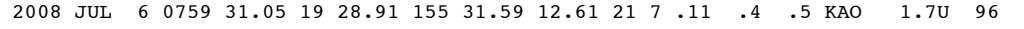

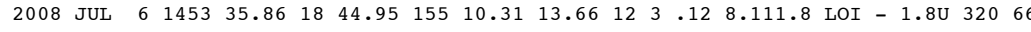
2008 JUL $6 \begin{array}{llllllllllllllll}6 & 1925 & 38.98 & 19 & 23.24 & 155 & 15.27 & 29.83 & 18 & 1 & .10 & 1.2 & 2.3 & \text { DEP } & 1.5 U & 128\end{array}$ 2008 JUL $7 \begin{array}{llllllllllllllll}0302 & 17.28 & 19 & 16.01 & 155 & 27.71 & 9.59 & 13 & 1 & .10 & .5 & .8 & \text { LSW } & 1.8 U & 133\end{array}$

2008 JUL $7 \begin{array}{lllllllllllllllll} & 0419 & 44.42 & 19 & 27.45 & 155 & 25.07 & 2.54 & 13 & 2 & .11 & .5 & 1.2 & \text { KAO } & 1.3 \mathrm{U} & 80 & 5\end{array}$

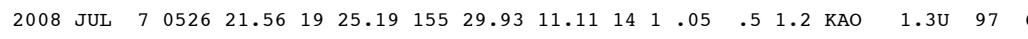

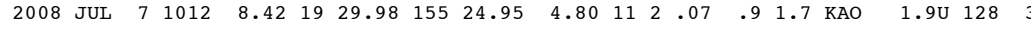
2008 JUL $7 \begin{array}{lllllllllllllll}1351 & 21.34 & 19 & 20.66 & 155 & 11.29 & 7.84 & 25 & 3 & .10 & .6 & .8 & \mathrm{SF} 3 & 1.6 \mathrm{X} & 84\end{array}$

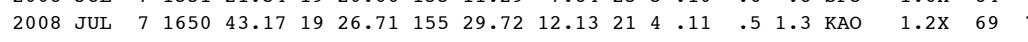

$\begin{array}{lllllllllllllllllll}2008 & \text { JUL } & 7 & 1730 & 57.97 & 19 & 24.41 & 155 & 16.93 & 1.59 & 25 & 6 & .09 & .3 & .2 & \text { SSC } & 2.2 \times & 115 & 1\end{array}$ $\begin{array}{lllllllllllllllllll}2008 & \text { JUL } & 7 & 1850 & 32.54 & 19 & 29.46 & 155 & 26.72 & 7.37 & 33 & 4 & .12 & .4 & 1.0 & \mathrm{KAO} & 1.8 \mathrm{x} & 70 & 5\end{array}$

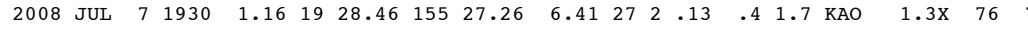
2008 JUL $7 \begin{array}{llllllllllllllll}2046 & 57.40 & 19 & 24.85 & 155 & 26.28 & 1.77 & 20 & 2 & .12 & .3 & 1.5 & \text { KAO } & 1.4 \mathrm{X} & 57 & 5\end{array}$

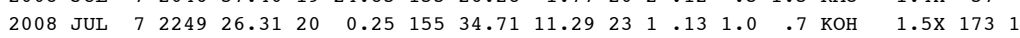


---ORIGIN TIME (HST)-- -LAT N-- --LON W-- DEPTH N N RMS ERH ERZ LOC PREF AZ MIN YEAR MON DA HRMN SEC DEG MIN DEG MIN KM RD S SEC KM KM REMKS MAG GAP DS

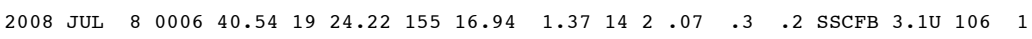

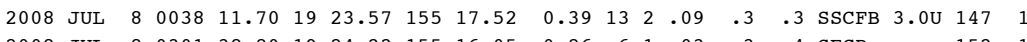

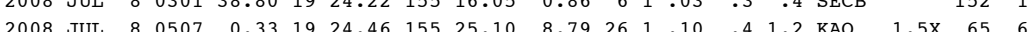

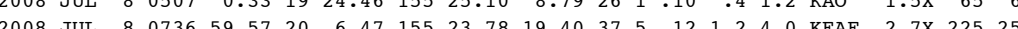

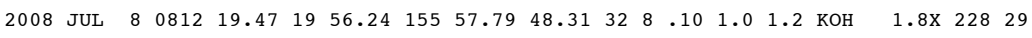

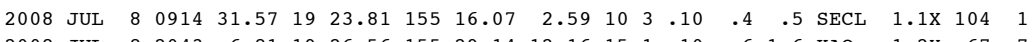
$\begin{array}{llllllllllllllllllll}2008 & \text { JUL } & 8 & 2043 & 6.81 & 19 & 26.56 & 155 & 29.14 & 12.16 & 15 & 1 & .10 & .6 & 1.6 & \text { KAO } & 1.2 \mathrm{X} & 67 & 7 \\ 2008 & & \end{array}$

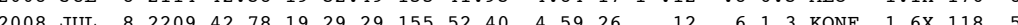

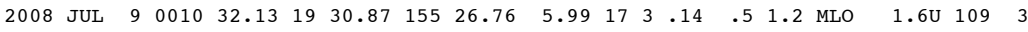

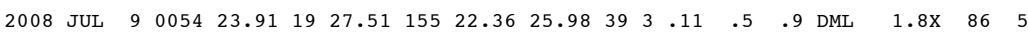

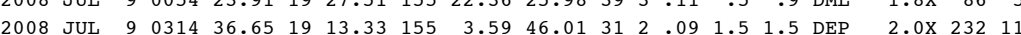
2008 JUL $991940 \begin{array}{llllllllllllllll}2.09 & 19 & 27.17 & 155 & 26.04 & 1.75 & 17 & 3 & .11 & .4 & 1.2 & \text { KAO } & 1.0 x & 61 & 7\end{array}$

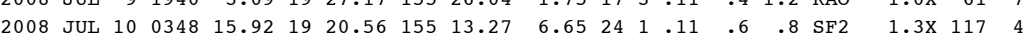

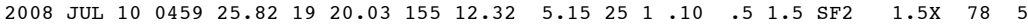

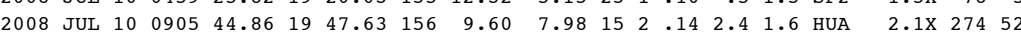

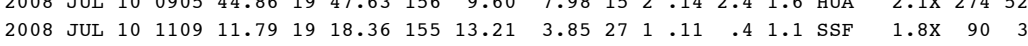

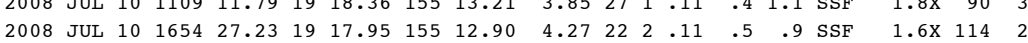

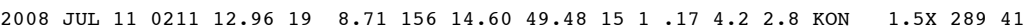

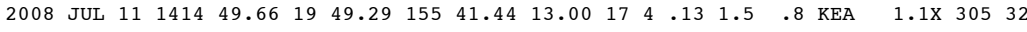

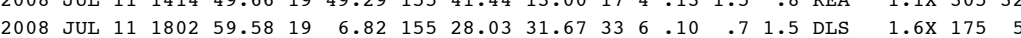

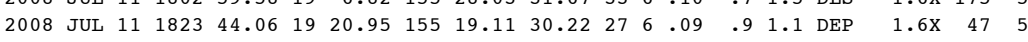

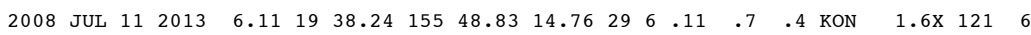

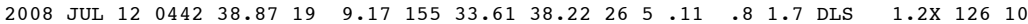

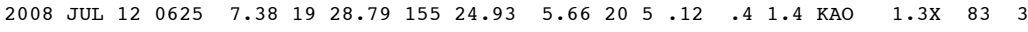

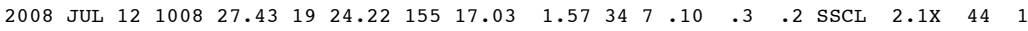

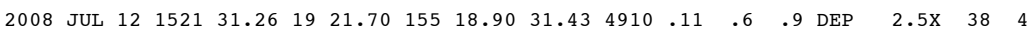

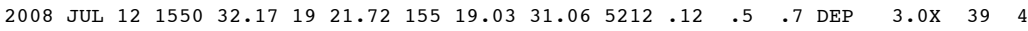

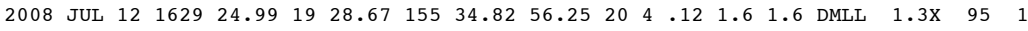

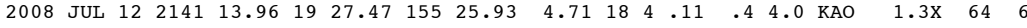

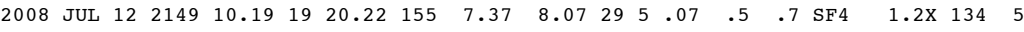

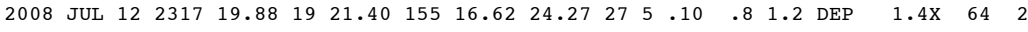

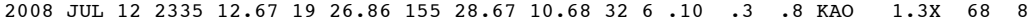

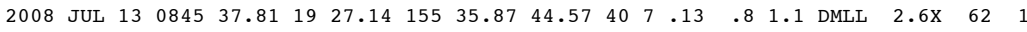

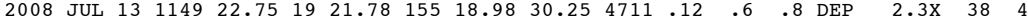

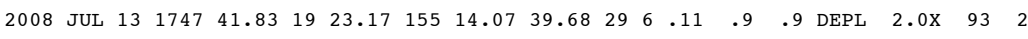

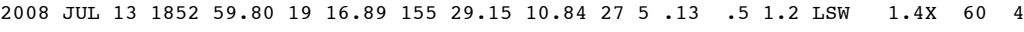

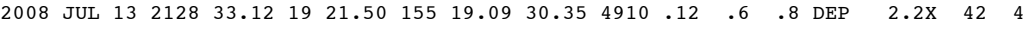

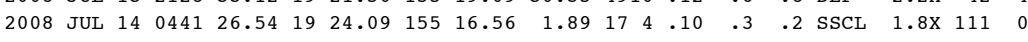

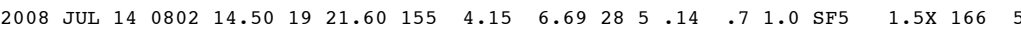

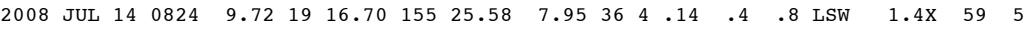

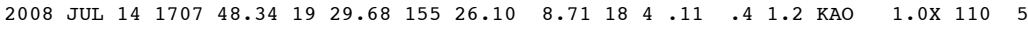

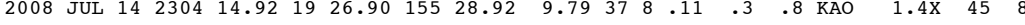

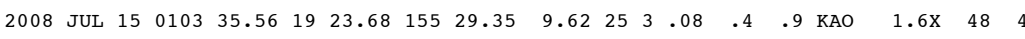

---ORIGIN TIME (HST)-- -LAT N-- --LON W-- DEPTH N N RMS ERH ERZ LOC PREF AZ MIN YEAR MON DA HRMN SEC DEG MIN DEG MIN KM RD S SEC KM KM REMKS MAG GAP DS 2008 JUL $15 \quad \begin{array}{lllllllllllllllll}15 & 0503 & 46.13 & 19 & 19.28 & 155 & 11.59 & 7.51 & 33 & 7 & 09 & .4 & 9 & \text { SF } 3 & 1.4 X & 100 & -5\end{array}$

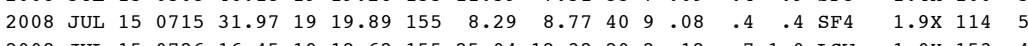

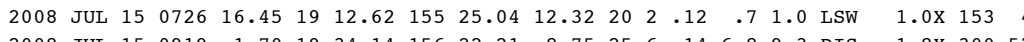
$\begin{array}{lllllllllllll}2008 & & & \end{array}$

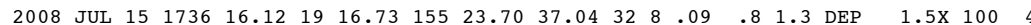

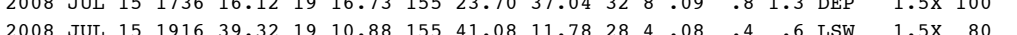

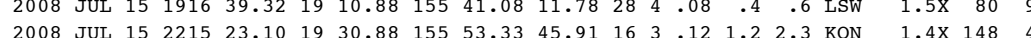

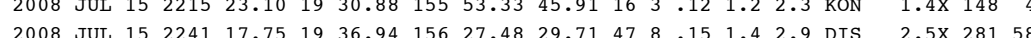

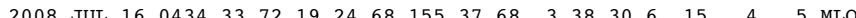

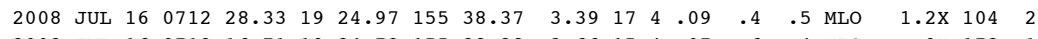

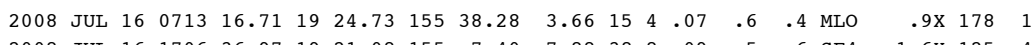

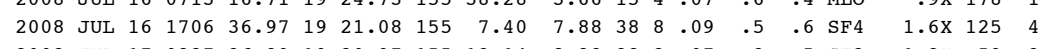

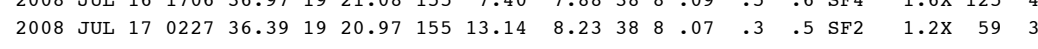

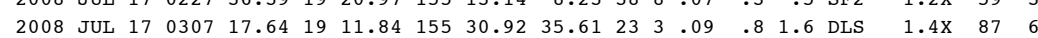

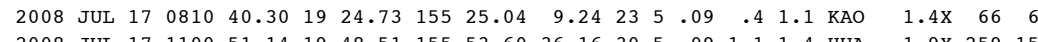

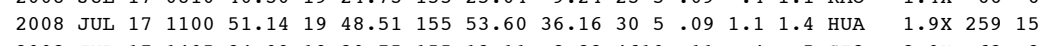
$\begin{array}{lllllllllllllllll}2008 & \text { JUL } 17 & 1405 & 34.09 & 19 & 20.75 & 155 & 13.11 & 8.82 & 4610 & .11 & .4 & .5 & \text { SF2 } & 2.0 \mathrm{x} & 62 & 3\end{array}$

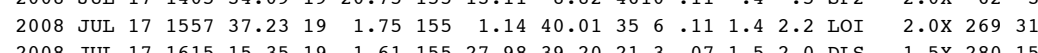

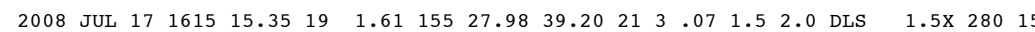

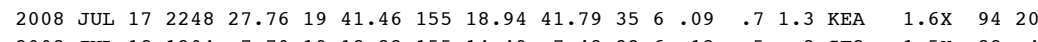

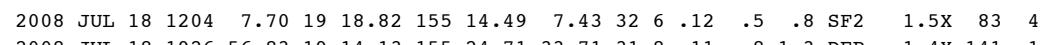

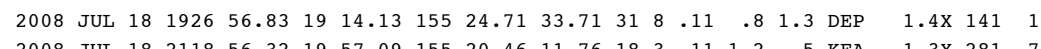

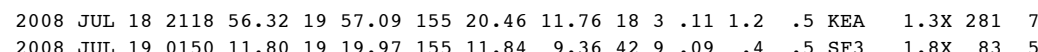

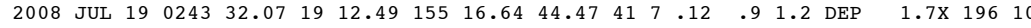

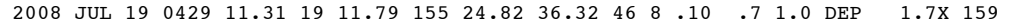

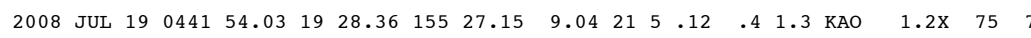

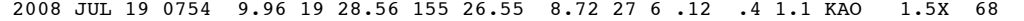

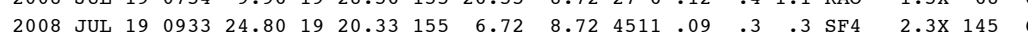

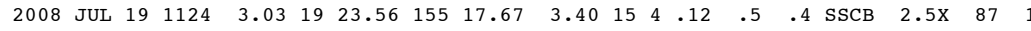

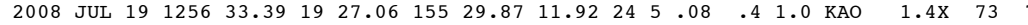

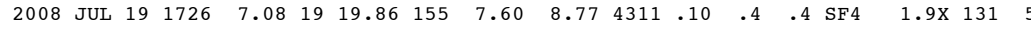

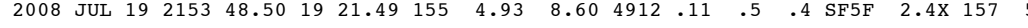

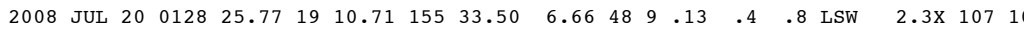

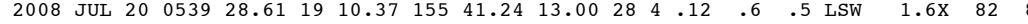

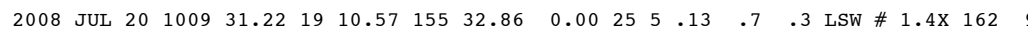

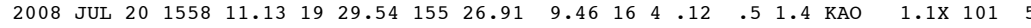

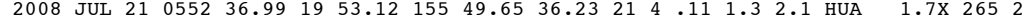

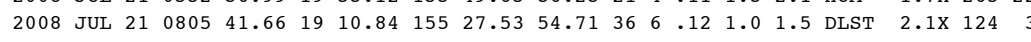

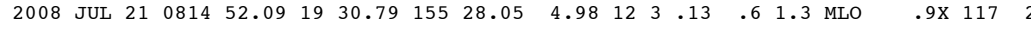

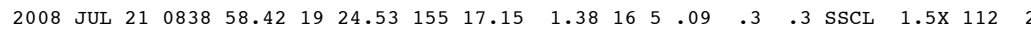

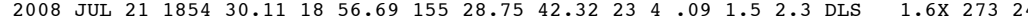

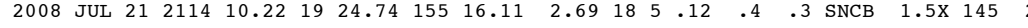

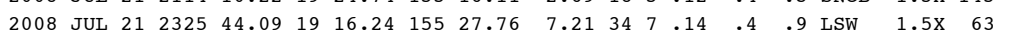


---ORIGIN TIME (HST)-- -LAT N-- --LON W-- DEPTH N N RMS ERH ERZ LOC PREF AZ MIN YEAR MON DA HRMN SEC DEG MIN DEG MIN KM RD S SEC KM KM REMKS MAG GAP DS

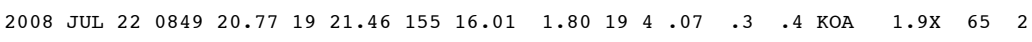

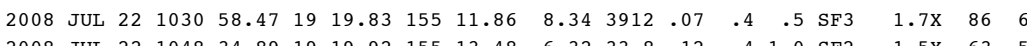

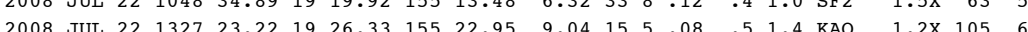
$\begin{array}{llllllllllllll}2008 & & \end{array}$

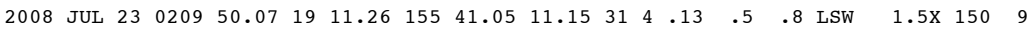

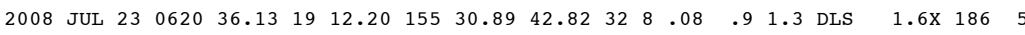

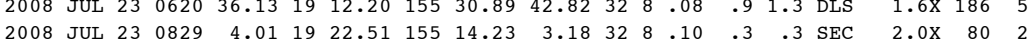

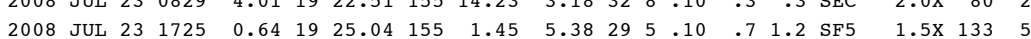

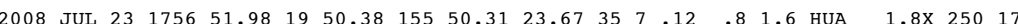

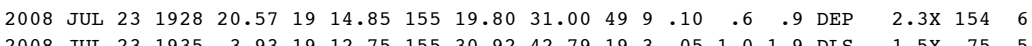

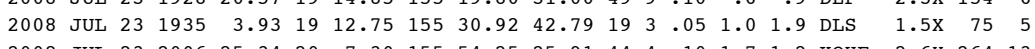

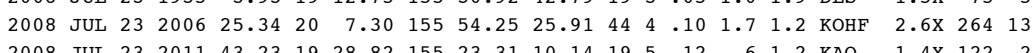

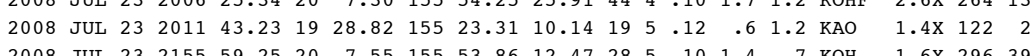

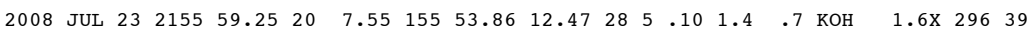

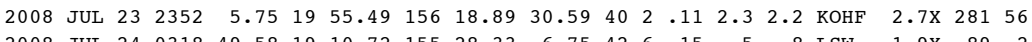
2008 JUL $24 \quad \begin{array}{lllllllllllllllll}0318 & 49.58 & 19 & 10.72 & 155 & 28.33 & 6.75 & 42 & 6 & .15 & .5 & .8 & \text { LSW } & 1.9 \times & 89 & 2\end{array}$

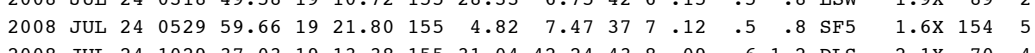

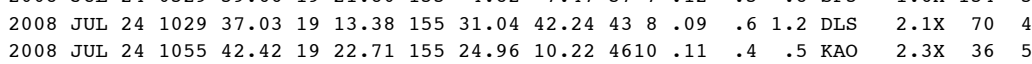

$\begin{array}{lllllllllllllllll}2008 & \text { JUL } 24 & 1205 & 48.23 & 19 & 20.53 & 155 & 11.03 & 7.68 & 4210 & .11 & .4 & .6 & \text { SF3 } & 2.1 \times & 77 & 4\end{array}$

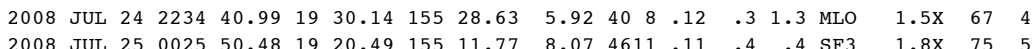

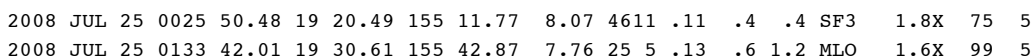

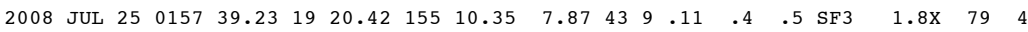

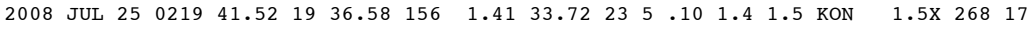

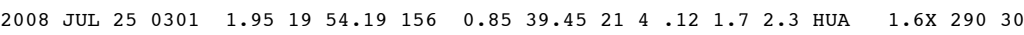

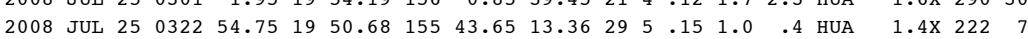

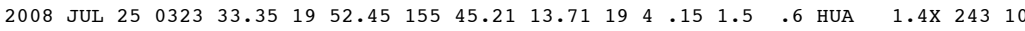

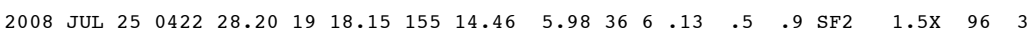

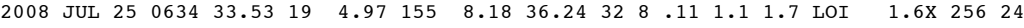

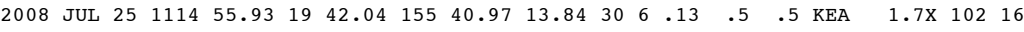

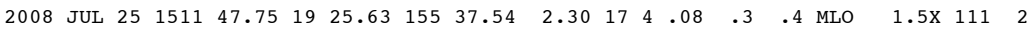

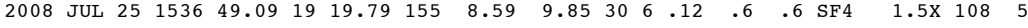

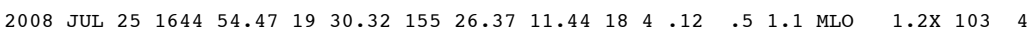

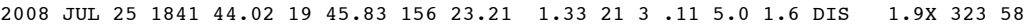

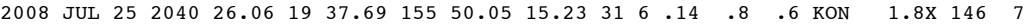

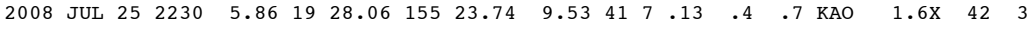

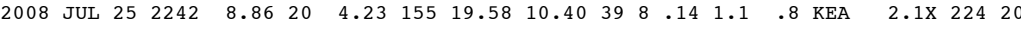

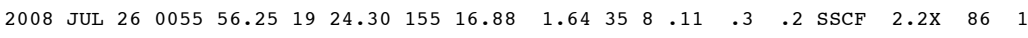

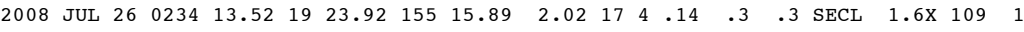

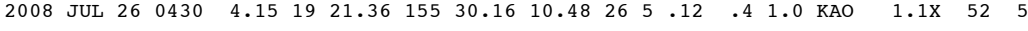

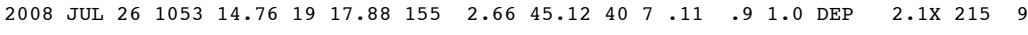

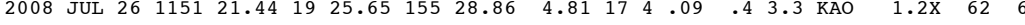

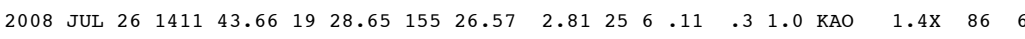

---ORIGIN TTME (HST)-- -LAT N-- --LON W-- DEPTH N N RMS ERH ERZ LOC PREF AZ MIN YEAR MON DA HRMN SEC DEG MIN DEG MIN KM RD S SEC KM KM REMKS MAG GAP DS

2008 JUL $26 \quad \begin{array}{lllllllllllllllll}1505 & 19.02 & 19 & 29.15 & 155 & 27.99 & 8.71 & 20 & 5 & .09 & 4 & 1.3 & \text { KAO } & 1.1 X & 80 & -5\end{array}$

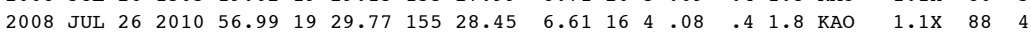

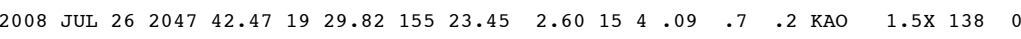

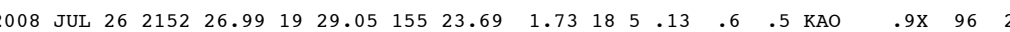

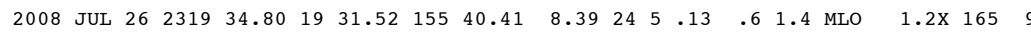

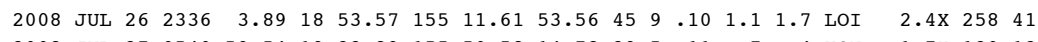

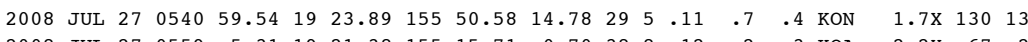

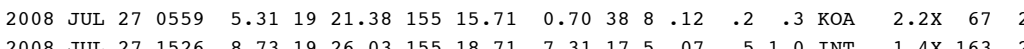

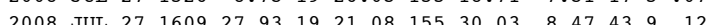

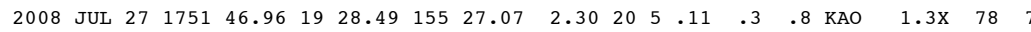

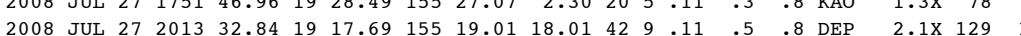

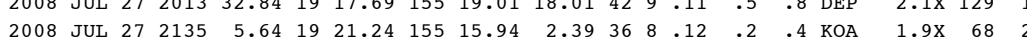

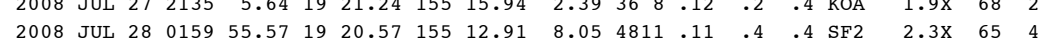

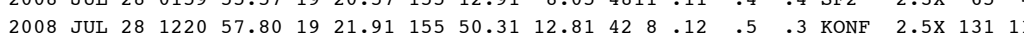

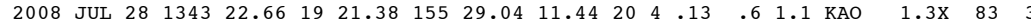

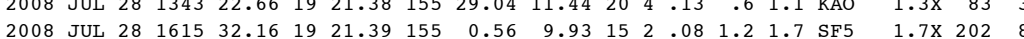
2008 JUL $290045 \quad 12.3619 \quad 48.49155 \quad 24.47 \begin{array}{llllllllll}17.76 & 36 & 8 & .10 & .6 & 1.5 & \text { KEA } & 1.6 \times & 142\end{array}$

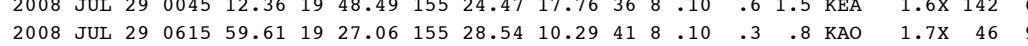

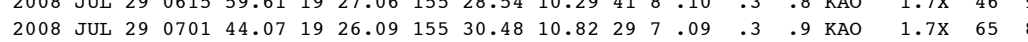

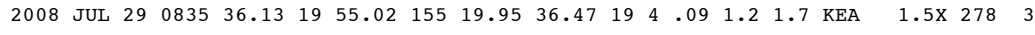

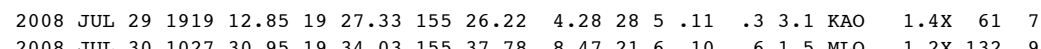

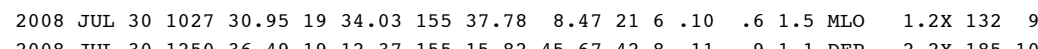

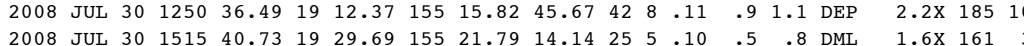

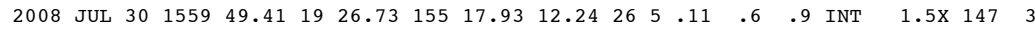

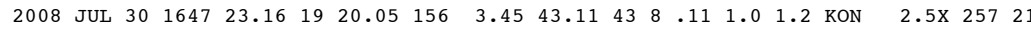

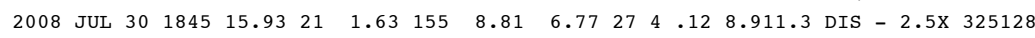

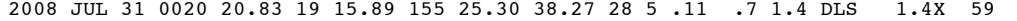

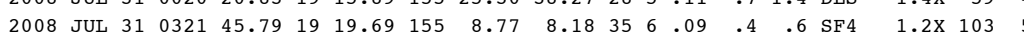

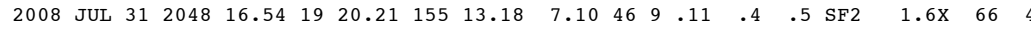

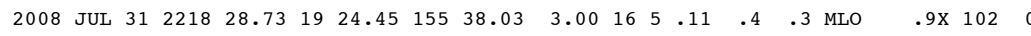

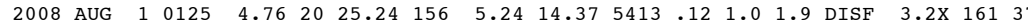
2008 AUG $110419 \begin{array}{lllllllllllllll}36.44 & 19 & 20.05 & 155 & 8.82 & 8.33 & 43 & 9 & .10 & .4 & .4 & \mathrm{SF} 4 & 2.0 \mathrm{X} & 101\end{array}$

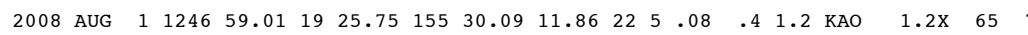

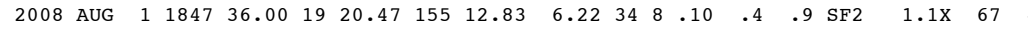

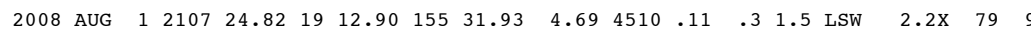
2008 AUG $12109 \begin{array}{llllllllllllll}25.81 & 19 & 24.46 & 155 & 17.08 & 0.70 & 18 & 5 & .10 & .2 & .2 & \text { SSCB } & 2.5 \times & 112\end{array}$

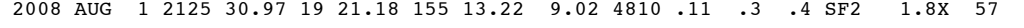

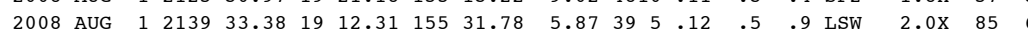

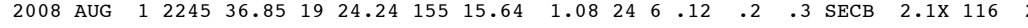

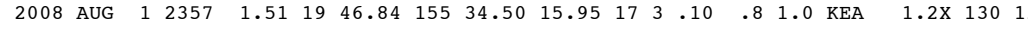

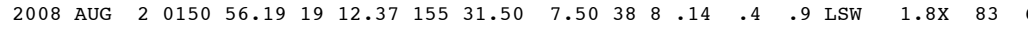

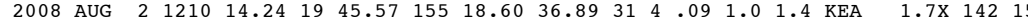

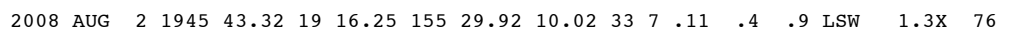


---ORIGIN TIME (HST)-- -LAT N-- --LON W-- DEPTH N N RMS ERH ERZ LOC PREF AZ MIN YEAR MON DA HRMN SEC DEG MIN DEG MIN KM RD S SEC KM KM REMKS MAG GAP DS

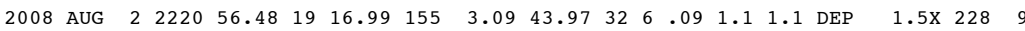

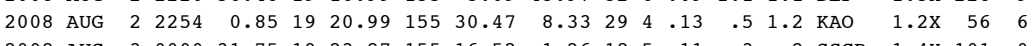

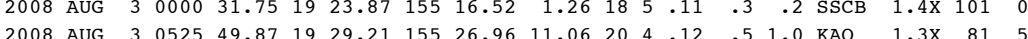

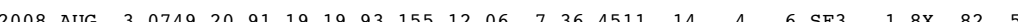

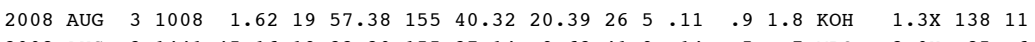
2008 AUG $3 \begin{array}{lllllllllllllllll}3 & 1441 & 45.16 & 19 & 32.20 & 155 & 37.14 & 9.63 & 41 & 9 & .14 & .5 & .7 & \text { MLO } & 2.0 \mathrm{X} & 85 & 6\end{array}$

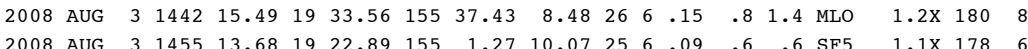

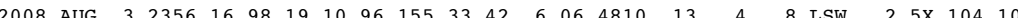

$\begin{array}{lllllllllllllllllll}2008 & \text { AUG } & 4 & 0134 & 29.87 & 19 & 27.30 & 155 & 27.63 & 11.45 & 18 & 5 & .12 & .5 & 1.4 & \text { KAO } & 1.1 \mathrm{X} & 67 & 9\end{array}$

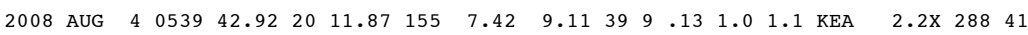

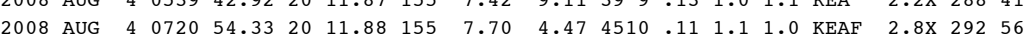

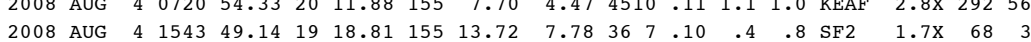

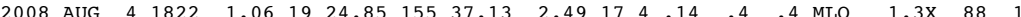
$\begin{array}{lllllllllllllllllll}2008 \text { AUG } & 5 & 0536 & 44.50 & 19 & 24.04 & 155 & 16.51 & 2.10 & 16 & 4 & .13 & .4 & .2 & \text { SSCB } & 1.4 \mathrm{X} & 109 & 0 \\ 2008 \text { AUG } & 5 & 0557 & 51.06 & 19 & 19.20 & 155 & 26.54 & 10.39 & 45 & 9 & .12 & .3 & .6 & \text { KAO } & 1.5 \mathrm{X} & 54 & 6\end{array}$ 2008 AUG $5 \begin{array}{lllllllllllllllll}5 & 0722 & 7.36 & 19 & 10.65 & 155 & 9.10 & 48.85 & 45 & 8 & .11 & 1.0 & 1.2 & \text { DEP } & 2.3 \times & 212 & 13\end{array}$ 2008 AUG $5 \begin{array}{lllllllllllllllll} & 1606 & 12.67 & 19 & 0.27 & 155 & 27.48 & 35.14 & 36 & 7 & .08 & 1.0 & 1.6 & \text { DLS } & 2.3 \times & 251 & 17\end{array}$ 2008 AUG $5 \begin{array}{lllllllllllllllll}1608 & 53.63 & 18 & 58.27 & 155 & 27.51 & 35.04 & 33 & 6 & .12 & 1.2 & 1.7 & \text { DLS } & 2.1 \times & 230 & 21\end{array}$

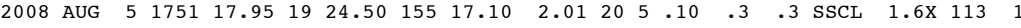
2008 AUG $5 \begin{array}{lllllllllllllll}1942 & 55.38 & 19 & 22.36 & 155 & 26.58 & 11.10 & 4410 & .11 & .4 & .5 & \text { KAO } & 1.8 \times & 39 & 2\end{array}$

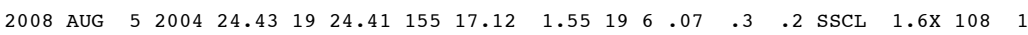

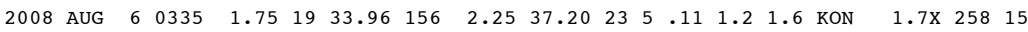

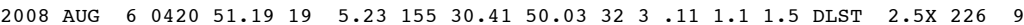

2008 AUG $6 \begin{array}{lllllllllllllllll} & 1404 & 56.52 & 19 & 24.24 & 155 & 16.95 & 1.75 & 34 & 8 & .08 & .2 & .2 & \text { SSC } & 2.2 \mathrm{X} & 85 & 1\end{array}$

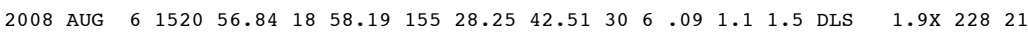
2008 AUG $6 \begin{array}{lllllllllllllllll}6 & 2304 & 49.41 & 19 & 18.39 & 155 & 27.76 & 12.00 & 21 & 4 & .11 & .4 & 1.0 & \text { LSW } & .9 x & 43 & 7\end{array}$

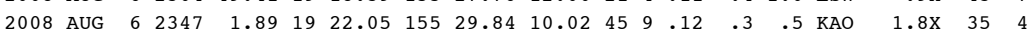

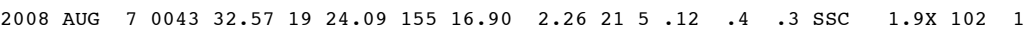

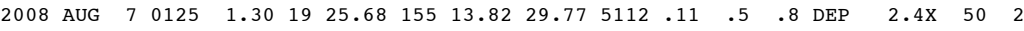

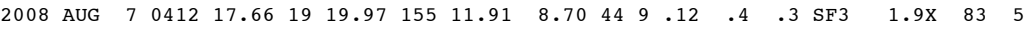

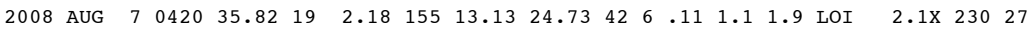
2008 AUG $7 \begin{array}{lllllllllllllllll}0506 & 38.58 & 19 & 20.02 & 155 & 7.96 & 6.90 & 29 & 8 & .10 & .4 & .9 & \text { SF4 } & 1.3 \times & 120 & 5\end{array}$

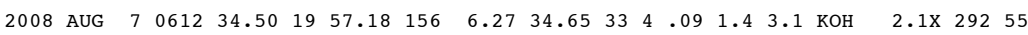

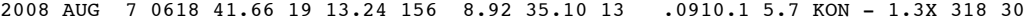
2008 AUG $7 \begin{array}{llllllllllllllll}0705 & 32.97 & 19 & 36.52 & 155 & 24.56 & 45.99 & 24 & 3 & .12 & 1.6 & 1.6 & \text { KEA } & 1.7 \times & 164 & 8\end{array}$

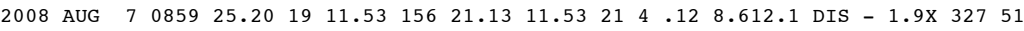

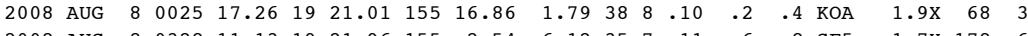

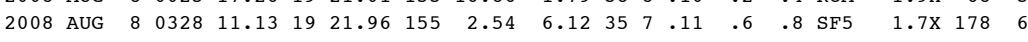

$\begin{array}{lllllllllllllllllll}2008 & \text { AUG } & 8 & 0742 & 37.59 & 19 & 25.85 & 155 & 19.33 & 7.65 & 22 & 6 & .07 & .5 & .8 & \text { KAO } & 1.8 \times & 143 & 3\end{array}$

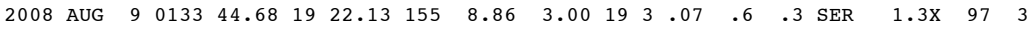

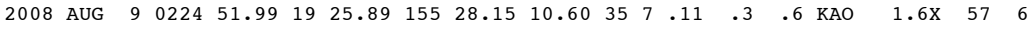

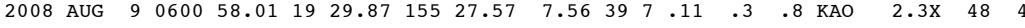
2008 AUG $9 \begin{array}{llllllllllllllll}1215 & 58.89 & 19 & 15.11 & 156 & 14.72 & 29.48 & 15 & .09 & 7.7 & 3.4 & \text { KON } & 1.6 \times & 310 & 39\end{array}$
---ORTGIN TIME (HST)-- -LAT N-- --LON W-- DEPTH N N RMS ERH ERZ LOC PREF AZ MIN YEAR MON DA HRMN SEC DEG MIN DEG MIN KM RD S SEC KM KM REMRS MAG GAP DS

2008 AUG $9 \begin{array}{llllllllllllllll}1422 & 3.29 & 19 & 2.18 & 155 & 22.60 & 35.33 & 41 & 8 & .08 & .9 & 1.3 & \text { LOI } & 2.1 \mathrm{X} & 215 & 16\end{array}$

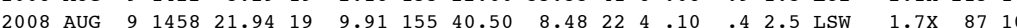

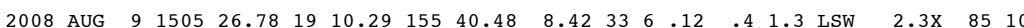

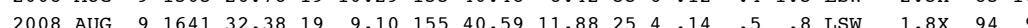

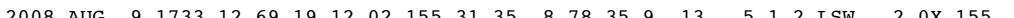

$\begin{array}{llllllllllllllllll}2008 & \text { AUG } & 9 & 2248 & 9.67 & 19 & 48.24 & 155 & 42.76 & 14.63 & 5112 & .11 & .7 & .6 & \text { KEAF } & 2.6 \times & 123\end{array}$

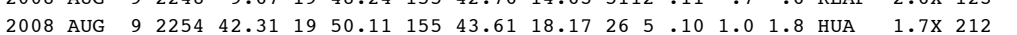

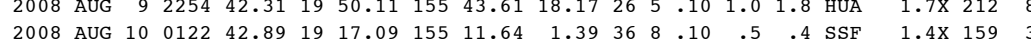

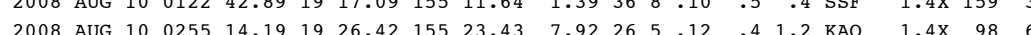

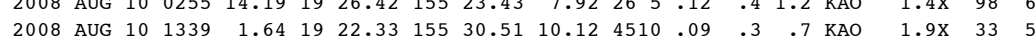

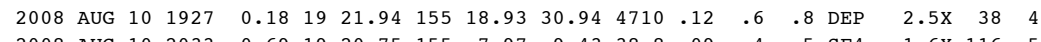

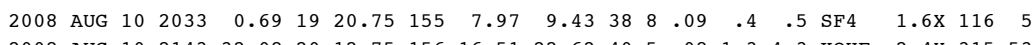

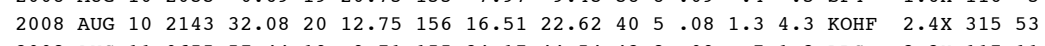

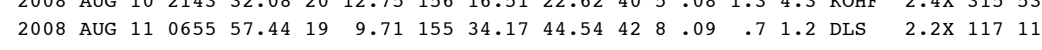

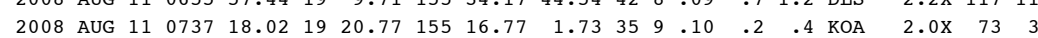

$\begin{array}{llllllllllllllllll}2008 & \text { AUG } 11 & 1029 & 18.51 & 19 & 29.73 & 155 & 26.15 & 6.92 & 18 & 4 & .08 & .4 & 1.2 & \text { KAO } & 1.6 \mathrm{X} & 76 & 5\end{array}$

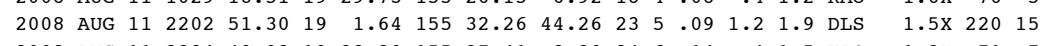
$\begin{array}{lllllllllllllllll}2008 \text { AUG } 11 & 2334 & 49.92 & 19 & 28.20 & 155 & 27.41 & 8.30 & 24 & 6 & .14 & .4 & 1.5 & \text { KAO } & 1.2 \mathrm{X} & 71 & 7\end{array}$

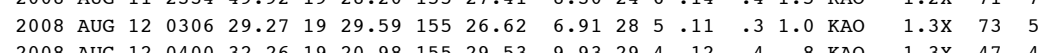

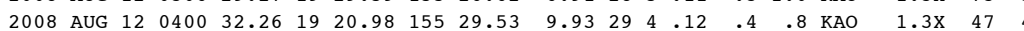

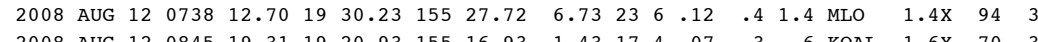
$\begin{array}{lllllllllllllllll}2008 \text { AUG } 12 & 0845 & 19.31 & 19 & 20.93 & 155 & 16.93 & 1.43 & 17 & 4 & .07 & .3 & .6 & \text { KOAL } & 1.6 \mathrm{X} & 70 & 3\end{array}$

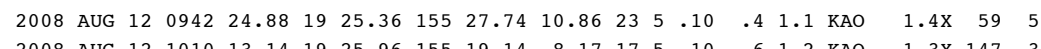
$\begin{array}{llllllllllllllllll}2008 \text { AUG } 12 & 1010 & 13.14 & 19 & 25.96 & 155 & 19.14 & 8.17 & 17 & 5 & .10 & .6 & 1.2 & \text { KAO } & 1.3 \times & 147 & 3\end{array}$

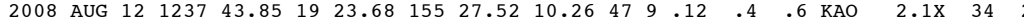

$\begin{array}{lllllllllllllllll}2008 \text { AUG } 12 & 1238 & 58.22 & 19 & 23.71 & 155 & 27.32 & 9.94 & 46 & 9 & .11 & .3 & .6 & \text { KAO } & 1.8 \mathrm{X} & 47 & 2\end{array}$

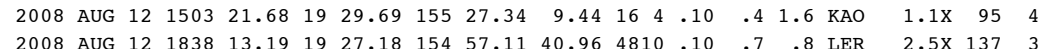

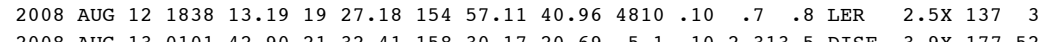

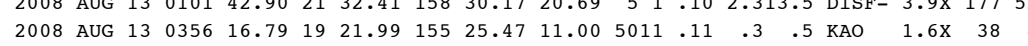

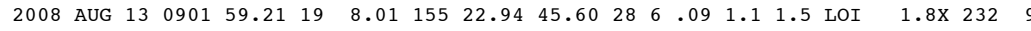

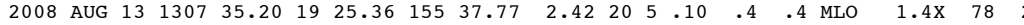

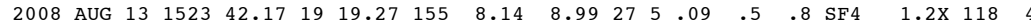

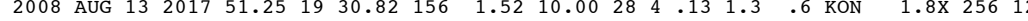

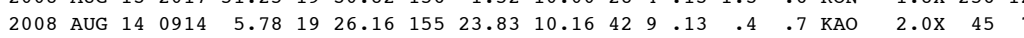

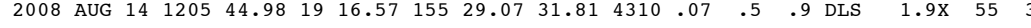

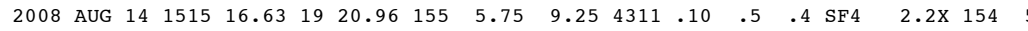

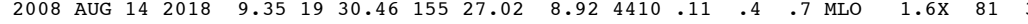

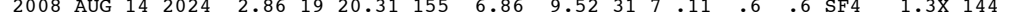

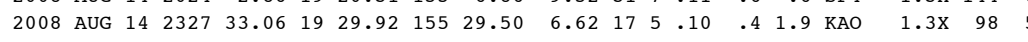

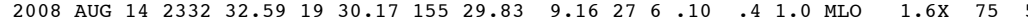

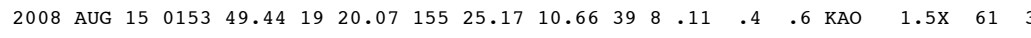

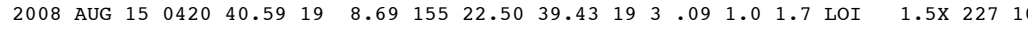

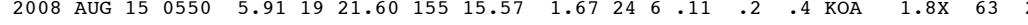

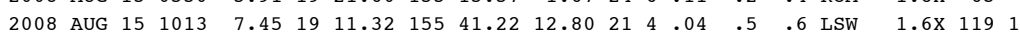


---ORIGIN TIME (HST)-- -LAT N-- --LON W-- DEPTH N N RMS ERH ERZ LOC PREF AZ MIN YEAR MON DA HRMN SEC DEG MIN DEG MIN KM RD S SEC KM KM REMKS MAG GAP DS

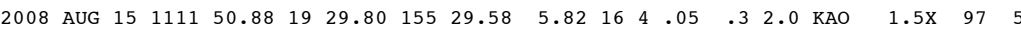

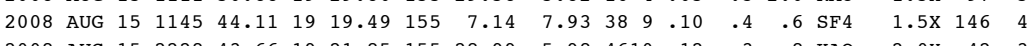

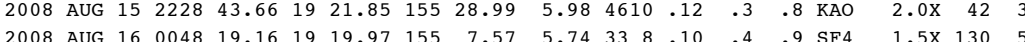

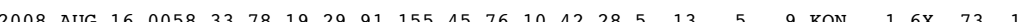

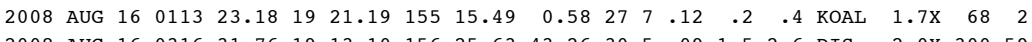

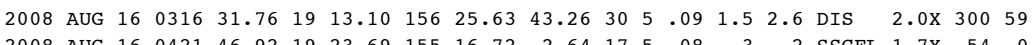

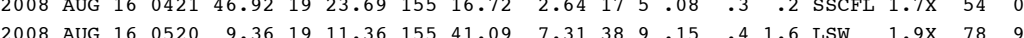
$\begin{array}{lllllllllllll}2008 & \end{array}$

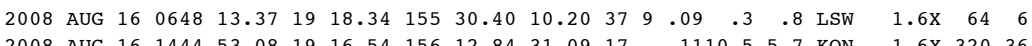

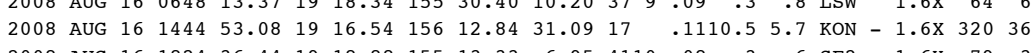

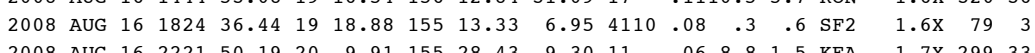

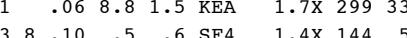

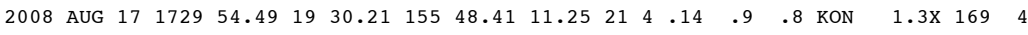

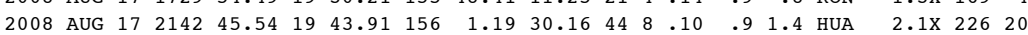

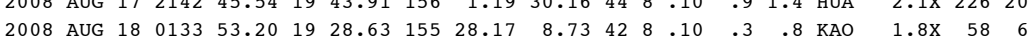
2008 AUG $18 \begin{array}{lllllllllllllllll} & 0633 & 49.80 & 19 & 23.75 & 155 & 16.00 & 1.73 & 19 & 5 & .13 & .3 & .2 & \text { SECB } & 2.4 \times & 101 & 1\end{array}$

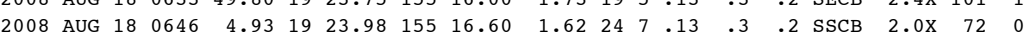

$\begin{array}{llllllllllllllllll}2008 \text { AUG } 18 & 1643 & 31.37 & 19 & 22.79 & 155 & 2.55 & 6.13 & 29 & 7 & .12 & .6 & 1.2 & \text { SF5 } & 1.4 \mathrm{X} & 167 & 5\end{array}$

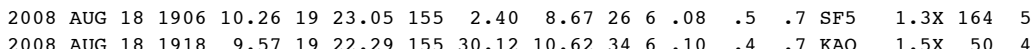

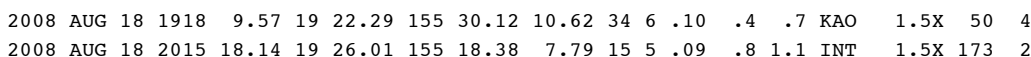
2008 AUG $190006 \quad 42.62 \quad 19 \quad 42.52 \quad 156 \begin{array}{llllllllllll}1.45 & 9.46 & 27 & 5 & .11 & 1.1 & .7 & \text { HUA } & 1.7 \times & 265 & 27\end{array}$

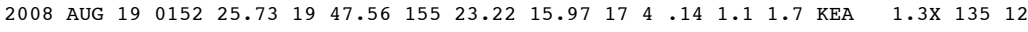

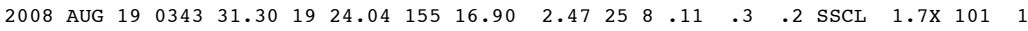

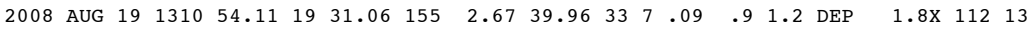

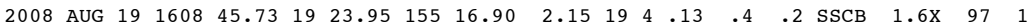
2008 AUG $19 \quad 1811 \quad 28.96 \quad 19 \quad 31.92 \quad 155 \quad 54.17 \quad 18.63 \quad 21 \quad 3 \quad .12 \quad 2.0 \quad 5.4$ KON $1.4 \times \quad 266 \quad 30$

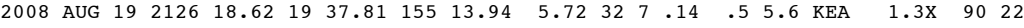

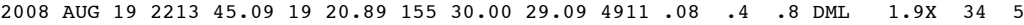

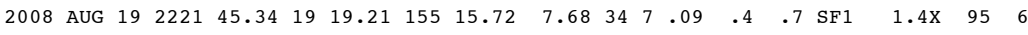

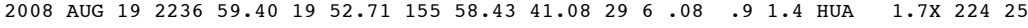

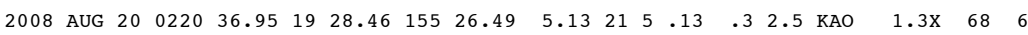

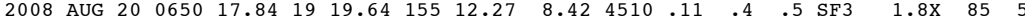
2008 AUG $201144 \quad 42.27 \quad 19 \begin{array}{lllllllllllll}21.06 & 155 & 5.90 & 8.58 & 4211 & .08 & .4 & .5 & \text { SF4 } & 1.8 \mathrm{x} & 149 & 5\end{array}$

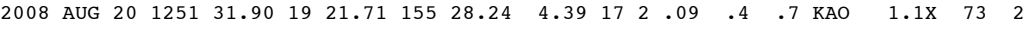

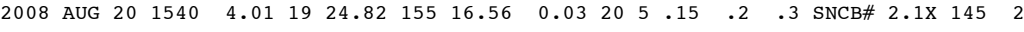

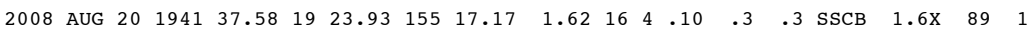

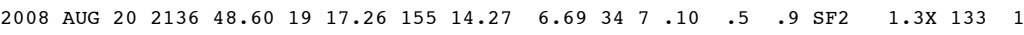

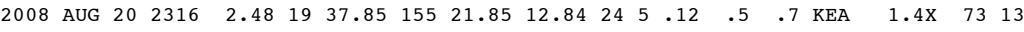

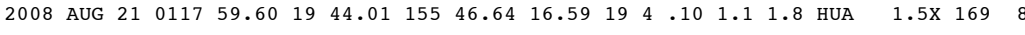

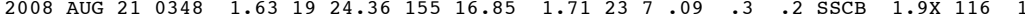

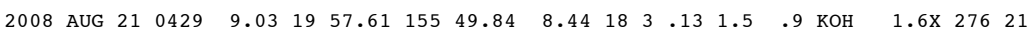

---ORTGIN TIME (HST)-- -LAT N-- --LON W-- DEPTH N N RMS ERH ERZ LOC PREF AZ MIN YEAR MON DA HRMN SEC DEG MIN DEG MIN KM RD S SEC KM KM REMRS MAG GAP DS

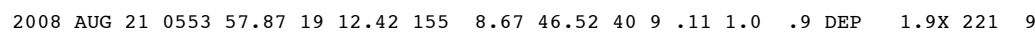

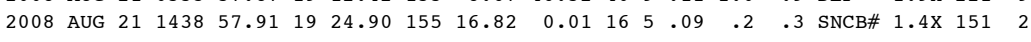

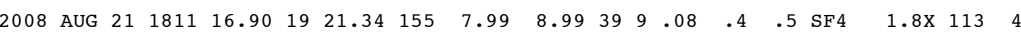
$\begin{array}{llllllllllllllll}2008 \text { AUG } 21 & 1928 & 21.32 & 19 & 20.39 & 155 & 7.83 & 8.11 & 3810 & .09 & .4 & .7 & \text { SF4 } & 1.8 \times & 122\end{array}$

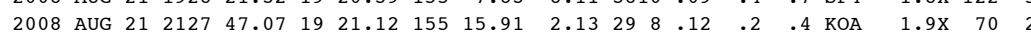

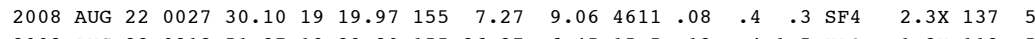

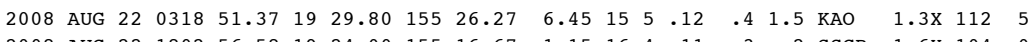
$\begin{array}{lllllllllllllllll}2008 \text { AUG } 22 & 1802 & 56.58 & 19 & 24.00 & 155 & 16.67 & 1.15 & 16 & 4 & .11 & .3 & .2 & \text { SSCB } & 1.6 \times & 104 & 0\end{array}$

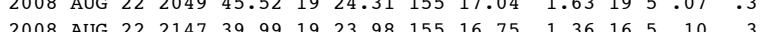

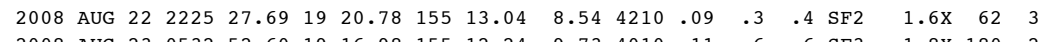

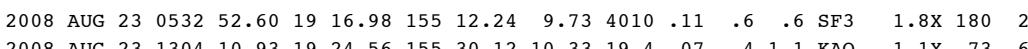

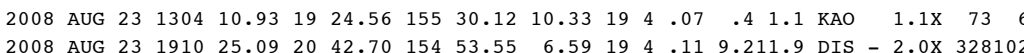

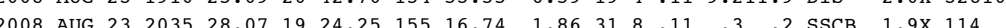

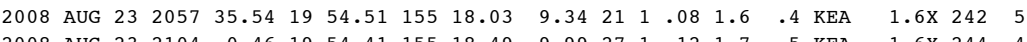

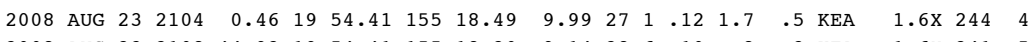

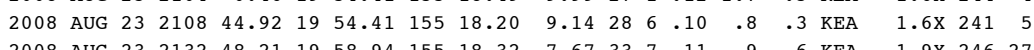

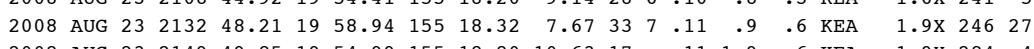

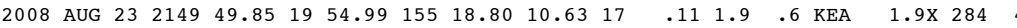

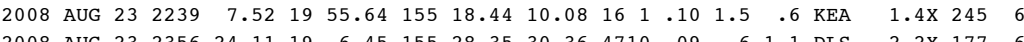

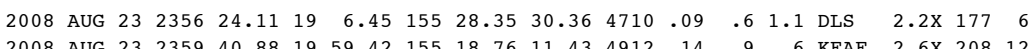

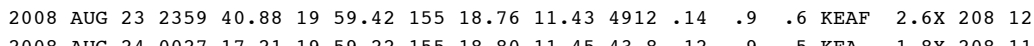

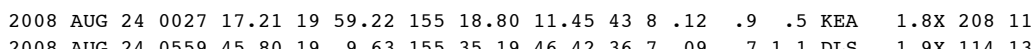

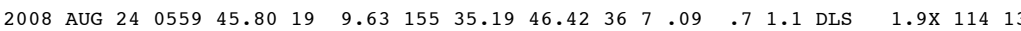

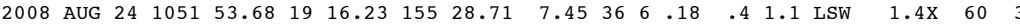

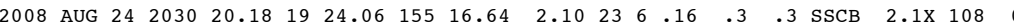

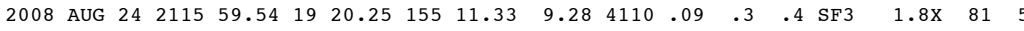

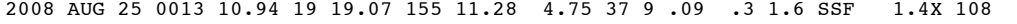

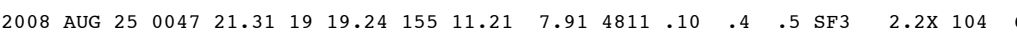

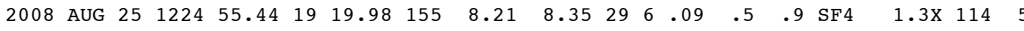

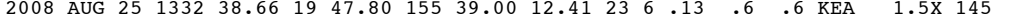

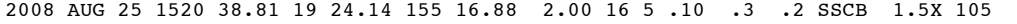

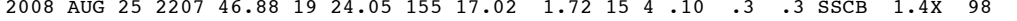

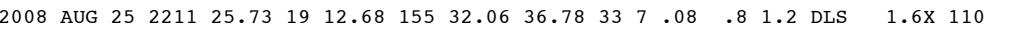

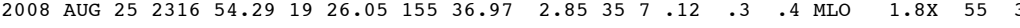

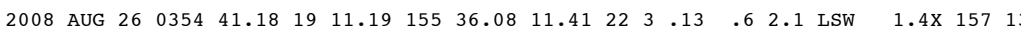

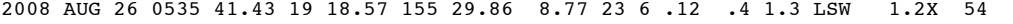

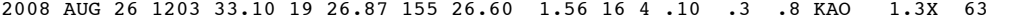

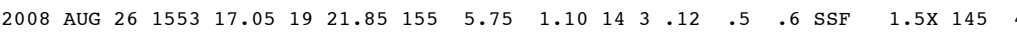

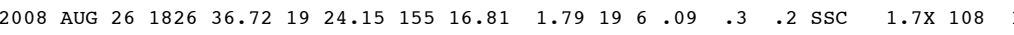

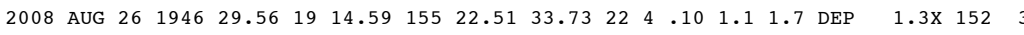

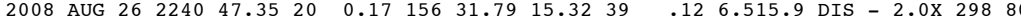

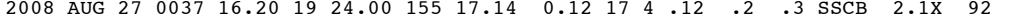

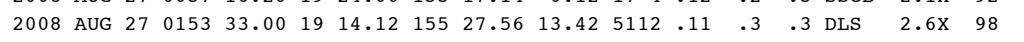


---ORIGIN TIME (HST)-- -LAT N-- --LON W-- DEPTH N N RMS ERH ERZ LOC PREF AZ MIN YEAR MON DA HRMN SEC DEG MIN DEG MIN KM RD S SEC KM KM REMKS MAG GAP DS

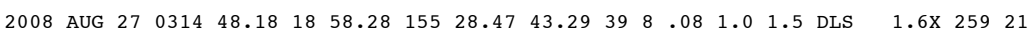

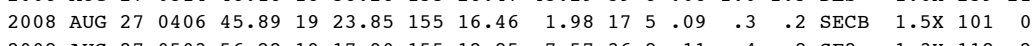

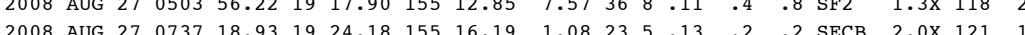

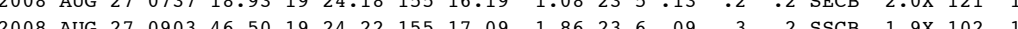

2008 AUG $27 \begin{array}{lllllllllllllllll}1456 & 10.94 & 19 & 22.10 & 155 & 30.32 & 9.49 & 39 & 9 & .11 & .4 & .9 & \text { KAO } & 1.9 \mathrm{X} & 52 & 5\end{array}$ $\begin{array}{lllllllllllllllll}2008 \text { AUG } 27 & 1704 & 32.76 & 19 & 19.22 & 155 & 8.69 & 6.50 & 32 & 8 & .07 & .4 & .8 & \text { SF } 4 & 1.3 \times & 102 & 4 \\ 2008 \text { AUG } 27 & 2338 & 23.51 & 19 & 24.18 & 155 & 16.77 & 1.83 & 17 & 5 & .10 & .3 & .2 & \text { SSCB } & 1.3 \times & 109 & 1\end{array}$

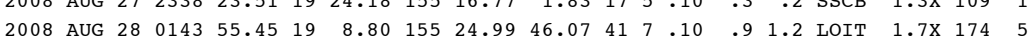

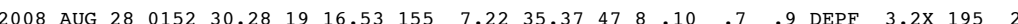

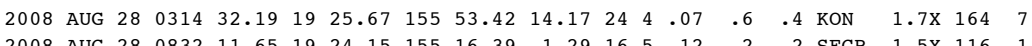

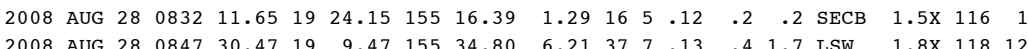

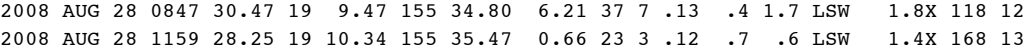

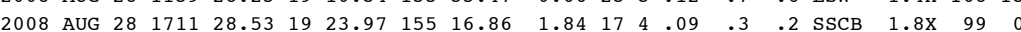

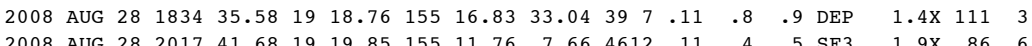

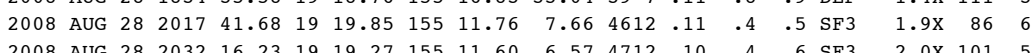

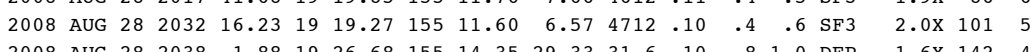

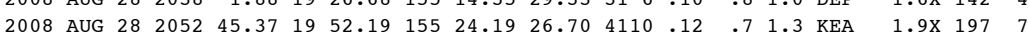

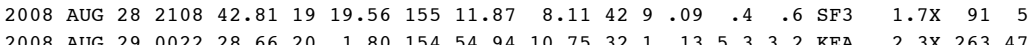

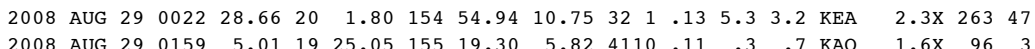

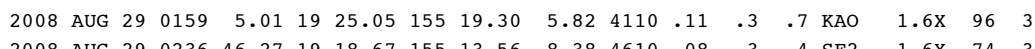

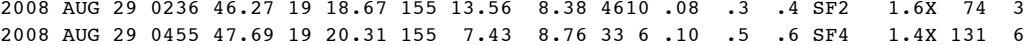

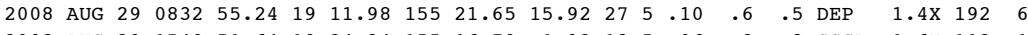

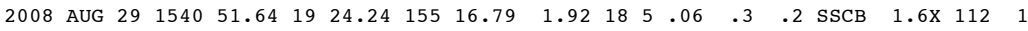

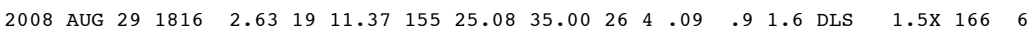

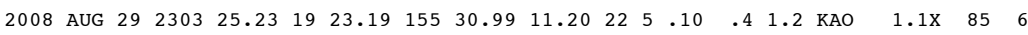

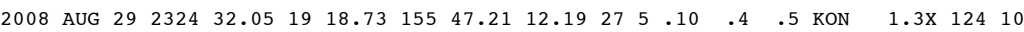

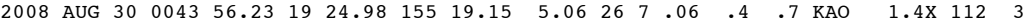

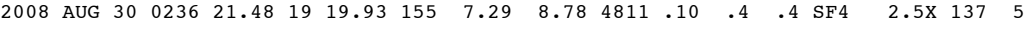

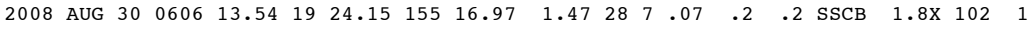

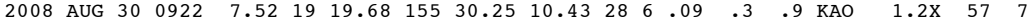

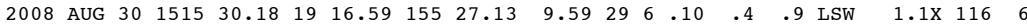

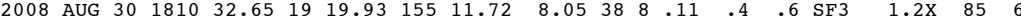

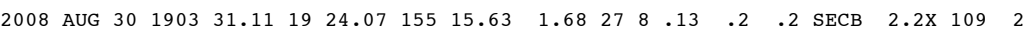
2008 AUG $30 \begin{array}{llllllllllllllll}2038 & 46.50 & 19 & 24.40 & 155 & 16.83 & 1.27 & 15 & 5 & .12 & .3 & .2 & \text { SSCB } & 1.6 \mathrm{X} & 118 & 1\end{array}$

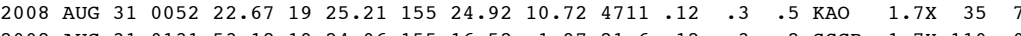

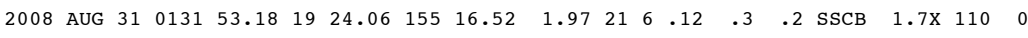

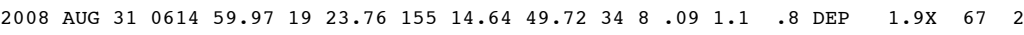

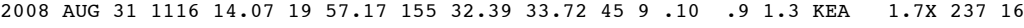

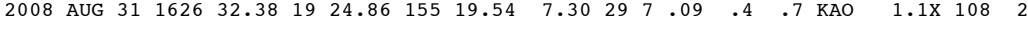

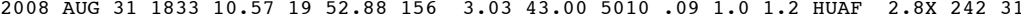

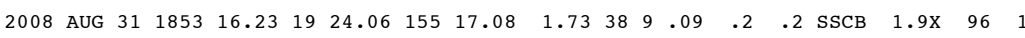

---ORIGIN TIME (HST)-- -LAT N-- --LON W-- DEPTH N N RMS ERH ERZ LOC PREF AZ MIN YEAR MON DA HRMN SEC DEG MIN DEG MIN KM RD S SEC KM KM REMKS MAG GAP DS

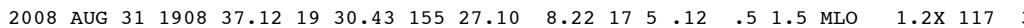

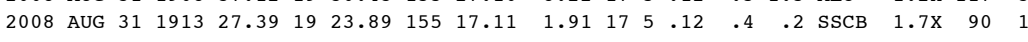
$\begin{array}{llllllllllllllllll}2008 & \text { SEP } & 1 & 0529 & 2.47 & 19 & 16.18 & 155 & 22.53 & 35.20 & 44 & 9 & .12 & .8 & 1.0 & \text { DEP } & 1.8 \mathrm{X} & 133\end{array}$ $\begin{array}{lllllllll} & 2008 \text { SEP } 1 & 0616 & 21.34 & 19 & 24.11 & 155 & 16.84 & 1.80\end{array}$ $\begin{array}{llllllllllllll} & 0654 & 10.27 & 19 & 43.71 & 156 & 5.38 & 7.12 & 4910 & .12 & 1.0\end{array}$ $\begin{array}{lllllllllllllllllll}2008 & \text { SEP } & 1 & 0657 & 24.47 & 19 & 53.16 & 156 & 11.25 & 0.68 & 20 & .15 & 3.9 & 6.9 & \text { HUA \# } & 1.9 \mathrm{X} & 295 & 43\end{array}$ $\begin{array}{lllllllllllllllll}2008 & \text { SEP } & 1 & 0716 & 6.27 & 39.64 & 156 & 2.97 & 9.90 & 22 & .11 & 3.3 & 1.0 & \text { HUA } & 1.8 \times & 265 & 24\end{array}$

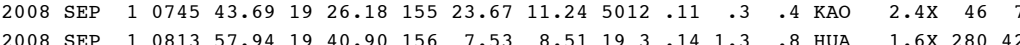
$\begin{array}{llllllllll} & \end{array}$

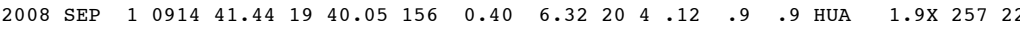

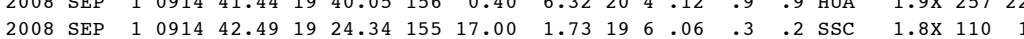

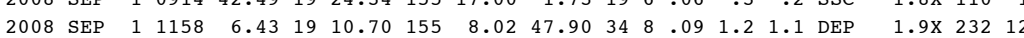
$\begin{array}{llllllllllllllllll}2008 & \text { SEP } & 1 & 1235 & 47.36 & 19 & 39.56 & 156 & 2.90 & 8.46 & 30 & .12 & 2.4 & .6 & \text { HUA } & 2.1 \times & 265 & 23\end{array}$

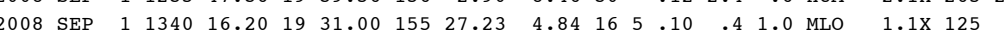

$\begin{array}{llllllllllllllllllll}2008 & \text { SEP } & 1 & 1345 & 3.87 & 19 & 31.07 & 155 & 28.07 & 5.26 & 15 & 5 & .10 & .4 & 1.1 & \text { MLO } & 1.3 \mathrm{X} & 93 & 2\end{array}$

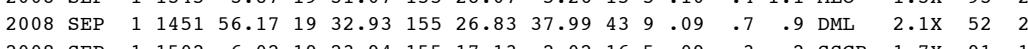
$\begin{array}{lllllllllllllllllll}2008 & \text { SEP } & 1 & 1502 & 6.02 & 19 & 23.94 & 155 & 17.13 & 2.02 & 16 & 5 & .09 & .3 & .2 & \text { SSCB } & 1.7 \mathrm{x} & 91 & 1\end{array}$

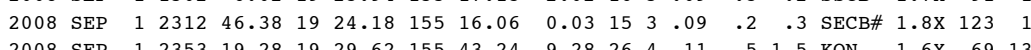
$\begin{array}{lllllllllllllllllll}2008 & \text { SEP } & 1 & 2353 & 19.28 & 19 & 29.62 & 155 & 43.24 & 9.28 & 26 & 4 & .11 & .5 & 1.5 & \text { KON } & 1.6 \mathrm{X} & 69 & 13\end{array}$

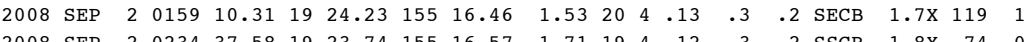

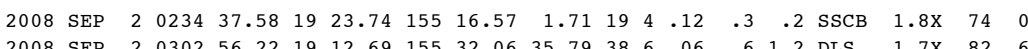

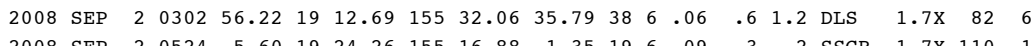
$\begin{array}{llllllllllllllllll}2008 & \text { SEP } & 2 & 0524 & 5.60 & 19 & 24.26 & 155 & 16.88 & 1.35 & 19 & 6 & .09 & .3 & .2 & \text { SSCB } & 1.7 \times & 110 \\ 2008 & \text { SEP } & 2 & 0535 & 34.86 & 19 & 24.15 & 155 & 16.41 & 1.62 & 22 & 7 & 12 & .3 & 2 & \text { SECB } & 2.2 \mathrm{X} & 117\end{array}$

$\begin{array}{lllllllllllllllllll}2008 & \text { SEP } & 2 & 0545 & 18.92 & 19 & 23.81 & 155 & 17.02 & 1.73 & 19 & 5 & .09 & .3 & .2 & \text { SSCB } & 1.4 \mathrm{X} & 89 & 1\end{array}$ $\begin{array}{lllllllllllllllll}2008 & \text { SEP } & 2 & 0952 & 11.64 & 19 & 12.88 & 155 & 29.29 & 37.46 & 4611 & .08 & .6 & 1.1 & \text { DLS } & 1.9 \mathrm{X} & 81\end{array}$ $\begin{array}{llllllllllllllllll}2008 & \text { SEP } & 2 & 1715 & 39.26 & 19 & 23.82 & 155 & 16.74 & 1.18 & 17 & 6 & .14 & .3 & .2 & \text { SSCB } & 1.6 \mathrm{X} & 86\end{array}$

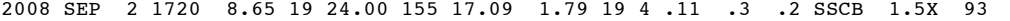

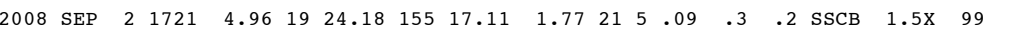

$\begin{array}{llllllllllllllllll}2008 & \text { SEP } & 2 & 1727 & 27.54 & 19 & 24.11 & 155 & 16.98 & 1.56 & 23 & 5 & .12 & .3 & .2 & \text { SSCB } & 1.9 \times & 101\end{array}$

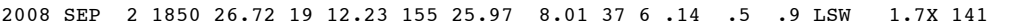

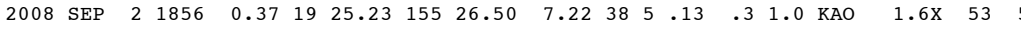

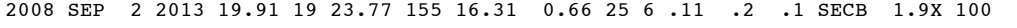

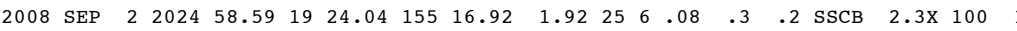

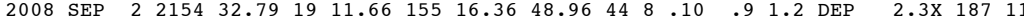

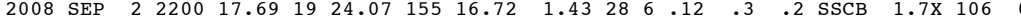

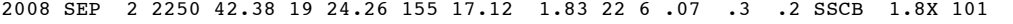

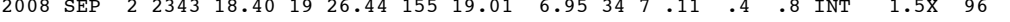

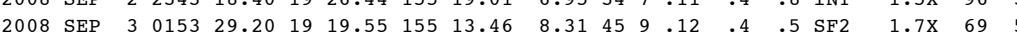

$\begin{array}{llllllllllllllllll}2008 & \text { SEP } & 3 & 0211 & 37.84 & 19 & 15.98 & 155 & 7.03 & 42.51 & 43 & 9 & .10 & .8 & .9 & \text { DEP } & 1.7 \times & 208\end{array}$

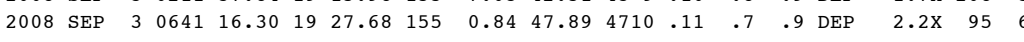

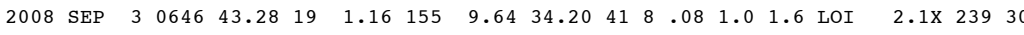

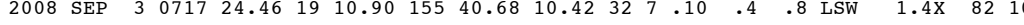

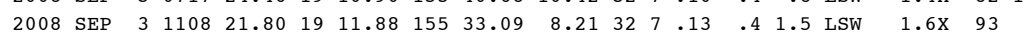


---ORIGIN TIME (HST)-- -LAT N-- --LON W-- DEPTH N N RMS ERH ERZ LOC PREF AZ MIN YEAR MON DA HRMN SEC DEG MIN DEG MIN KM RD S SEC KM KM REMKS MAG GAP DS

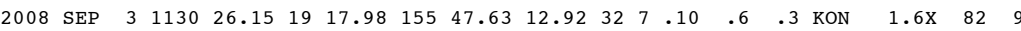

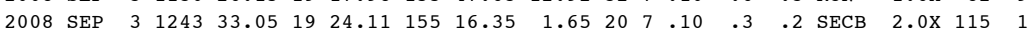

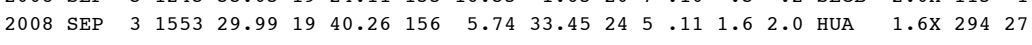

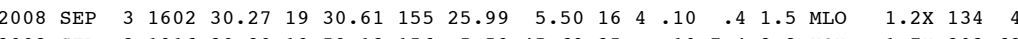
$\begin{array}{llllllllllllll}2008 & \text { SEP } & 3 & 1916 & 30.30 & 19 & 59.13 & 156 & 5.59 & 45.69 & 25 & .10 & 7.4 & 3.8 \\ \text { кон } & 1.7 \times & 302 & 63\end{array}$

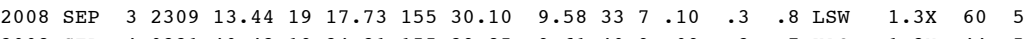

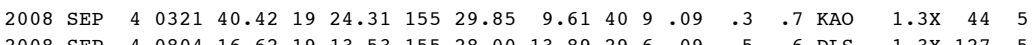

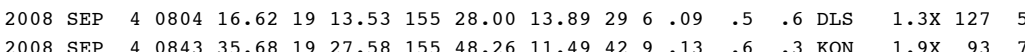

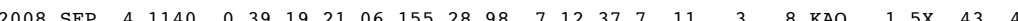

2008 SEP $4 \begin{array}{lllllllllllllllll}4 & 1441 & 59.31 & 19 & 31.61 & 155 & 41.12 & 8.67 & 23 & 4 & .13 & .7 & 1.3 & \text { MLO } & 1.4 \times & 166 & 9\end{array}$ $\begin{array}{llllllllllllllllll}2008 & \text { SEP } & 4 & 1738 & 55.00 & 19 & 15.94 & 156 & 14.86 & 32.16 & 28 & .12 & 8.4 & 3.6 & \text { KON } & 1.8 \times & 310 & 40\end{array}$

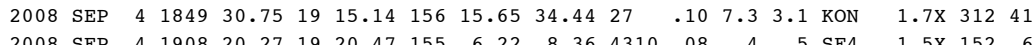

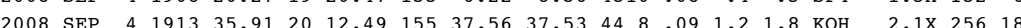

$\begin{array}{lllllllllllllllllll}2008 & \text { SEP } & 4 & 2027 & 59.86 & 19 & 32.43 & 155 & 28.14 & 5.64 & 17 & 4 & .10 & .5 & 1.0 & \text { MLO } & 1.2 \times & 132 & 1\end{array}$

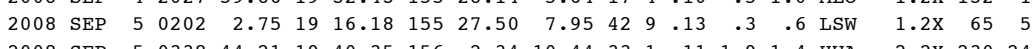

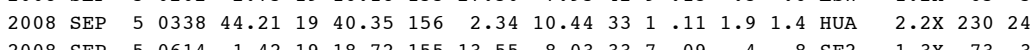

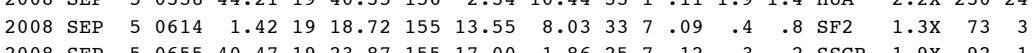

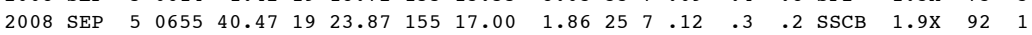

$\begin{array}{llllllllllllllllll}2008 & \text { SEP } & 5 & 0658 & 14.61 & 19 & 19.13 & 155 & 8.46 & 8.03 & 4111 & .07 & .4 & .5 & \text { SF } 4 & 1.6 \times & 108 & 3\end{array}$

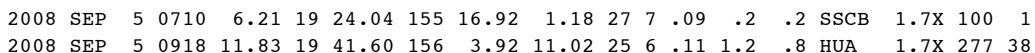
$\begin{array}{lllllllllllllllllll}2008 & \text { SEP } & 5 & 0918 & 11.83 & 19 & 41.60 & 156 & 3.92 & 11.02 & 25 & 6 & .11 & 1.2 & .8 & \text { HUA } & 1.7 x & 277 & 38 \\ 2008 & \text { SEP } & 5 & 2238 & 7.60 & 19 & 17.19 & 155 & 27.47 & 6.68 & 32 & 6 & .11 & .3 & 1.1 & \text { LSW } & 1.4 x & 93 & 6\end{array}$

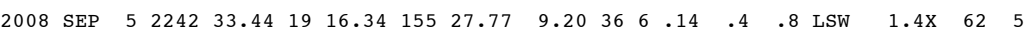

2008 SEP $6 \begin{array}{lllllllllllllllll} & 0357 & 39.03 & 19 & 19.46 & 155 & 11.72 & 9.90 & 41 & 9 & .09 & .4 & .4 & \text { SF3 } & 1.6 \mathrm{X} & 95 & 5\end{array}$

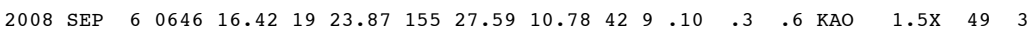

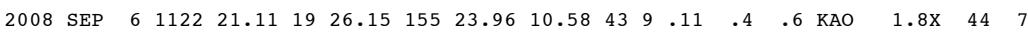

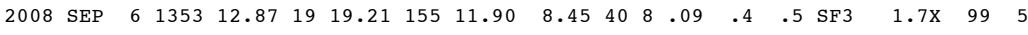
$\begin{array}{lllllllllllllllllll}2008 & \text { SEP } & 6 & 1441 & 1.36 & 19 & 19.83 & 155 & 12.50 & 8.77 & 42 & 8 & .09 & .4 & .5 & \text { SF2 } & 1.7 \mathrm{X} & 80 & 5\end{array}$

$\begin{array}{lllllllllllllllllll}2008 & \text { SEP } & 6 & 1450 & 7.08 & 19 & 26.08 & 155 & 28.08 & 10.54 & 31 & 6 & .10 & .4 & .8 & \text { KAO } & 1.3 \mathrm{X} & 61 & 7\end{array}$ 2008 SEP $6 \begin{array}{lllllllllllllllll}1845 & 23.65 & 19 & 20.23 & 155 & 7.84 & 8.09 & 32 & 7 & .09 & .5 & .7 & \text { SF } 4 & 1.6 \mathrm{X} & 123 & 5\end{array}$

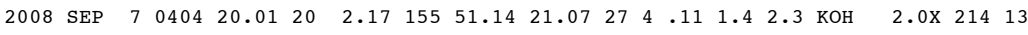
2008 SEP $7 \quad \begin{array}{llllllllllllllllll}0420 & 11.70 & 18 & 56.21 & 155 & 13.61 & 41.19 & 25 & 5 & .09 & 1.5 & 2.3 & \text { LOI } & 1.7 \mathrm{X} & 293 & 35\end{array}$

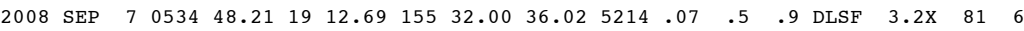

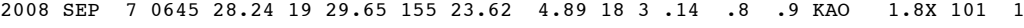

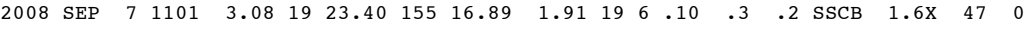

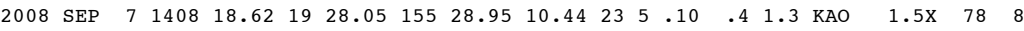

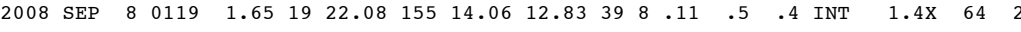

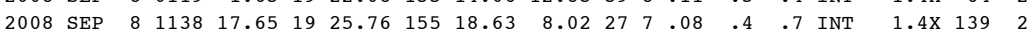

$\begin{array}{lllllllllllllllllll}2008 & \text { SEP } & 8 & 1202 & 20.38 & 19 & 26.76 & 155 & 30.10 & 14.68 & 16 & 4 & .09 & .6 & 1.3 & \text { DML } & .9 \mathrm{x} & 71 & 6\end{array}$

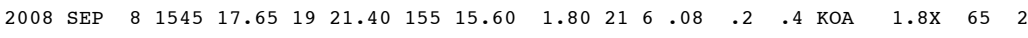

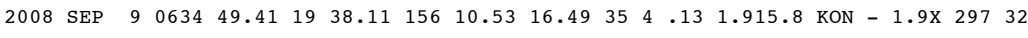

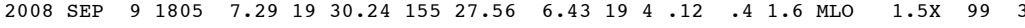

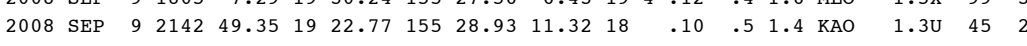

---ORIGIN TIME (HST)-- -LAT N-- --LON W-- DEPTH N N RMS ERH ERZ LOC PREF AZ MIN YEAR MON DA HRMN SEC DEG MIN DEG MIN KM RD S SEC KM KM REMKS MAG GAP DS

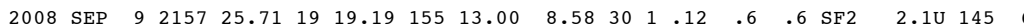

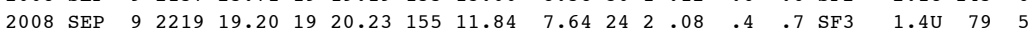

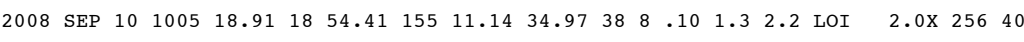

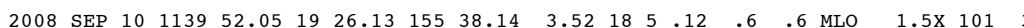

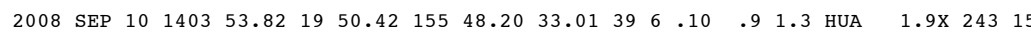

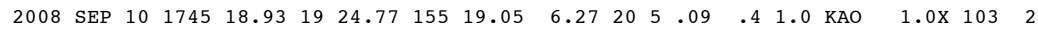

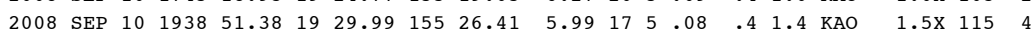

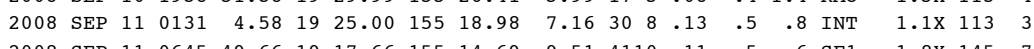

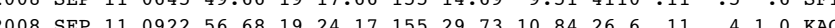

$\begin{array}{lllllllllllllllll}2008 & \text { SEP } 11 & 1116 & 39.75 & 19 & 20.01 & 155 & 7.85 & 9.78 & 5213 & .11 & .4 & .4 & \text { SF4 } & 2.8 \times & 123 & 5\end{array}$

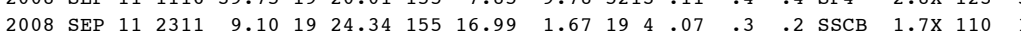

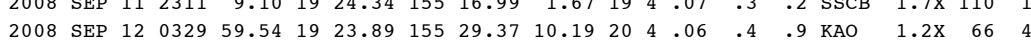

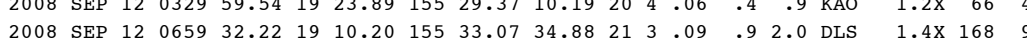

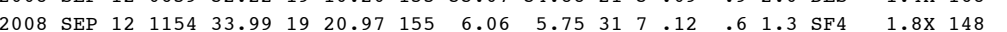

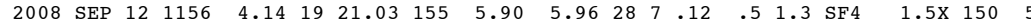

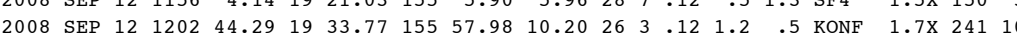

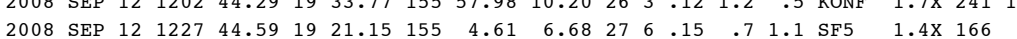

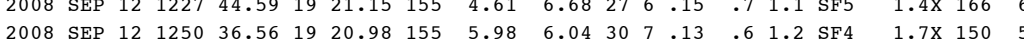

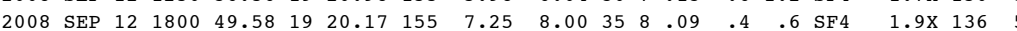

$\begin{array}{llllllllllllllllll}2008 & \text { SEP } 12 & 1838 & 42.95 & 19 & 31.61 & 155 & 47.31 & 10.74 & 24 & 4 & .14 & .5 & .6 & \text { KON } & 1.1 \mathrm{x} & 91 & 3\end{array}$

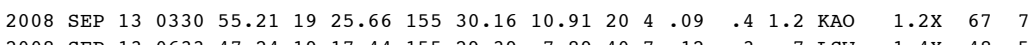

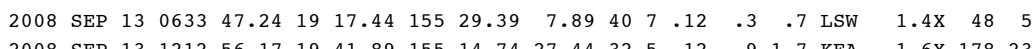

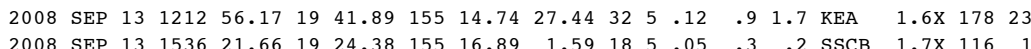

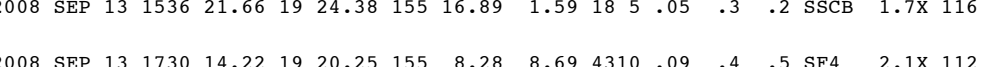

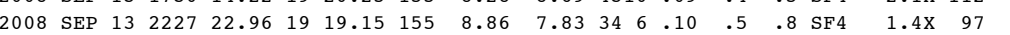

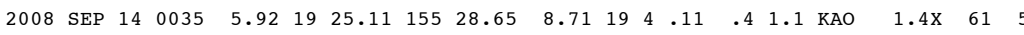

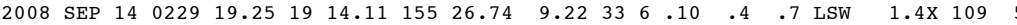

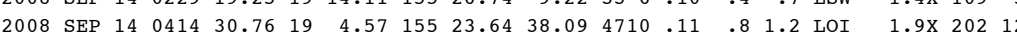

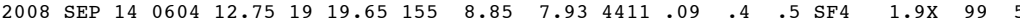

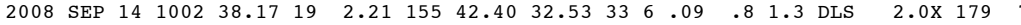

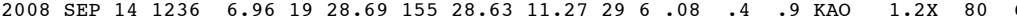

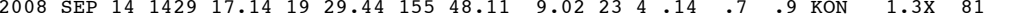

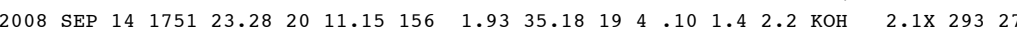

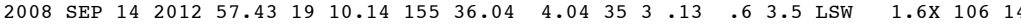

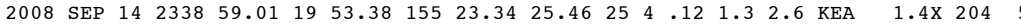

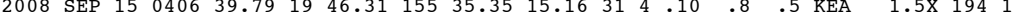

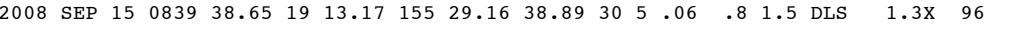

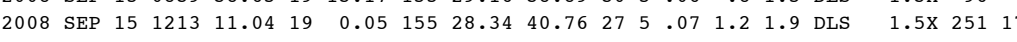

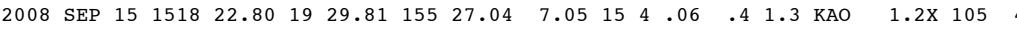

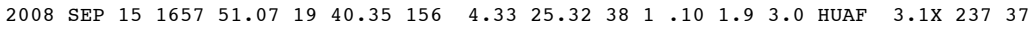

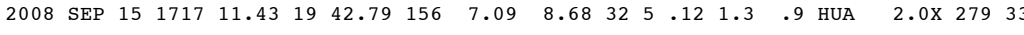
2008 SEP $15 \quad \begin{array}{llllllllllllllll}1734 & 21.23 & 19 & 35.81 & 155 & 58.70 & 24.78 & 24 & .11 & 4.3 & 3.8 & \text { KON } & 2.1 \times & 286 & 38\end{array}$

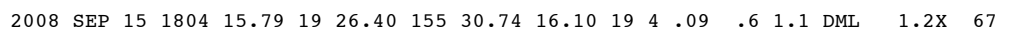


---ORIGIN TIME (HST)-- -LAT N-- --LON W-- DEPTH N N RMS ERH ERZ LOC PREF AZ MIN YEAR MON DA HRMN SEC DEG MIN DEG MIN KM RD S SEC KM KM REMKS MAG GAP DS

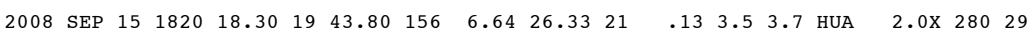

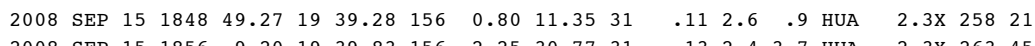
$\begin{array}{lllllllllllll}2008 & & \end{array}$

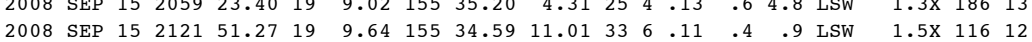

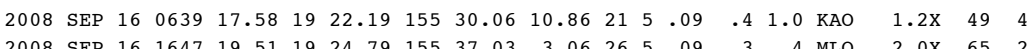

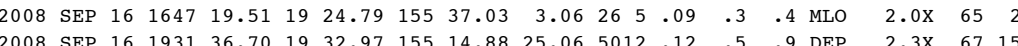

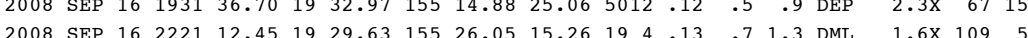

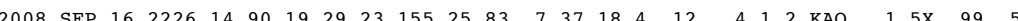

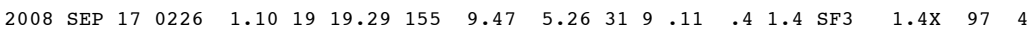

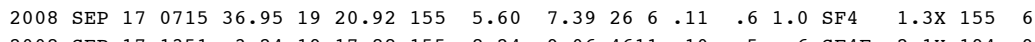

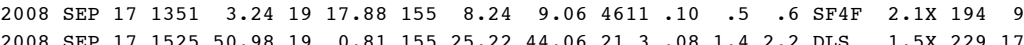
2008 SEP $18 \quad \begin{array}{lllllllllllllllllll} & 0431 & 23.28 & 19 & 29.69 & 155 & 26.38 & 8.59 & 24 & 7 & 11 & 4 & 1.0 & \text { KAO } & 1.3 \times & 90 & 5\end{array}$

$\begin{array}{llllllllllllllllll}2008 & \text { SEP } 18 & 0549 & 56.14 & 19 & 25.97 & 155 & 24.45 & 11.10 & 36 & 7 & .12 & .4 & .8 & \text { KAO } & 1.5 \mathrm{X} & 76 & 7\end{array}$

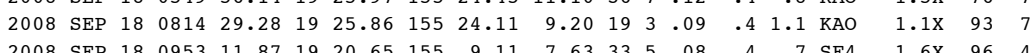

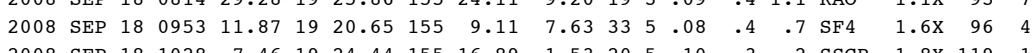

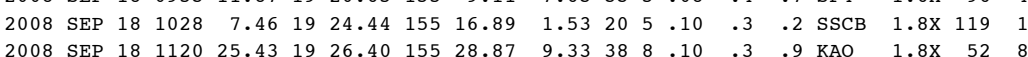

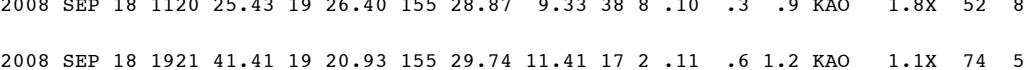

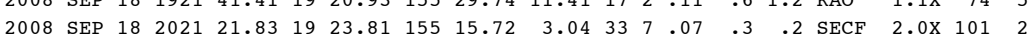

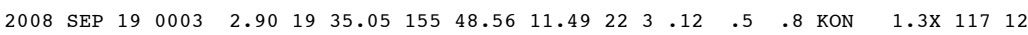

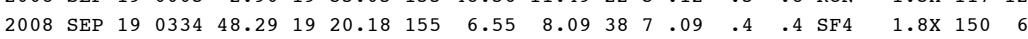

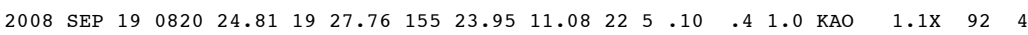

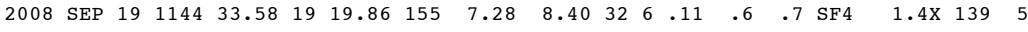

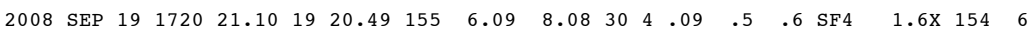
$\begin{array}{llllllllllllllllll}2008 & \text { SEP } 19 & 1723 & 23.65 & 19 & 25.00 & 155 & 29.66 & 11.80 & 24 & 3 & .10 & .5 & 1.0 & \text { KAO } & 1.2 \mathrm{X} & 69 & 6\end{array}$ 2008 SEP $191759 \begin{array}{llllllllllllll}17.19 & 19 & 19.49 & 155 & 6.52 & 9.78 & 4811 & .10 & .6 & .3 & \text { SF4F } & 4.3 \mathrm{U} & 160 & 5\end{array}$

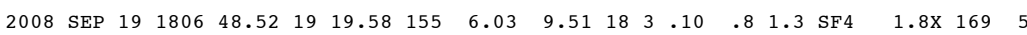

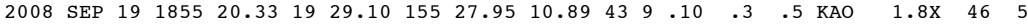

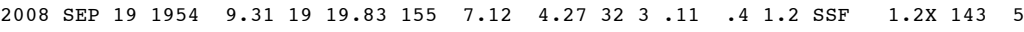

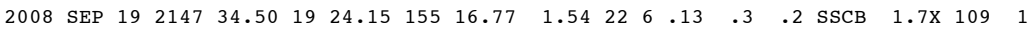

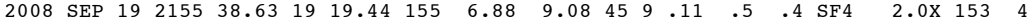

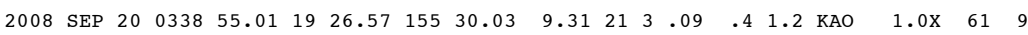

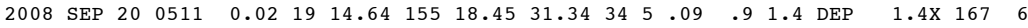
2008 SEP $201807 \quad 56.001946 .14 \quad 15614.64 \quad 24.84 \quad 15 \quad .1611 .8 \quad 6.0$ HUA $-1.7 \times 31743$

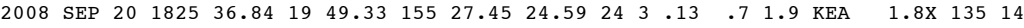

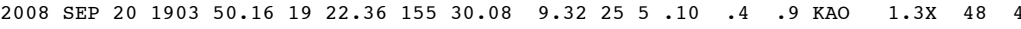

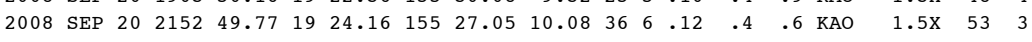

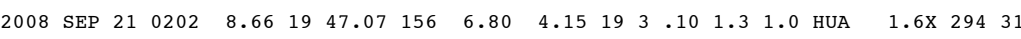

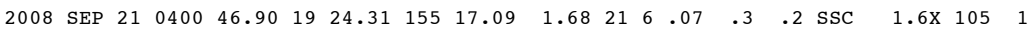

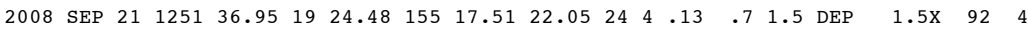

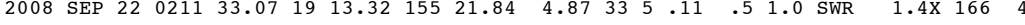

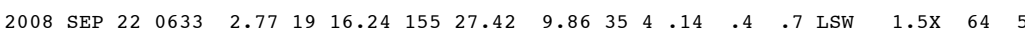

--ORIGIN TIME (HST)-- -LAT N-- --LON W-- DEPTH N N RMS ERH ERZ LOC PREF AZ MIN YEAR MON DA HRMN SEC DEG MIN DEG MIN KM RD S SEC KM KM REMKS MAG GAP DS

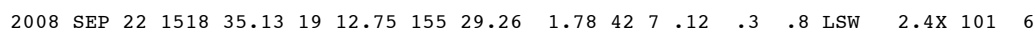

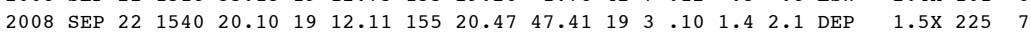

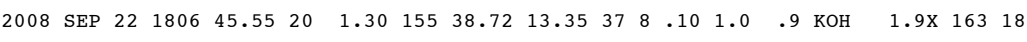
$\begin{array}{llllllllllll} & 008 \text { SEP } 22 & 2040 & 16.38 & 19 & 19.29 & 155 & 13.15 & 8.67 & 40 & 7 & .10\end{array}$

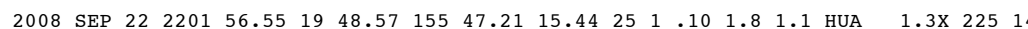

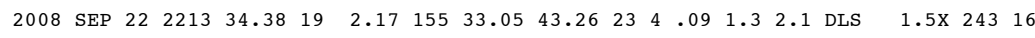
$\begin{array}{lllllllllllllll}2008 & \text { SEP } & 23 & 0102 & 44.14 & 19 & 22.53 & 155 & 29.69 & 9.81 & 45 & 8 & .11 & .3 & .6 \\ \text { KAO } & 2.0 \mathrm{X} & 33 & 4\end{array}$ $\begin{array}{llllllllllllllllll}2008 & \text { SEP } 23 & 0103 & 7.35 & 19 & 22.67 & 155 & 29.29 & 11.74 & 31 & 5 & .13 & .4 & .8 & \text { KAO } & 1.4 \mathrm{X} & 68 & 3\end{array}$ $\begin{array}{lllllllllll} & \end{array}$

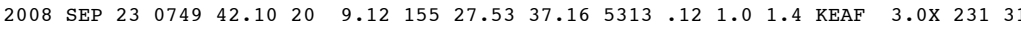

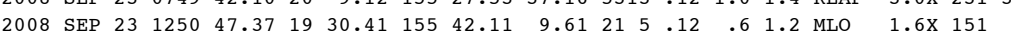

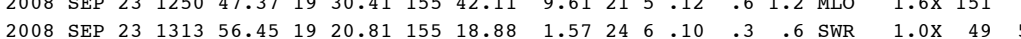

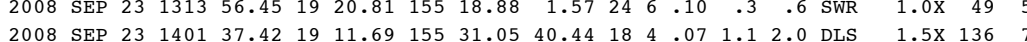

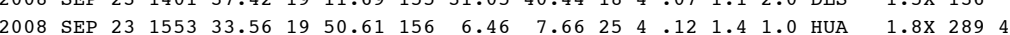

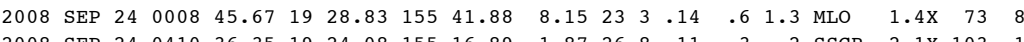

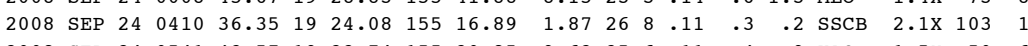

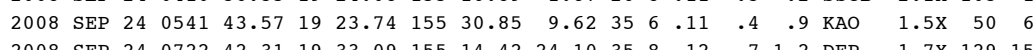

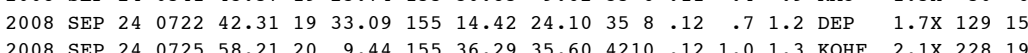

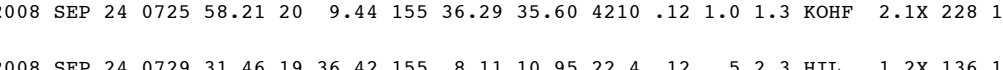

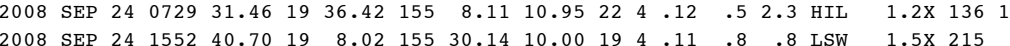

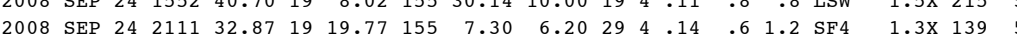

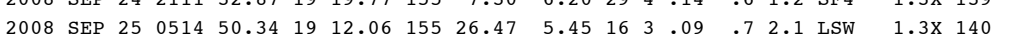

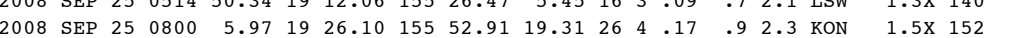

$\begin{array}{lllllllllllllllll}2008 & \text { SEP } 25 & 0839 & 25.56 & 19 & 28.12 & 155 & 26.78 & 9.13 & 17 & 5 & .12 & .5 & 1.5 & \text { KAO } & 1.2 \mathrm{x} & 75\end{array}$

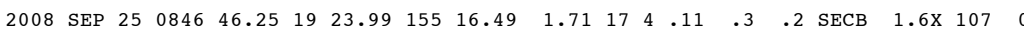

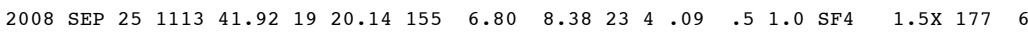

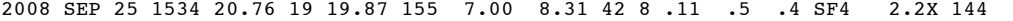

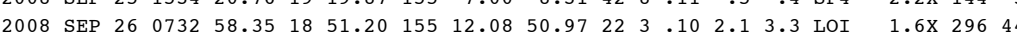

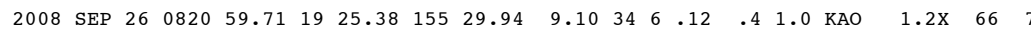

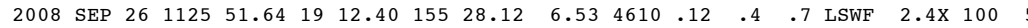

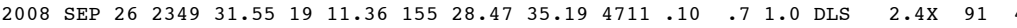

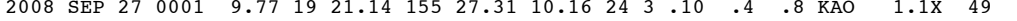

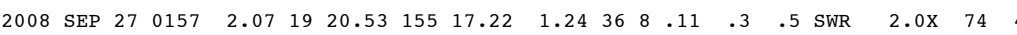

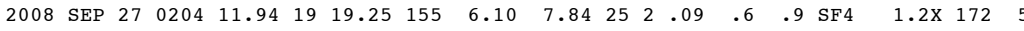

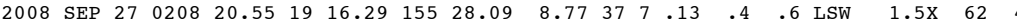

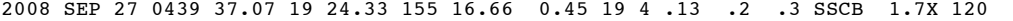

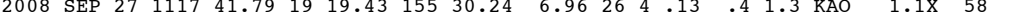

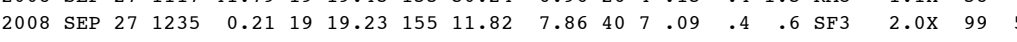

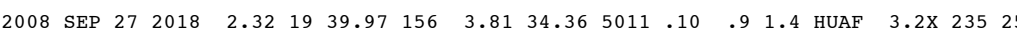

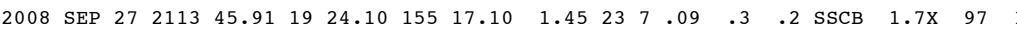

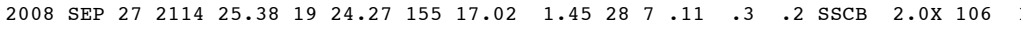

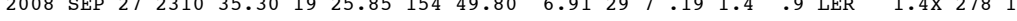

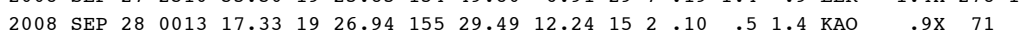


---ORIGIN TIME (HST)-- -LAT N-- --LON W-- DEPTH N N RMS ERH ERZ LOC PREF AZ MIN YEAR MON DA HRMN SEC DEG MIN DEG MIN KM RD S SEC KM KM REMKS MAG GAP DS $\begin{array}{lllllllllllllllllll}2008 & \text { SEP } 28 & 0023 & 56.27 & 19 & 23.97 & 155 & 16.91 & 2.54 & 15 & 5 & .09 & .3 & .2 & \text { SSCB } & 2.1 \times & 97 & 1\end{array}$

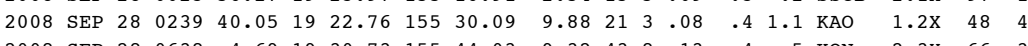

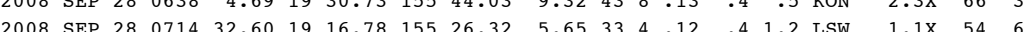

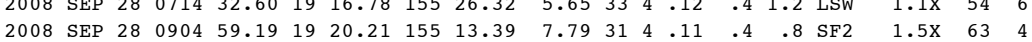

$\begin{array}{llllllllllllllllll}2008 & \text { SEP } 28 & 1130 & 30.94 & 19 & 20.44 & 155 & 6.92 & 8.06 & 32 & 5 & .11 & .5 & .7 & \text { SF4 } & 1.5 \times & 140 & 5\end{array}$

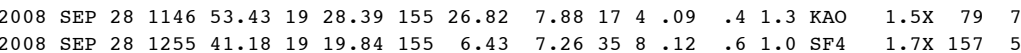

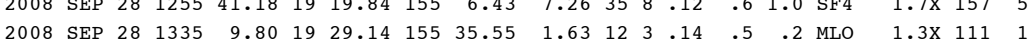

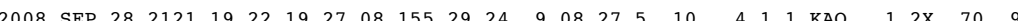

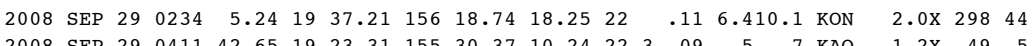

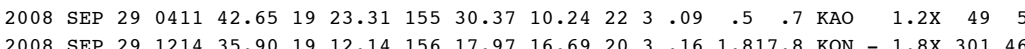

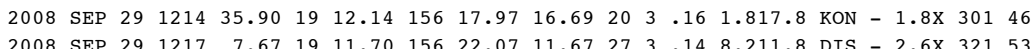

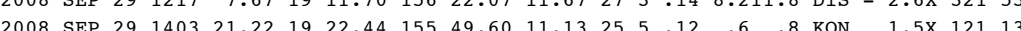

$\begin{array}{llllllllllllllllll}2008 & \text { SEP } 29 & 1441 & 31.98 & 19 & 11.62 & 155 & 31.86 & 0.69 & 32 & 5 & .15 & .4 & .5 & \text { LSW } & 1.6 \mathrm{X} & 94 & 7\end{array}$

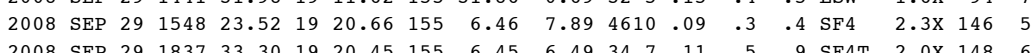

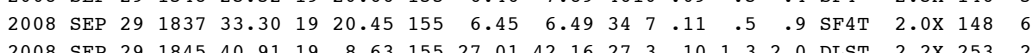

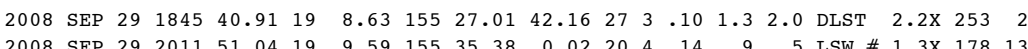
2008 SEP $29 \begin{array}{llllllllllllllll}2011 & 51.04 & 19 & 9.59 & 155 & 35.38 & 0.02 & 20 & 4 & .14 & .9 & .5 & \text { LSW \# } 1.3 \times & 178 & 13\end{array}$

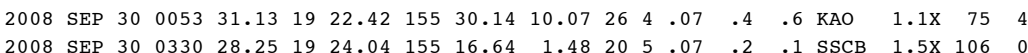

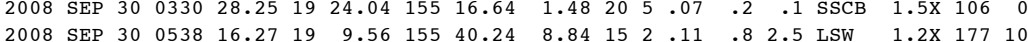

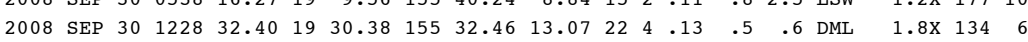

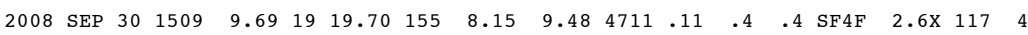

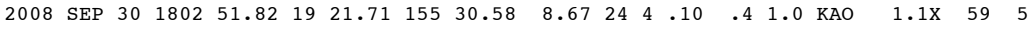

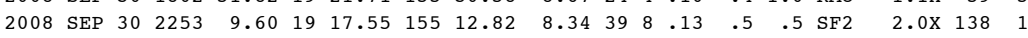

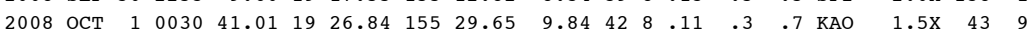
$\begin{array}{lllllllllllllllllll}2008 & \text { ОСТ } & 1 & 0213 & 48.54 & 19 & 17.93 & 155 & 12.67 & 9.71 & 41 & 7 & .10 & .5 & .4 & \text { SF2 } & 2.1 \times & 143 & 8\end{array}$ $\begin{array}{lllllllllllllllllll}2008 & \text { ост } & 1 & 1657 & 34.79 & 19 & 14.04 & 155 & 21.69 & 8.83 & 38 & 5 & .12 & .4 & .8 & \text { SWR } & 1.9 \times & 155 & 4\end{array}$

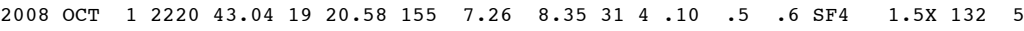
$\begin{array}{lllllllllllllllllll}2008 & \text { OCT } & 2 & 0034 & 34.78 & 19 & 11.57 & 155 & 41.76 & 11.08 & 37 & 7 & .14 & .5 & .6 & \text { LSW } & 2.1 \mathrm{x} & 75 & 8\end{array}$ $\begin{array}{lllllllllllllllllll}2008 & \text { ОСТ } & 2 & 1214 & 45.78 & 19 & 22.31 & 155 & 29.81 & 9.02 & 38 & 6 & .10 & .3 & .8 & \text { KАО } & 1.4 \mathrm{X} & 47 & 4\end{array}$ $\begin{array}{lllllllllllllllllll}2008 & \text { OCT } & 2 & 1258 & 45.97 & 19 & 25.78 & 155 & 28.39 & 9.86 & 18 & 4 & .09 & .5 & 1.3 & \text { KAO } & 1.3 \mathrm{X} & 61 & 6\end{array}$

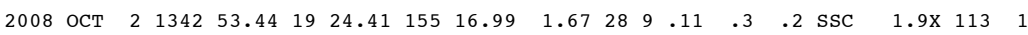

$\begin{array}{lllllllllllllllllll}2008 & \text { OCT } & 2 & 1544 & 1.64 & 19 & 57.81 & 154 & 50.36 & 10.21 & 25 & 3 & .13 & 1.5 & 1.0 & \text { KEA } & 2.1 \times & 287 & 35\end{array}$ $\begin{array}{lllllllllllllllllll}2008 & \text { OCT } & 2 & 1612 & 45.31 & 19 & 25.20 & 155 & 37.54 & 2.77 & 33 & 5 & .13 & .3 & .4 & \text { MLO } & 2.0 x & 74 & 2\end{array}$

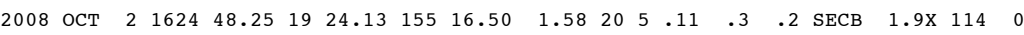

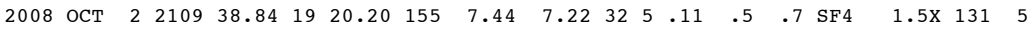

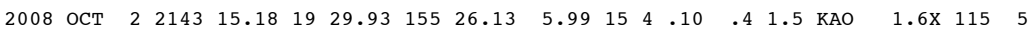

$\begin{array}{lllllllllllllllllll}2008 & \text { OCT } & 2 & 2221 & 30.10 & 19 & 13.40 & 155 & 31.35 & 32.24 & 38 & 6 & .08 & .6 & 1.1 & \text { DLS } & 1.7 \mathrm{X} & 72 & 4\end{array}$ $\begin{array}{lllllllllllllllllll}2008 & \text { OCT } & 2 & 2310 & 51.01 & 19 & 24.39 & 155 & 16.83 & 1.74 & 23 & 7 & .11 & .3 & .2 & \text { SSCB } & 1.8 \mathrm{X} & 117 & 1\end{array}$

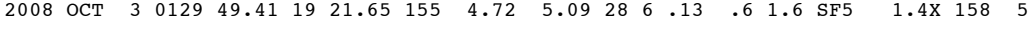

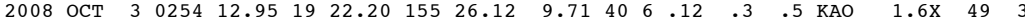

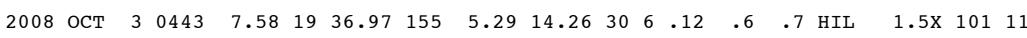

---ORIGIN TIME (HST)-- -LAT N-- --LON W-- DEPTH N N RMS ERH ERZ LOC PREF AZ MIN YEAR MON DA HRMN SEC DEG MIN DEG MIN KM RD S SEC KM KM REMKS MAG GAP DS

$\begin{array}{llllllllllllllllll}2008 & \text { ОСТ } & 3 & 0820 & 41.63 & 19 & 19.59 & 155 & 12.78 & 7.51 & 4110 & 10 & 4 & .6 & \text { SF } 2 & 1.8 \times & 79 & 5\end{array}$

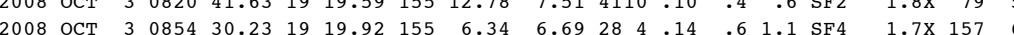

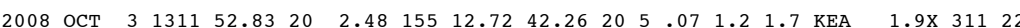

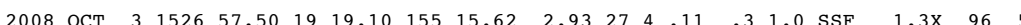

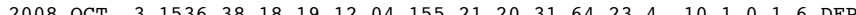

$\begin{array}{lllllllllllllllllll}2008 & \text { ост } & 3 & 1542 & 37.01 & 19 & 19.32 & 155 & 9.67 & 8.05 & 41 & 8 & .09 & .4 & .6 & \text { SF } 3 & 2.1 \mathrm{x} & 98 & 5\end{array}$

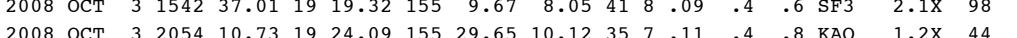

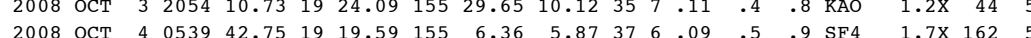

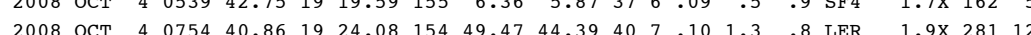

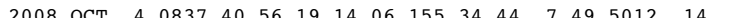

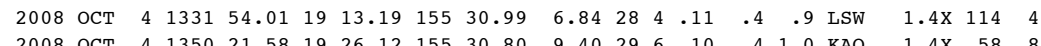

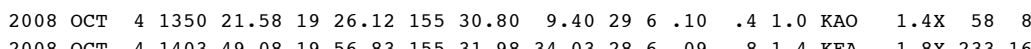

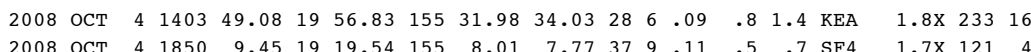

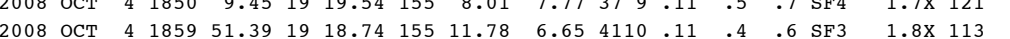

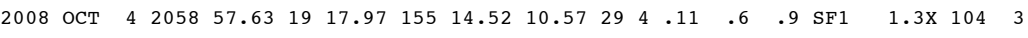

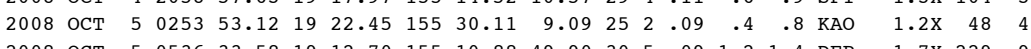

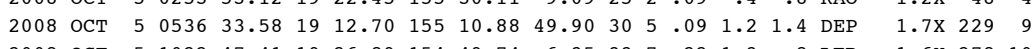

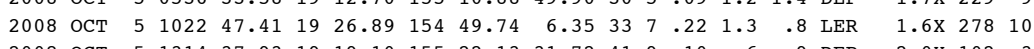

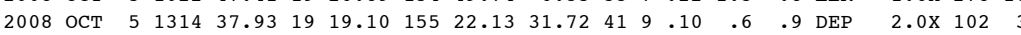

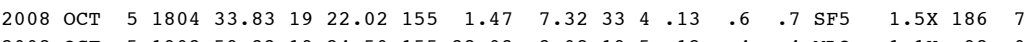

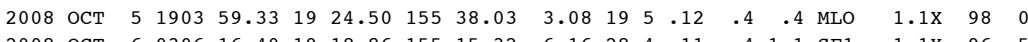

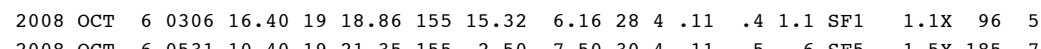
$\begin{array}{lllllllllllllllllll}2008 & \text { OCT } & 6 & 0531 & 10.40 & 19 & 21.35 & 155 & 2.50 & 7.50 & 30 & 4 & .11 & .5 & .6 & \text { SF5 } & 1.5 \times & 185 & 7\end{array}$ $\begin{array}{lllllllllllllllllll}2008 & \text { ОСТ } & 6 & 0643 & 5.11 & 19 & 20.24 & 155 & 5.69 & 8.91 & 33 & 7 & .09 & .5 & .4 & \text { SF } 4 & 1.4 \mathrm{X} & 164\end{array}$

$\begin{array}{llllllllllllllllll}2008 & \text { OCT } & 6 & 1821 & 39.00 & 19 & 23.77 & 155 & 15.78 & 2.97 & 20 & 5 & .07 & .3 & .3 & \text { SEC } & 1.7 \mathrm{X} & 102\end{array}$ $\begin{array}{llllllllllllllllll}2008 & \text { OCT } & 6 & 2257 & 31.07 & 19 & 17.85 & 155 & 14.14 & 6.91 & 37 & 4 & .11 & .5 & .6 & \text { SF2 } & 1.5 \mathrm{X} & 96\end{array}$

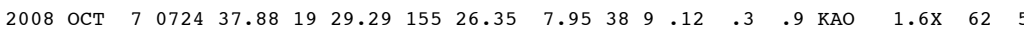

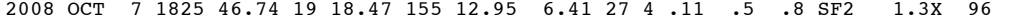

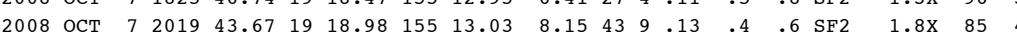

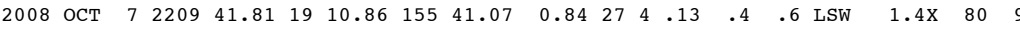
$\begin{array}{llllllllllllllllll}2008 & \text { OCT } & 8 & 0344 & 54.31 & 19 & 13.65 & 155 & 32.65 & 7.39 & 35 & 4 & .13 & .4 & .8 & \text { LSW } & 1.6 \mathrm{X} & 119\end{array}$ $\begin{array}{llllllllllllllllll}2008 & \text { OCT } & 8 & 0908 & 51.35 & 19 & 28.86 & 155 & 26.90 & 6.79 & 20 & 4 & .15 & .4 & 1.7 & \text { KAO } & 1.2 \mathrm{X} & 87\end{array}$ $\begin{array}{llllllllllllllllll}2008 & \text { OCT } & 8 & 1236 & 20.17 & 19 & 18.20 & 155 & 14.27 & 5.78 & 38 & 7 & .14 & .4 & .9 & \text { SF2 } & 1.6 \mathrm{X} & 90\end{array}$

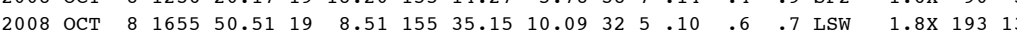

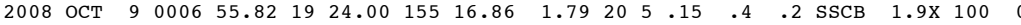

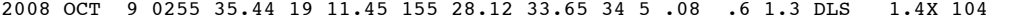

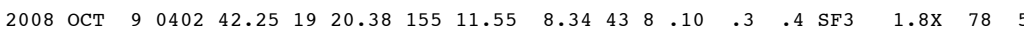

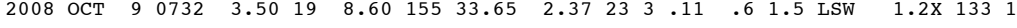

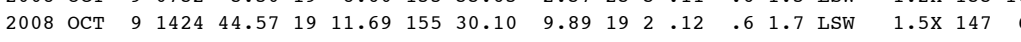

$\begin{array}{llllllllllllllllllll}2008 & \text { OCT } & 9 & 1451 & 36.32 & 19 & 10.34 & 155 & 28.35 & 35.11 & 40 & 8 & .09 & .6 & 1.2 & \text { DLS } & 1.7 \mathrm{x} & 81 & 2\end{array}$

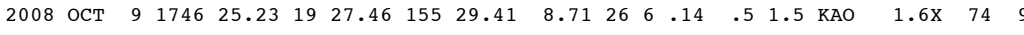

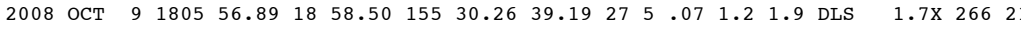

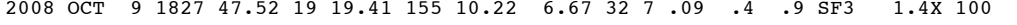

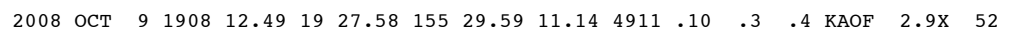


---ORIGIN TIME (HST)-- -LAT N-- --LON W-- DEPTH N N RMS ERH ERZ LOC PREF AZ MIN YEAR MON DA HRMN SEC DEG MIN DEG MIN KM RD S SEC KM KM REMKS MAG GAP DS $\begin{array}{llllllllllllllllll}2008 & \text { ост } & 9 & 1924 & 22.38 & 19 & 19.97 & 155 & 8.42 & 8.38 & 4711 & .11 & .4 & 4 & \text { SF } 4 & 2.5 \times & 110 & 5\end{array}$

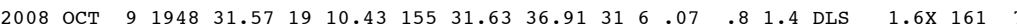

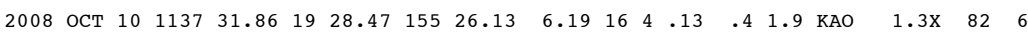

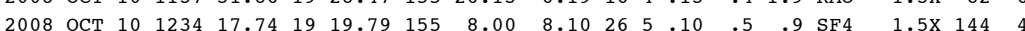

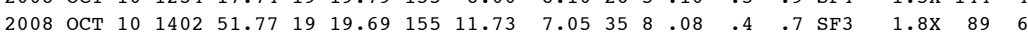

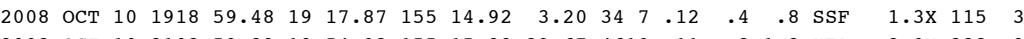
$\begin{array}{lllllllllllllllll}2008 & \text { ОСТ } 10 & 2102 & 59.39 & 19 & 54.02 & 155 & 15.33 & 39.67 & 4610 & .11 & .8 & 1.3 & \text { KEA } & 2.0 x & 228 & 9\end{array}$ $\begin{array}{lllllllllllllllllll}2008 & \text { ОСт } 10 & 2244 & 8.80 & 19 & 17.13 & 155 & 29.56 & 10.09 & 26 & 4 & .13 & .4 & .8 & \text { LSW } & 1.3 \mathrm{X} & 73 & 4\end{array}$

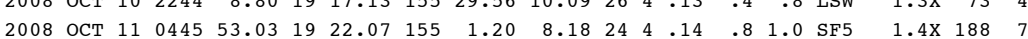

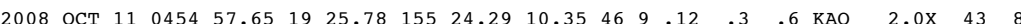

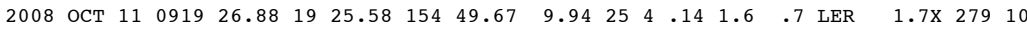

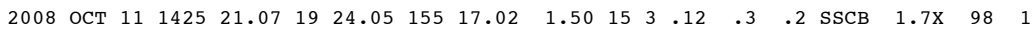

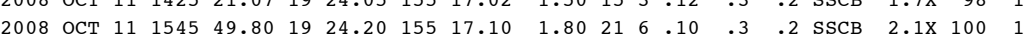

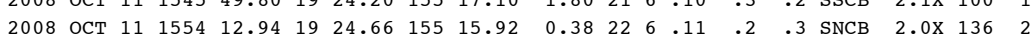

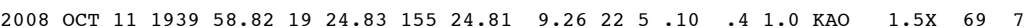

$\begin{array}{lllllllllllllllllll}2008 & \text { OCT } 11 & 2055 & 5.55 & 19 & 23.95 & 155 & 17.50 & 2.12 & 18 & 5 & .15 & .3 & .3 & \text { SSCB } & 1.9 \mathrm{X} & 79 & 1\end{array}$

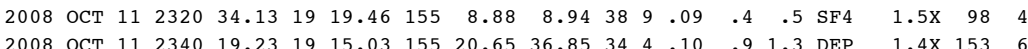

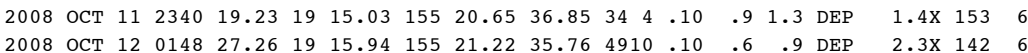

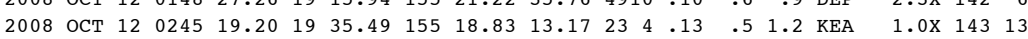

$\begin{array}{llllllllllllllllll}2008 & \text { ОСТ } 12 & 0335 & 28.61 & 19 & 12.15 & 155 & 30.67 & 36.69 & 29 & 3 & .07 & .7 & 1.4 & \text { DLS } & 1.4 \mathrm{X} & 81 & 5\end{array}$

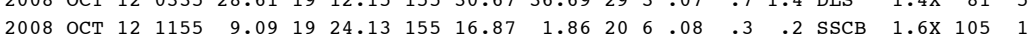

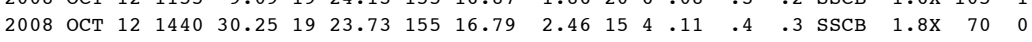
$\begin{array}{lllllllllllllllll}2008 & \text { ост } 12 & 1604 & 54.97 & 19 & 14.47 & 155 & 27.07 & 8.98 & 4710 & .14 & .4 & .6 & \text { LSW } & 2.2 \mathrm{x} & 97 & 5\end{array}$

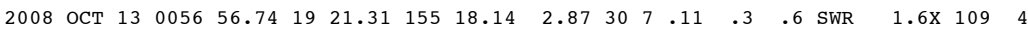

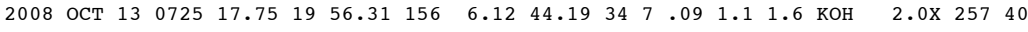
$\begin{array}{lllllllllllllllllll}2008 & \text { OCT } 13 & 1704 & 31.51 & 19 & 25.27 & 155 & 29.62 & 10.50 & 21 & 5 & .08 & .4 & 1.2 & \text { KAO } & 1.2 \mathrm{X} & 66 & 6\end{array}$

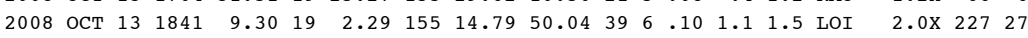

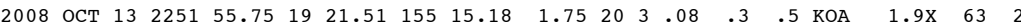

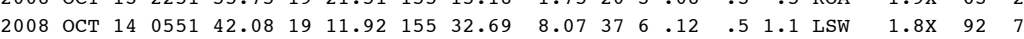

$\begin{array}{llllllllllllllllll}2008 & \text { ОСТ } 14 & 1024 & 12.97 & 19 & 21.25 & 155 & 6.44 & 8.65 & 4812 & .12 & .4 & .3 & \text { SF } 4 & 2.5 \times & 139 & 4\end{array}$ $\begin{array}{llllllllllllllllll}2008 & \text { OCT } 14 & 1225 & 27.68 & 19 & 24.00 & 155 & 16.97 & 1.79 & 16 & 5 & .09 & .3 & .2 & \text { SSCB } & 1.7 \mathrm{X} & 97 & 1\end{array}$ $\begin{array}{llllllllllllllllll}2008 & \text { ОСТ } 14 & 1226 & 26.07 & 19 & 24.29 & 155 & 16.48 & 1.14 & 26 & 6 & .14 & .2 & .2 & \text { SECB } & 2.2 \times & 122 & 1\end{array}$

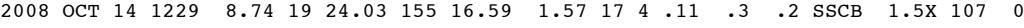

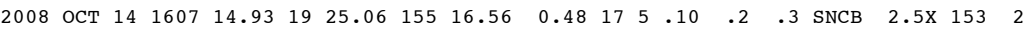

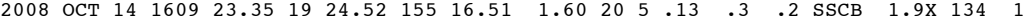

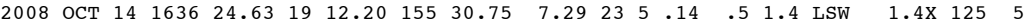
2008 OCT $14 \quad 1642 \quad 17.96 \quad 1914.01 \quad 156 \quad 20.02 \quad 12.08 \quad 18 \quad 3.13 \quad 7.711 .1$ DIS - 1.8X 30760

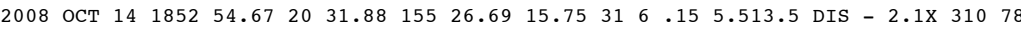

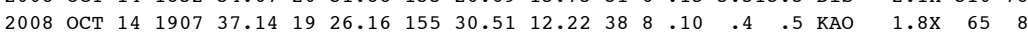

$\begin{array}{lllllllllllllllllll}2008 & \text { OCT } 14 & 2005 & 53.53 & 19 & 23.68 & 155 & 30.31 & 10.02 & 20 & 4 & .08 & .4 & 1.1 & \text { KAO } & 1.2 \mathrm{X} & 48 & 5\end{array}$

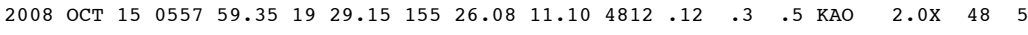

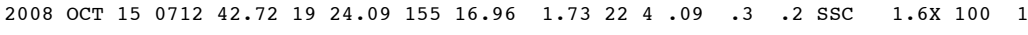

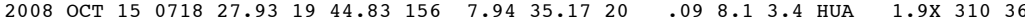

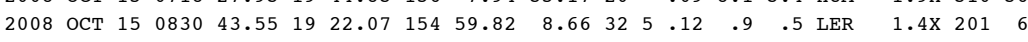

---ORIGIN TTME (HST)-- -LAT N-- --LON W-- DEPTH N N RMS ERH ERZ LOC PREF AZ MIN YEAR MON DA HRMN SEC DEG MIN DEG MIN KM RD S SEC KM KM REMRS MAG GAP DS

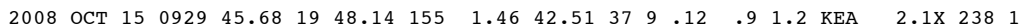
$\begin{array}{llllllllllllllllll}2008 & \text { ост } 15 & 1542 & 12.47 & 19 & 14.62 & 155 & 28.93 & 9.59 & 39 & 8 & .13 & .4 & .7 & \text { LSW } & 1.9 \mathrm{x} & 84 & 2\end{array}$

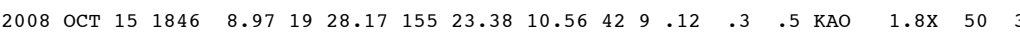

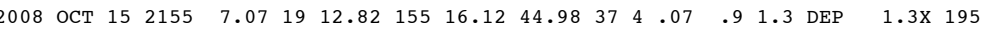

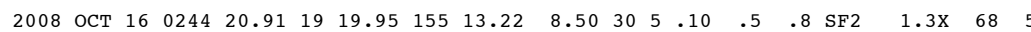

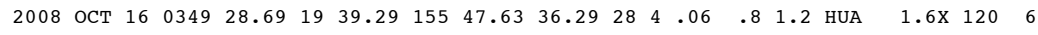

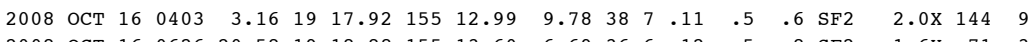

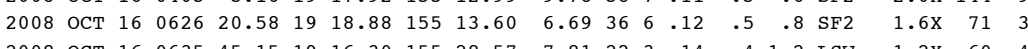

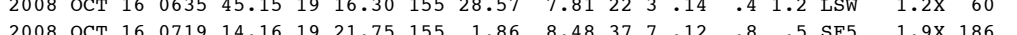

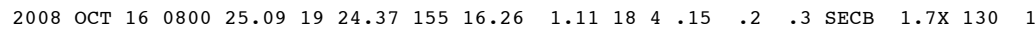

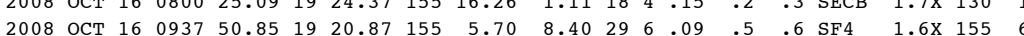

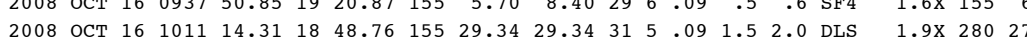

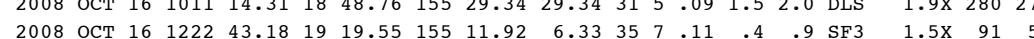

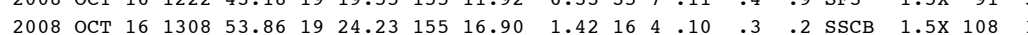

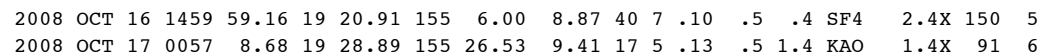

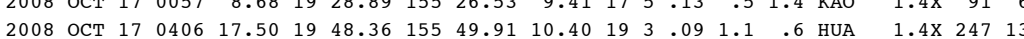

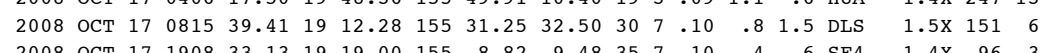

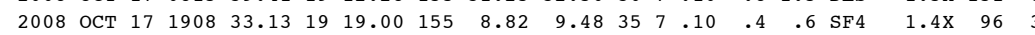

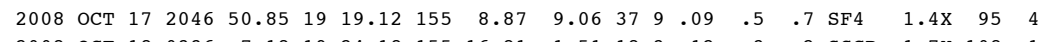

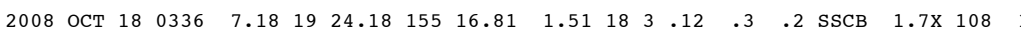

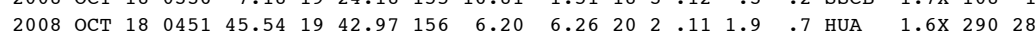
$\begin{array}{llllllllllllllll}2008 & \text { OCT } 18 & 0618 & 51.36 & 19 & 21.40 & 155 & 5.92 & 8.17 & 4310 & .11 & .4 & .4 & \text { SF } 4 & 2.0 \mathrm{X} & 145\end{array}$

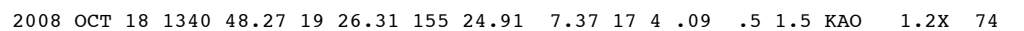

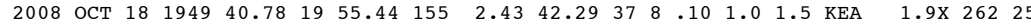

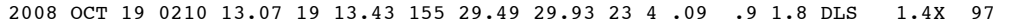

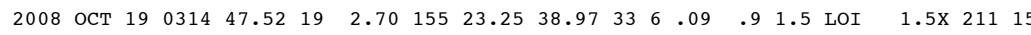

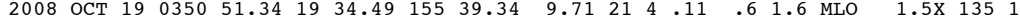

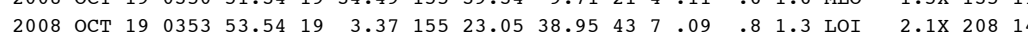

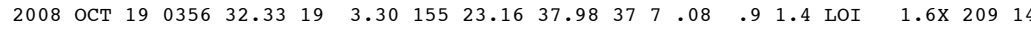

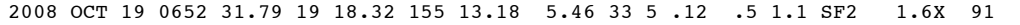

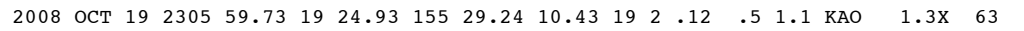
$\begin{array}{lllllllllllllllll}2008 & \text { OCT } 19 & 2355 & 9.61 & 19 & 28.53 & 155 & 15.12 & 33.51 & 25 & 4 & .11 & .9 & 1.3 & \text { DEP } & 1.4 \mathrm{X} & 125\end{array}$ $\begin{array}{llllllllllllll}2008 & \text { ост } 20 & 0109 & 9.27 & 19 & 46.49 & 156 & 1.24 & 6.07 & 18 & 2 & .09 & 1.4 & .7\end{array}$

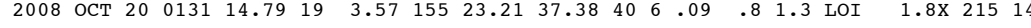

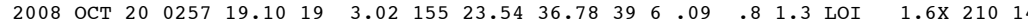

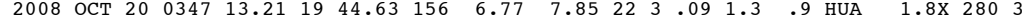

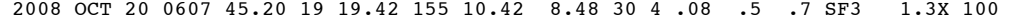

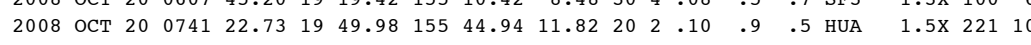

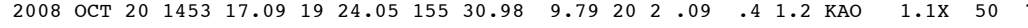

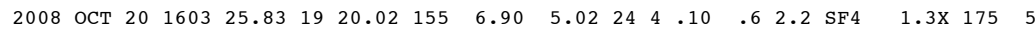

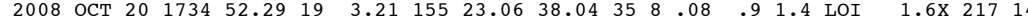

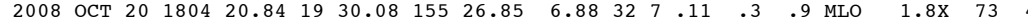

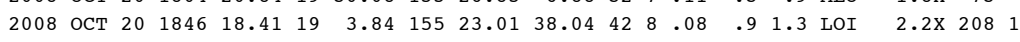


---ORIGIN TIME (HST)-- -LAT N-- --LON W-- DEPTH N N RMS ERH ERZ LOC PREF AZ MIN YEAR MON DA HRMN SEC DEG MIN DEG MIN KM RD S SEC KM KM REMKS MAG GAP DS

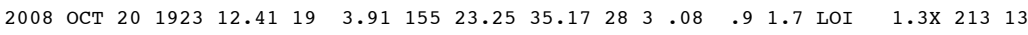

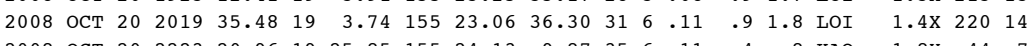
$\begin{array}{lllllllll} & \end{array}$

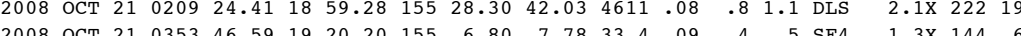

$\begin{array}{lllllllllllllllllll}2008 & \text { OCT } 21 & 0450 & 14.60 & 19 & 22.24 & 155 & 32.42 & 23.91 & 25 & 4 & .08 & .5 & 1.0 & \text { DML } & 1.4 \mathrm{X} & 90 & 5\end{array}$

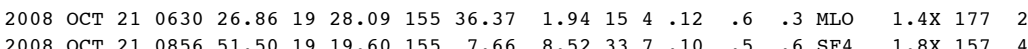

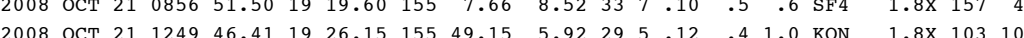

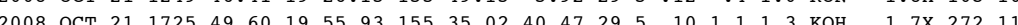

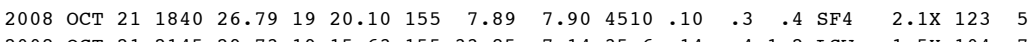

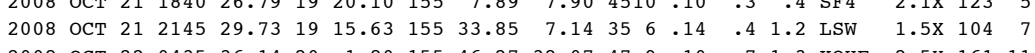

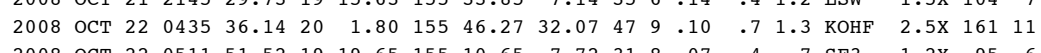

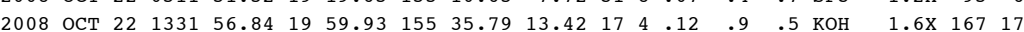
$\begin{array}{lllllllllllllllllll}2008 & \text { ОСТ } 22 & 1923 & 19.68 & 19 & 17.49 & 155 & 14.65 & 10.49 & 24 & 4 & .12 & .6 & 1.0 & \text { SF1 } & 1.3 \times & 126 & 2 \\ 2008 & \text { ОСТ } & 22 & 2039 & 33.19 & 19 & 27.73 & 155 & 27.65 & 10.80 & 17 & 4 & .13 & .6 & 1.5 & \text { KAO } & 1.4 \mathrm{X} & 84 & 8\end{array}$

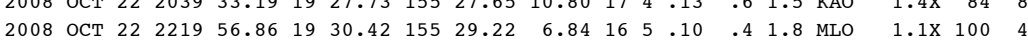

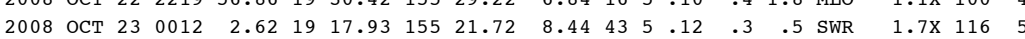

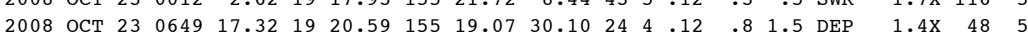

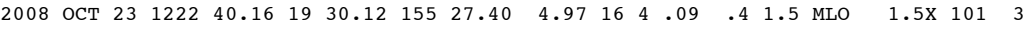
2008 ост $23142951.82 \quad 2022.62155 \quad 39.57 \quad 25.01 \quad 12 \quad .0812 .5 \quad 9.2$ кон $-1.9 \times 323 \quad 57$

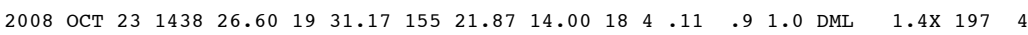

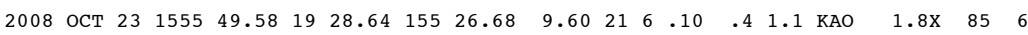
$\begin{array}{llllllllllllllllll}2008 & \text { ОСТ } 23 & 1742 & 13.74 & 19 & 21.17 & 155 & 0.41 & 6.91 & 33 & 8 & .11 & .6 & .6 & \text { SF5 } & 1.7 \mathrm{X} & 205 & 8\end{array}$

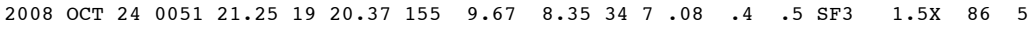

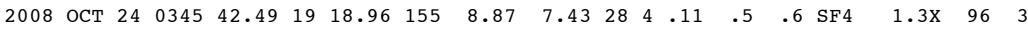

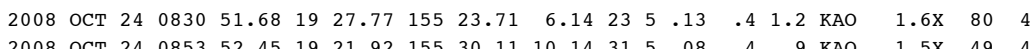

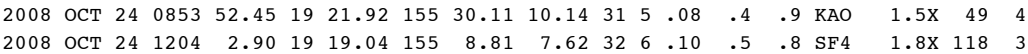

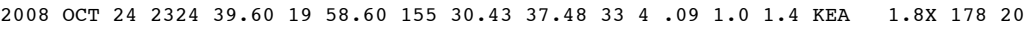

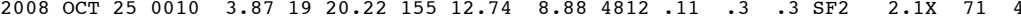

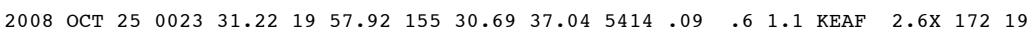

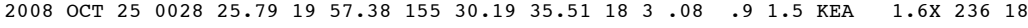

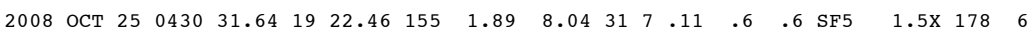

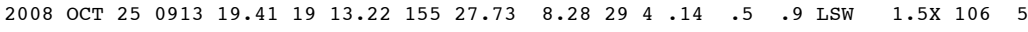

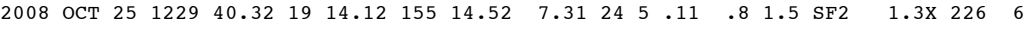

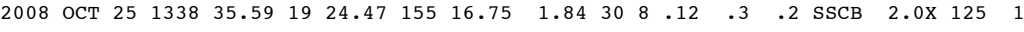

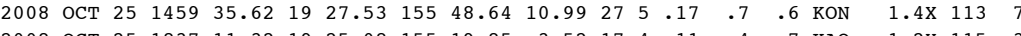

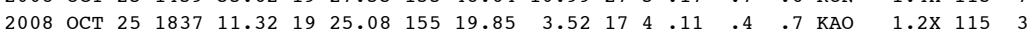

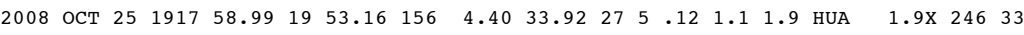

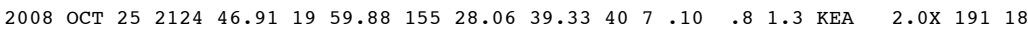

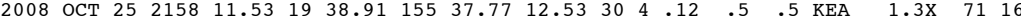

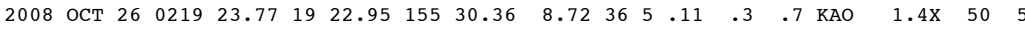

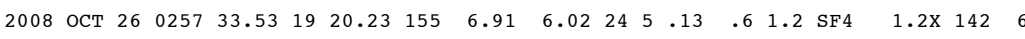

---ORIGIN TTME (HST)-- -LAT N-- --LON W-- DEPTH N N RMS ERH ERZ LOC PREF AZ MIN YEAR MON DA HRMN SEC DEG MIN DEG MIN KM RD S SEC KM KM REMKS MAG GAP DS

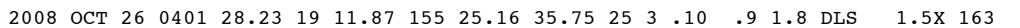

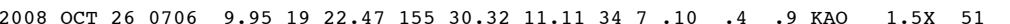

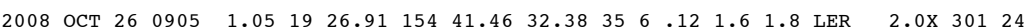

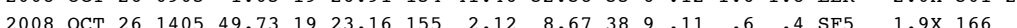

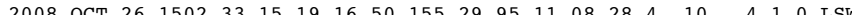

$\begin{array}{lllllllllllllllll}2008 & \text { OCT } 26 & 2329 & 6.47 & 19 & 30.27 & 155 & 26.75 & 4.17 & 17 & 4 & .12 & .4 & 1.3 & \text { MLO } & 1.5 \times & 100\end{array}$

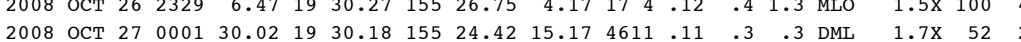

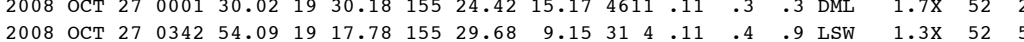

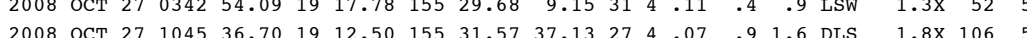

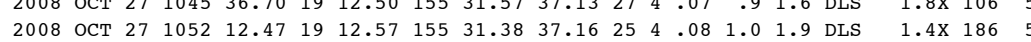

$\begin{array}{llllllllllllllllll}2008 & \text { OCT } 27 & 1423 & 48.59 & 19 & 24.07 & 155 & 17.06 & 2.35 & 18 & 4 & .13 & .4 & .3 & \text { SSCB } & 2.0 x & 97 & 1\end{array}$

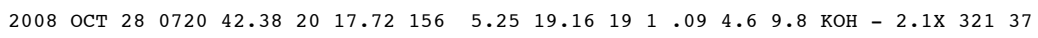

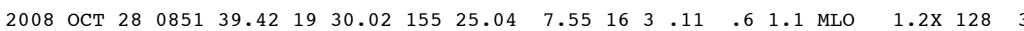
$\begin{array}{llllllllllllll}2008 & \text { OCT } 28 & 1205 & 21.86 & 19 & 29.21 & 155 & 27.00 & 8.23 & 31 & 4 & .13 & .4 & 1.1 \\ \text { KAO } & 1.6 \mathrm{x} & 74 & 5\end{array}$ $\begin{array}{lllllllllllllllllll}2008 & \text { OCT } & 28 & 1815 & 48.95 & 19 & 29.06 & 155 & 27.13 & 5.59 & 18 & 4 & .09 & .4 & 2.2 & \text { KAO } & 1.6 \mathrm{X} & 87 & 6\end{array}$

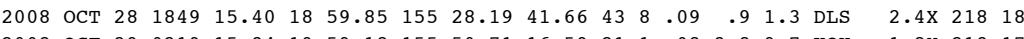

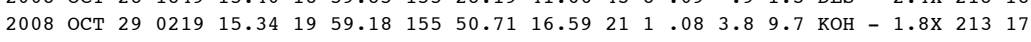

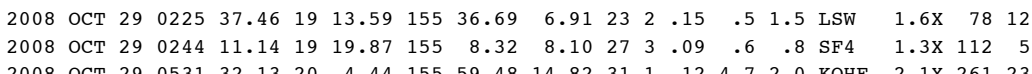

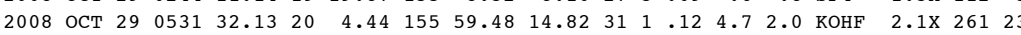

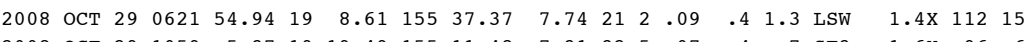

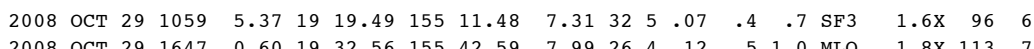

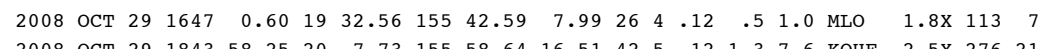

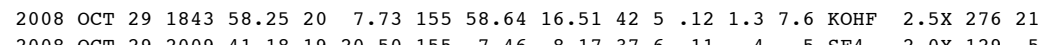

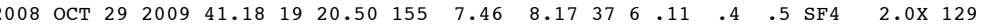

$\begin{array}{llllllllllllllllll}2008 & \text { OCT } 29 & 2240 & 46.68 & 19 & 24.75 & 155 & 20.12 & 6.83 & 26 & 7 & .10 & .4 & .9 & \text { KAO } & 1.7 \mathrm{X} & 104 & 2\end{array}$

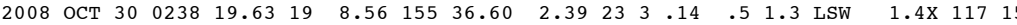

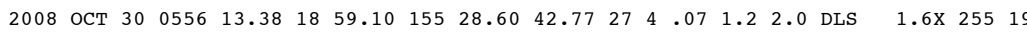

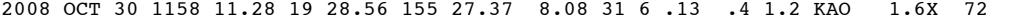

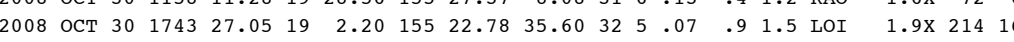

$\begin{array}{llllllllllllllllll}2008 & \text { ОСТ } 30 & 2139 & 53.50 & 19 & 21.93 & 155 & 28.10 & 9.01 & 38 & 7 & .11 & .4 & .6 & \text { KAO } & 2.0 x & 60 & 1\end{array}$

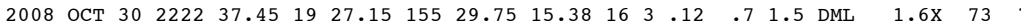

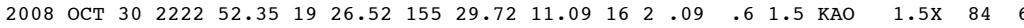
$\begin{array}{llllllllllllllllll}2008 & \text { OCT } 31 & 0723 & 30.17 & 18 & 59.20 & 155 & 28.55 & 42.87 & 22 & 3 & .07 & 1.4 & 2.1 & \text { DLS } & 1.4 \times & 264 & 19\end{array}$

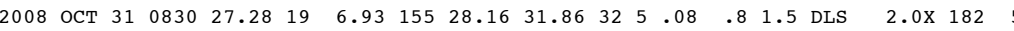

$\begin{array}{llllllllllllllllll}2008 & \text { ОСТ } 31 & 0850 & 58.06 & 19 & 34.26 & 155 & 43.53 & 9.05 & 26 & 4 & .11 & .5 & 1.2 & \text { KON } & 2.0 \mathrm{X} & 92 & 8\end{array}$

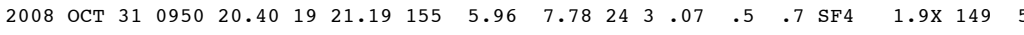

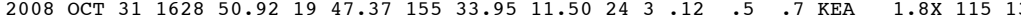

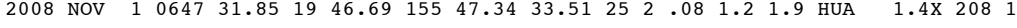

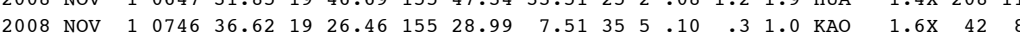

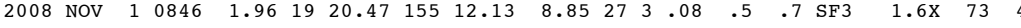

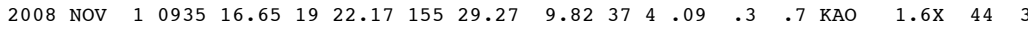

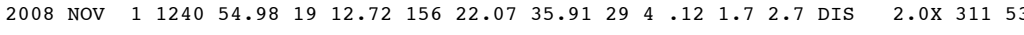

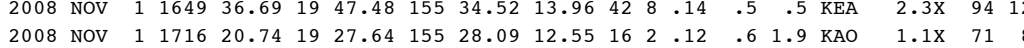


---ORIGIN TIME (HST)-- -LAT N-- --LON W-- DEPTH N N RMS ERH ERZ LOC PREF AZ MIN YEAR MON DA HRMN SEC DEG MIN DEG MIN KM RD S SEC KM KM REMKS MAG GAP DS 2008 NOV $20 \begin{array}{lllllllllllllllll} & 0701 & 48.68 & 19 & 19.15 & 155 & 8.50 & 9.54 & 46 & 9 & .10 & .5 & .4 & \text { SF4 } & 2.7 X & 106 & 3\end{array}$

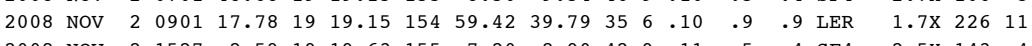
$\begin{array}{lllllllllll}2008 & & 0\end{array}$

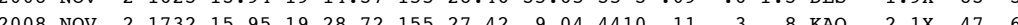

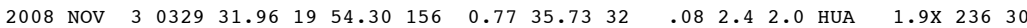

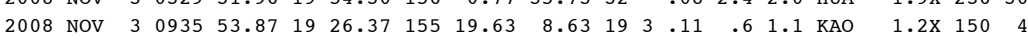

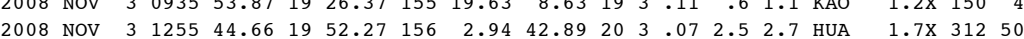
2008 NOV $3 \begin{array}{llllllllllllllll}1457 & 38.15 & 19 & 31.13 & 155 & 28.23 & 4.87 & 13 & 3 & .09 & .4 & 1.1 & \text { MLO } & 1.4 \times & 95 & 2\end{array}$

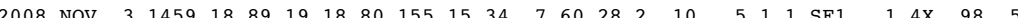

2008 NOV $4 \quad \begin{array}{llllllllllllllllll}0 & 27.43 & 19 & 18.24 & 154 & 58.42 & 42.51 & 31 & 2 & .10 & 1.6 & 1.5 & \text { LER } & 1.9 \times & 242 & 13\end{array}$ 2008 Nov $4 \begin{array}{lllllllllllllllll}0 & 0403 & 53.67 & 19 & 25.27 & 155 & 26.67 & 1.86 & 15 & 3 & .08 & .4 & 1.2 & \text { KAO } & 1.0 \mathrm{X} & 68 & 5\end{array}$

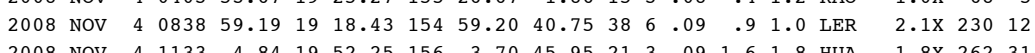

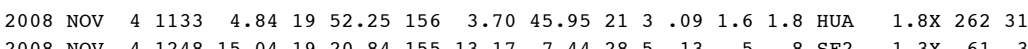

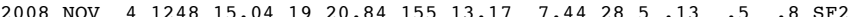

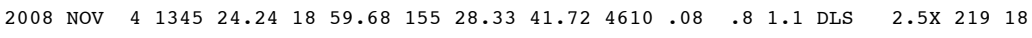

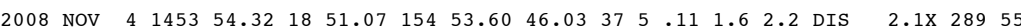

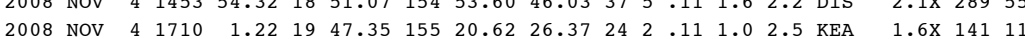
2008 Nov $4 \begin{array}{llllllllllllllll}4746 & 36.32 & 19 & 21.68 & 155 & 30.04 & 9.52 & 42 & 8 & .10 & .4 & .5 & \text { KAO } & 2.0 \times & 50 & 5\end{array}$ 2008 NOV $4 \begin{array}{llllllllllllllllll}1818 & 7.17 & 19 & 14.87 & 155 & 16.06 & 30.06 & 39 & 7 & .10 & .7 & .8 & \text { DEP } & 2.2 \times & 194 & 6\end{array}$

$\begin{array}{lllllllllllllllllll}2008 \text { NOV } & 4 & 2343 & 14.83 & 19 & 19.42 & 155 & 13.61 & 8.10 & 38 & 5 & .13 & .5 & .6 & \text { SF2 } & 1.9 \mathrm{X} & 67 & 4 \\ 2008 & & \end{array}$

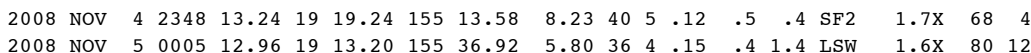

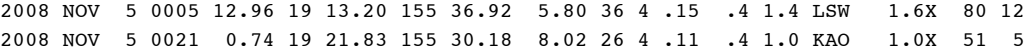

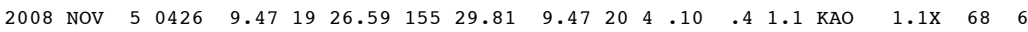

2008 NOV $5 \begin{array}{lllllllllllllllll}0 & 0913 & 42.18 & 19 & 14.51 & 155 & 28.84 & 8.23 & 27 & 3 & .13 & .4 & 1.0 & \text { LSW } & 1.1 \mathrm{x} & 83 & 2\end{array}$

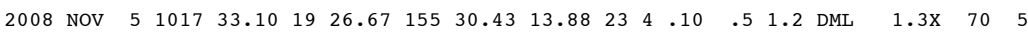
2008 Nov $5 \begin{array}{llllllllllllllll}1036 & 35.82 & 19 & 25.93 & 155 & 24.10 & 7.24 & 20 & 5 & .13 & .4 & 1.6 & \text { KAO } & 1.3 \mathrm{X} & 83 & 7\end{array}$ 2008 NOV $5 \begin{array}{lllllllllllllllll}5 & 1638 & 50.75 & 19 & 21.98 & 155 & 4.69 & 7.04 & 25 & 4 & .13 & .7 & 1.0 & \text { SF5 } & 1.6 \times & 154 & 4\end{array}$ 2008 NOV $5 \begin{array}{llllllllllllllllll}5 & 1743 & 8.83 & 19 & 24.73 & 155 & 36.87 & 2.56 & 15 & 3 & .10 & .4 & .4 & \text { MLO } & 1.0 x & 94 & 2\end{array}$

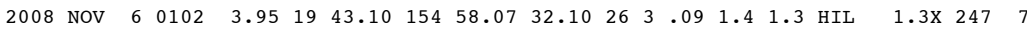
2008 NOV $6 \begin{array}{lllllllllllllllll}0 & 0334 & 9.24 & 19 & 25.08 & 155 & 24.04 & 8.75 & 29 & 3 & .12 & .4 & 1.0 & \text { KAO } & 1.1 \mathrm{x} & 78 & 8\end{array}$

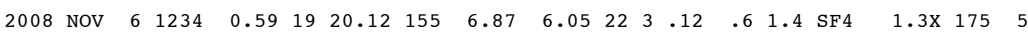

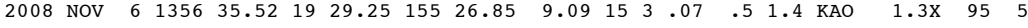
2008 Nov $6 \begin{array}{llllllllllllllll}1922 & 28.60 & 19 & 21.51 & 155 & 4.75 & 8.79 & 37 & 9 & .11 & .6 & .4 & \text { SF5 } & 2.1 \times & 160 & 5\end{array}$

2008 NOV $6 \begin{array}{lllllllllllllllll}6 & 2226 & 9.87 & 19 & 33.53 & 155 & 43.80 & 12.28 & 24 & 4 & .11 & .6 & .6 & \text { Kon } & 1.4 \times & 108 & 7\end{array}$

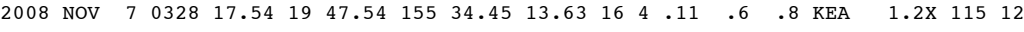
2008 NOV $7 \begin{array}{lllllllllllllllll} & 0639 & 23.42 & 19 & 16.94 & 155 & 30.27 & 9.90 & 35 & 2 & .12 & .4 & .6 & \text { LSW } & 1.8 \mathrm{x} & 68 & 3\end{array}$ 2008 Nov $7 \begin{array}{lllllllllllllllll}7 & 0641 & 51.13 & 19 & 16.78 & 155 & 30.44 & 12.31 & 34 & 4 & .10 & .4 & .6 & \text { LSW } & 1.9 x & 74 & 3\end{array}$

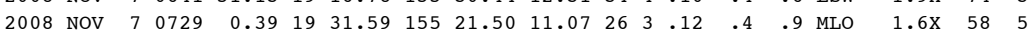

2008 NOV $7 \begin{array}{llllllllllllllll}1052 & 34.64 & 19 & 20.15 & 155 & 21.15 & 32.15 & 5011 & .10 & .5 & .8 & \text { DEPF } & 2.8 \mathrm{X} & 74 & 4\end{array}$

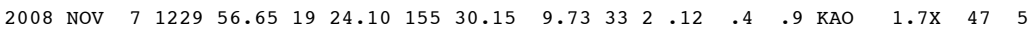

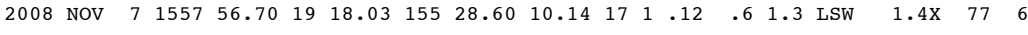

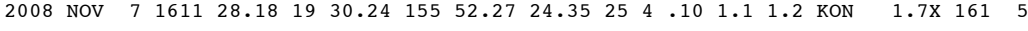
2008 Nov $\quad \begin{array}{llllllllllllllll}1814 & 57.63 & 19 & 18.41 & 156 & 18.49 & 38.35 & 46 & 8 & .10 & 1.3 & 1.6 & \text { KON } & 2.8 \times & 273 & 46\end{array}$
---ORTGIN TIME (HST)-- -LAT N-- --LON W-- DEPTH N N RMS ERH ERZ LOC PREF AZ MTM YEAR MON DA HRMN SEC DEG MIN DEG MIN KM RD S SEC KM KM REMKS MAG GAP DS

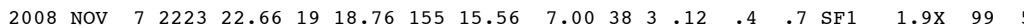

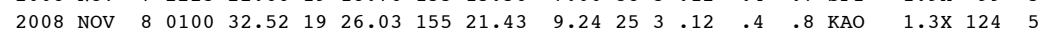

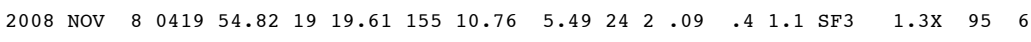
$\begin{array}{llllllllllll}7.69 & 19 & 11.95 & 155 & 26.07 & 45.67 & 24 & 1 & .09 & 1.2 & 1.9 & \text { DLS }\end{array}$

$\begin{array}{lllllllllllllllllllll}2008 \text { Nov } 8 & 1643 & 7.72 & 19 & 20.27 & 155 & 11.74 & 5.74 & 34 & 3 & .13 & .4 & .8 & \mathrm{SF} 3 & 1.7 \mathrm{X} & 78 & 5\end{array}$

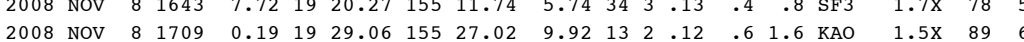

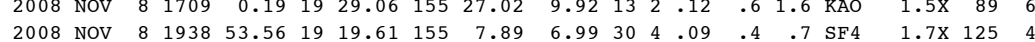
2008 NOV $8 \begin{array}{llllllllllllllllll}8 & 2358 & 10.83 & 19 & 19.17 & 154 & 59.28 & 39.46 & 27 & 3 & .10 & 1.2 & 1.4 & \text { LER } & 2.1 \times & 227 & 11\end{array}$

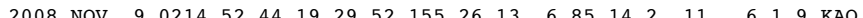

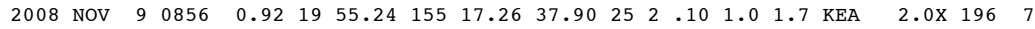

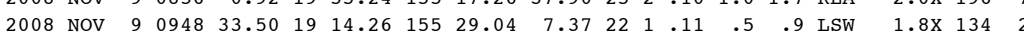

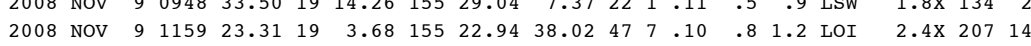
2008 Nov $9 \begin{array}{lllllllllllllllll}1506 & 49.61 & 19 & 10.66 & 155 & 37.30 & 0.02 & 30 & 3 & .18 & .5 & .6 & \text { LSW \# } 1.8 \mathrm{x} & 97 & 15\end{array}$

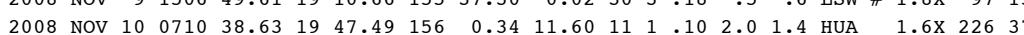

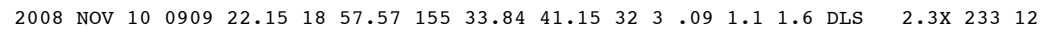

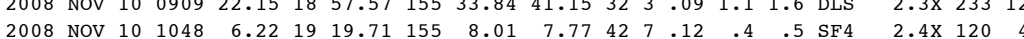

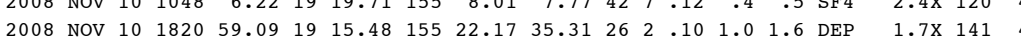
2008 NOV $10 \begin{array}{lllllllllllll}10 & 2213 & 22.82 & 19 & 21.84 & 155 & 25.73 & 9.73 & 24 & 1 & .11 & .4 & .8 \\ \text { KAO } & 1.3 \times & 55\end{array}$

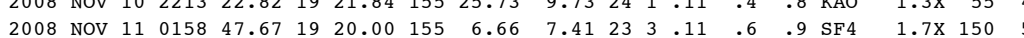

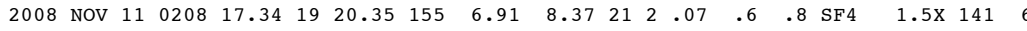

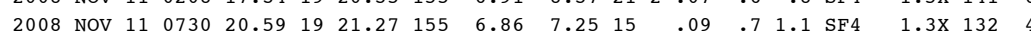

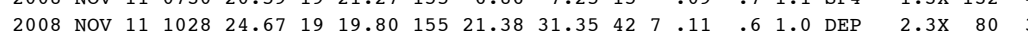

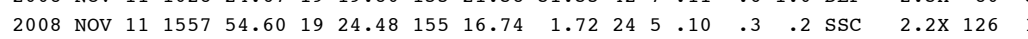

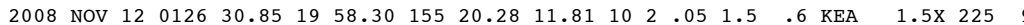

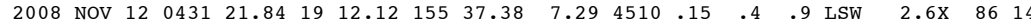

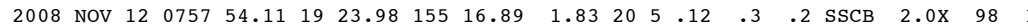

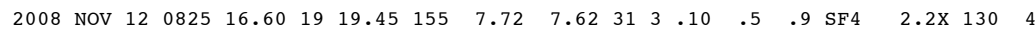

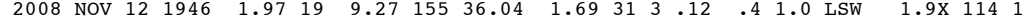

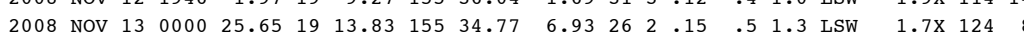

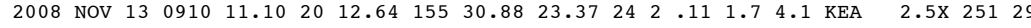

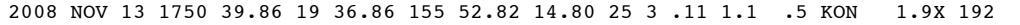

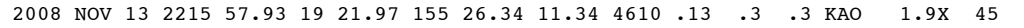

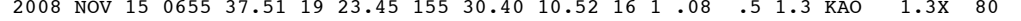

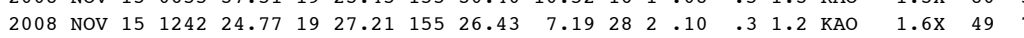

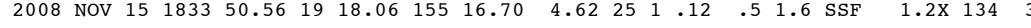

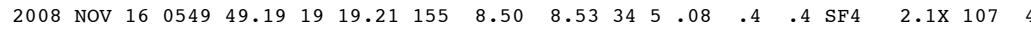

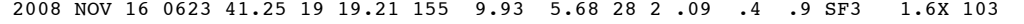

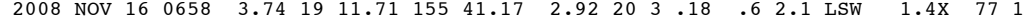

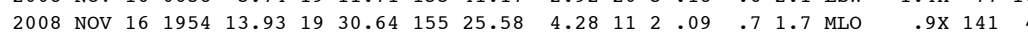

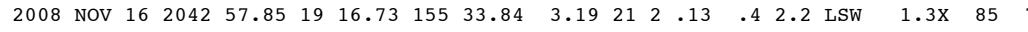

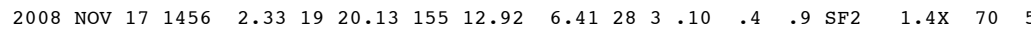

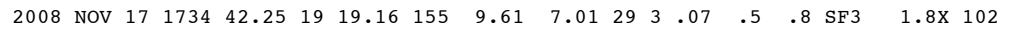

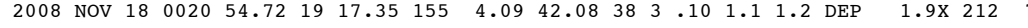

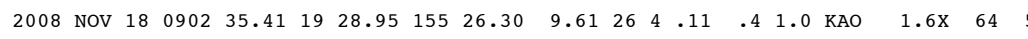


---ORIGIN TIME (HST)-- -LAT N-- --LON W-- DEPTH N N RMS ERH ERZ LOC PREF AZ MIN YEAR MON DA HRMN SEC DEG MIN DEG MIN KM RD S SEC KM KM REMKS MAG GAP DS 2008 NOV $18 \quad 120620.96 \quad 1929.12 \quad 155 \quad 27.32 \quad 7.67 \quad 132.14 \quad 6 \quad 2.0$ KAO 2008 NOV $18 \quad 1426 \quad 18.691933 .23 \quad 155 \quad 18.37 \quad 28.16 \quad 12 \quad 2 \quad .11 \quad 2.8 \quad 2.0$ KAD

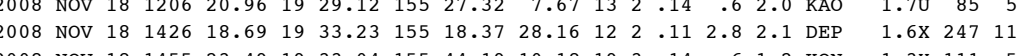
$\begin{array}{llllll} & \end{array}$

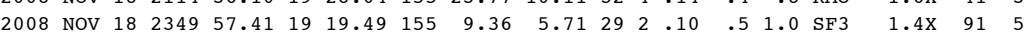

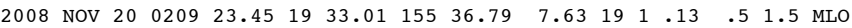

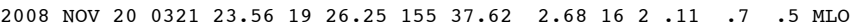
2008 NOV $20 \quad 2225 \quad 11.56 \quad 1926.27 \quad \begin{array}{lllllllll}15 & 56.10 & 6.63 & 22 & 2 & 11 & 1.7 & .5 & \text { MLO }\end{array}$

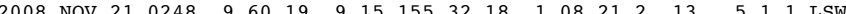

$.6 \mathrm{X} \quad 53 \quad 4$ $1.5 \times \quad 94 \quad 7$ $\begin{array}{lll}1.5 \times & 181 & 3 \\ 1.5 \times & 208 & 3 \\ 1.5 \times 132 & 13\end{array}$ $1.5 \times 132 \quad 8$

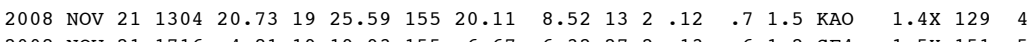

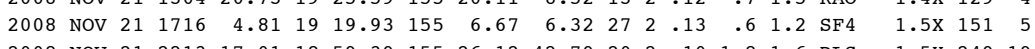

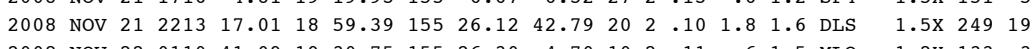

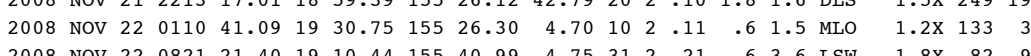

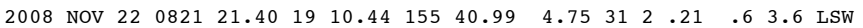

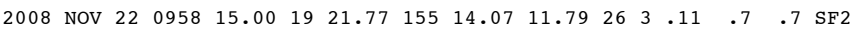

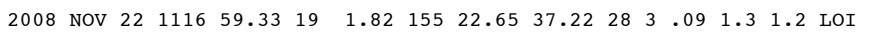

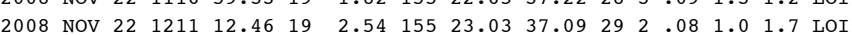

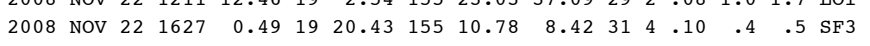
2008 NOV $22 \quad \begin{array}{lllllllllllllll}1853 & 35.60 & 19 & 10.84 & 155 & 19.92 & 47.08 & 23 & 1 & .11 & 1.3 & 2.6 & \text { DEP }\end{array}$

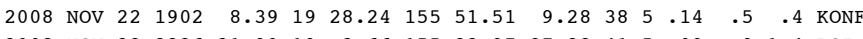

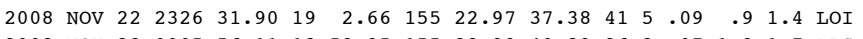

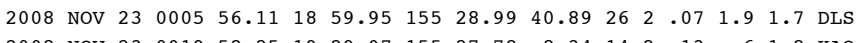

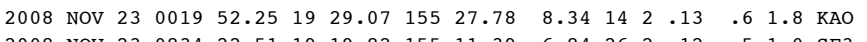

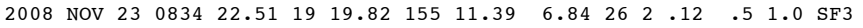

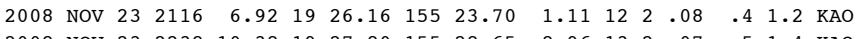

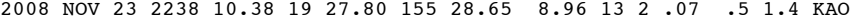

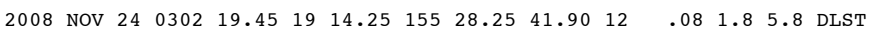

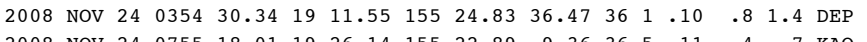

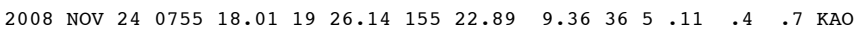

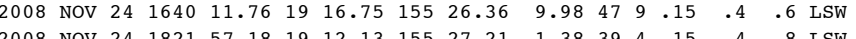

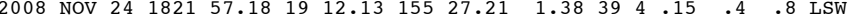

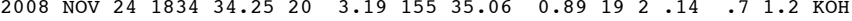

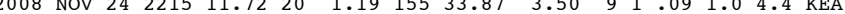

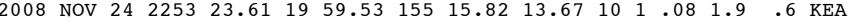

$\begin{array}{lll}.7 \times \quad 97 \quad 2 \\ .7 \times & 216 & 17\end{array}$ $1.7 \times \quad 216 \quad 17$ $.6 \times 21215$ $\begin{array}{rrr}.1 \times & 79 & 4 \\ 2 \times & 200 & 10\end{array}$ $2.2 \times 104 \quad 7$ $.4 \times 21215$ $2.2 \times \quad 237 \quad 18$ $\begin{array}{lll}.6 \mathrm{x} & 87 & 5 \\ .0 \mathrm{x} & 89 & 6\end{array}$ $\begin{array}{lll}.1 \times & 92 & 7\end{array}$ $\begin{array}{rrr}1 \times & 97 & 8 \\ & 121 & 4\end{array}$ $1.8 \times \quad 47 \quad 7$

$2.5 \times \quad 55 \quad 6$ $2.0 \times 120 \quad 5$ $.1 \times 19022$ $\begin{array}{lll}.1 \times & 182 & 20 \\ .4 \times & 251 & 14\end{array}$

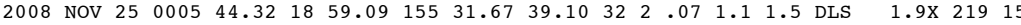

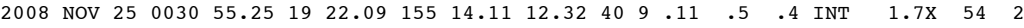

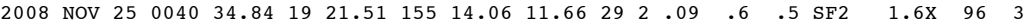

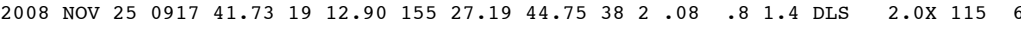

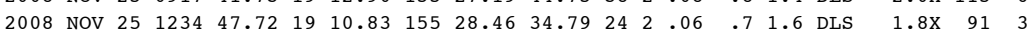

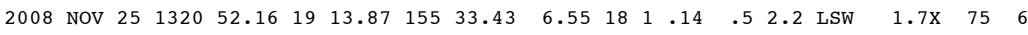

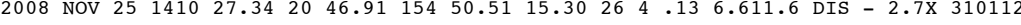

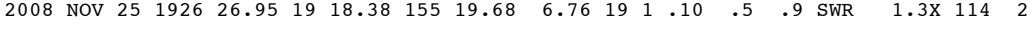

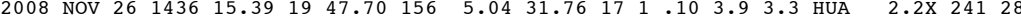

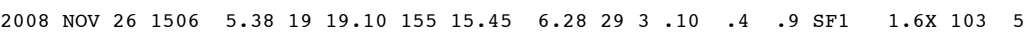

---ORIGIN TIME (HST)-- -LAT N-- --LON W-- DEPTH N N RMS ERH ERZ LOC PREF AZ MIN YEAR MON DA HRMN SEC DEG MIN DEG MIN KM RD S SEC KM KM REMKS MAG GAP DS

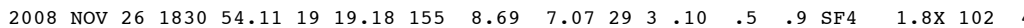

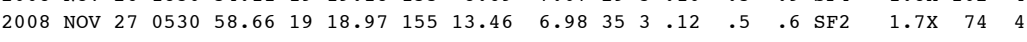
2008 NOV $27 \quad \begin{array}{llllllllllllllll}1353 & 33.25 & 19 & 30.65 & 155 & 24.01 & 14.13 & 18 & 3 & .13 & .6 & .9 & \text { DML } & 1.3 \mathrm{X} & 131 & 2\end{array}$ $\begin{array}{llllllllllllllllll}2008 \text { NOV } 27 & 1532 & 27.22 & 19 & 24.69 & 155 & 29.85 & 8.58 & 21 & 1 & .11 & .4 & 1.1 & \text { KAO } & 1.3 \times & 66 & 6\end{array}$ 2008 Nov $27 \begin{array}{lllllllllllllll}1608 & 29.14 & 19 & 18.93 & 155 & 13.55 & 7.27 & 38 & 7 & .11 & .4 & .5 & \text { SF2 } & 2.0 \mathrm{x} & 71\end{array}$

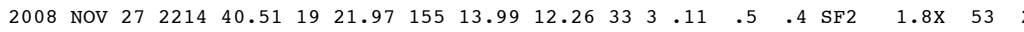

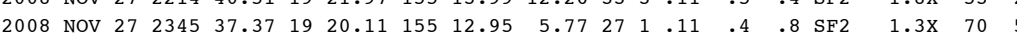

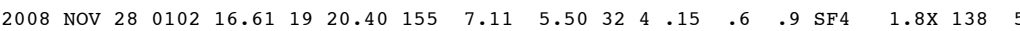

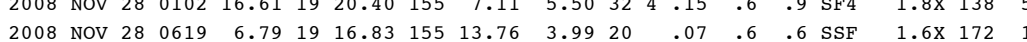

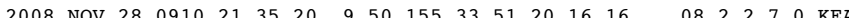

$\begin{array}{lllllllllllllllll}2008 & \text { NOV } 28 & 1930 & 3.85 & 19 & 15.19 & 155 & 21.98 & 3.71 & 26 & .15 & .6 & 1.5 & \text { SWR } & 1.3 \times & 145 & 4\end{array}$

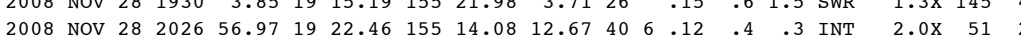

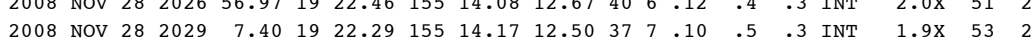

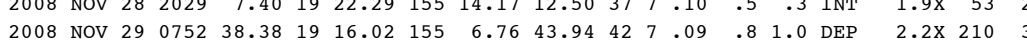

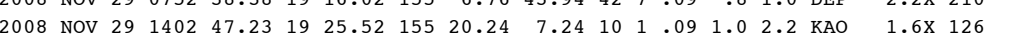

2008 NOV $29 \begin{array}{llllllllllllllllll}1924 & 48.60 & 19 & 23.26 & 155 & 30.44 & 9.63 & 34 & 2 & .12 & .4 & .8 & \text { KAO } & 1.5 \mathrm{X} & 50 & 5\end{array}$

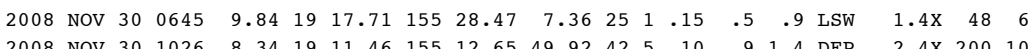

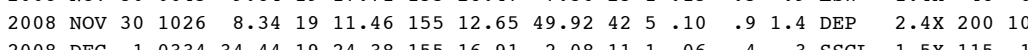

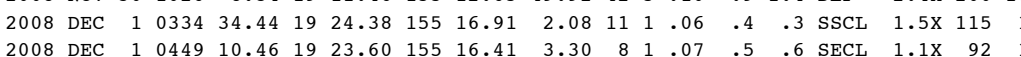

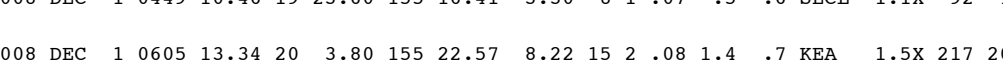

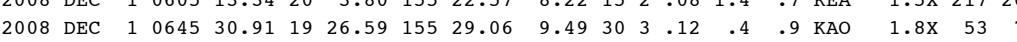

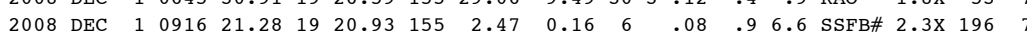

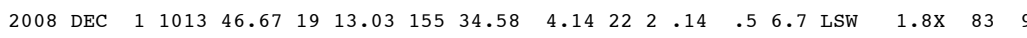

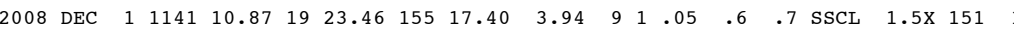

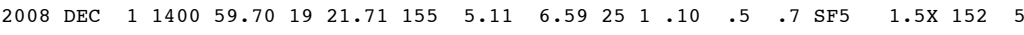

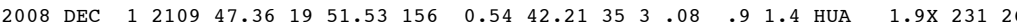
$\begin{array}{llllllllllllllllll}2008 & \text { DEC } & 1 & 2131 & 51.07 & 19 & 24.16 & 155 & 17.97 & 9.28 & 8 & 1 & .04 & 1.5 & 2.4 & \text { INTL } & 1.6 \times & 124\end{array}$ $\begin{array}{llllllllllllllllll}2008 & \text { DEC } & 1 & 2135 & 29.70 & 19 & 24.29 & 15 & 16.83 & 4.56 & 11 & 1 & .06 & .6 & .8 & \text { SSCL } & 1.6 \mathrm{X} & 113\end{array}$

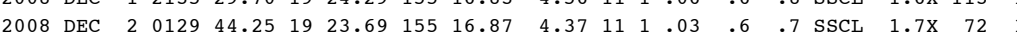

$\begin{array}{lllllllllllllllllll}2008 & \text { DEC } & 2 & 0307 & 3.26 & 18 & 50.59 & 155 & 22.91 & 24.36 & 29 & 2 & .07 & 1.8 & 3.3 & \text { LOI } & 1.8 \times & 262 & 34\end{array}$

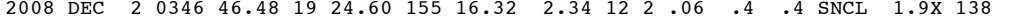
$\begin{array}{llllllllllllllllll}2008 & \text { DEC } & 2 & 0350 & 14.77 & 19 & 23.77 & 155 & 16.68 & 2.00 & 11 & 1 & .10 & .4 & .3 & \text { SSCL } & 2.0 \mathrm{X} & 62\end{array}$

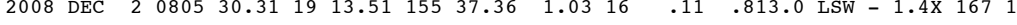
$\begin{array}{lllllllllllllllll}2008 & \text { DEC } & 2 & 0817 & 9.22 & 18 & 51.34 & 155 & 23.24 & 25.94 & 34 & 3 & .08 & 1.5 & 2.8 & \text { LOI } & 2.3 \times \\ 260 & 33\end{array}$

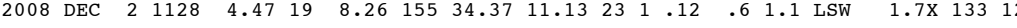

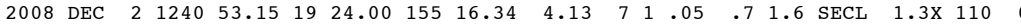
$\begin{array}{llllllllllllllllll}2008 & \text { DEC } & 2 & 1346 & 33.36 & 19 & 14.75 & 155 & 8.10 & 40.77 & 27 & 2 & .09 & 1.5 & 1.8 & \text { DEP } & 1.5 \times & 244\end{array}$

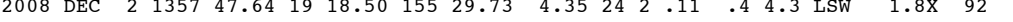

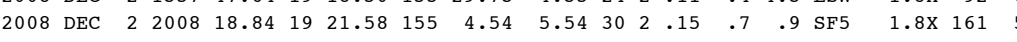

$\begin{array}{llllllllllllllllll}2008 & \text { DEC } & 2 & 2201 & 24.66 & 19 & 24.94 & 155 & 16.84 & 3.51 & 10 & 2 & .04 & .5 & .6 & \text { SNCL } & 2.1 \mathrm{x} & 153\end{array}$

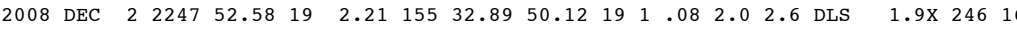

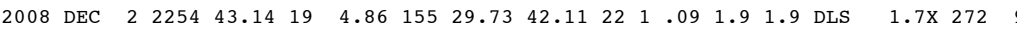

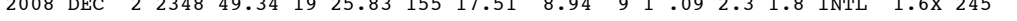

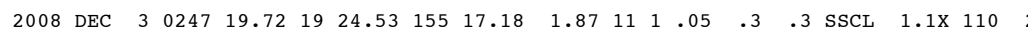


---ORIGIN TIME (HST)-- -LAT N-- --LON W-- DEPTH N N RMS ERH ERZ LOC PREF AZ MIN YEAR MON DA HRMN SEC DEG MIN DEG MIN KM RD S SEC KM KM REMKS MAG GAP DS

$\begin{array}{lllllllllllllllllll}2008 & \text { DEC } & 3 & 0512 & 33.07 & 19 & 25.64 & 155 & 17.60 & 4.34 & 10 & 1 & .06 & 1.3 & .5 & \text { SNCL } & 1.7 \times & 241 & 0\end{array}$

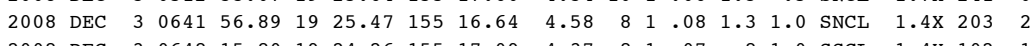

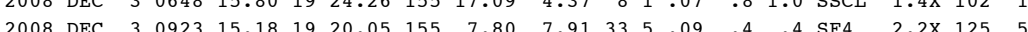

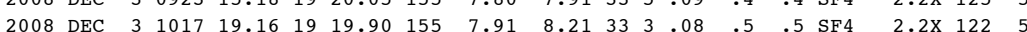

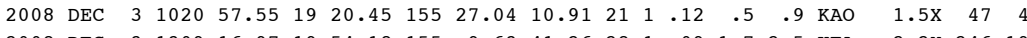

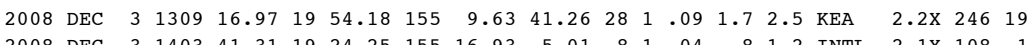

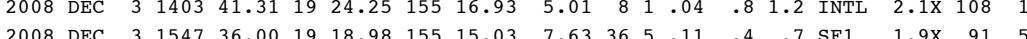

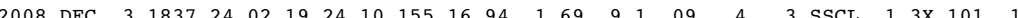

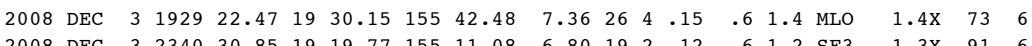

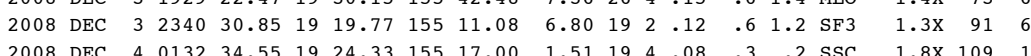

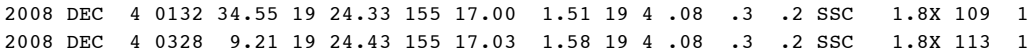

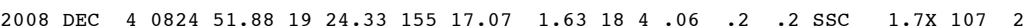

$\begin{array}{lllllllllllllllllll}2008 & \text { DEC } & 4 & 0843 & 1.12 & 19 & 24.18 & 155 & 16.91 & 1.91 & 7 & 1 & .06 & .7 & .5 & \text { SSCL } & 1.2 \times & 149 & 1\end{array}$

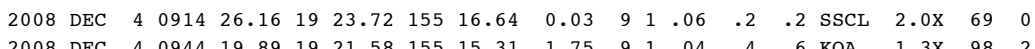

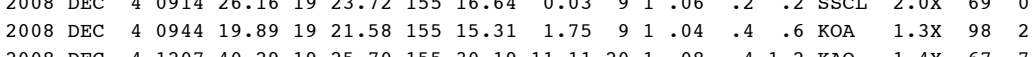

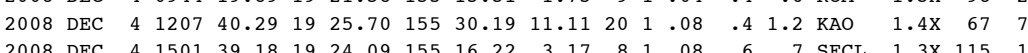
$\begin{array}{llllllllllllllllll}2008 & \text { DEC } & 4 & 1501 & 39.18 & 19 & 24.09 & 155 & 16.22 & 3.17 & 8 & 1 & .08 & .6 & .7 & \text { SECL } & 1.3 \mathrm{XX} & 115 \\ & & & & & & & & & & & & & & & & \end{array}$

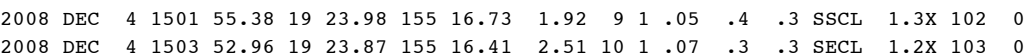

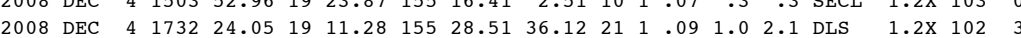

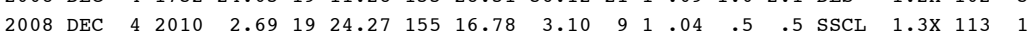

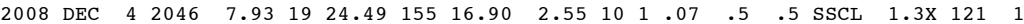

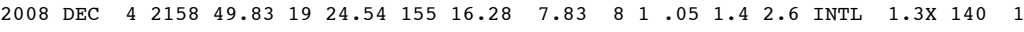

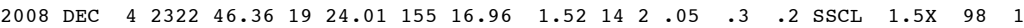

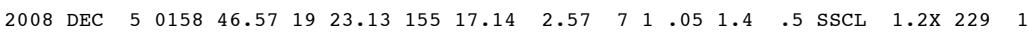
$\begin{array}{lllllllllllllllllll}2008 & \text { DEC } & 5 & 0206 & 24.08 & 19 & 24.04 & 155 & 17.10 & 2.15 & 10 & 1 & .07 & .4 & .3 & \text { SSCL } & 1.2 \mathrm{x} & 95 & 1\end{array}$

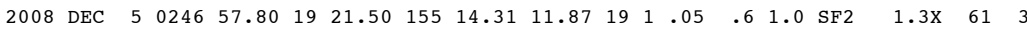

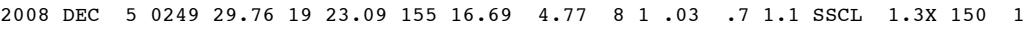

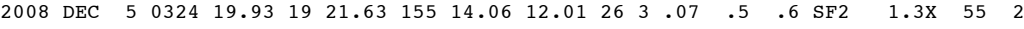
$\begin{array}{lllllllllllllllllll}2008 & \mathrm{DEC} & 5 & 0337 & 38.50 & 19 & 20.33 & 155 & 6.53 & 6.70 & 21 & 1 & .11 & .6 & 1.1 & \mathrm{SF} 4 & 1.4 \mathrm{X} & 148 & 6\end{array}$

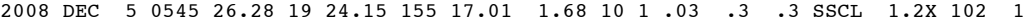

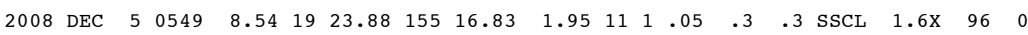

$\begin{array}{lllllllllllllllllll}2008 & \text { DEC } & 5 & 0553 & 14.98 & 19 & 24.18 & 155 & 16.75 & 1.81 & 23 & 5 & .09 & .3 & .2 & \text { SSCL } & 2.2 \mathrm{x} & 110 & 1\end{array}$

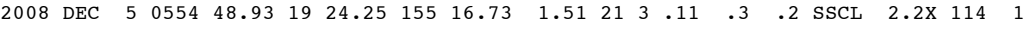
$\begin{array}{lllllllllllllllllll}2008 & \text { DEC } & 5 & 0557 & 20.54 & 19 & 24.59 & 155 & 16.34 & 4.82 & 14 & 1 & .07 & .6 & .7 & \text { SNCL } & 1.9 \times & 138 & 1\end{array}$ 2008 DEC $5 \begin{array}{lllllllllllllllll}0602 & 17.65 & 19 & 23.88 & 155 & 16.95 & 4.17 & 11 & 1 & .05 & .6 & .6 & \text { SSCL } & 1.6 \mathrm{X} & 94 & 1\end{array}$

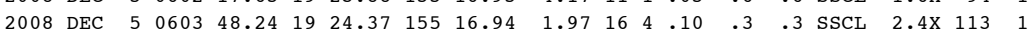

$\begin{array}{lllllllllllllllllll}2008 & \text { DEC } & 5 & 0604 & 56.58 & 19 & 24.10 & 155 & 17.09 & 1.76 & 11 & 1 & .04 & .3 & .3 & \text { SSCL } & 1.9 \mathrm{X} & 97 & 1\end{array}$

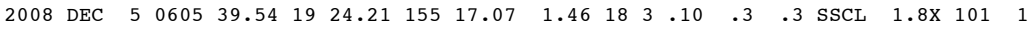

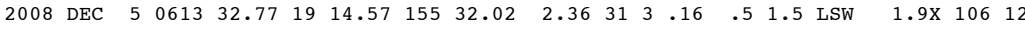

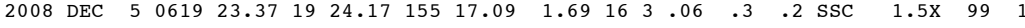

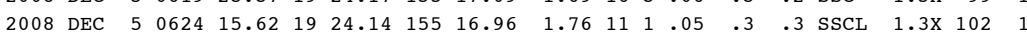

--ORIGIN TIME (HST)-- -LAT N-- --LON W-- DEPTH N N RMS ERH ERZ LOC PREF AZ MIN YEAR MON DA HRMN SEC DEG MIN DEG MIN KM RD S SEC KM KM REMKS MAG GAP DS

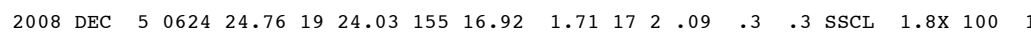

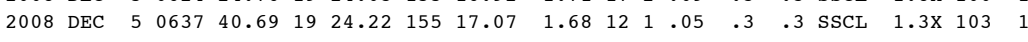

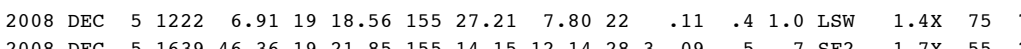

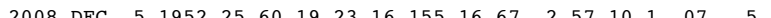

$\begin{array}{llllllllllllllllll}2008 & \text { DEC } & 5 & 2101 & 9.98 & 19 & 24.00 & 155 & 17.03 & 1.70 & 8 & 1.02 & .3 & .4 & \text { SSCL } & 1.3 \mathrm{X} & 96 & 1\end{array}$

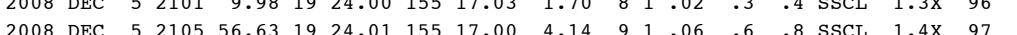

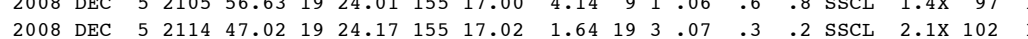
$\begin{array}{lllllllllllllll}2008 & \text { DEC } & 5 & 2118 & 32.00 & 19 & 24.21 & 155 & 16.70 & 2.43 & 12 & 1 & .08 & .4 & .3 \\ 3\end{array}$

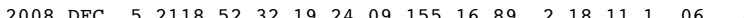

$\begin{array}{lllllllllllllllllll}2008 & \text { DEC } & 5 & 2123 & 56.68 & 19 & 23.91 & 155 & 16.64 & 3.41 & 10 & 1 & .05 & .5 & .5 & \text { SSCL } & 1.4 \times & 101 & 0\end{array}$

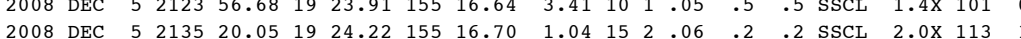

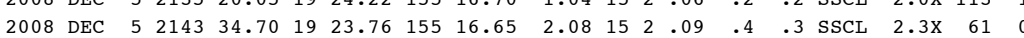

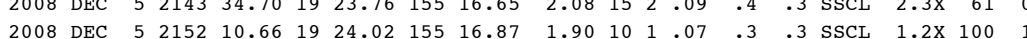

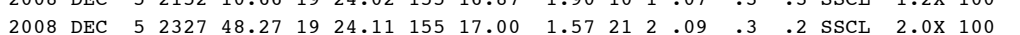

$\begin{array}{lllllllllllllllllll}2008 & \text { DEC } & 5 & 2345 & 36.88 & 19 & 23.92 & 155 & 16.88 & 2.00 & 13 & 3 & .06 & .3 & .3 & \text { SSCL } & 2.2 \mathrm{x} & 96 & 0\end{array}$

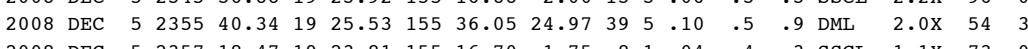

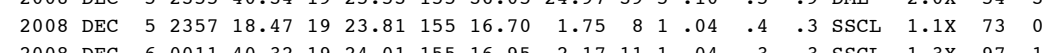

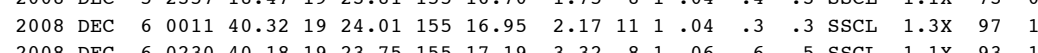

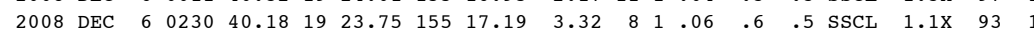

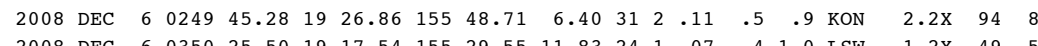

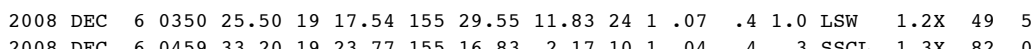
$\begin{array}{lllllllllllllllllll}2008 & \text { DEC } & 6 & 0459 & 33.20 & 19 & 23.77 & 155 & 16.83 & 2.17 & 10 & 1 & .04 & .4 & .3 & \text { SSCL } & 1.3 \mathrm{X} & 82 & 0\end{array}$

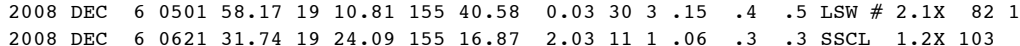

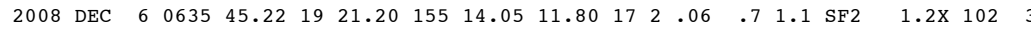

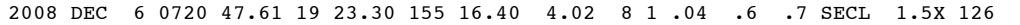

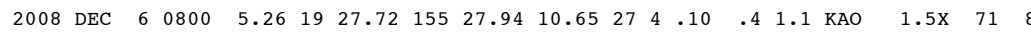

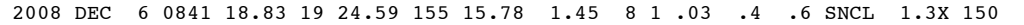

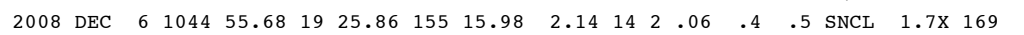

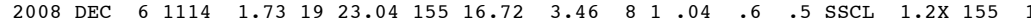

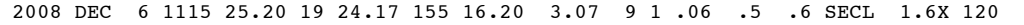

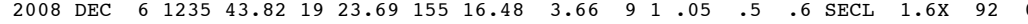

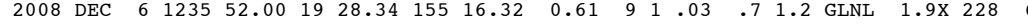

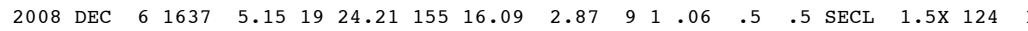

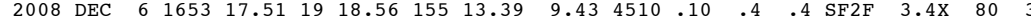

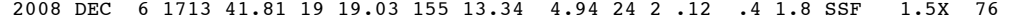

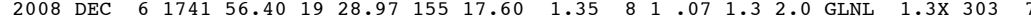
$\begin{array}{llllllllllllllllll}2008 & \text { DEC } & 6 & 1748 & 43.22 & 19 & 23.99 & 155 & 16.81 & 3.07 & 9 & 1 & .05 & .4 & .4 & \text { SSCL } & 1.4 \times & 101\end{array}$

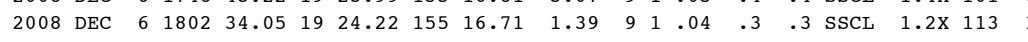

$\begin{array}{llllllllllllllllll}2008 & \text { DEC } & 6 & 1802 & 54.57 & 19 & 24.46 & 155 & 17.05 & 4.72 & 9 & 1 & .06 & .8 & 1.0 & \text { SSCL } & 1.2 \mathrm{X} & 113\end{array}$ $\begin{array}{lllllllllllllllllll}2008 & \text { DEC } & 6 & 1806 & 41.13 & 19 & 23.88 & 155 & 16.65 & 1.31 & 12 & 1 & .09 & .3 & .2 & \text { SSCL } & 1.6 x & 95 & 0\end{array}$

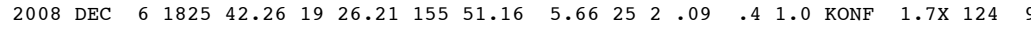

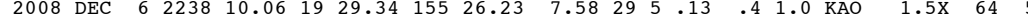

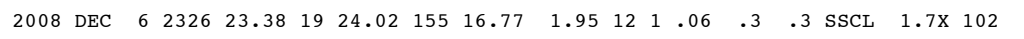


---ORIGIN TIME (HST)-- -LAT N-- --LON W-- DEPTH N N RMS ERH ERZ LOC PREF AZ MIN YEAR MON DA HRMN SEC DEG MIN DEG MIN KM RD S SEC KM KM REMKS MAG GAP DS $\begin{array}{lllllllllllllllllll}2008 & \text { DEC } & 6 & 2332 & 26.84 & 19 & 24.12 & 155 & 16.96 & 1.86 & 13 & 1 & .06 & .3 & .3 & \text { SSCL } & 1.6 \mathrm{X} & 102 & 1\end{array}$ $\begin{array}{lllllllllllllllllll}2008 & \text { DEC } & 6 & 2340 & 33.65 & 19 & 29.87 & 155 & 43.05 & 7.35 & 21 & 3 & .13 & .6 & 1.5 & \text { KON } & 1.4 \mathrm{X} & 138 & 5\end{array}$

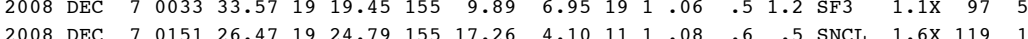

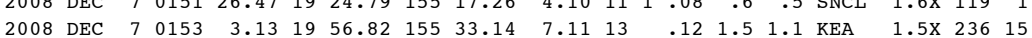

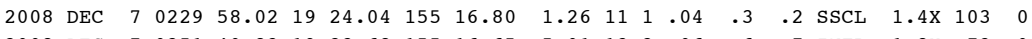
$\begin{array}{lllllllllllllllllll}2008 & \text { DEC } & 7 & 0251 & 40.83 & 19 & 23.68 & 155 & 16.65 & 5.01 & 13 & 2 & .06 & .6 & .7 & \text { INTL } & 1.8 \mathrm{X} & 73 & 0\end{array}$

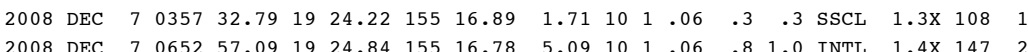

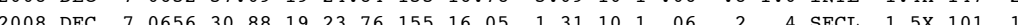

$\begin{array}{lllllllllllllllllll}2008 & \mathrm{DEC} & 7 & 0701 & 34.83 & 19 & 23.36 & 155 & 16.30 & 10.81 & 8 & 1 & .03 & 1.7 & 2.8 & \text { INTL } & 1.4 \mathrm{X} & 115 & 1\end{array}$ $\begin{array}{lllllllllllllllllll}2008 & \text { DEC } & 7 & 0955 & 44.70 & 19 & 24.09 & 155 & 17.02 & 1.50 & 8 & 1 & .05 & .3 & .3 & \text { SSCL } & 1.4 \mathrm{X} & 99 & 1\end{array}$ $\begin{array}{lllllllllllllllllll}2008 & \text { DEC } & 7 & 1026 & 33.33 & 19 & 24.41 & 155 & 16.71 & 4.37 & 10 & 1 & .02 & .6 & .7 & \text { SSCL } & 1.4 \mathrm{X} & 123 & 1 \\ 2008 & \mathrm{DEC} & 7 & 1032 & 9.47 & 19 & 24.36 & 155 & 16.81 & 1.42 & 8 & 1 & .06 & .3 & .4 & \text { SSCL } & 1.1 \mathrm{X} & 117 & 1\end{array}$

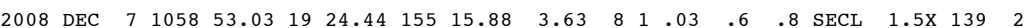

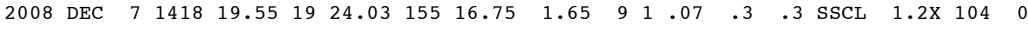

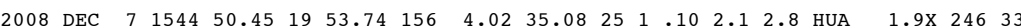

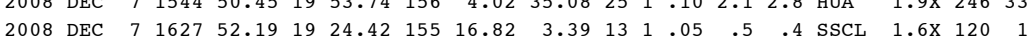

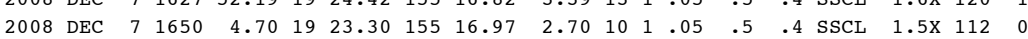

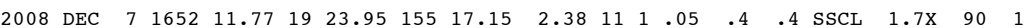

$\begin{array}{lllllllllllllllllll}2008 & \text { DEC } & 7 & 1658 & 24.40 & 19 & 24.01 & 155 & 17.18 & 3.05 & 12 & 1 & .08 & .5 & .4 & \text { SSCL } & 1.7 \mathrm{X} & 92 & 1\end{array}$

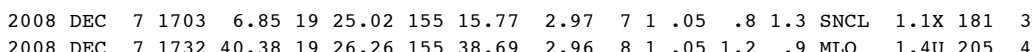

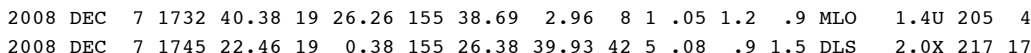

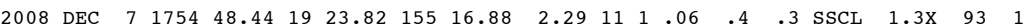

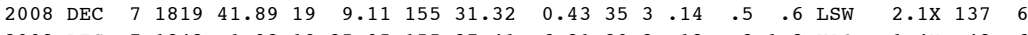

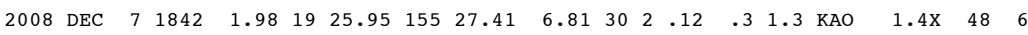

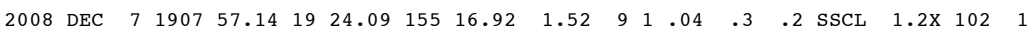

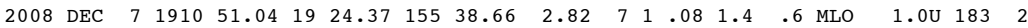

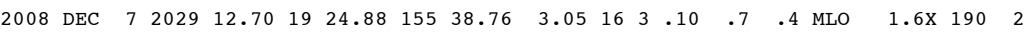

$\begin{array}{lllllllllllllllllll}2008 & \text { DEC } & 7 & 2102 & 22.15 & 19 & 26.10 & 155 & 39.69 & 6.44 & 13 & 1 & .08 & .6 & 1.4 & \text { MLO } & 1.2 \times & 123 & 5\end{array}$ 2008 DEC $7 \begin{array}{llllllllllllllllll}7 & 2136 & 58.27 & 19 & 11.53 & 155 & 24.45 & 36.37 & 36 & 1 & .10 & .8 & 1.5 & \text { DEP } & 1.6 \mathrm{X} & 162 & 5\end{array}$

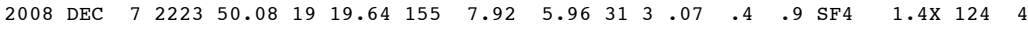

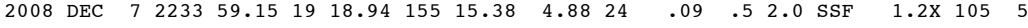

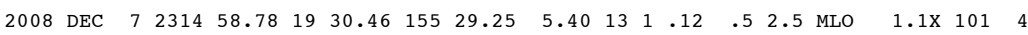

$\begin{array}{lllllllllllllllllll}2008 & \text { DEC } & 8 & 0000 & 10.09 & 19 & 24.66 & 155 & 38.70 & 2.89 & 8 & 1 & .07 & 1.1 & .5 & \text { MLO } & 1.0 \mathrm{U} & 187 & 2\end{array}$

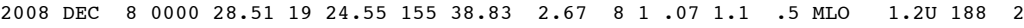

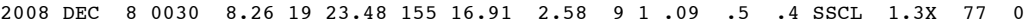
2008 DEC $8 \begin{array}{lllllllllllllllll}8 & 0049 & 48.99 & 19 & 24.09 & 155 & 16.91 & 2.48 & 11 & 1 & .05 & .4 & .3 & \text { SSCL } & 1.7 \times & 102 & 1\end{array}$

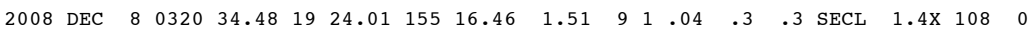

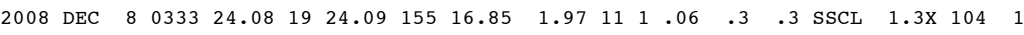

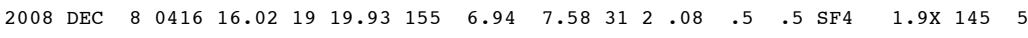

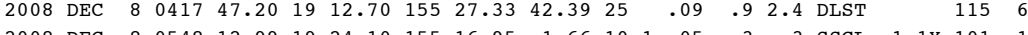

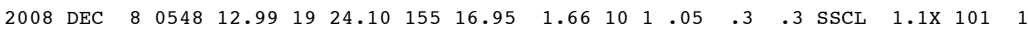

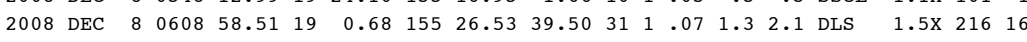

---ORIGIN TIME (HST)-- -LAT N-- --LON W-- DEPTH N N RMS ERH ERZ LOC PREF AZ MIN YEAR MON DA HRMN SEC DEG MIN DEG MIN KM RD S SEC KM KM REMKS MAG GAP DS

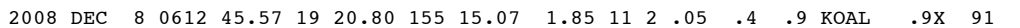

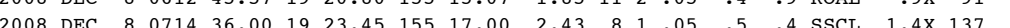

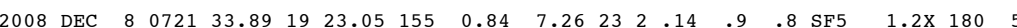

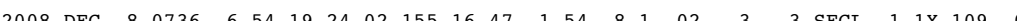

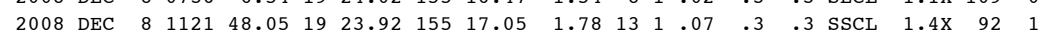

$\begin{array}{llllllllllllllllll}2008 & \text { DEC } & 8 & 1122 & 13.82 & 19 & 24.11 & 155 & 16.17 & 2.59 & 9 & 1 & .04 & .4 & .5 & \text { SECL } & 1.2 \times & 117\end{array}$

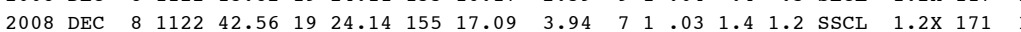
$\begin{array}{llllllllllllllllll}2008 & \text { DEC } & 8 & 1137 & 34.85 & 19 & 25.35 & 155 & 16.25 & 4.83 & 8 & 1 & .05 & 1.1 & 1.1 & \text { SNCL } & 1.6 \times & 197\end{array}$

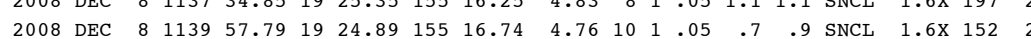
$\begin{array}{lllllllll} & \end{array}$

$\begin{array}{llllllllllllllllll}2008 & \text { DEC } & 8 & 1340 & 26.39 & 19 & 24.30 & 155 & 17.02 & 2.67 & 10 & 1 & .07 & .4 & .3 & \text { SSCL } & 1.4 \times & 107\end{array}$

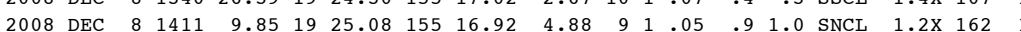

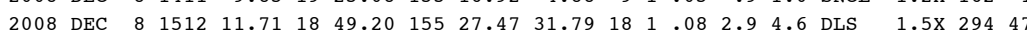

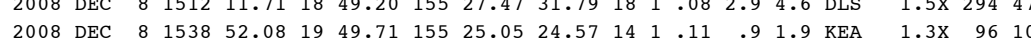

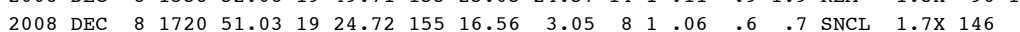

$\begin{array}{llllllllllllllllll}2008 & \text { DEC } & 8 & 1749 & 1.40 & 19 & 24.05 & 155 & 16.67 & 2.80 & 7 & 1.04 & .8 & .8 & \text { SSCL } & 1.5 \times & 126 & 0\end{array}$ $\begin{array}{lllllllllllllllllll}2008 & \text { DEC } & 8 & 1754 & 28.24 & 19 & 26.55 & 155 & 38.37 & 2.78 & 10 & 1 & .07 & 1.1 & .8 & \text { MLO } & 1.2 \mathrm{U} & 201 & 4\end{array}$

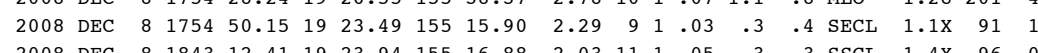
$\begin{array}{llllllllllllllllll}2008 & \text { DEC } & 8 & 1843 & 12.41 & 19 & 23.94 & 155 & 16.88 & 2.03 & 11 & 1.05 & .3 & .3 & \text { SSCL } & 1.4 \mathrm{x} & 96 & 0\end{array}$

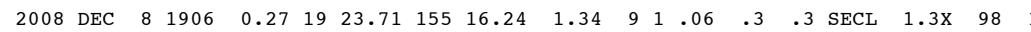

$\begin{array}{lllllllllllllllllll}2008 & \text { DEC } & 8 & 1926 & 19.39 & 19 & 25.62 & 155 & 16.05 & 2.93 & 11 & 1 & .03 & .7 & .6 & \text { SNCL } & 1.8 \times & 174 & 3\end{array}$

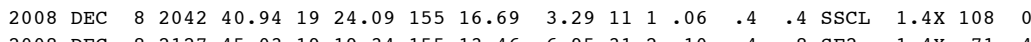

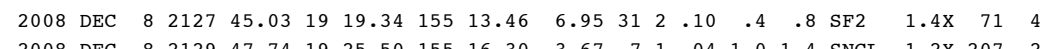

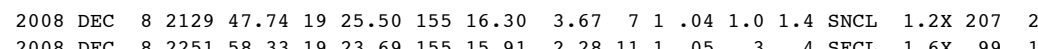
$\begin{array}{lllllllllllllllllll}2008 & \text { DEC } & 8 & 2251 & 58.33 & 19 & 23.69 & 155 & 15.91 & 2.28 & 11 & 1 & .05 & .3 & .4 & \text { SECL } & 1.6 \times & 99 & 1.64\end{array}$

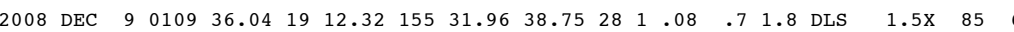

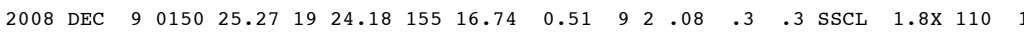

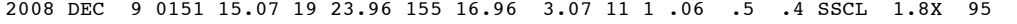

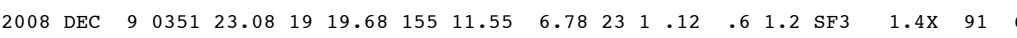

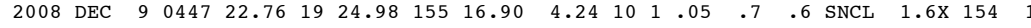

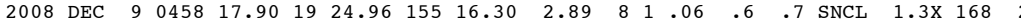

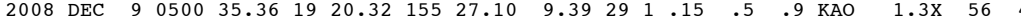

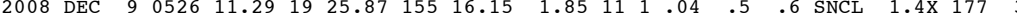
$\begin{array}{lllllllllllllllll}2008 & \text { DEC } & 9 & 0533 & 32.18 & 19 & 25.80 & 155 & 16.27 & 1.81 & 12 & 1 & .04 & .4 & .6 & \text { SNCL } & 1.5 \times\end{array}$

$\begin{array}{lllllllllllllllllll}2008 & \text { DEC } & 9 & 0543 & 11.27 & 19 & 25.63 & 155 & 16.24 & 2.06 & 25 & 4 & .12 & .3 & .4 & \text { SNC } & 2.2 \times & 104 & 2\end{array}$

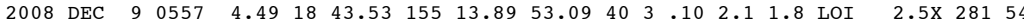

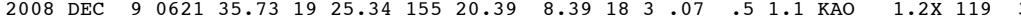

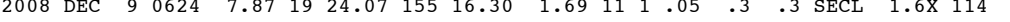

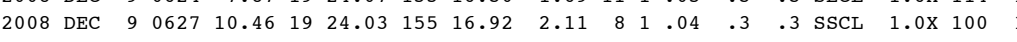

$\begin{array}{lllllllllllllllllll}2008 & \text { DEC } & 9 & 0910 & 42.39 & 19 & 24.14 & 155 & 16.78 & 3.24 & 8 & 1 & .06 & .6 & .6 & \text { SSCL } & 1.3 \times & 107 & 1\end{array}$ $\begin{array}{llllllllllllllllll}2008 & \text { DEC } & 9 & 1030 & 27.76 & 19 & 23.71 & 155 & 16.61 & 3.01 & 8 & 1.05 & .4 & .5 & \text { SSCL } & 1.1 \times & 72 & 0\end{array}$

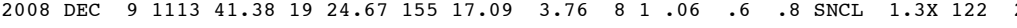

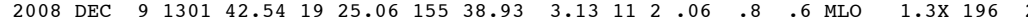

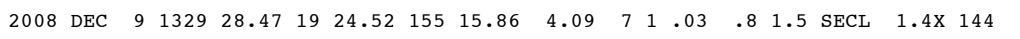


---ORIGIN TIME (HST)-- -LAT N-- --LON W-- DEPTH N N RMS ERH ERZ LOC PREF AZ MIN YEAR MON DA HRMN SEC DEG MIN DEG MIN KM RD S SEC KM KM REMKS MAG GAP DS $\begin{array}{lllllllllllllllllll}2008 & \text { DEC } & 9 & 1336 & 58.44 & 19 & 24.60 & 155 & 16.76 & 4.18 & 13 & 2 & .08 & .5 & .6 & \text { SNCL } & 1.9 \times & 131 & 1\end{array}$ $\begin{array}{lllllllllllllllllll}2008 & \text { DEC } & 9 & 1722 & 29.85 & 19 & 23.29 & 155 & 16.90 & 3.44 & 11 & 1 & .07 & .6 & .5 & \text { SSCL } & 1.3 \times & 111 & 0\end{array}$ 2008 DEC 92119 (1)

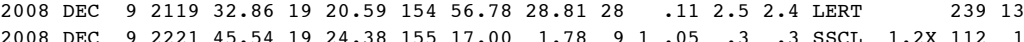

$\begin{array}{lllllllllllllllllll}2008 & \text { DEC } & 9 & 2221 & 55.19 & 19 & 23.61 & 155 & 16.56 & 1.43 & 9 & 1 & .04 & .3 & .2 & \text { SSCL } & 1.2 \mathrm{X} & 86 & 1\end{array}$ $\begin{array}{lllllllllllllllllll}2008 & \text { DEC } & 9 & 2237 & 29.19 & 19 & 24.39 & 155 & 16.97 & 2.50 & 10 & 1 & .05 & .4 & .4 & \text { SSCL } & 1.4 \times & 113 & 1\end{array}$

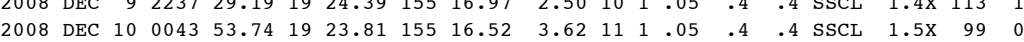

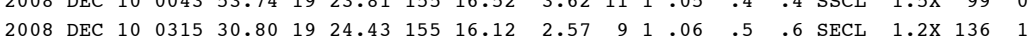

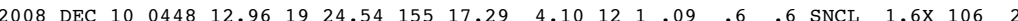

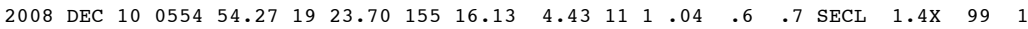

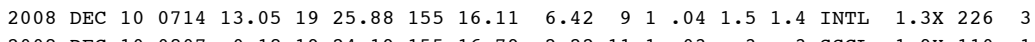

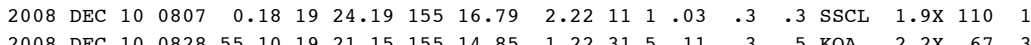

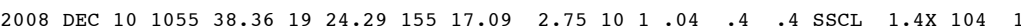

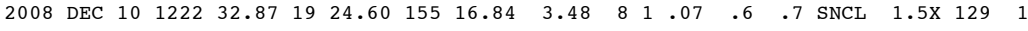

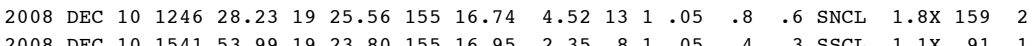

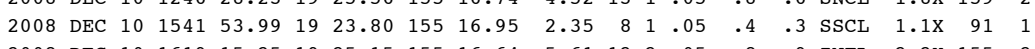

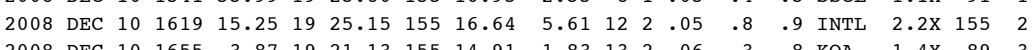

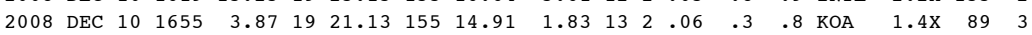

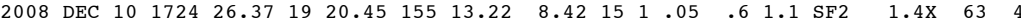

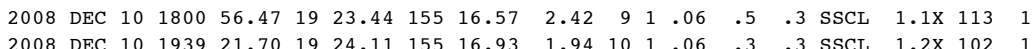

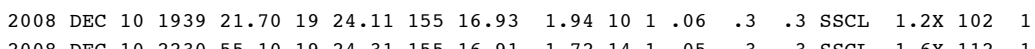

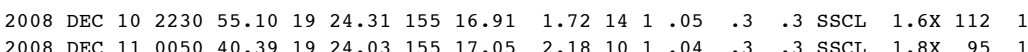
$\begin{array}{lllllllllllllllll}2008 & \text { DEC } 11 & 0050 & 40.39 & 19 & 24.03 & 155 & 17.05 & 2.18 & 10 & 1 & .04 & .3 & .3 & \text { SSCL } & 1.8 X & 95\end{array}$ $\begin{array}{lllllllllllllllllll}2008 & \text { DEC } & 11 & 0202 & 55.19 & 19 & 24.30 & 155 & 16.93 & 3.13 & 10 & 1 & .05 & .5 & .5 & \text { SSCL } & 1.4 \mathrm{X} & 111 & 1\end{array}$

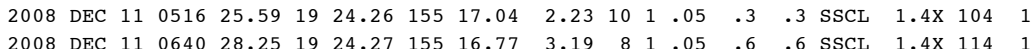

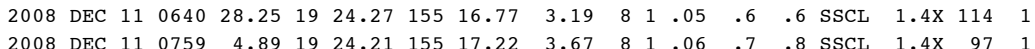

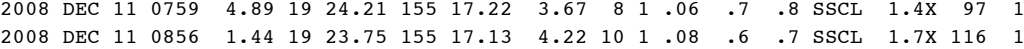

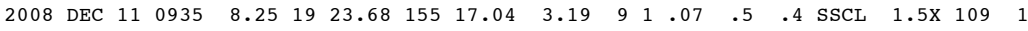

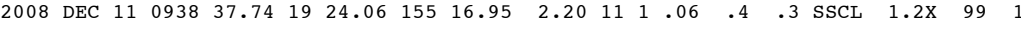

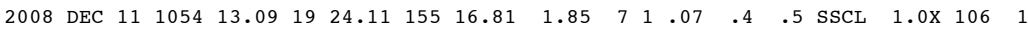

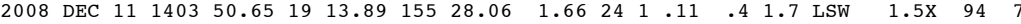

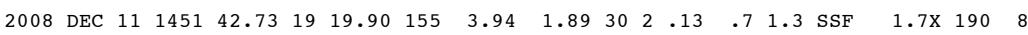

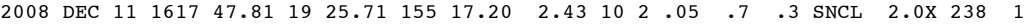

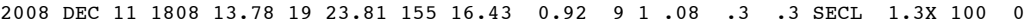
$\begin{array}{llllllllllllllllll}2008 & \text { DEC } 11 & 1813 & 45.53 & 19 & 25.02 & 155 & 16.67 & 7.97 & 7 & 1 & .05 & 1.3 & 2.2 & \text { INTL } & 1.4 X & 164 & 2\end{array}$ 2008 DEC $11 \quad 1839 \begin{array}{lllllllllllllll}57.87 & 19 & 24.35 & 155 & 16.99 & 3.37 & 15 & 2 & .04 & .3 & .3 & \text { SSCL } & 1.9 \times & 110 & 1\end{array}$

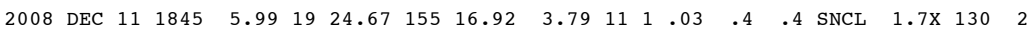

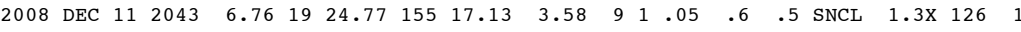

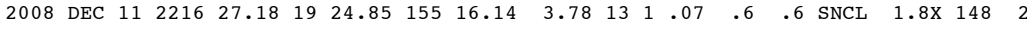

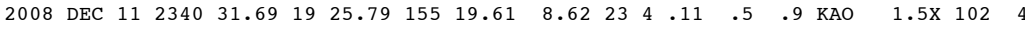

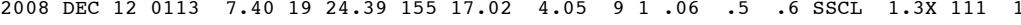

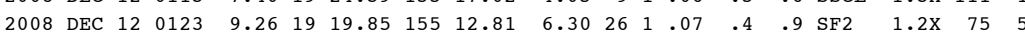

---ORTGIN TIME (HST)-- -LAT N-- --LON W-- DEPTH N N RMS ERH ERZ LOC PREF AZ MIN YEAR MON DA HRMN SEC DEG MIN DEG MIN KM RD S SEC KM KM REMKS MAG GAP DS

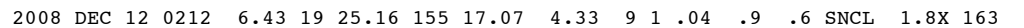

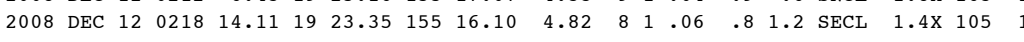

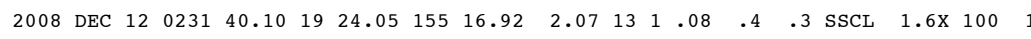

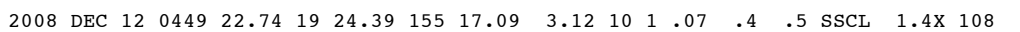

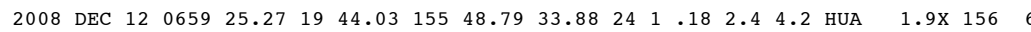

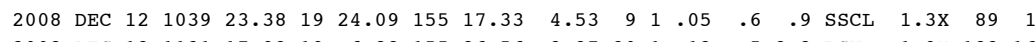

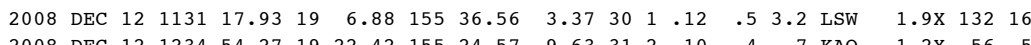

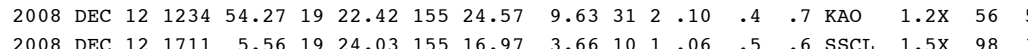

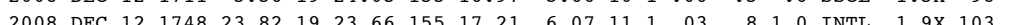

$\begin{array}{llllllllllllllllll}2008 & \text { DEC } 12 & 2002 & 43.45 & 19 & 24.78 & 155 & 16.37 & 3.36 & 9 & 1.06 & .6 & .7 & \text { SNCL } & 1.1 \times & 153 & 2\end{array}$

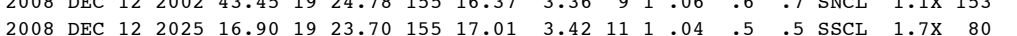

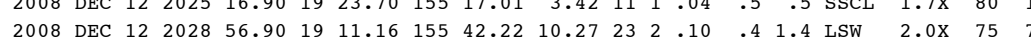

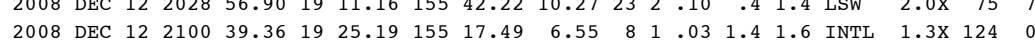

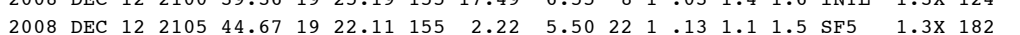

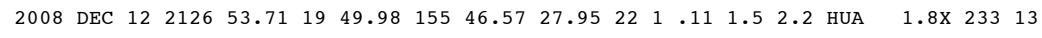

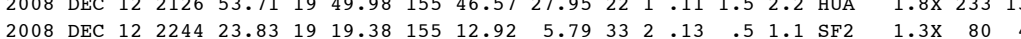

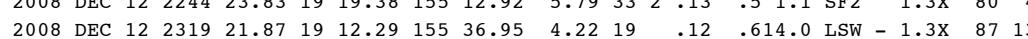

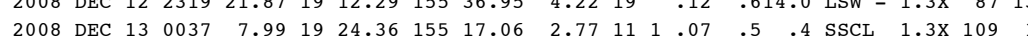

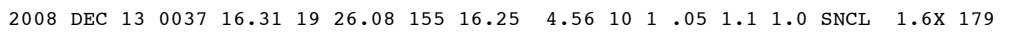

$\begin{array}{lllllllllllllllllll}2008 & \text { DEC } & 13 & 0111 & 10.65 & 19 & 24.38 & 155 & 16.86 & 3.23 & 11 & 1 & .05 & .5 & .4 & \text { SSCL } & 1.5 \times & 116 & 1\end{array}$

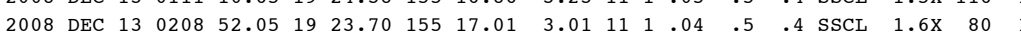

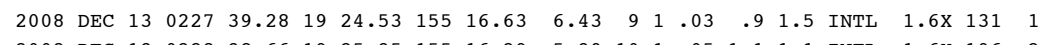

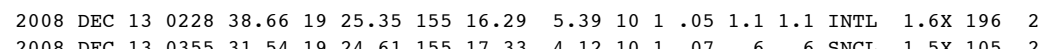

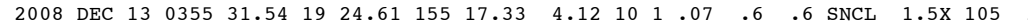

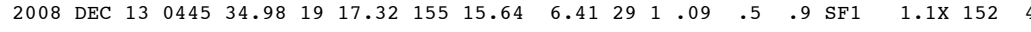

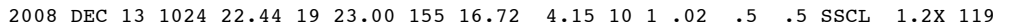

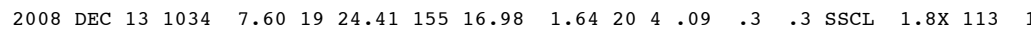
$\begin{array}{lllllllllllllllll}2008 & \text { DEC } 13 & 1038 & 4.23 & 19 & 19.41 & 155 & 8.75 & 6.07 & 25 & 2 & .11 & .5 & 1.4 & \text { SF4 } & 1.3 \times & 101\end{array}$

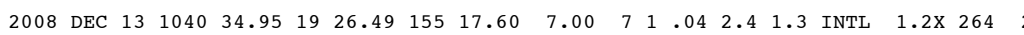

$\begin{array}{llllllllllllllllll}2008 & \text { DEC } & 13 & 1114 & 36.63 & 19 & 23.62 & 155 & 17.22 & 2.42 & 10 & 1 & .05 & .4 & .4 & \text { SSCL } & 1.4 \mathrm{X} & 106\end{array}$

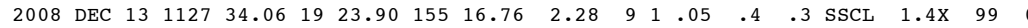

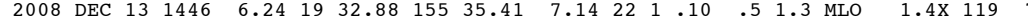

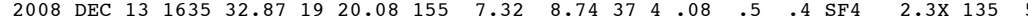

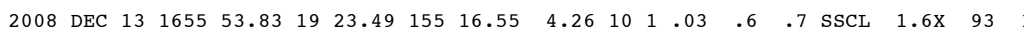

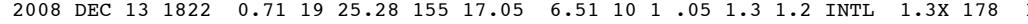
2008 DEC $131846 \begin{array}{llllllllllllll}17.56 & 19 & 24.35 & 155 & 17.21 & 2.49 & 10 & 1 & .06 & .4 & .4 & \text { SSCL } & 1.3 \times & 102\end{array}$

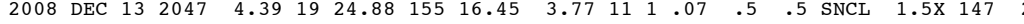

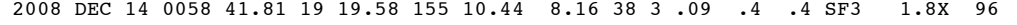

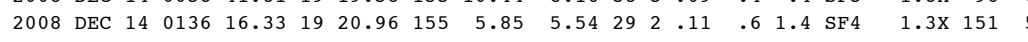

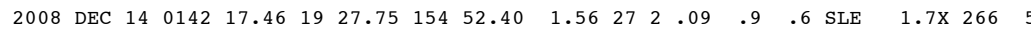

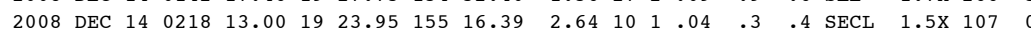

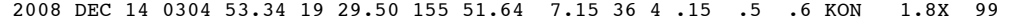

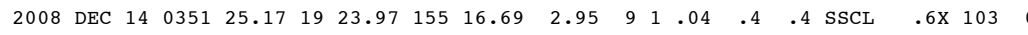

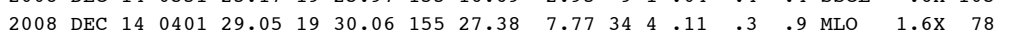


---ORIGIN TIME (HST)-- -LAT N-- --LON W-- DEPTH N N RMS ERH ERZ LOC PREF AZ MIN YEAR MON DA HRMN SEC DEG MIN DEG MIN KM RD S SEC KM KM REMKS MAG GAP DS $\begin{array}{lllllllllllllllllll}2008 & \text { DEC } 14 & 0503 & 12.41 & 19 & 25.11 & 155 & 16.66 & 5.90 & 13 & 2 & .06 & .8 & .9 & \text { INTL } & 1.8 \times & 154 & 2\end{array}$

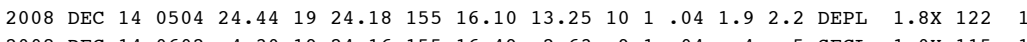

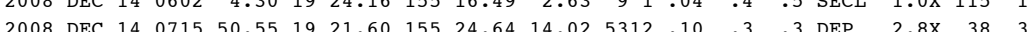

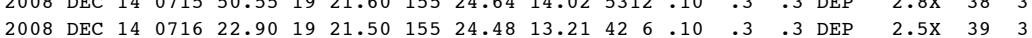

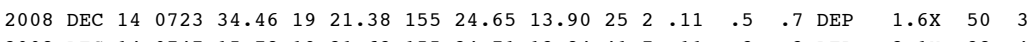

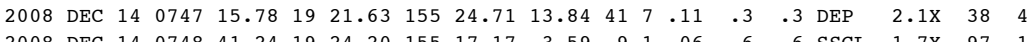

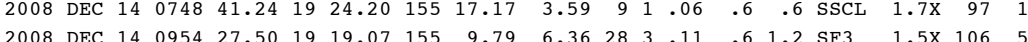

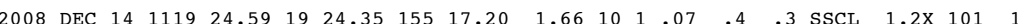

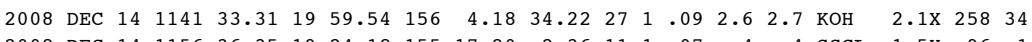

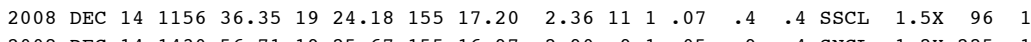

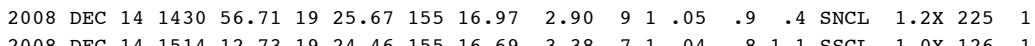

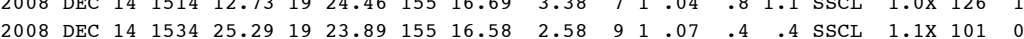

$\begin{array}{lllllllllllllllllll}2008 & \text { DEC } 14 & 1537 & 12.04 & 19 & 23.62 & 155 & 16.97 & 3.64 & 8 & 1 & .06 & .6 & .7 & \text { SSCL } & 1.2 \mathrm{X} & 98 & 0\end{array}$

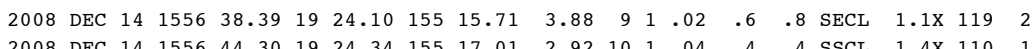

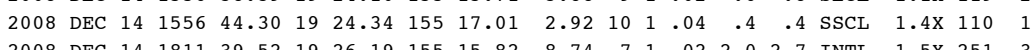

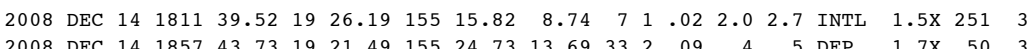

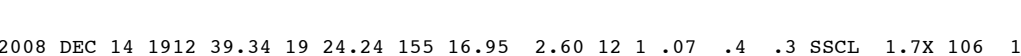

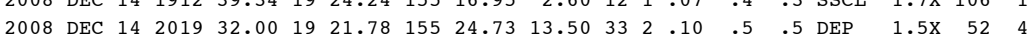

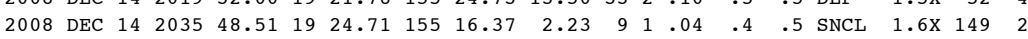

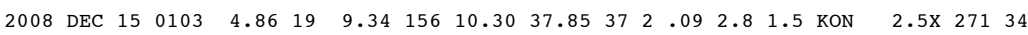

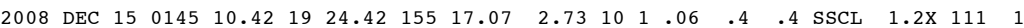

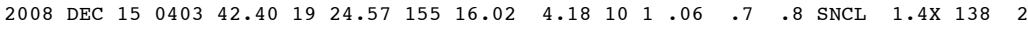

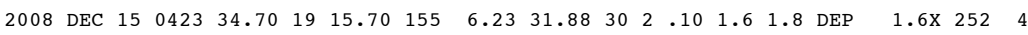

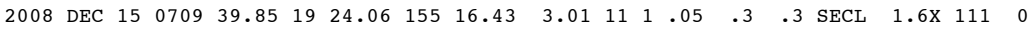

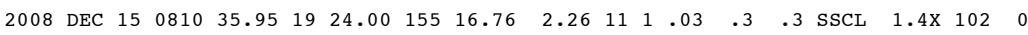

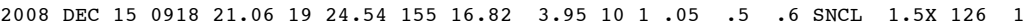

$\begin{array}{llllllllllllllllll}2008 & \text { DEC } 15 & 0946 & 58.51 & 20 & 7.89 & 155 & 46.74 & 22.24 & 23 & 1 & .11 & 3.1 & 2.6 & \text { KоH } & 2.0 \times & 288 & 0\end{array}$

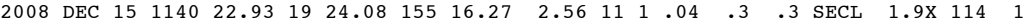

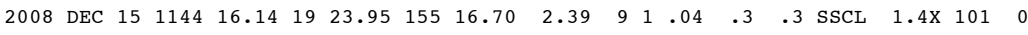

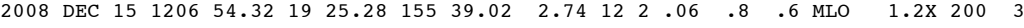

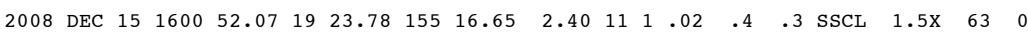

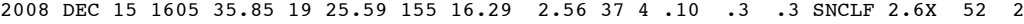

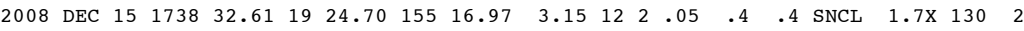

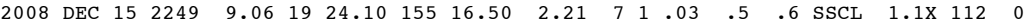

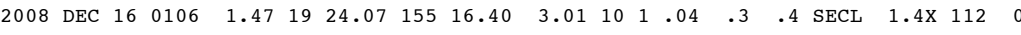

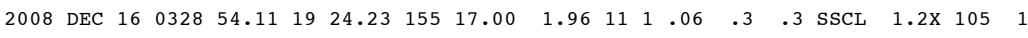

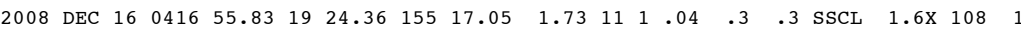

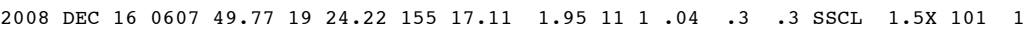

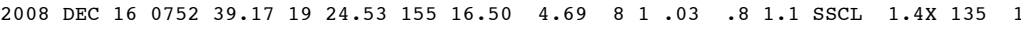

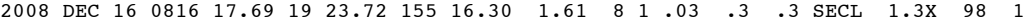

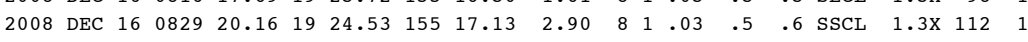

---ORTGIN TIME (HST)-- -LAT N-- --LON W-- DEPTH N N RMS ERH ERZ LOC PREF AZ MIN YEAR MON DA HRMN SEC DEG MIN DEG MIN KM RD S SEC KM KM REMRS MAG GAP DS $\begin{array}{lllllllllllllllll}2008 & \text { DEC } & 16 & 0841 & 15.07 & 19 & 24.82 & 155 & 16.55 & 3.83 & 12 & 1.06 & .5 & .4 & \text { SNCL } & 1.7 \times & 145\end{array}$

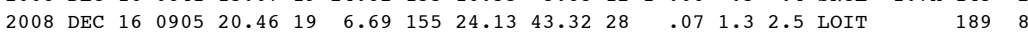

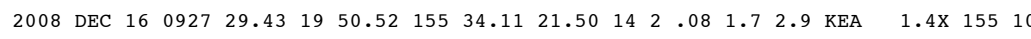

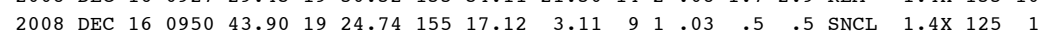

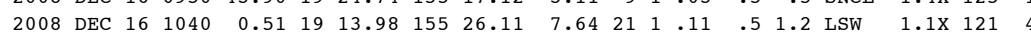

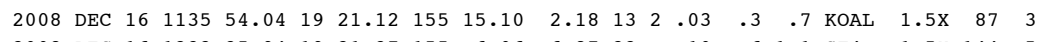

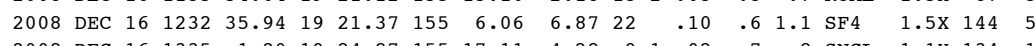

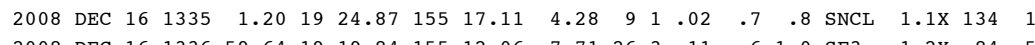

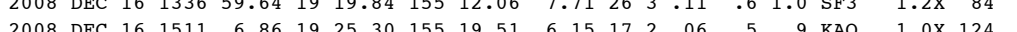

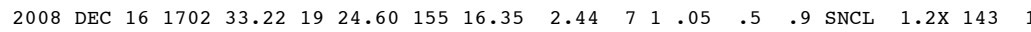

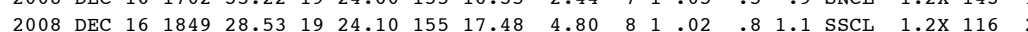

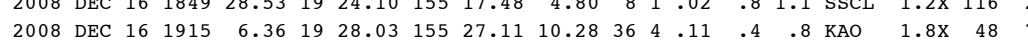

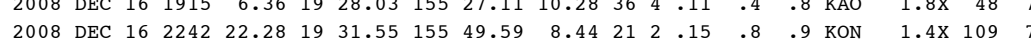

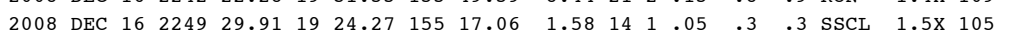

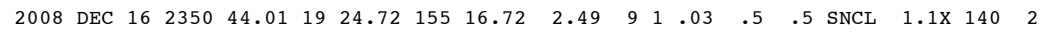

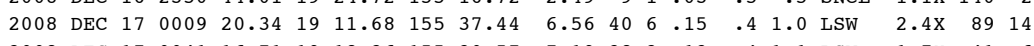

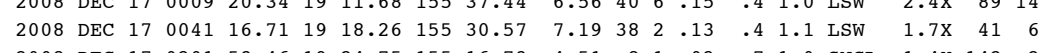

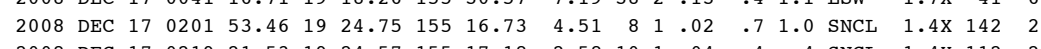

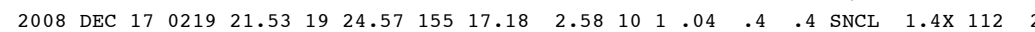

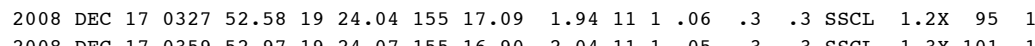

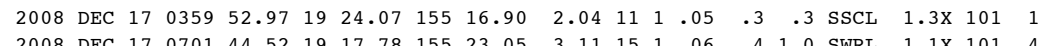

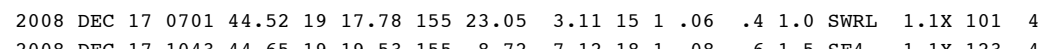

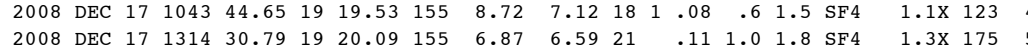

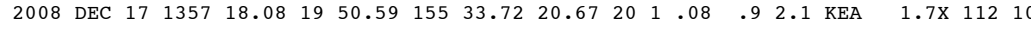

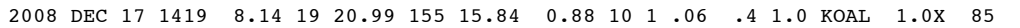

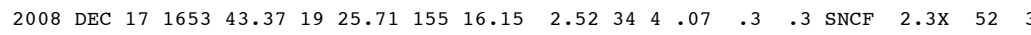

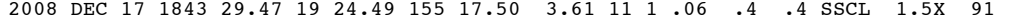

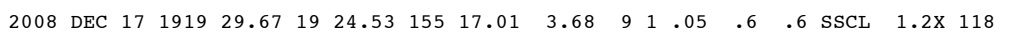

$\begin{array}{lllllllllllllllll}2008 & \text { DEC } 17 & 2002 & 38.28 & 19 & 24.27 & 155 & 17.21 & 3.20 & 8 & 1 & .04 & .6 & .6 & \text { SSCL } & 1.0 x & 99\end{array}$

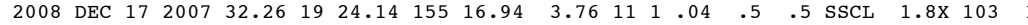

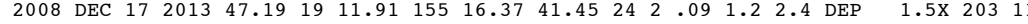
$\begin{array}{lllllllllllllllll}2008 & \text { DEC } 18 & 0153 & 34.85 & 19 & 24.53 & 155 & 17.24 & 2.89 & 10 & 1 & .06 & .4 & .4 & \text { SSCL } & 1.6 \times & 107\end{array}$

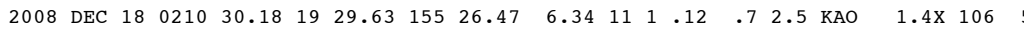

$\begin{array}{lllllllllllllllll}2008 & \text { DEC } 18 & 0550 & 18.87 & 19 & 24.64 & 155 & 16.79 & 3.92 & 11 & 1 & .05 & .5 & .5 & \text { SNCL } & 1.7 \times & 133\end{array}$

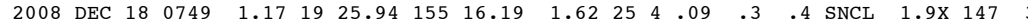

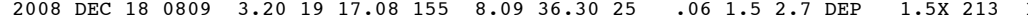

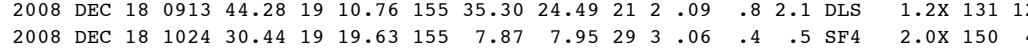

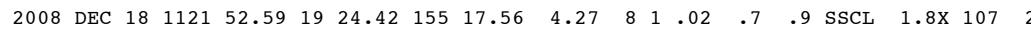

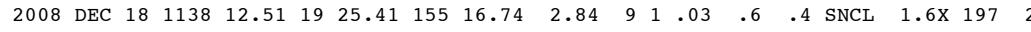

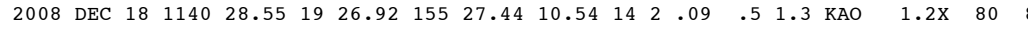

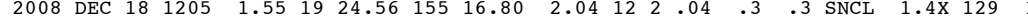

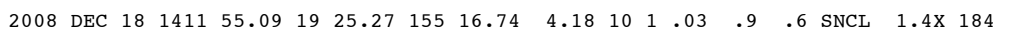


---ORIGIN TIME (HST)-- -LAT N-- --LON W-- DEPTH N N RMS ERH ERZ LOC PREF AZ MIN YEAR MON DA HRMN SEC DEG MIN DEG MIN KM RD S SEC KM KM REMKS MAG GAP DS $\begin{array}{llllllllllllllllll}2008 & \text { DEC } 18 & 1639 & 55.21 & 19 & 24.27 & 155 & 17.19 & 2.48 & 12 & 2 & .04 & .4 & .3 & \text { SSCL } & 1.6 \times & 100 & 1\end{array}$

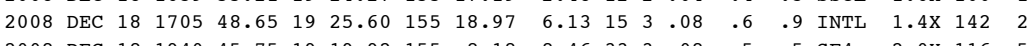

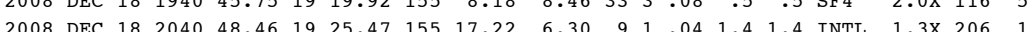

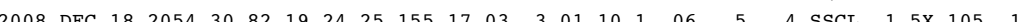

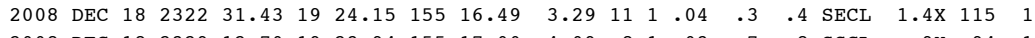

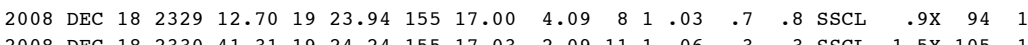

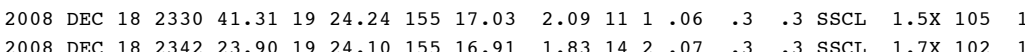

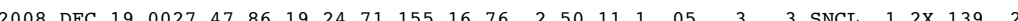

$\begin{array}{llllllllllllllllll}2008 & \text { DEC } 19 & 0027 & 50.00 & 19 & 16.34 & 155 & 23.28 & 2.39 & 20 & 1 & .08 & .4 & .9 & \text { SWR } & 1.4 \mathrm{X} & 115 & 4\end{array}$

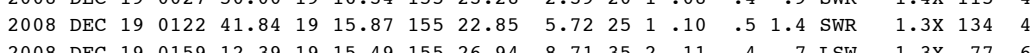

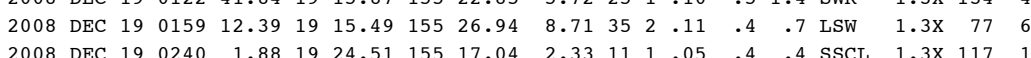

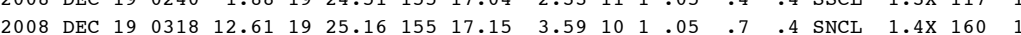

$\begin{array}{llllllllllllllllll}2008 & \text { DEC } 19 & 0330 & 45.98 & 19 & 18.30 & 155 & 13.35 & 6.18 & 30 & 3 & .08 & .5 & .8 & \text { SF2 } & 1.6 \mathrm{X} & 86 & 2\end{array}$

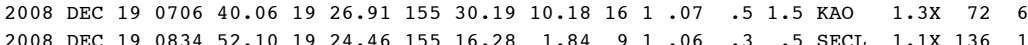

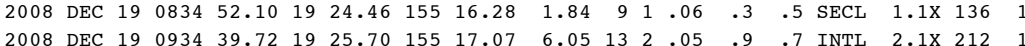

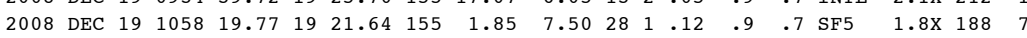

$\begin{array}{lllllllllllllllllll}2008 & \text { DEC } 19 & 1106 & 12.95 & 19 & 25.17 & 155 & 16.12 & 4.49 & 12 & 2 & .05 & .7 & .8 & \text { SNCL } & 1.8 \times & 158 & 2\end{array}$

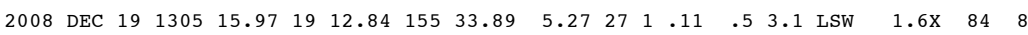

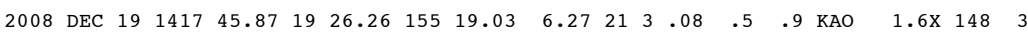

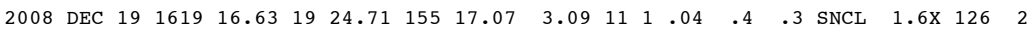

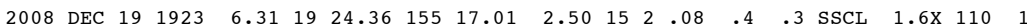

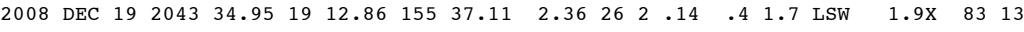

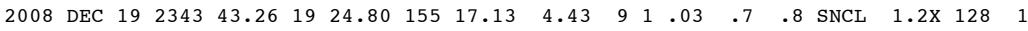

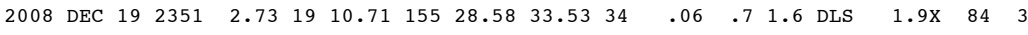
$\begin{array}{lllllllllllllllllll}2008 & \text { DEC } 20 & 0054 & 48.74 & 19 & 24.22 & 155 & 17.02 & 2.22 & 11 & 1 & .05 & .3 & .3 & \text { SSCL } & 1.7 \times & 104 & 1\end{array}$

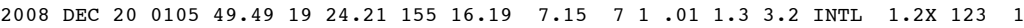

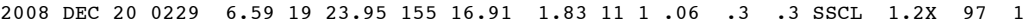

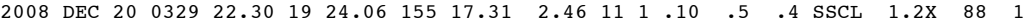
$\begin{array}{lllllllllllllllllll}2008 & \text { DEC } 20 & 0342 & 31.55 & 19 & 24.79 & 155 & 16.89 & 3.73 & 10 & 1 & .05 & .5 & .5 & \text { SNCL } & 1.1 \times & 139 & 2\end{array}$ $\begin{array}{llllllllllllllllll}2008 & \text { DEC } 20 & 0651 & 45.96 & 19 & 24.34 & 155 & 17.79 & 5.59 & 7 & 1 & .03 & 2.3 & 2.3 & \text { INTL } & .9 X & 229 & 2\end{array}$

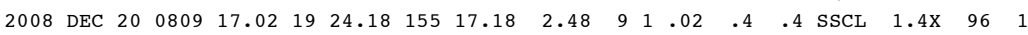

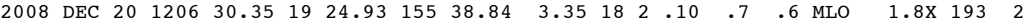

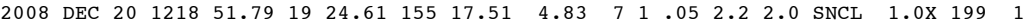

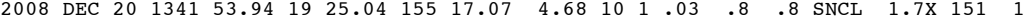

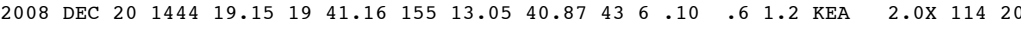

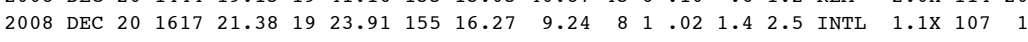

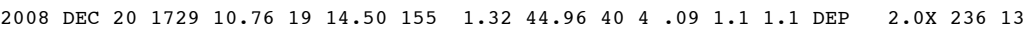

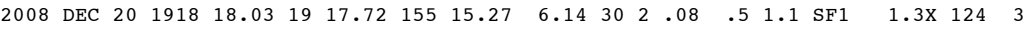

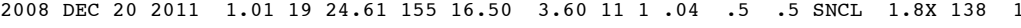

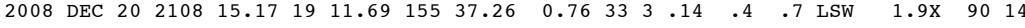

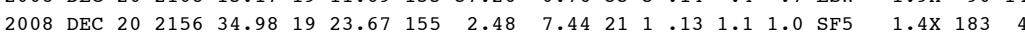

---ORIGIN TIME (HST)-- -LAT N-- --LON W-- DEPTH N N RMS ERH ERZ LOC PREF AZ MIN YEAR MON DA HRMN SEC DEG MIN DEG MIN KM RD S SEC KM KM REMKS MAG GAP DS

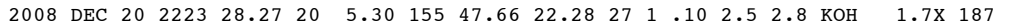
$\begin{array}{llllllllllllllll}2008 & \text { DEC } 20 & 2311 & 7.63 & 19 & 24.88 & 155 & 39.29 & 2.98 & 12 & .07 & 1.0 & .6 & \text { MLO } & 1.2 \times & 203\end{array}$

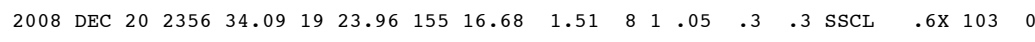
$\begin{array}{lllllllllllllllll}\text { DEC } 21 & 0949 & 40.65 & 19 & 23.92 & 155 & 16.83 & 2.00 & 11 & 1.10 & .4 & .3 & \text { SSCL } & 1.2 \mathrm{x} & 98\end{array}$

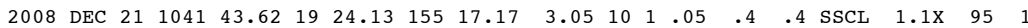

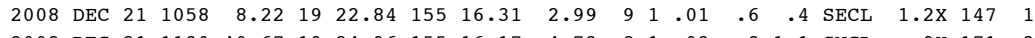

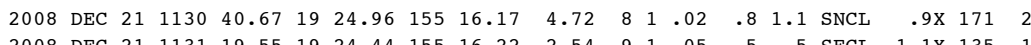

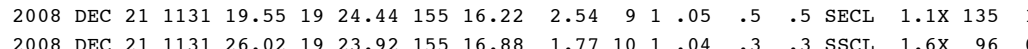
3 SSCL $1.6 \times \quad 96$

$\begin{array}{llllllllllllllllll}2008 & \text { DEC } & 21 & 1214 & 46.47 & 19 & 24.13 & 155 & 17.07 & 2.15 & 10 & 1.06 & .4 & .3 & \text { SSCL } & 1.2 \mathrm{x} & 99 & 1\end{array}$

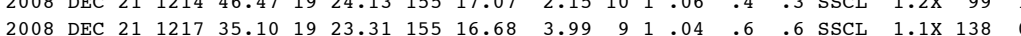

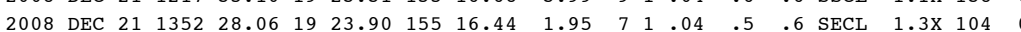

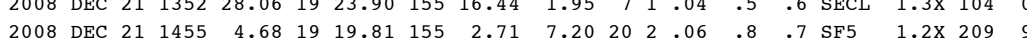

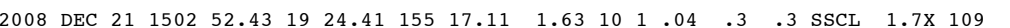

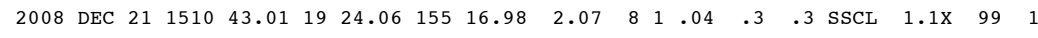
$\begin{array}{llllllllllllllllll}2008 & \text { DEC } 21 & 1511 & 31.75 & 19 & 24.11 & 155 & 17.03 & 2.08 & 15 & 2 & .06 & .3 & .3 & \text { SSCL } & 1.8 \mathrm{X} & 99 & 1\end{array}$

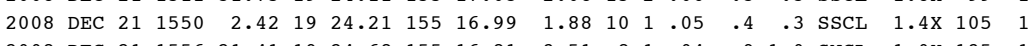

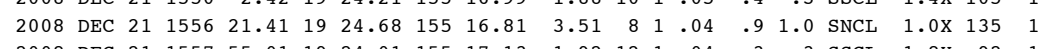

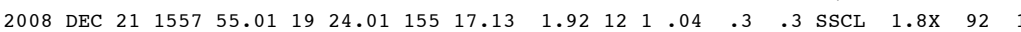

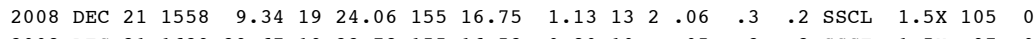
$\begin{array}{lllllllllllllllll}2008 & \text { DEC } 21 & 1629 & 39.67 & 19 & 23.78 & 155 & 16.52 & 0.80 & 10 & .05 & .3 & .2 & \text { SSCT } & 1.5 \mathrm{X} & 97 & 0\end{array}$

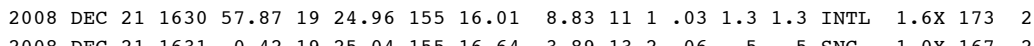

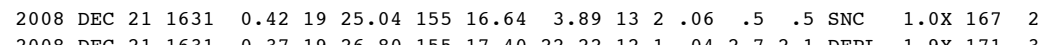

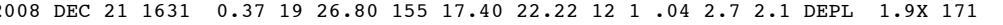

$\begin{array}{llllllllllllllllll}2008 & \text { DEC } & 21 & 1631 & 7.87 & 19 & 23.55 & 155 & 16.19 & 2.67 & 10 & 3 & .05 & .4 & .5 & \text { SECL } & 1.2 \mathrm{U} & 92\end{array}$

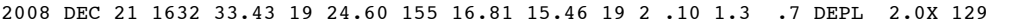
$\begin{array}{llllllllllllllllll}2008 & \text { DEC } 21 & 1633 & 37.81 & 19 & 25.03 & 155 & 17.74 & 11.19 & 16 & 2 & .05 & .7 & .7 & \text { INTL } & 1.8 X & 77 & 1\end{array}$

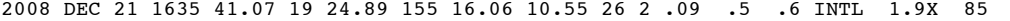

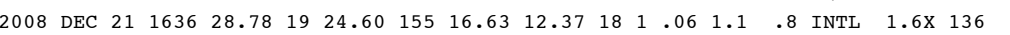

$\begin{array}{llllllllllllllllll}2008 & \text { DEC } 21 & 1637 & 7.77 & 19 & 25.03 & 155 & 17.10 & 10.65 & 21 & 2 & .10 & .7 & .7 & \text { INTL } & 1.5 \times & 138 & 1\end{array}$ $\begin{array}{lllllllllllllllll}2008 & \text { DEC } 21 & 1637 & 41.87 & 19 & 24.26 & 155 & 16.37 & 12.21 & 21 & 1 & .09 & .9 & .7 & \text { INTL } & 1.8 \times & 122\end{array}$

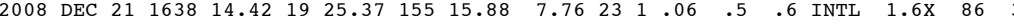

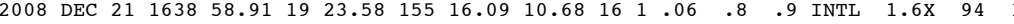

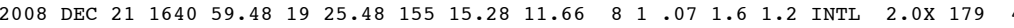

$\begin{array}{lllllllllllllllllll}2008 & \text { DEC } 21 & 1641 & 59.20 & 19 & 23.34 & 155 & 16.41 & 10.07 & 14 & 2 & .08 & .7 & .7 & \text { INTL } & 1.7 x & 112 & 2\end{array}$

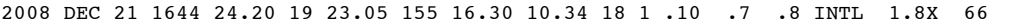

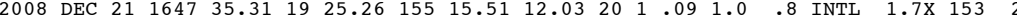

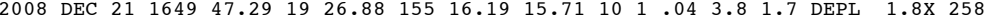

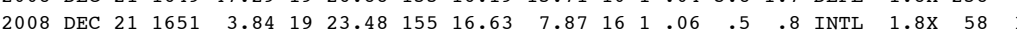

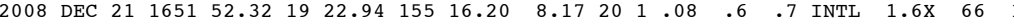
$\begin{array}{lllllllllllllllllll}2008 & \text { DEC } & 21 & 1652 & 34.57 & 19 & 23.72 & 155 & 16.67 & 6.23 & 13 & 1 & .08 & .8 & .9 & \text { INTL } & 1.5 \mathrm{X} & 67 & 0\end{array}$

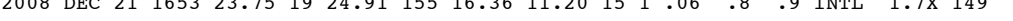

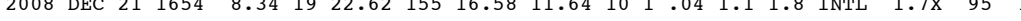

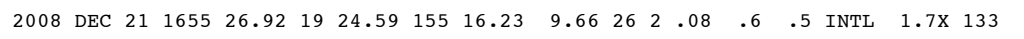


---ORIGIN TIME (HST)-- -LAT N-- --LON W-- DEPTH N N RMS ERH ERZ LOC PREF AZ MIN YEAR MON DA HRMN SEC DEG MIN DEG MIN KM RD S SEC KM KM REMKS MAG GAP DS $\begin{array}{llllllllllllllllll}2008 & \text { DEC } 21 & 1656 & 21.75 & 19 & 23.51 & 155 & 16.64 & 7.14 & 18 & 2 & .08 & .6 & .7 & \text { INTL } & 1.7 \mathrm{X} & 71 & 1\end{array}$

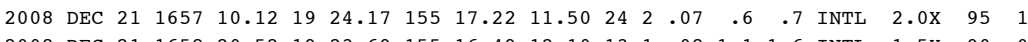

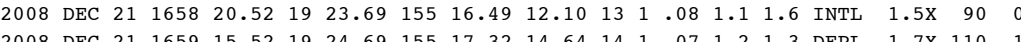

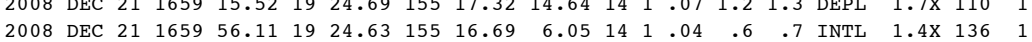

$\begin{array}{lllllllllllllllllll}2008 & \text { DEC } 21 & 1700 & 54.35 & 19 & 23.75 & 155 & 16.40 & 6.70 & 14 & 1 & .05 & .7 & .8 & \text { INTL } & 1.4 \mathrm{X} & 98 & 0\end{array}$

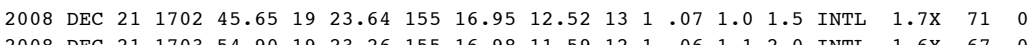

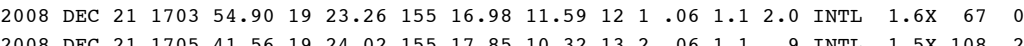

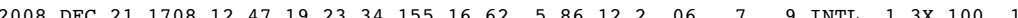

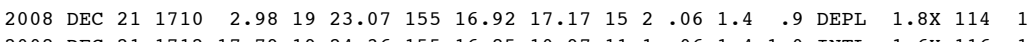

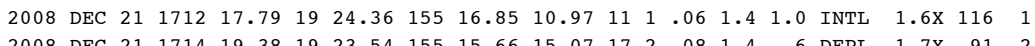

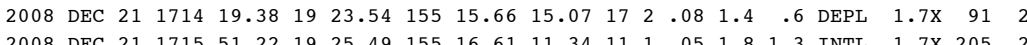

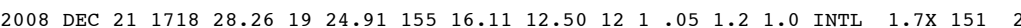

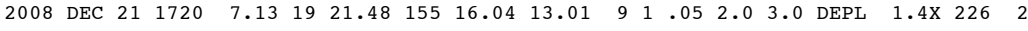

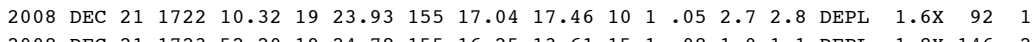

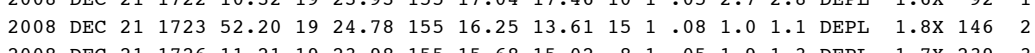

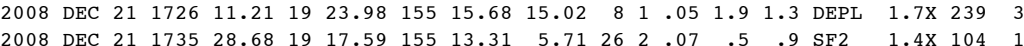

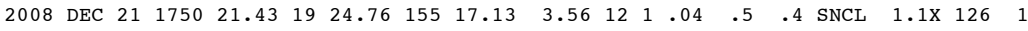

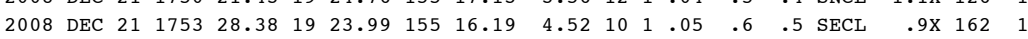

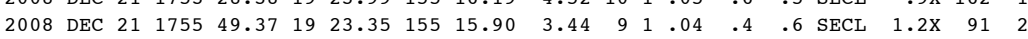

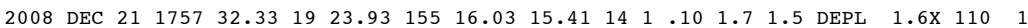

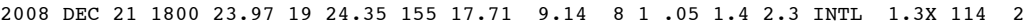

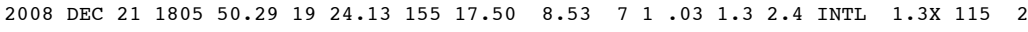

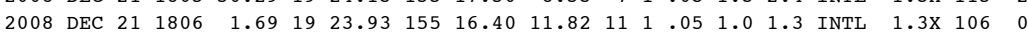

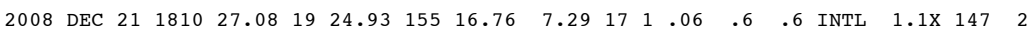

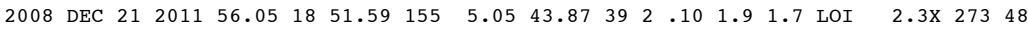

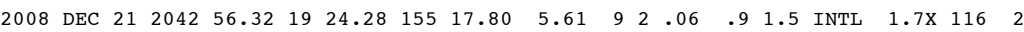

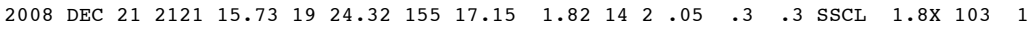

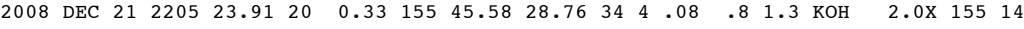

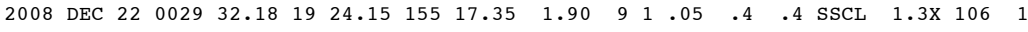

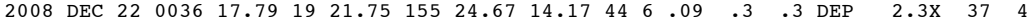

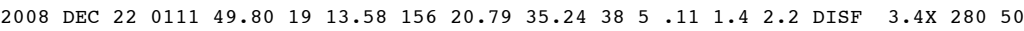

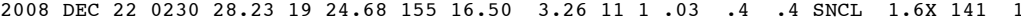
$\begin{array}{llllllllllllllllll}2008 & \text { DEC } 22 & 0336 & 35.55 & 19 & 24.86 & 155 & 16.61 & 2.40 & 11 & 1 & .05 & .4 & .4 & \text { SNCL } & 1.4 \mathrm{X} & 146 & 2\end{array}$

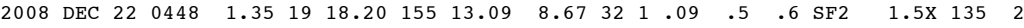

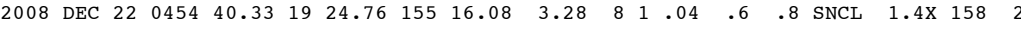

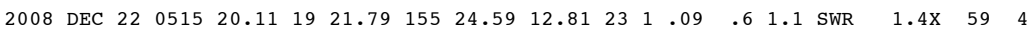

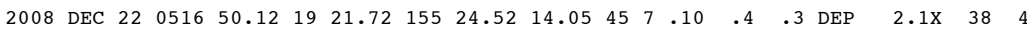

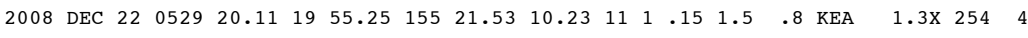

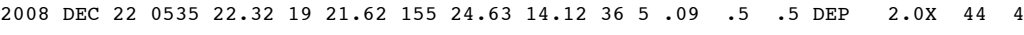

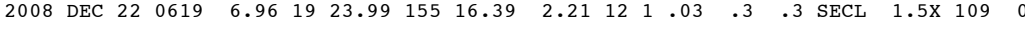

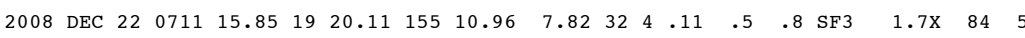

---ORTGIN TIME (HST)-- -LAT N-- --LON W-- DEPTH N N RMS ERH ERZ LOC PREF AZ MIN YEAR MON DA HRMN SEC DEG MIN DEG MIN KM RD S SEC KM KM REMKS MAG GAP DS

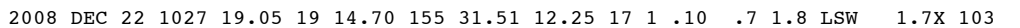

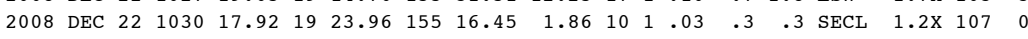

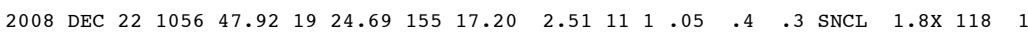

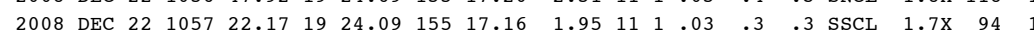

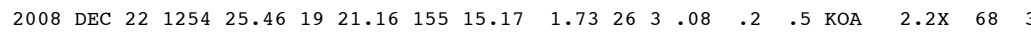

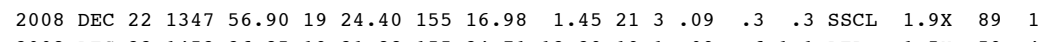
$\begin{array}{llllllllllllllllll}2008 & \text { DEC } 22 & 1453 & 36.85 & 19 & 21.88 & 155 & 24.71 & 13.39 & 19 & 1 & .09 & .6 & 1.1 & \text { DEP } & 1.5 \mathrm{X} & 59 & 4\end{array}$

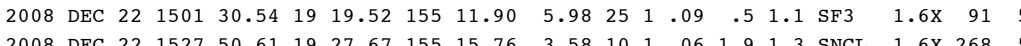

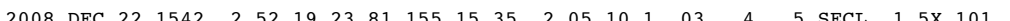

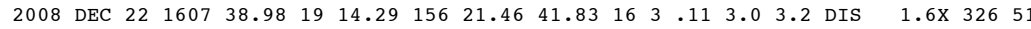

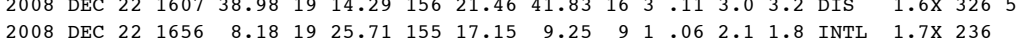

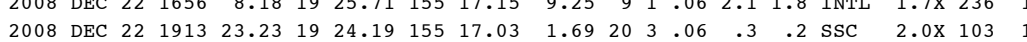

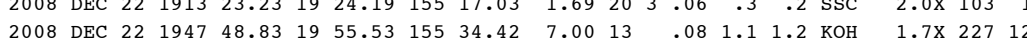

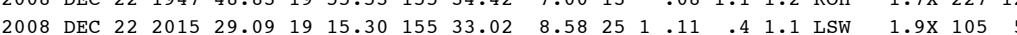

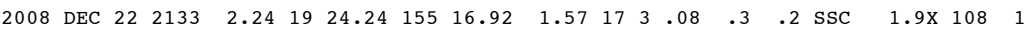

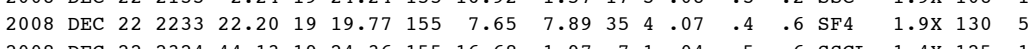

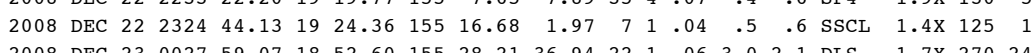

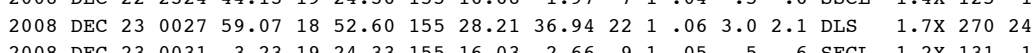

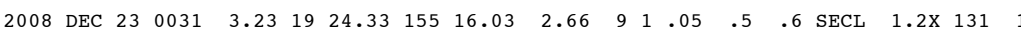

$\begin{array}{lllllllllllllllllll}2008 & \text { DEC } 23 & 0348 & 0.79 & 19 & 19.27 & 155 & 13.61 & 7.36 & 37 & 4 & .12 & .4 & .7 & \text { SF2 } & 1.8 \mathrm{X} & 67 & 4\end{array}$

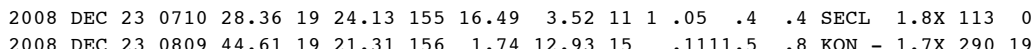

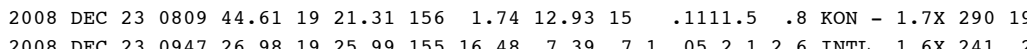

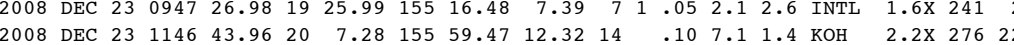

$\begin{array}{llllllllllllllllll}2008 & \text { DEC } 23 & 1214 & 59.81 & 19 & 24.07 & 155 & 16.90 & 2.37 & 11 & 1 & .03 & .4 & .3 & \text { SSCL } & 1.5 \times & 102 & 1\end{array}$

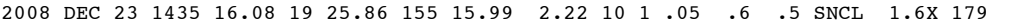

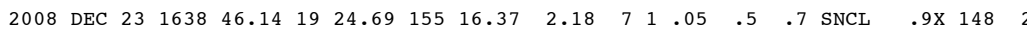

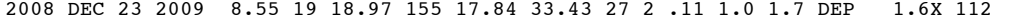

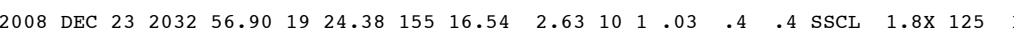

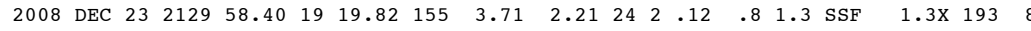

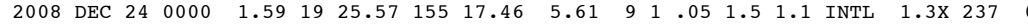

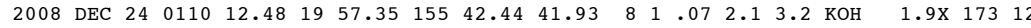

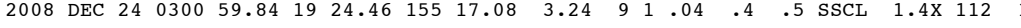

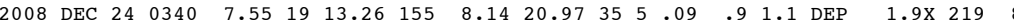

$\begin{array}{lllllllllllllllll}2008 & \text { DEC } 24 & 0352 & 53.61 & 19 & 24.23 & 155 & 17.03 & 3.87 & 9 & 1 & .03 & .5 & .6 & \text { SSCL } & 1.4 \mathrm{X} & 104\end{array}$

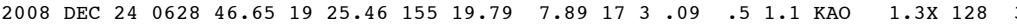

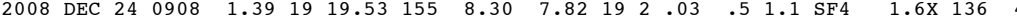

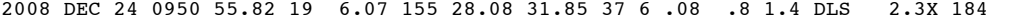

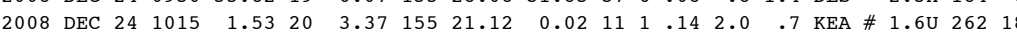

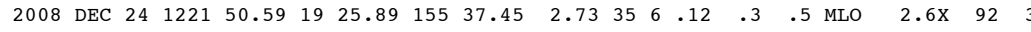
$\begin{array}{llllllllllllllllll}2008 & \text { DEC } 24 & 1229 & 27.44 & 20 & 1.05 & 155 & 37.05 & 13.02 & 6 & 1 & .07 & .8 & .8 & \text { кон } & 1.8 \mathrm{X} & 170 & 18\end{array}$

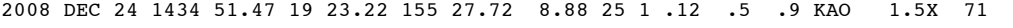

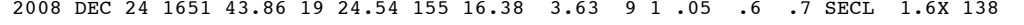

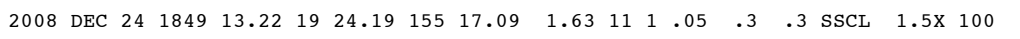


---ORIGIN TIME (HST)-- -LAT N-- --LON W-- DEPTH N N RMS ERH ERZ LOC PREF AZ MIN YEAR MON DA HRMN SEC DEG MIN DEG MIN KM RD S SEC KM KM REMKS MAG GAP DS

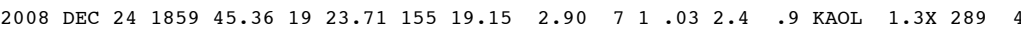

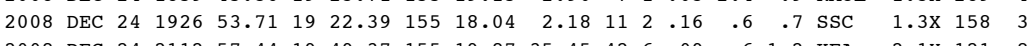

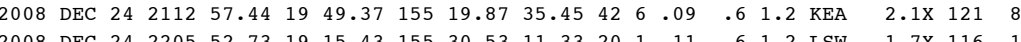

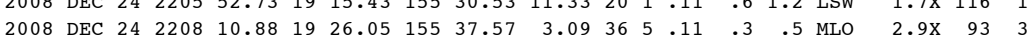

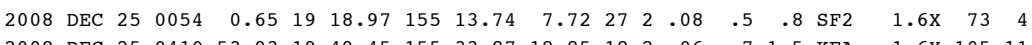

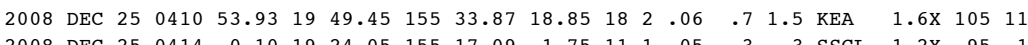

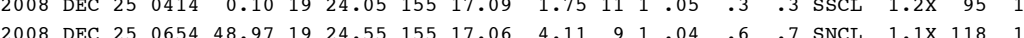

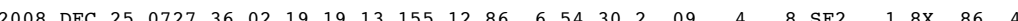

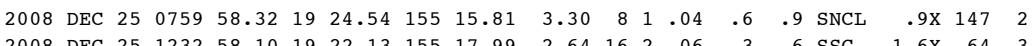

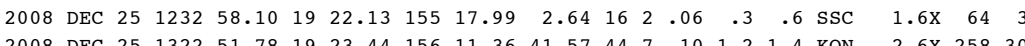

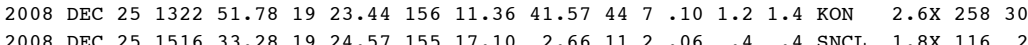

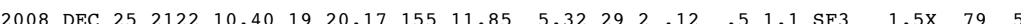

$\begin{array}{llllllllllllllllll}2008 & \text { DEC } 26 & 0115 & 30.89 & 19 & 23.81 & 155 & 17.26 & 3.28 & 11 & 2 & .04 & .6 & .4 & \text { SSCL } & 1.7 \mathrm{x} & 93 & 1\end{array}$

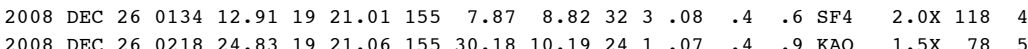

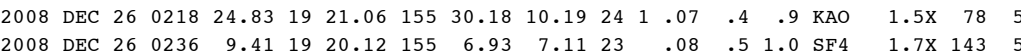

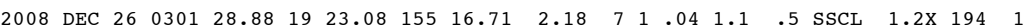

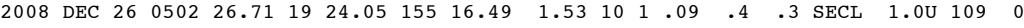

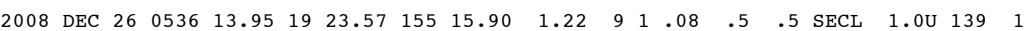

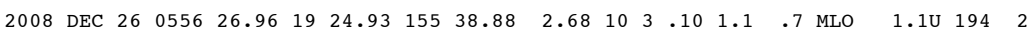

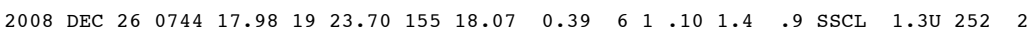

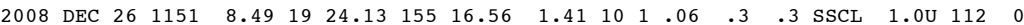

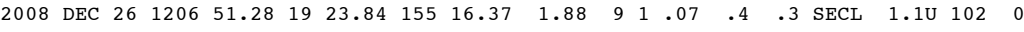

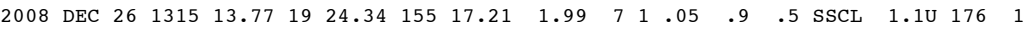

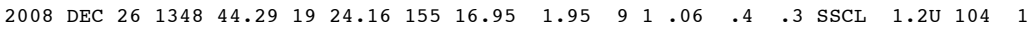

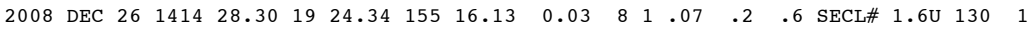

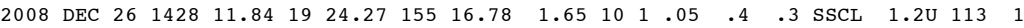

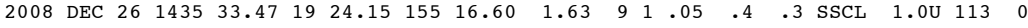

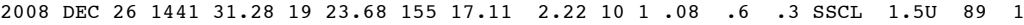

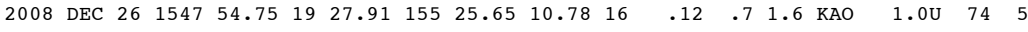

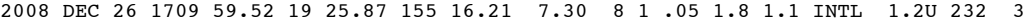

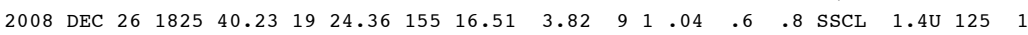

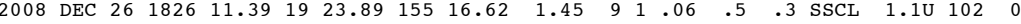

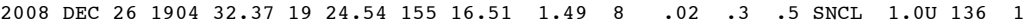

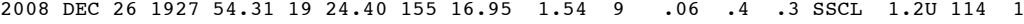

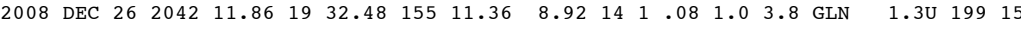

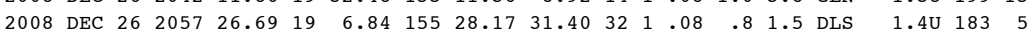

$\begin{array}{llllllllllllllllll}2008 & \text { DEC } 26 & 2235 & 48.25 & 19 & 24.15 & 155 & 16.82 & 1.74 & 10 & 1 & .07 & .4 & .3 & \text { SSCL } & 1.2 \mathrm{U} & 107 & 1\end{array}$

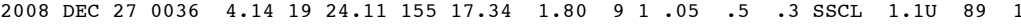

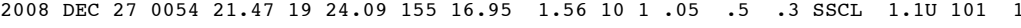

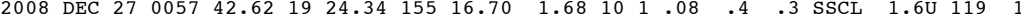

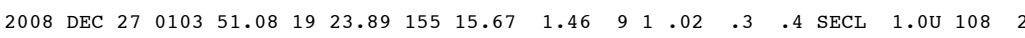

---ORIGIN TIME (HST)-- -LAT N-- --LON W-- DEPTH N N RMS ERH ERZ LOC PREF AZ MIN YEAR MON DA HRMN SEC DEG MIN DEG MIN KM RD S SEC KM KM REMKS MAG GAP DS

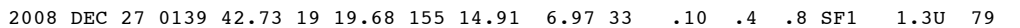

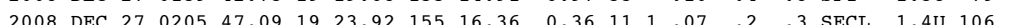

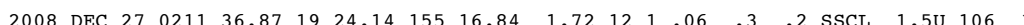
2008 DEC $27 \quad 0414 \quad 50.58 \quad 1926.94 \quad 15528.1010 .06 \quad 222010.4 \quad 1.0$ KAO 1.1067

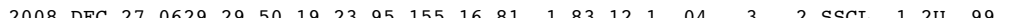

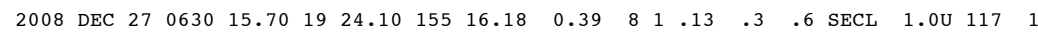

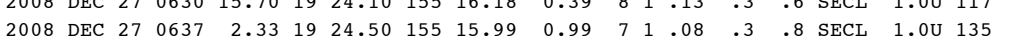

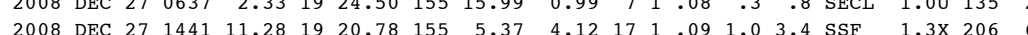

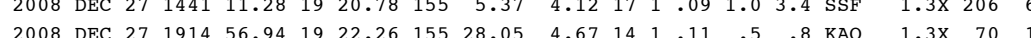

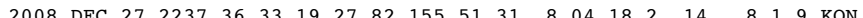

$\begin{array}{llllllllllllllllll}2008 & \text { DEC } 27 & 2300 & 8.27 & 19 & 25.39 & 155 & 26.99 & 7.33 & 16 & 1 & .12 & .5 & 1.6 & \text { KAO } & 1.3 \mathrm{X} & 65 & 5\end{array}$

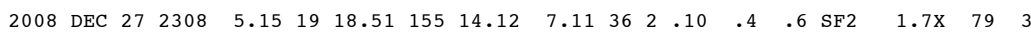

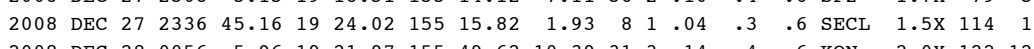

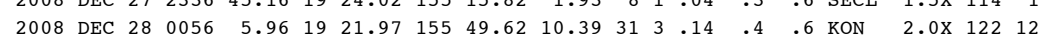

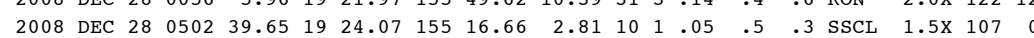

$\begin{array}{llllllllllllllllll}2008 & \text { DEC } 28 & 0529 & 1.56 & 19 & 24.38 & 155 & 15.92 & 2.81 & 10 & 1.05 & .4 & .5 & \text { SECL } & 1.7 \times & 130 & 1\end{array}$

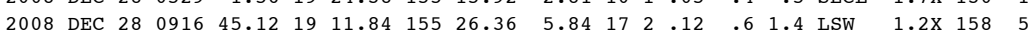

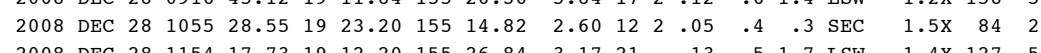

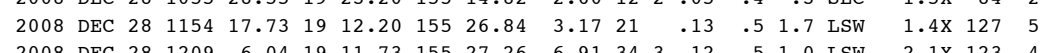

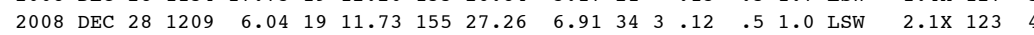

$\begin{array}{llllllllllllllllll}2008 & \text { DEC } 28 & 1219 & 19.91 & 19 & 19.53 & 155 & 4.48 & 5.40 & 20 & 1.12 & .8 & 2.2 & \text { SF5 } & 1.7 \times & 190 & 7\end{array}$

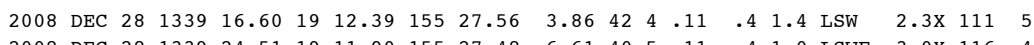

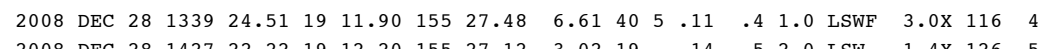

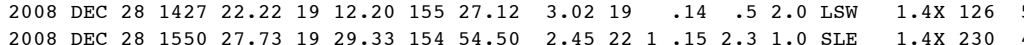

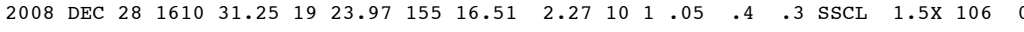

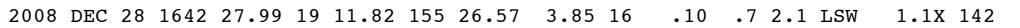

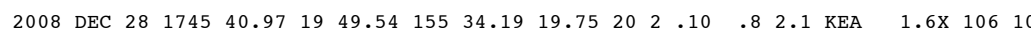

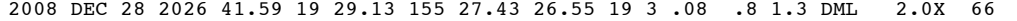

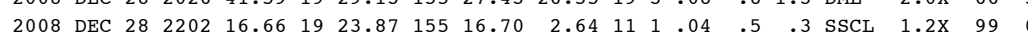

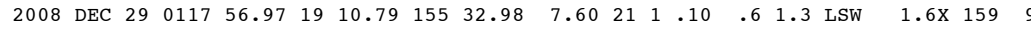

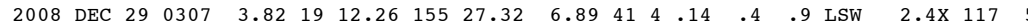

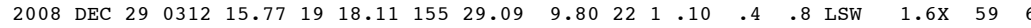

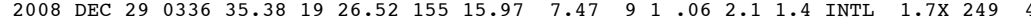

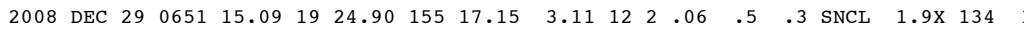

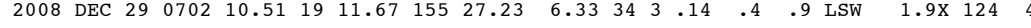

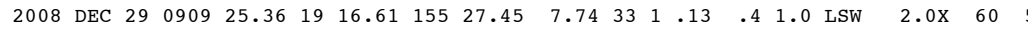

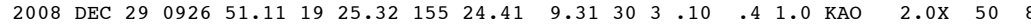

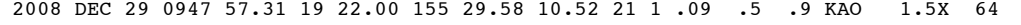

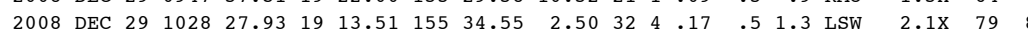

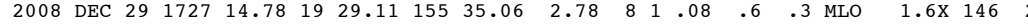

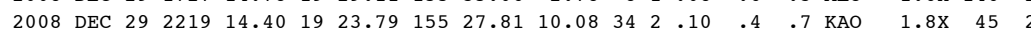

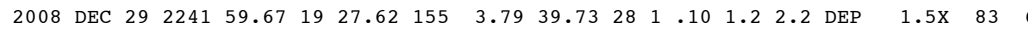

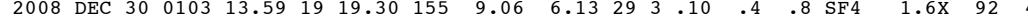

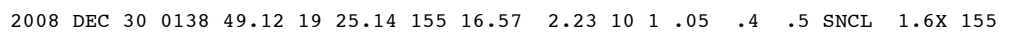


---ORIGIN TIME (HST)-- -LAT N-- --LON W-- DEPTH N N RMS ERH ERZ LOC PREF AZ MIN YEAR MON DA HRMN SEC DEG MIN DEG MIN KM RD S SEC KM KM REMKS MAG GAP DS

$\begin{array}{llllllllllllllllll}2008 & \text { DEC } 30 & 0156 & 19.62 & 19 & 24.91 & 155 & 29.69 & 8.67 & 27 & 2 & .09 & .3 & .9 & \text { KAO } & 1.3 \mathrm{X} & 65 & 6\end{array}$

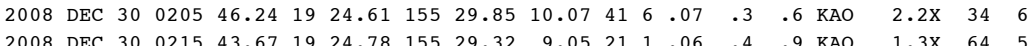

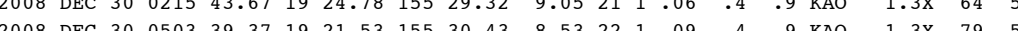

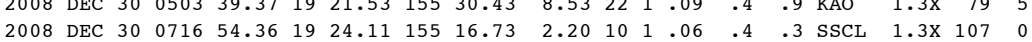

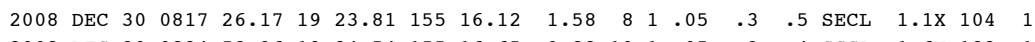

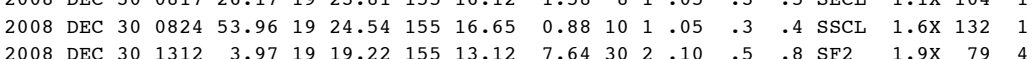

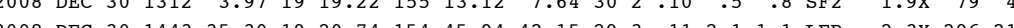

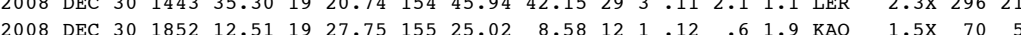

$\begin{array}{llllllllllllllllll}2008 & \text { DEC } 30 & 1855 & 53.12 & 19 & 31.38 & 155 & 42.74 & 8.78 & 20 & 3 & .11 & .8 & 1.7 & \text { MLO } & 1.3 \times & 174 & 6\end{array}$

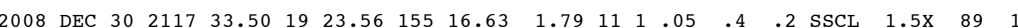
2008 DEC $30 \begin{array}{lllllllllllllllll}132 & 57.23 & 19 & 24.52 & 155 & 16.75 & 2.01 & 9 & 1 & .07 & .5 & .5 & \text { SSCL } & 1.4 \times & 127 & 1\end{array}$ 2008 DEC $30 \begin{array}{llllllllllllllll}2208 & 13.82 & 19 & 24.04 & 155 & 16.61 & 3.44 & 9 & 1 & .04 & .5 & .6 & \text { SSCL } & 1.4 \mathrm{X} & 107 & 0\end{array}$

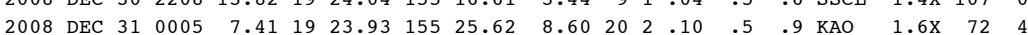

2008 DEC $31014935.281944 .76 \quad 156 \quad 7.59 \quad 16.64 \quad 19 \quad .11 \quad 3.015 .2$ HUA $-2.0 \times 24736$

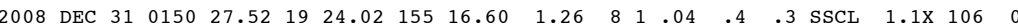

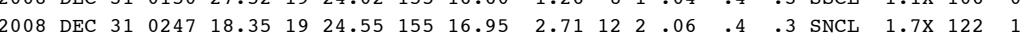

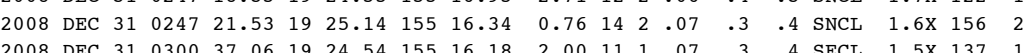

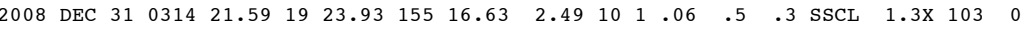

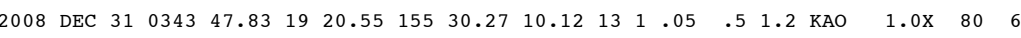

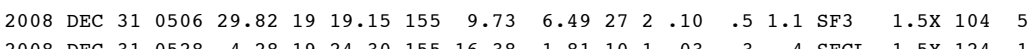

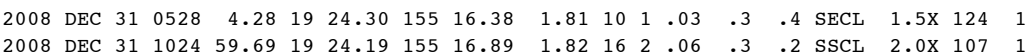

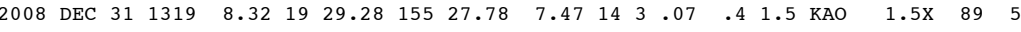

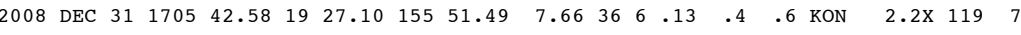

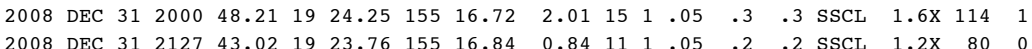


Table 5. List of the 51 events of magnitude 3.0 or greater, selected from table 4 .

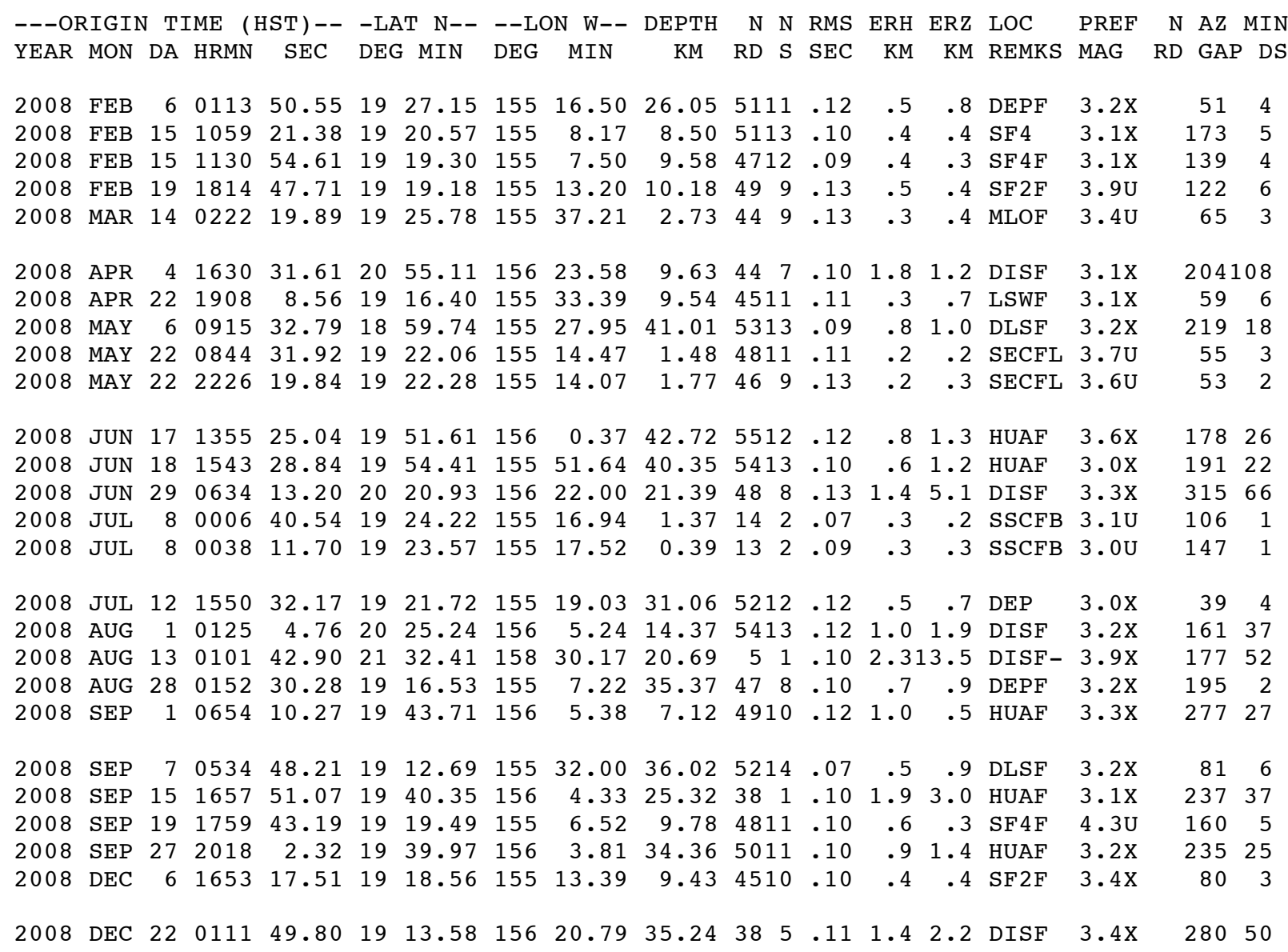



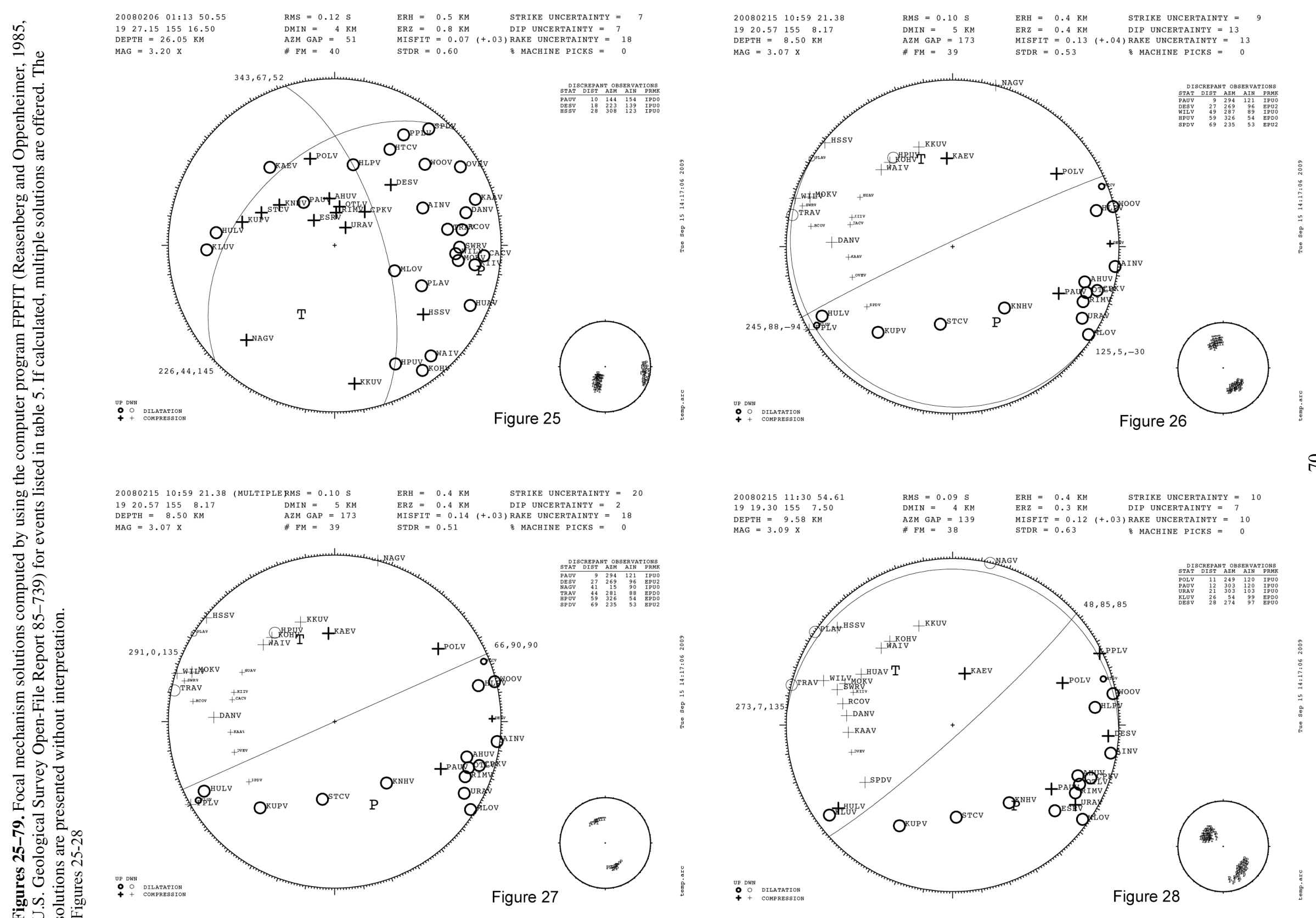

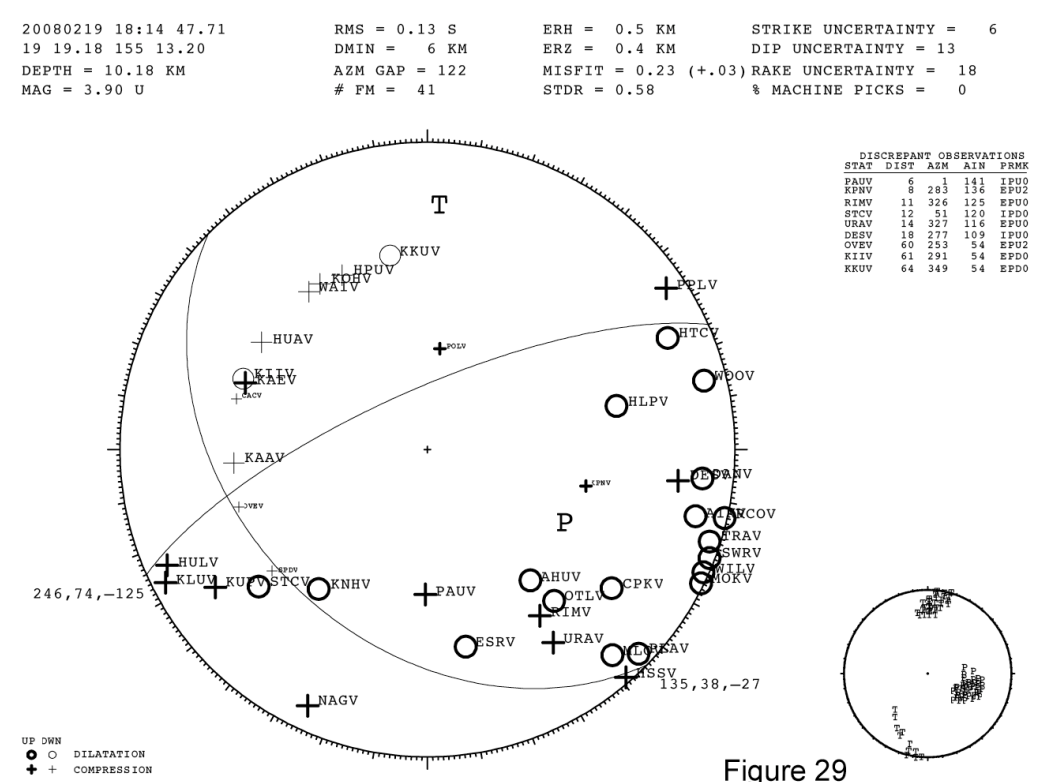

Figure 29

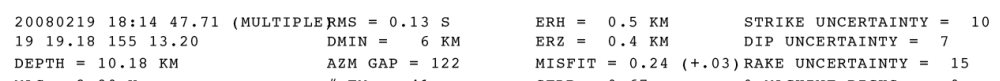

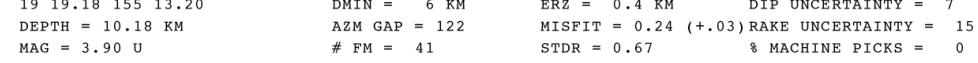

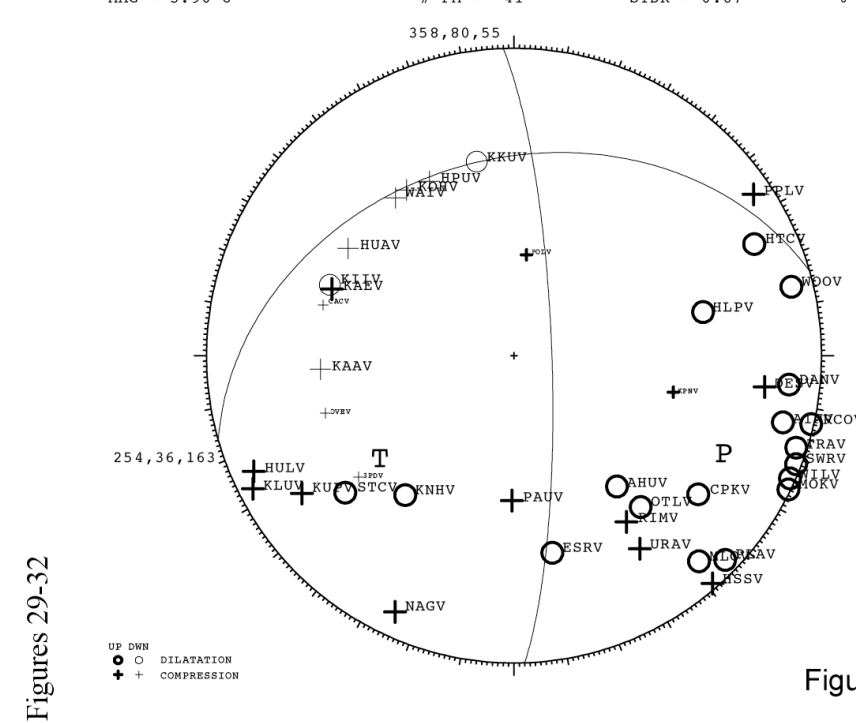

Figure 3
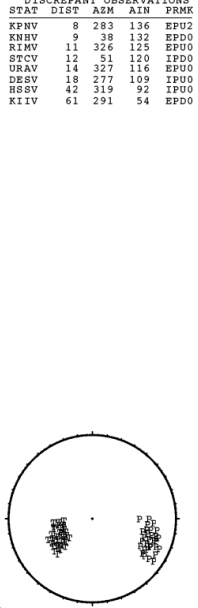

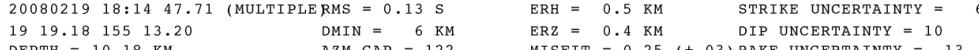

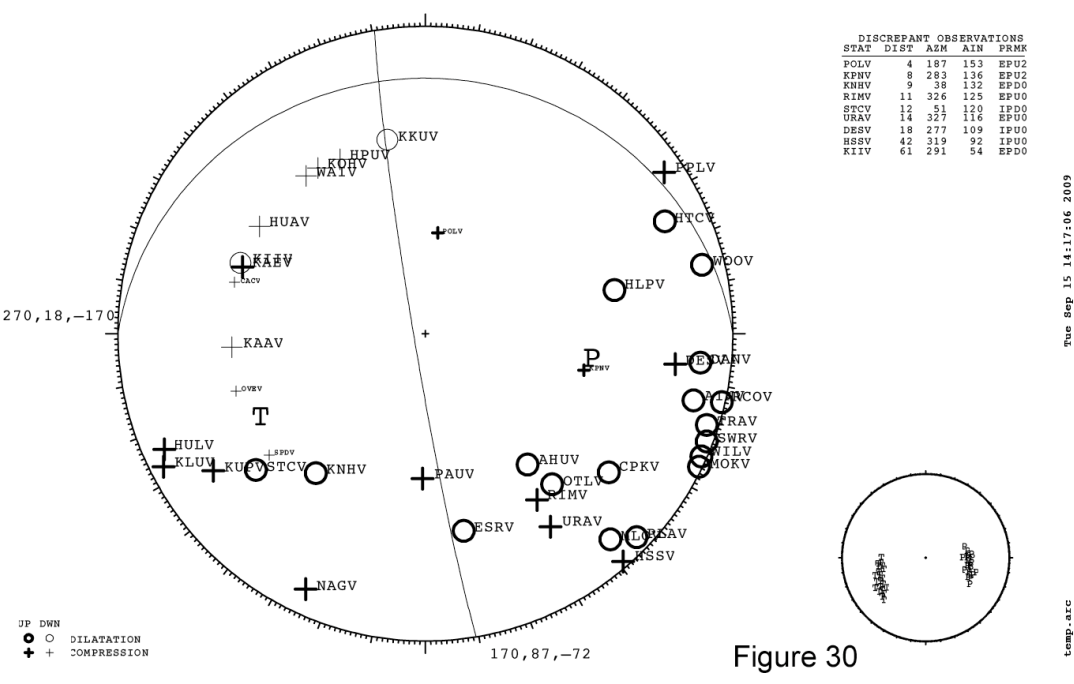

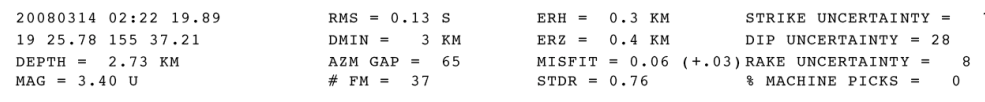
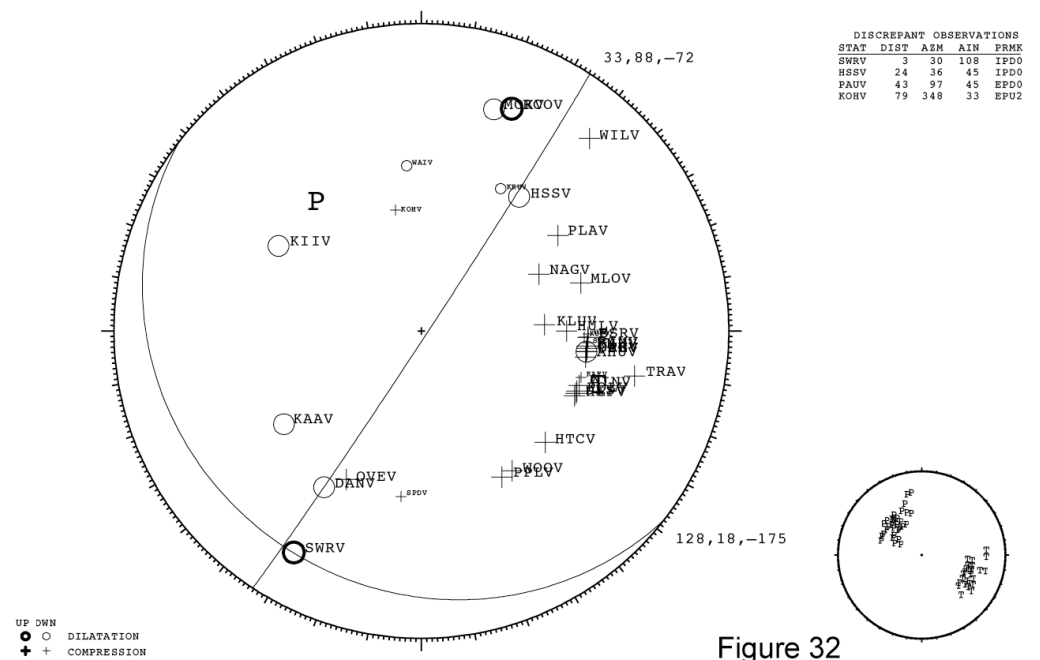

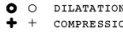

Figure 32

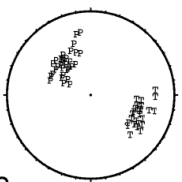

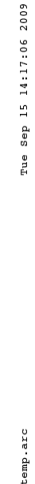



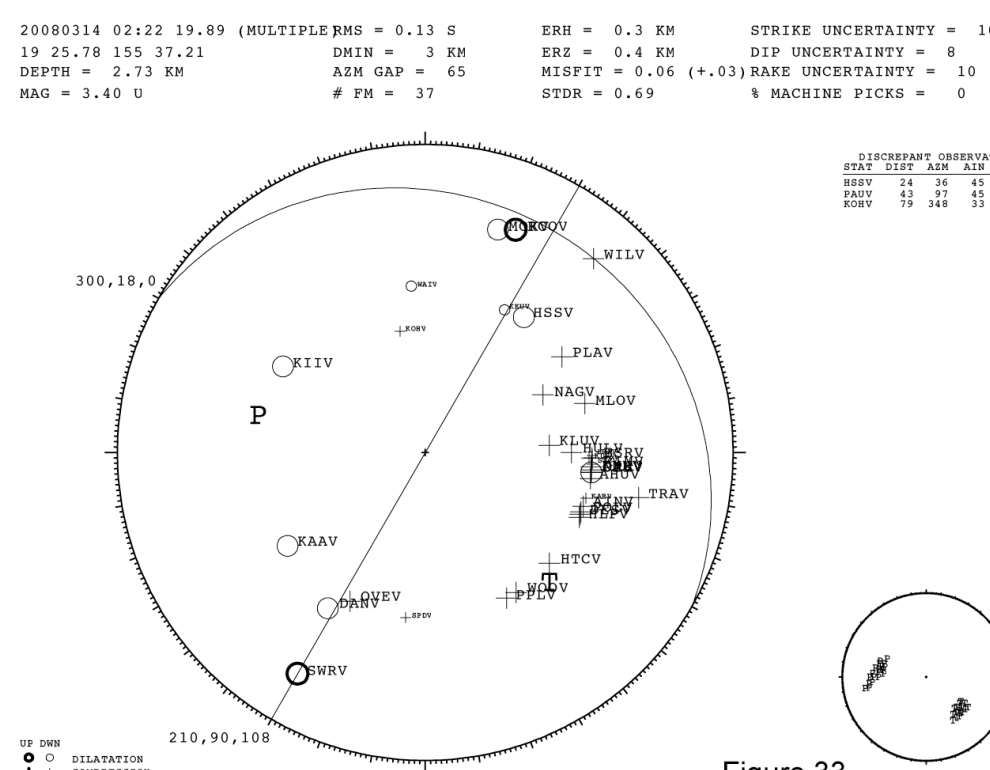

Figure 33
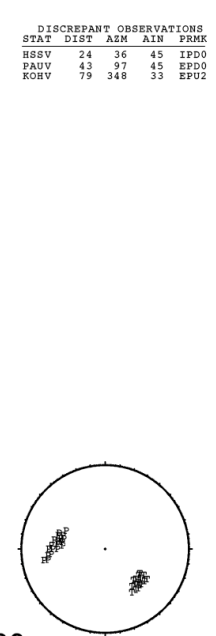

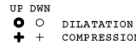

$2008040416: 3031.61$
2055.1115623 .58
DEPTH $=9.63 \mathrm{KM}$

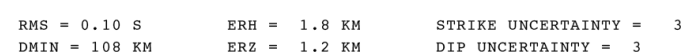

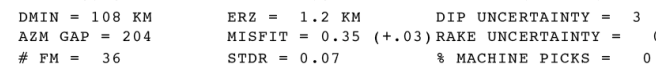
$M A G=3.09$

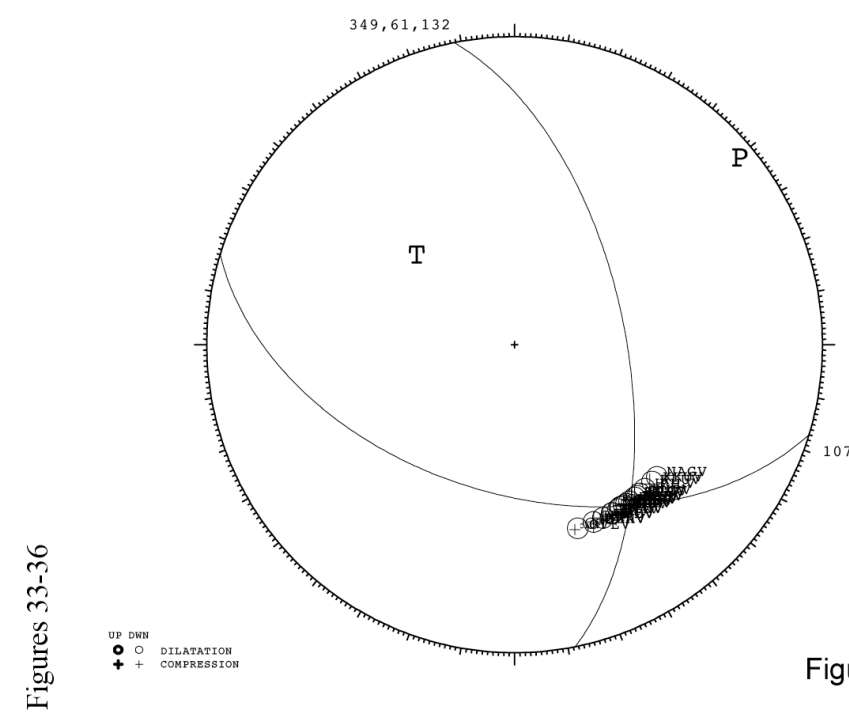

Figure 35

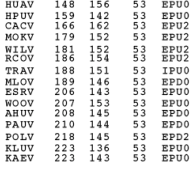

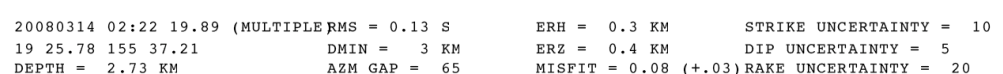

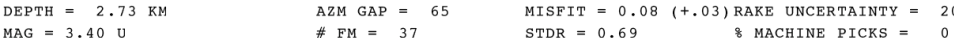

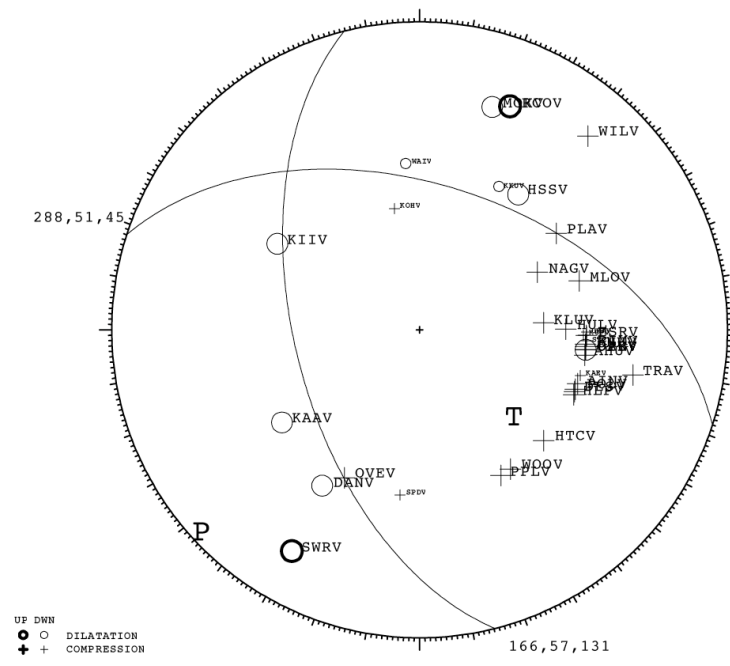

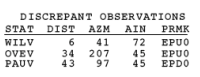

Figure 34

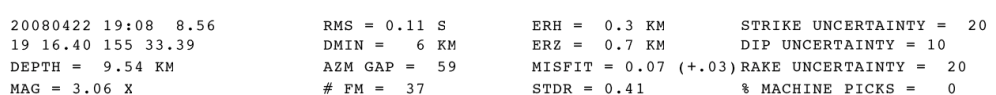

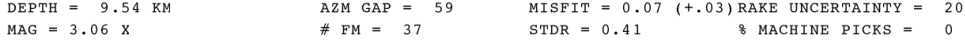
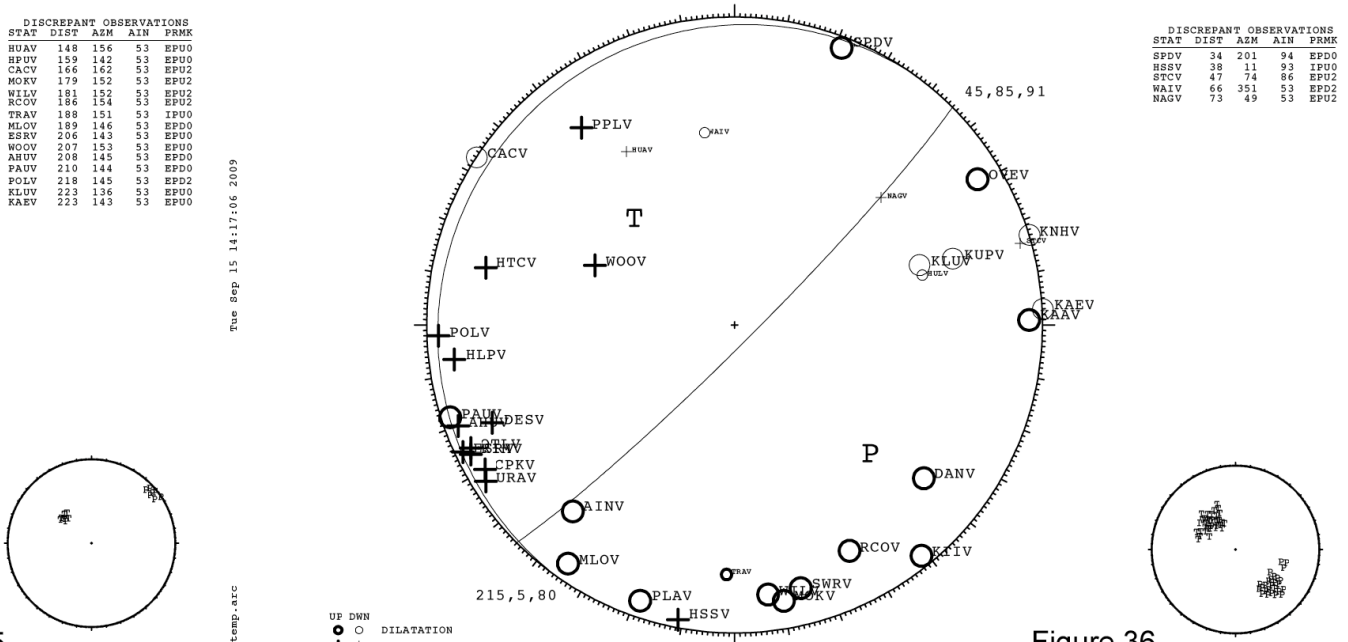

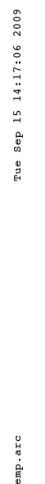

$N$

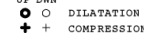

Figure 36 

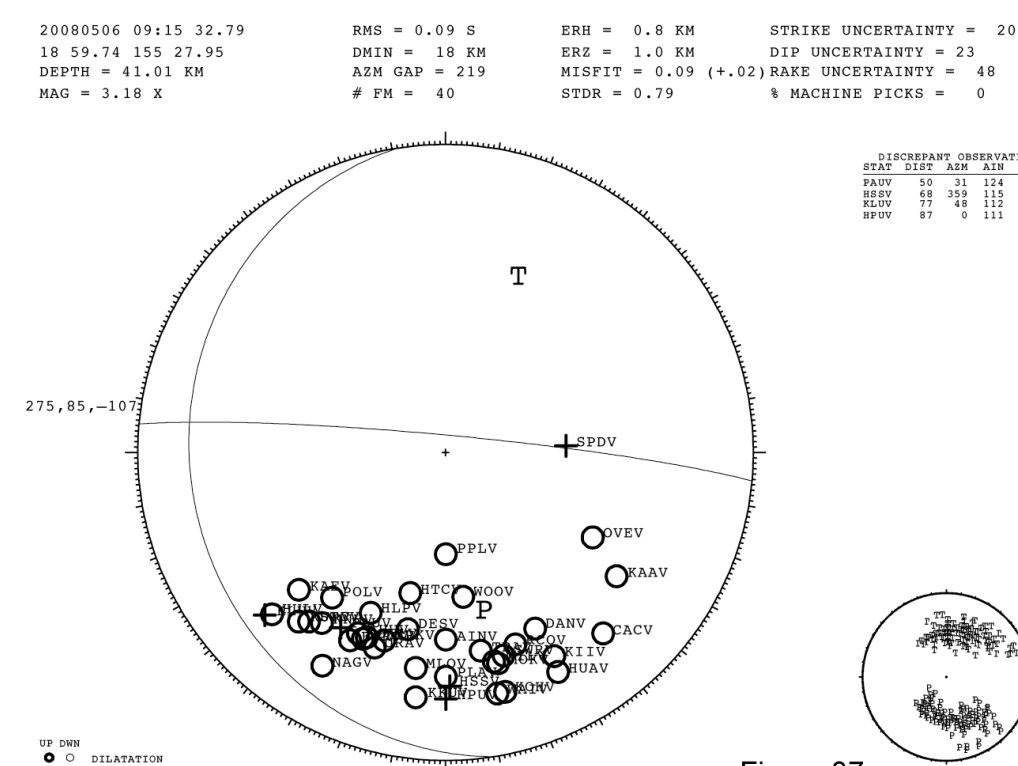

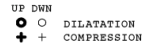

Figure 37

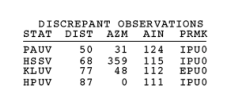

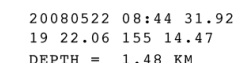$$
\text { DEPTH }=1.48 \mathrm{KM}
$$

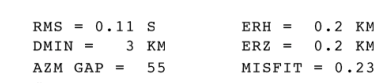$$
\begin{aligned}
& \text { MISFTT }=0.23 \\
& \text { STDR }=0.62
\end{aligned}
$$

STRIKE UNCERTAINTY $=180$ RARE UNCERTAINTYY $=10$

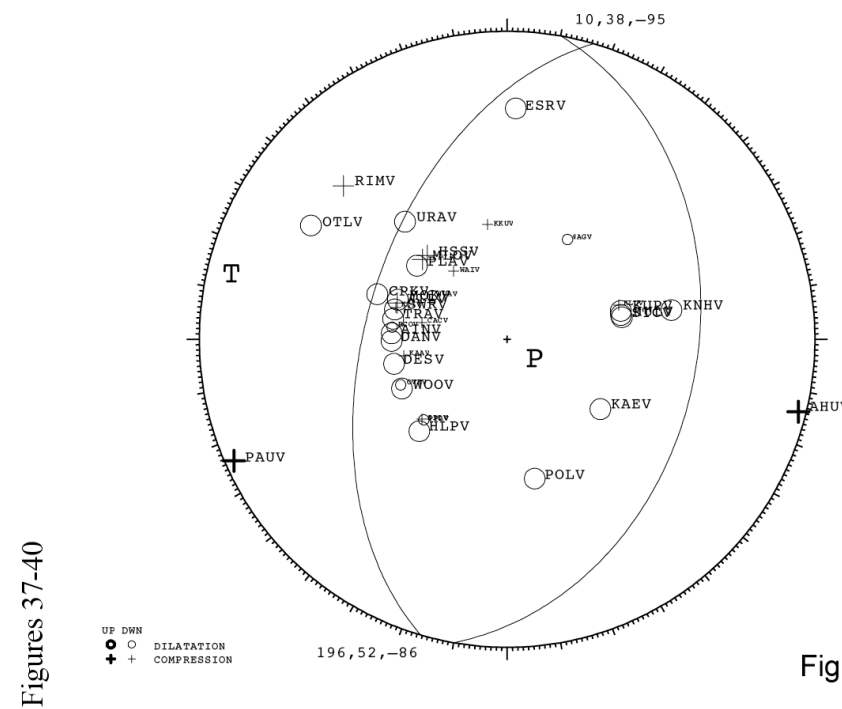

Figure 39

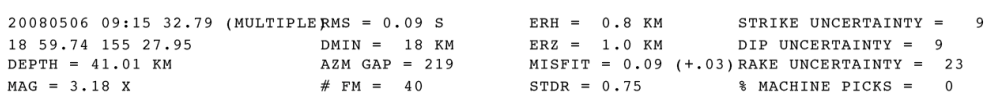

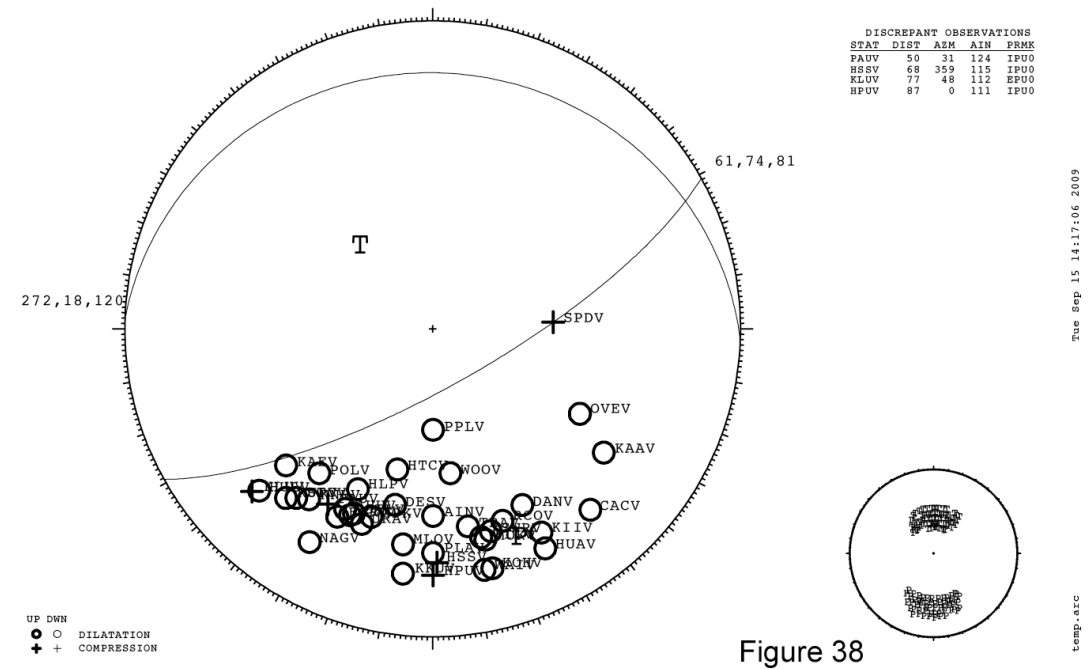

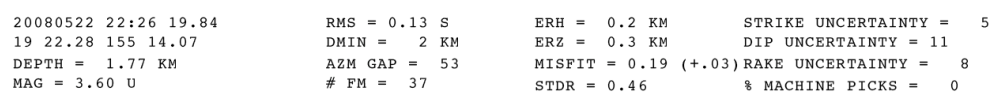

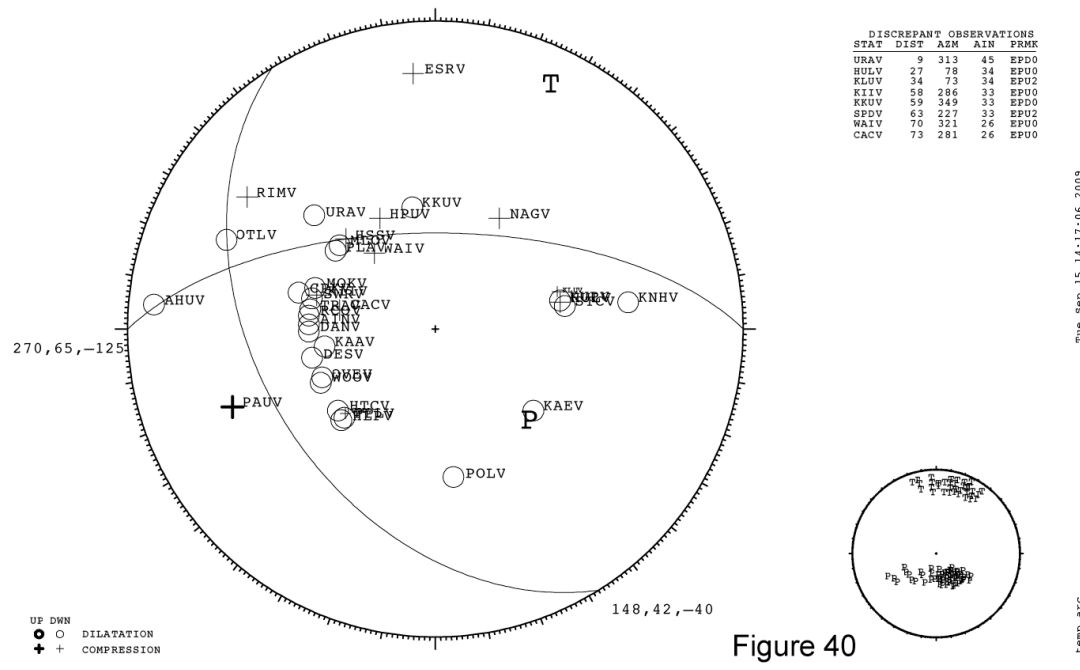



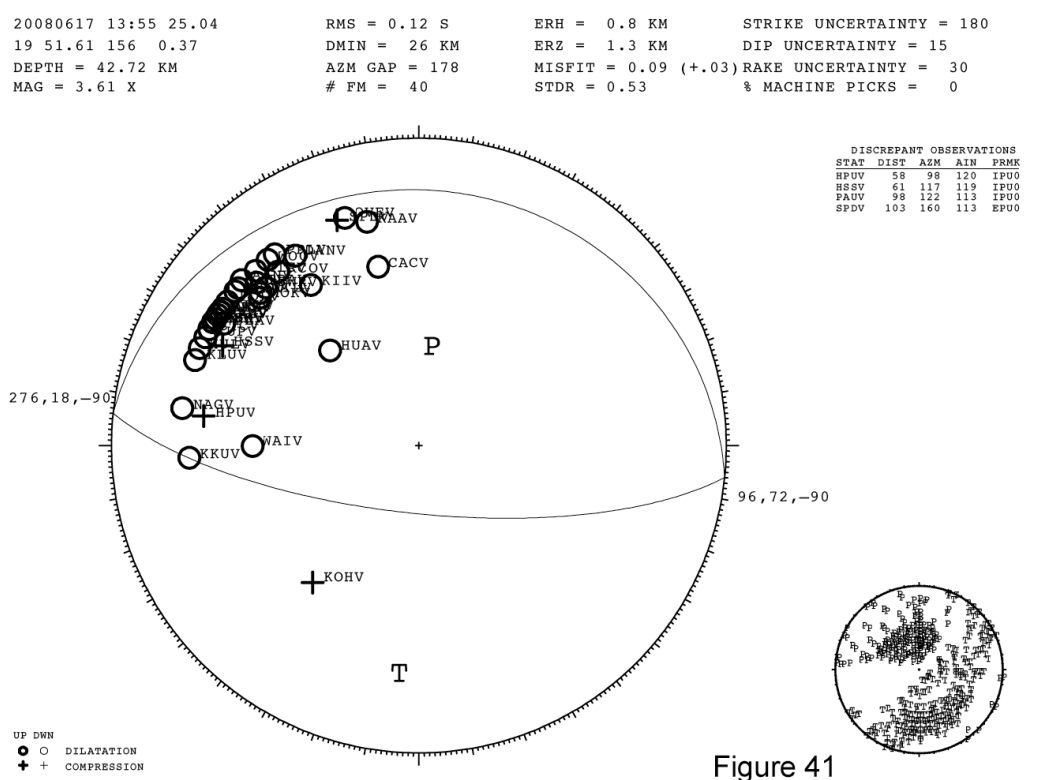

Figure 41

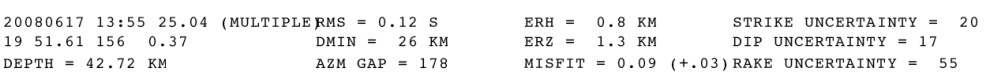

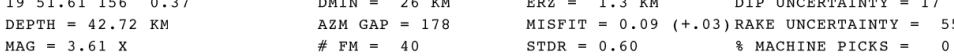

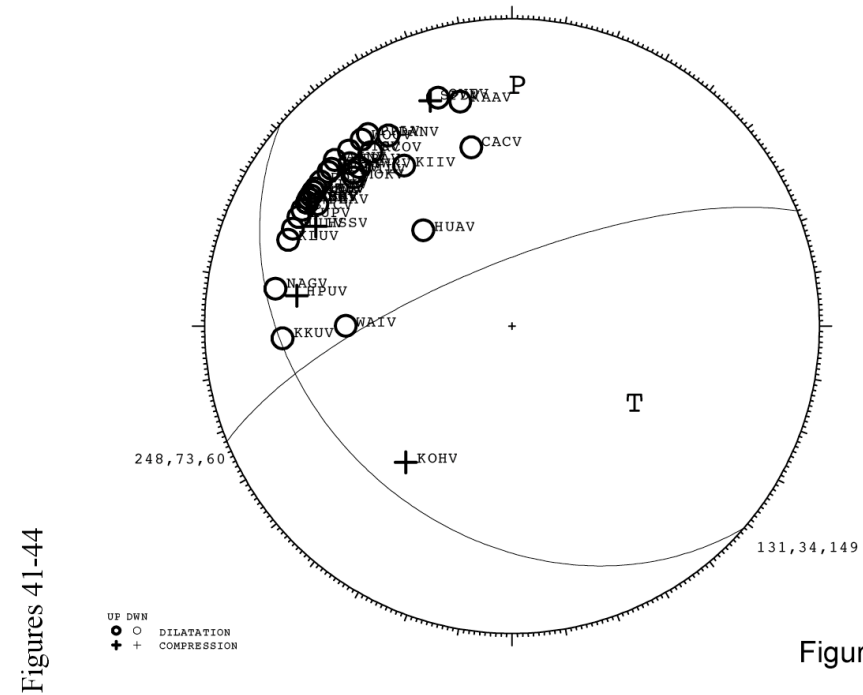

Figure 43 $\begin{array}{lllll}20080617 & 13: 5525.04 & \text { MULTIPLERMS }=0.12 \mathrm{~s} & \text { ERH }=0.8 \mathrm{KM} & \text { STRIKE UNCRRTAINTY }= \\ 1951.611560 .37 & \text { DMIN }=26 \mathrm{KM} & \text { ERZ }=1.3 \mathrm{KM} & \text { DIP UNCERTAINTY }=10\end{array}$ MADOR $=0.77$ (1.02) DaP

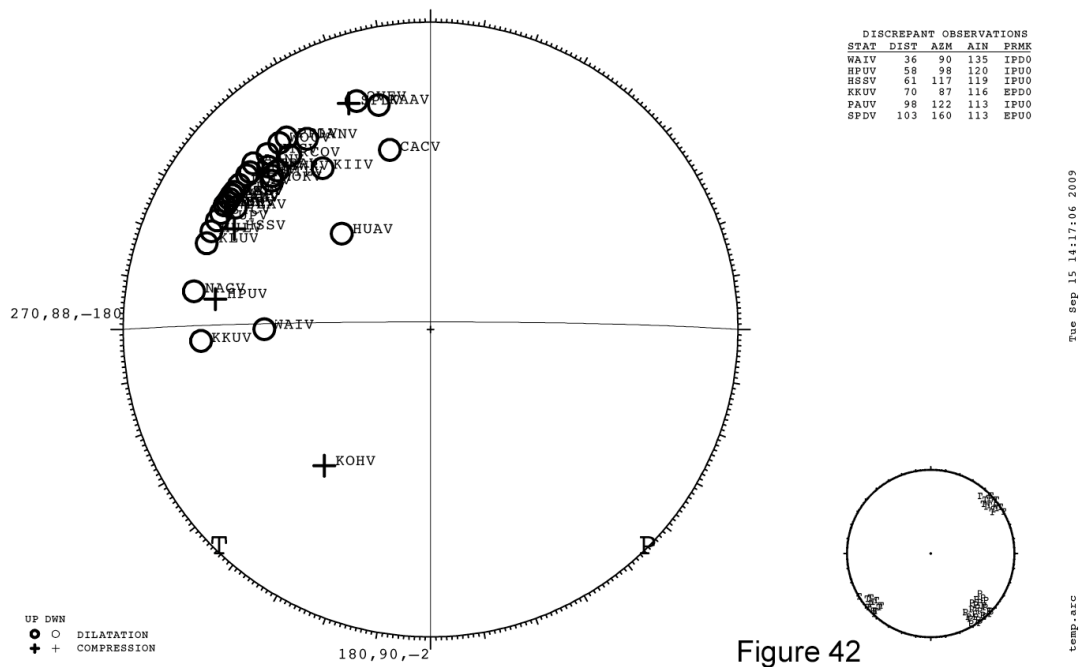

$2008061713: 5525.04$ (MULTTPLER RHS $=0.12 \mathrm{~s} \quad$ ERH $=0.8 \mathrm{KM} \quad$ STRIKE UNCRRTAINTY $=$

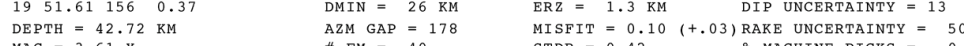

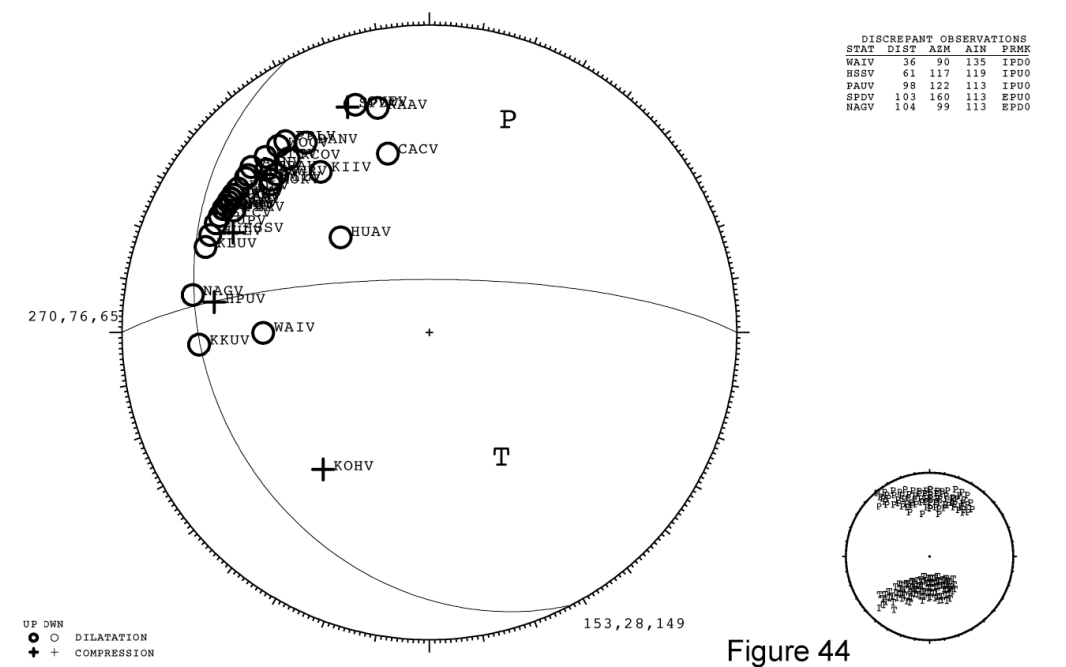


$2008061815: 4328.84$
1954.4115551 .64

DEPTH $=40.35 \mathrm{~K}$

RMS $=0.10 \mathrm{~s}$

$\mathrm{DMIN}=22$
AZM GAP

$\mathrm{ERH}=0.6 \mathrm{KH}$

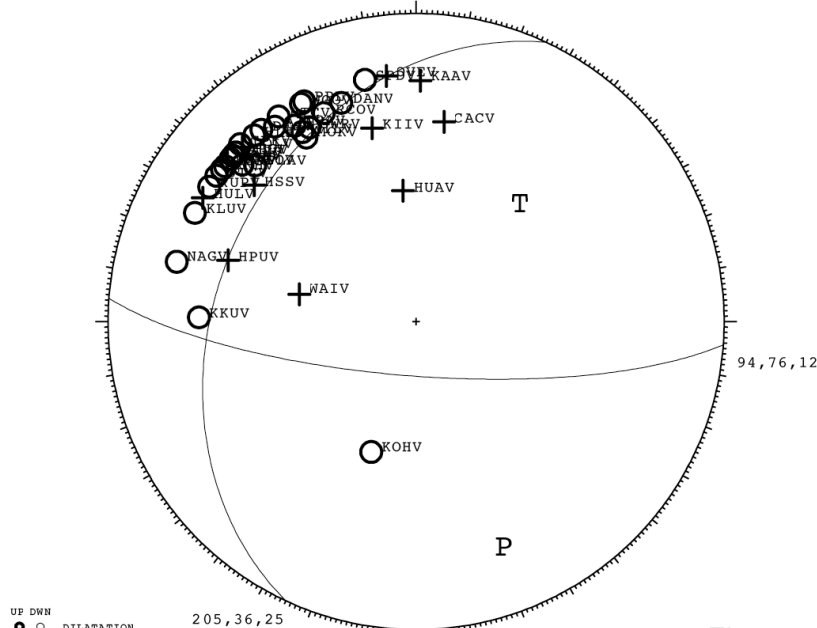

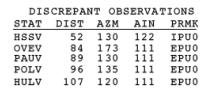

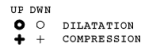

Figure 45

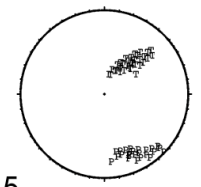

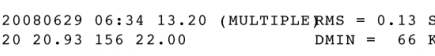
AZM GAP $=315$

ERH $=1.4 \mathrm{KM} \quad$ STRIRE UNCERTAINTY $=16$ DEPTH $=21.39$ $\begin{aligned} \text { DIP UNCERTAINTY } & =2 \\ \text { MISFIT } & =0.06(+.02) \text { RAKE UNCERTAINTY }\end{aligned}=10$
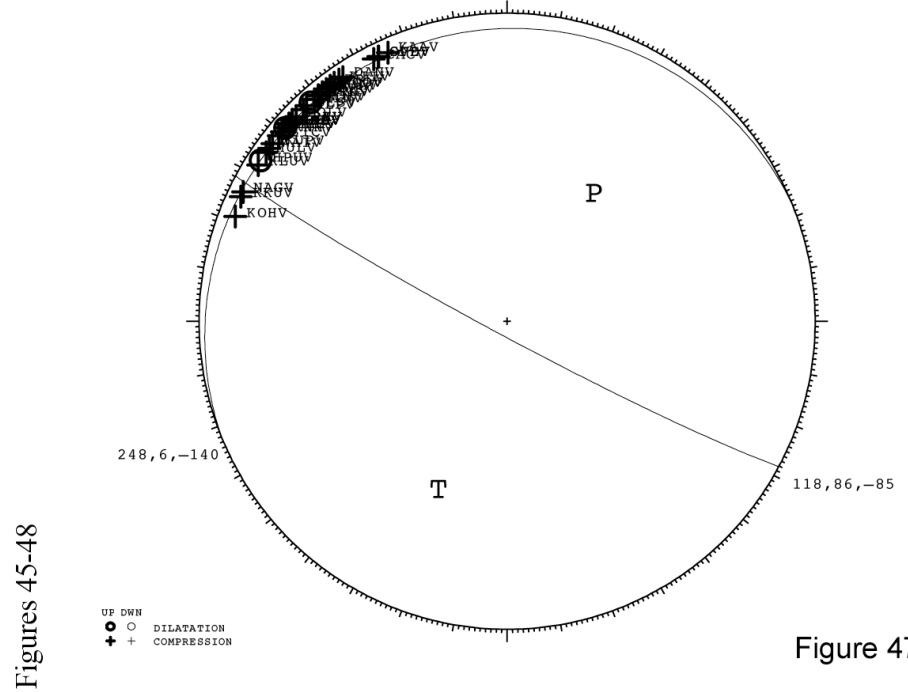

Figure 47
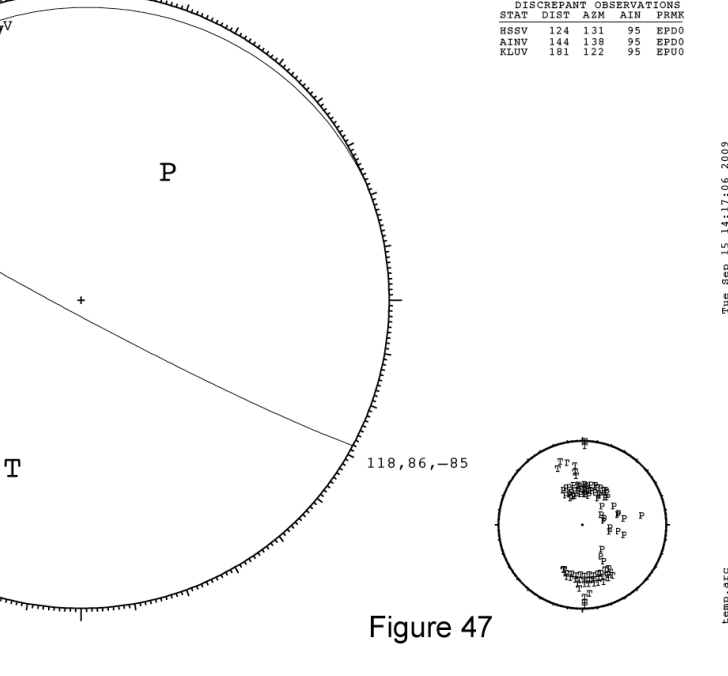

$20080629 \quad 06: 34 \quad 13.20$
$2020.93 \quad 15622.00$ AZM GAP $=31$

$\mathrm{BRH}=1.4 \mathrm{KM}$ STH UNCERATNY $=23$

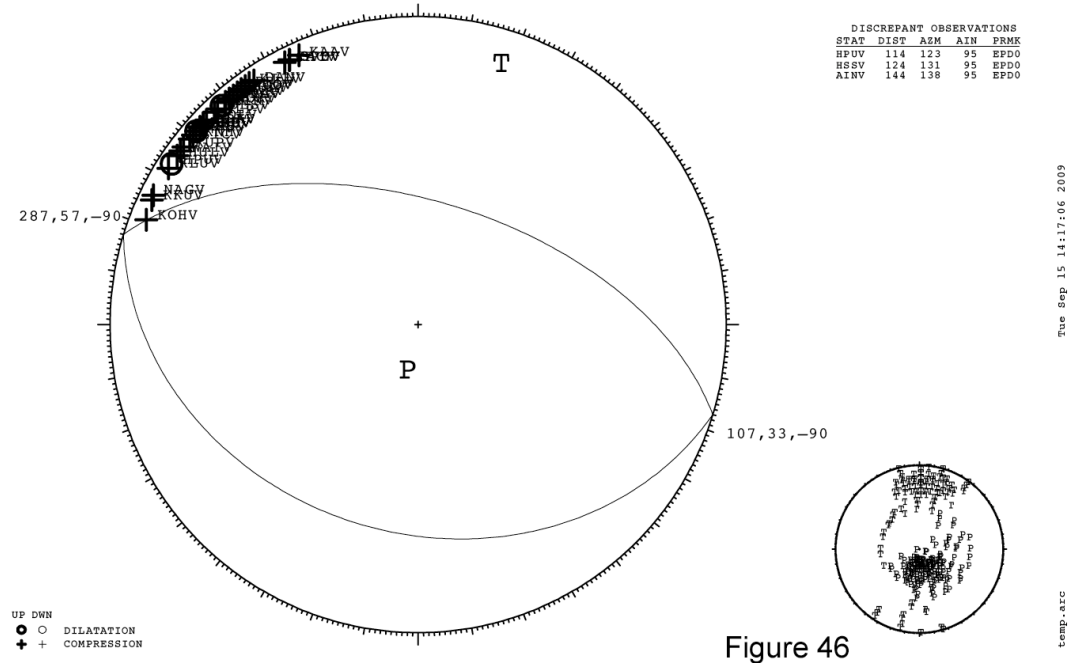

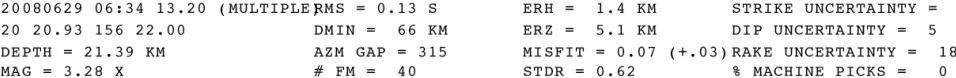

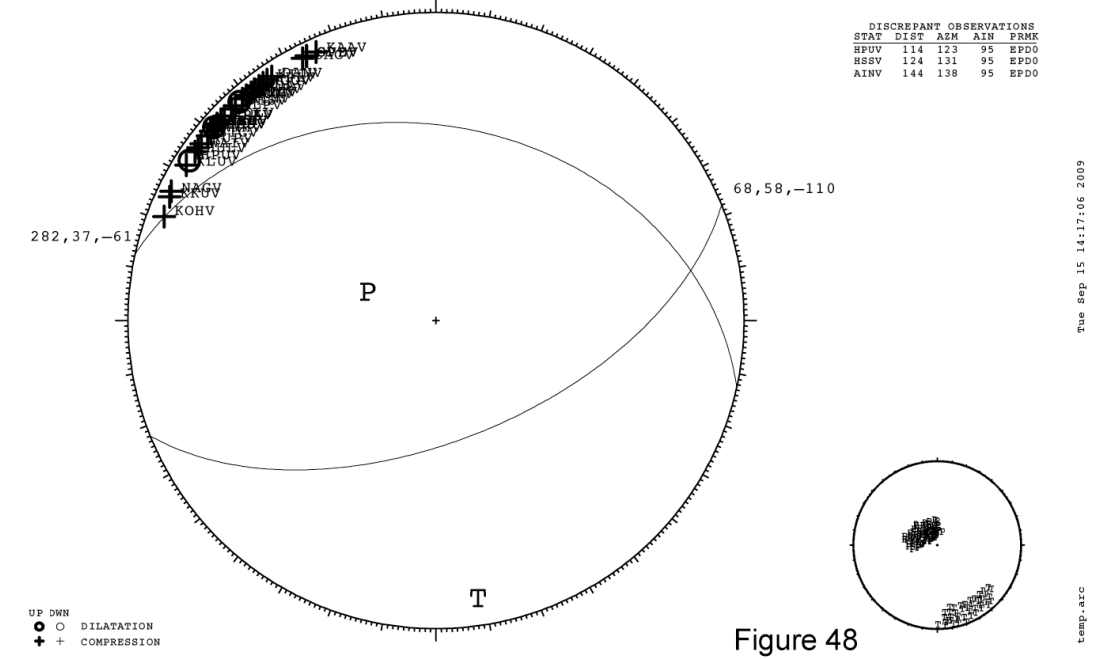



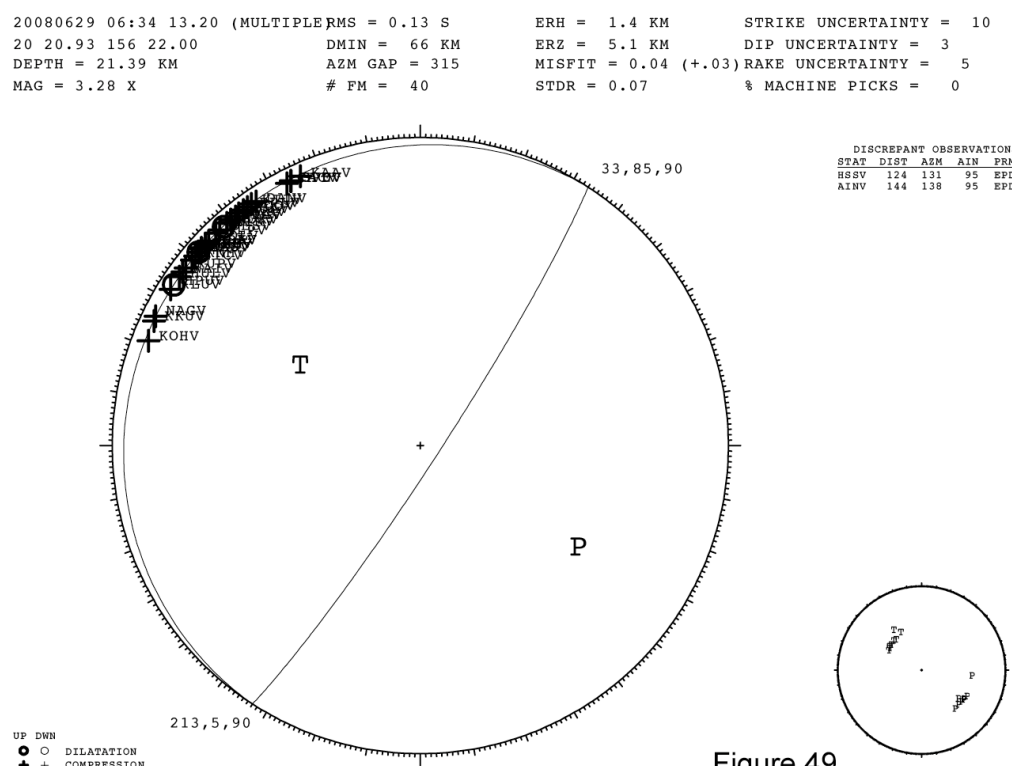

Figure 49
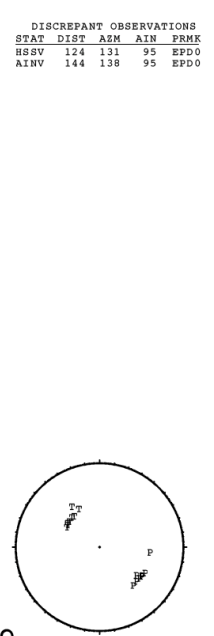

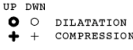

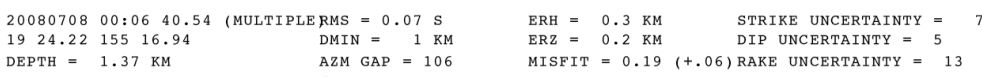

DEPTH $=1.37 \mathrm{KM}$
MAG $=3.10 \mathrm{U}$

AZM GAP $=10$

WISFIT $=0.19(+.06)$ RAKE UNCERTAINTY $=$

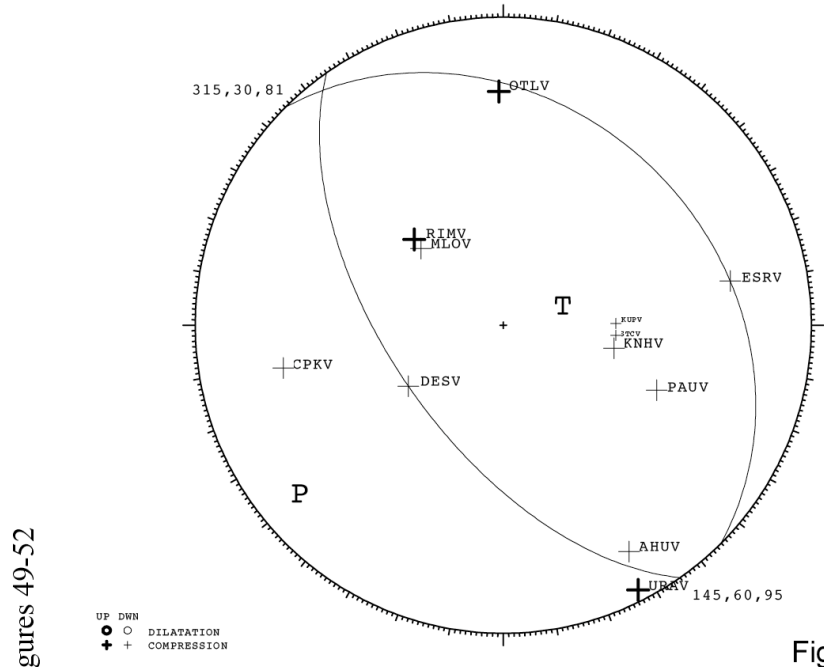

Figure 51
$20080708 \quad 00: 06 \quad 40.54$ 1924.2215516 .94
DEPTH $=1.37 \mathrm{KM}$ DEPTH $=1.37$
MAG $=3.10 \mathrm{U}$ DMIN $=1 \mathrm{KM}$ $\angle \mathrm{FM}=12$ 8 MACHINE PICKS $=$

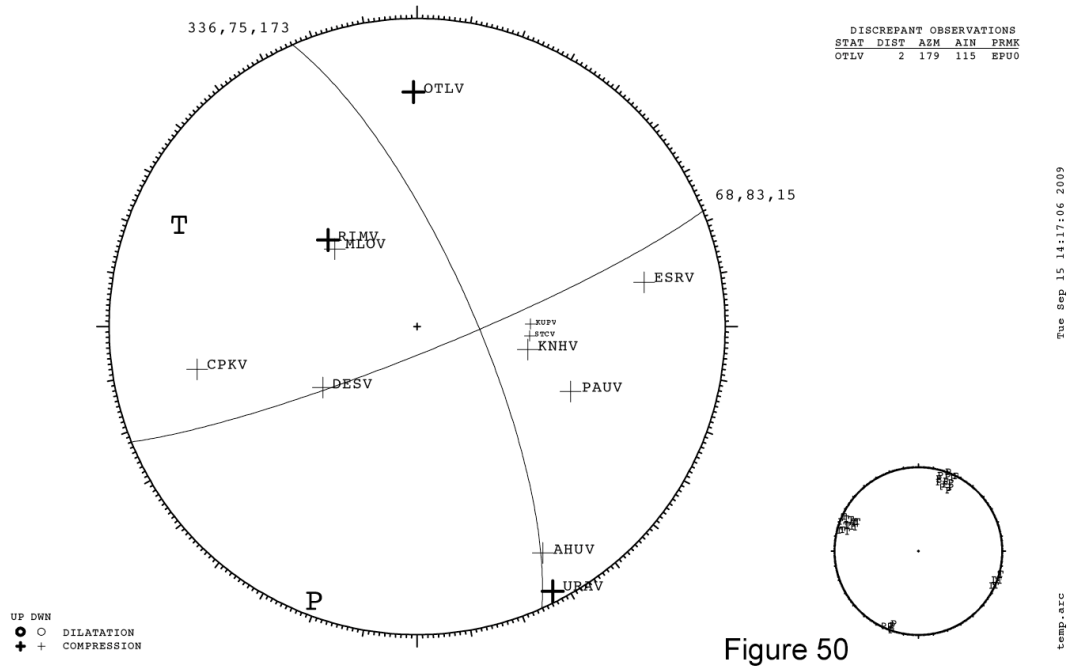

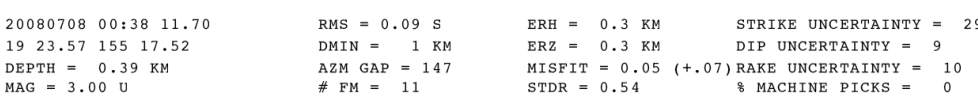

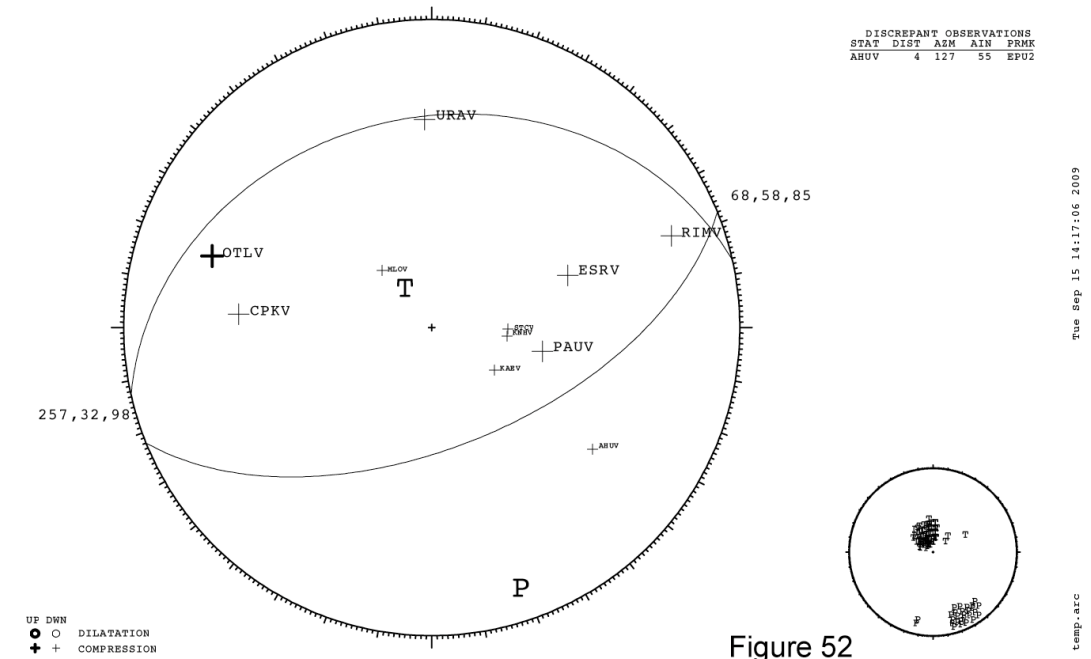



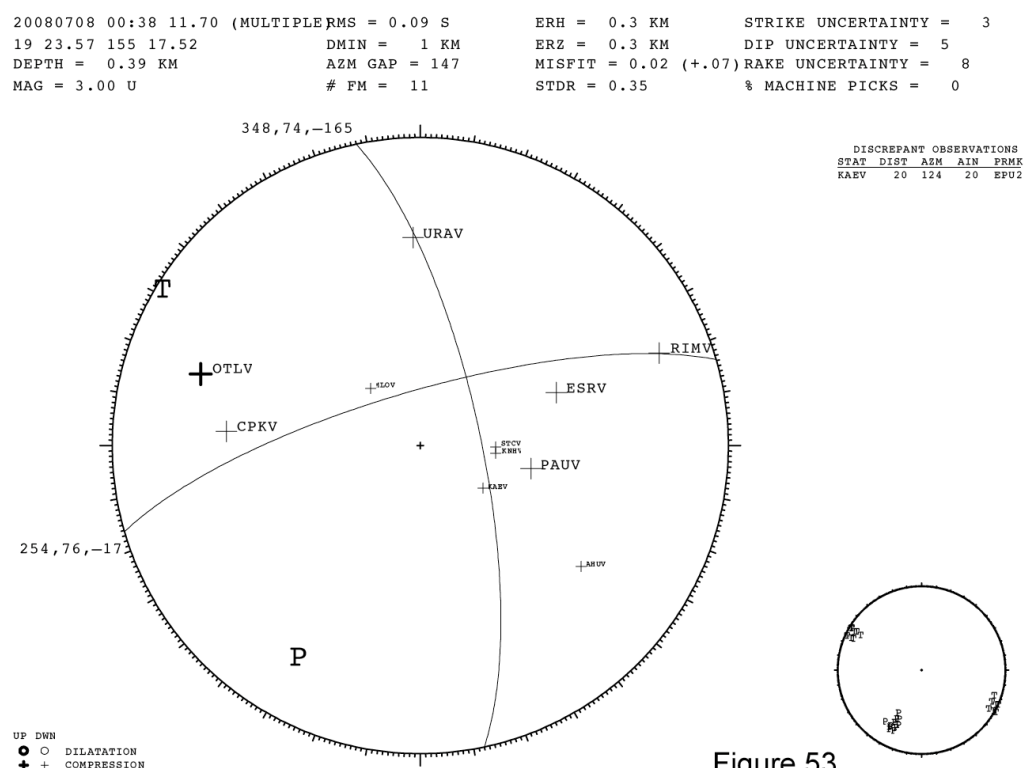

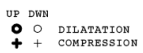

Figure 53

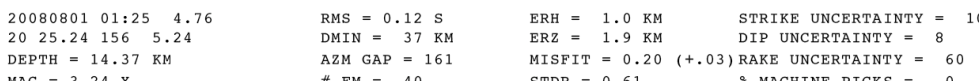
DEPTH $=14.37 \mathrm{KM}$ AZM GAP $=16$ MISFIT $=0.20(+.03)$ RAKE UNCERIAINTY $=$

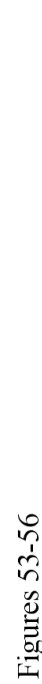

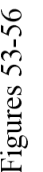

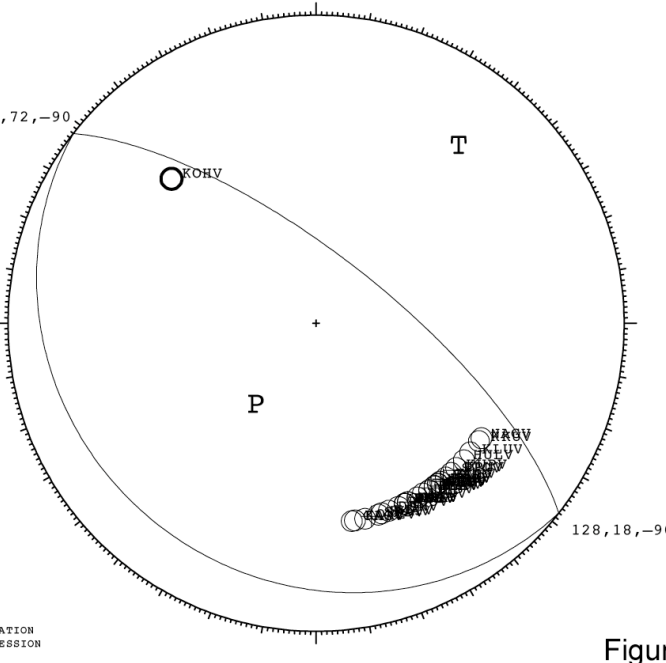

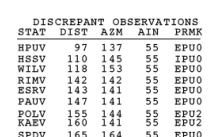

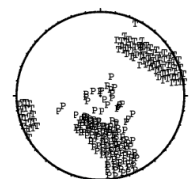

$20080712 \quad 15: 50 \quad 32.17$ 1921.7215519 .03
DEPTH $=31.06 \mathrm{KM}$ DMIN $=4.45$

BRH $=0.5 \mathrm{KM}$ STRIKE UNCERTAINTY =

RARE UNCERTATNTY $=2$
8 MACHINE PICKS $=0$
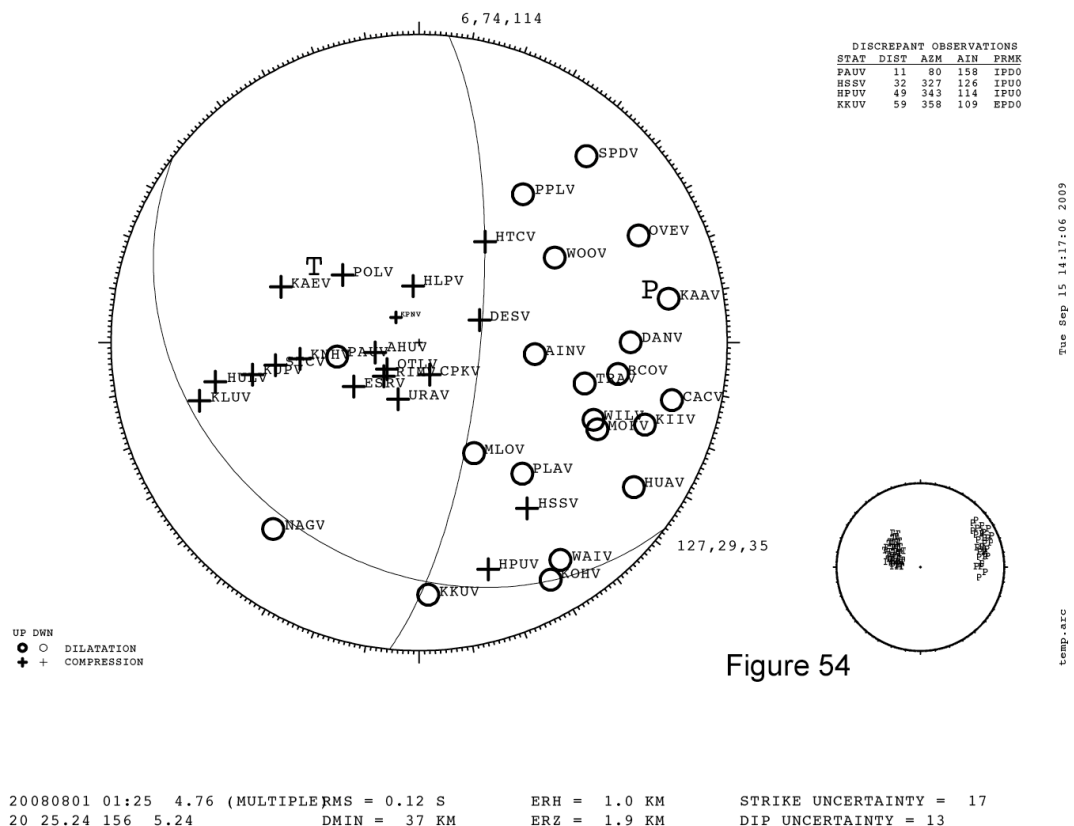

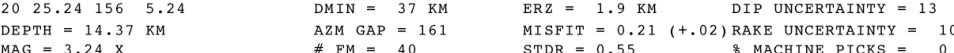

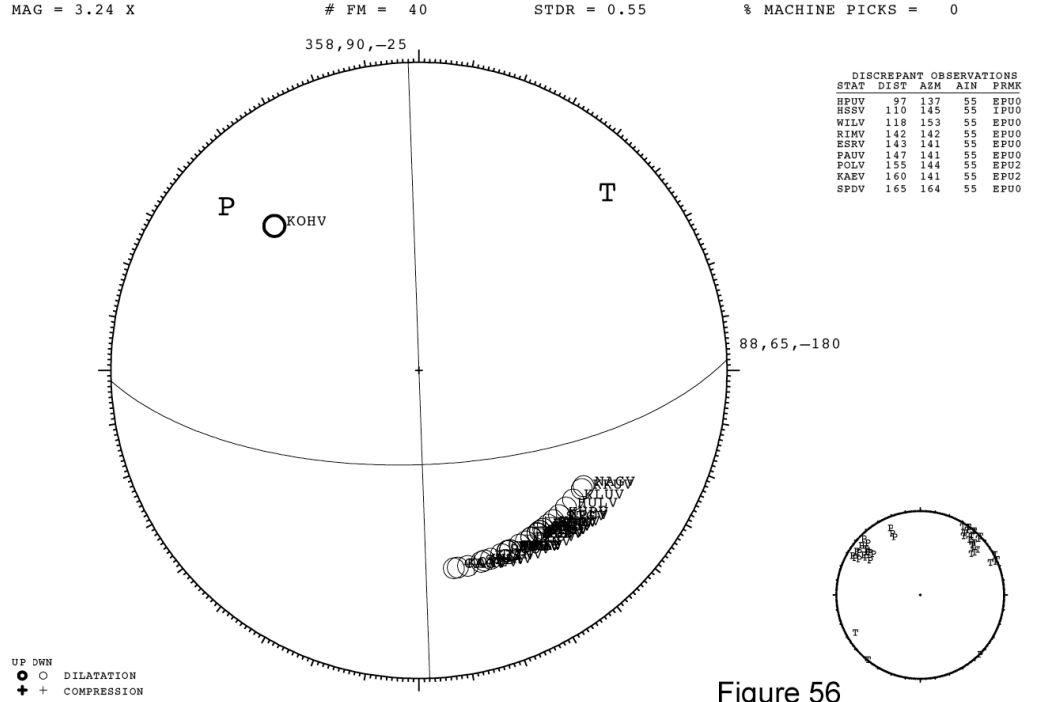




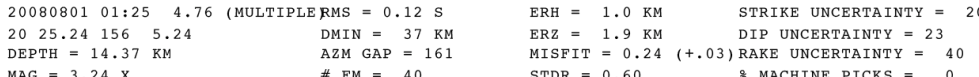

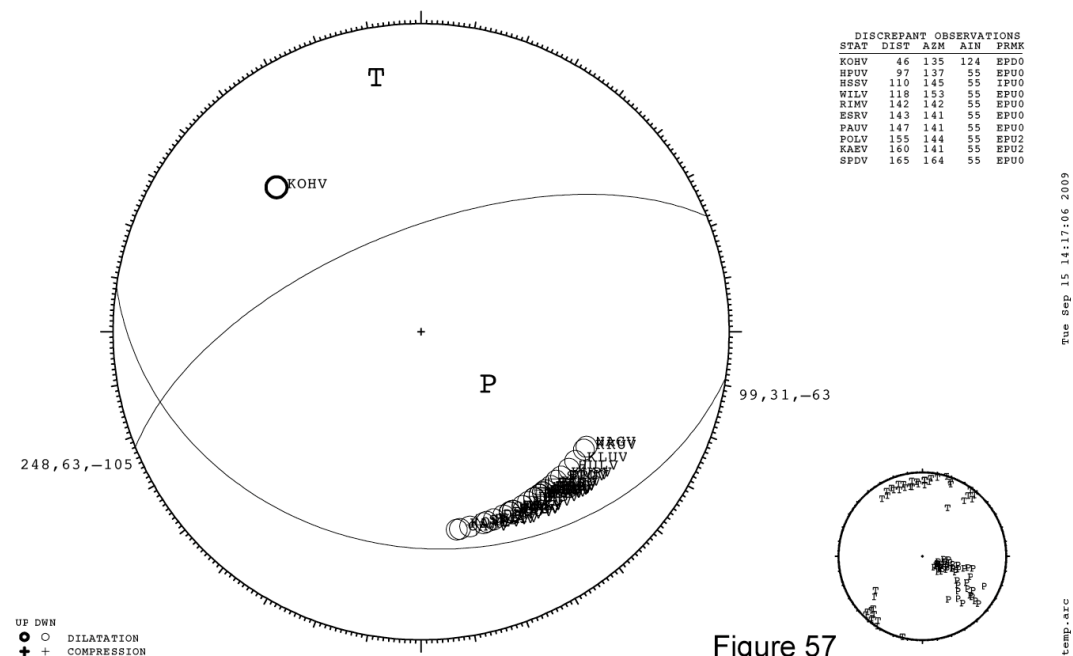

Figure 57

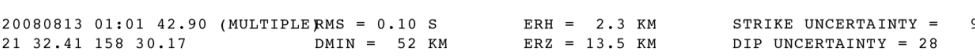

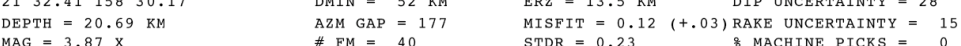

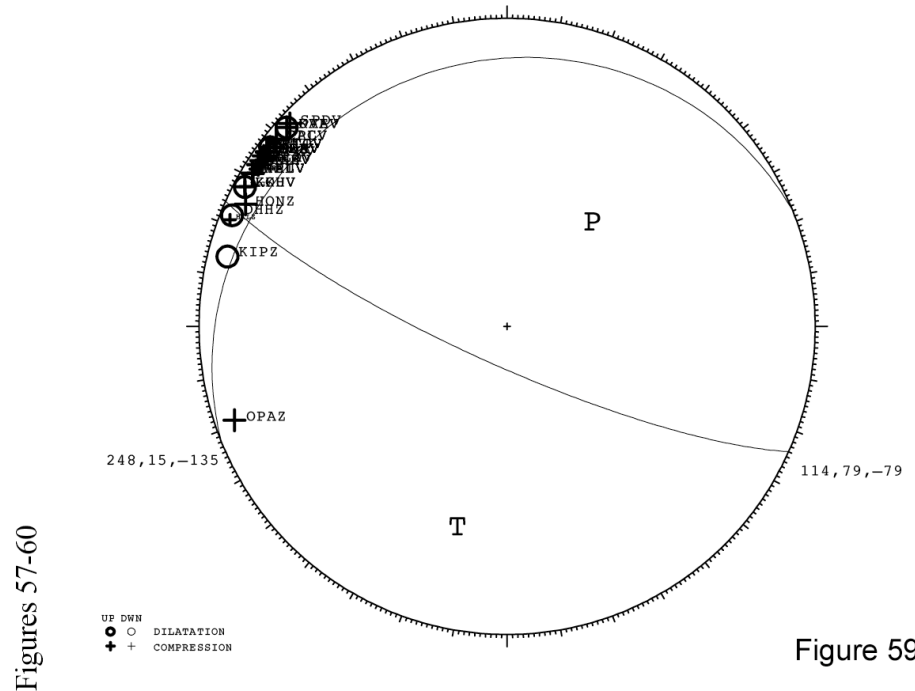

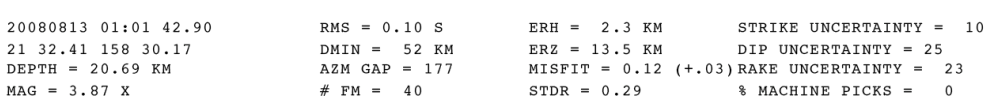

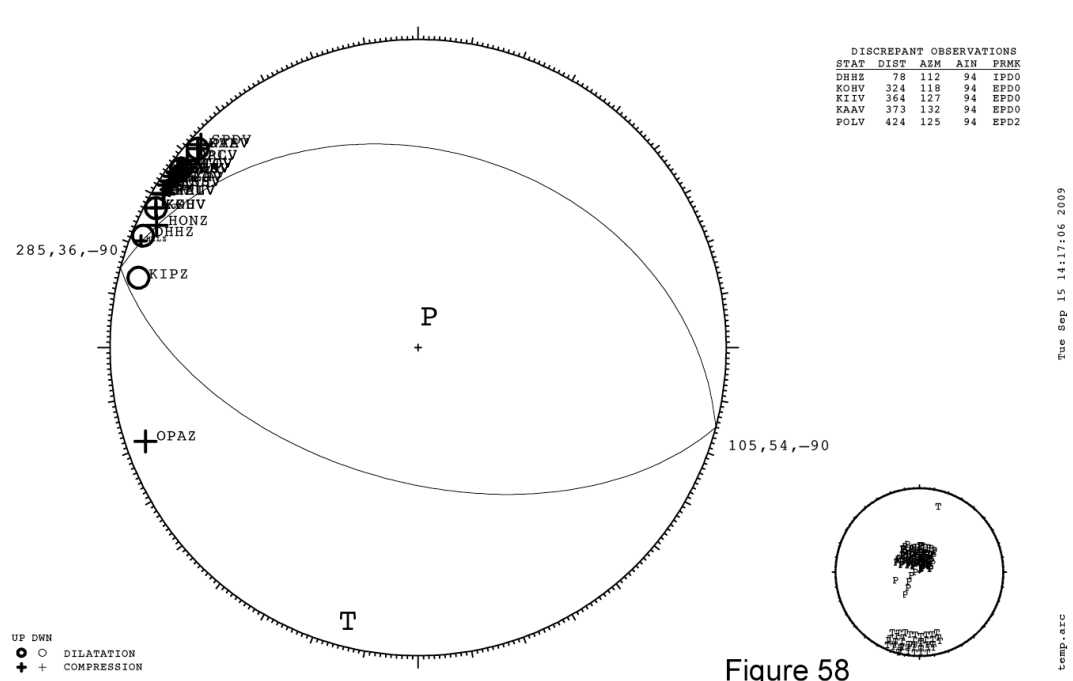
$\begin{array}{lclll}20080813 & 01: 0142.90 & \text { MULTIPLERMS }=0.10 \mathrm{~s} & \text { ERH }=2.3 \mathrm{KM} & \text { STRIRE UNCERTA INTY }=10 \\ 2132.415830 .17 & \text { DMIN }=52 \mathrm{kM} & \text { ERZ }=13.5 \mathrm{kM} & \text { DIP UNCERTAINTY }=3\end{array}$

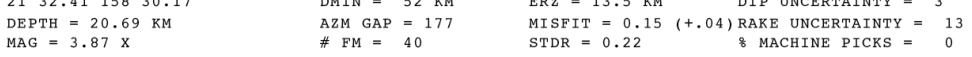

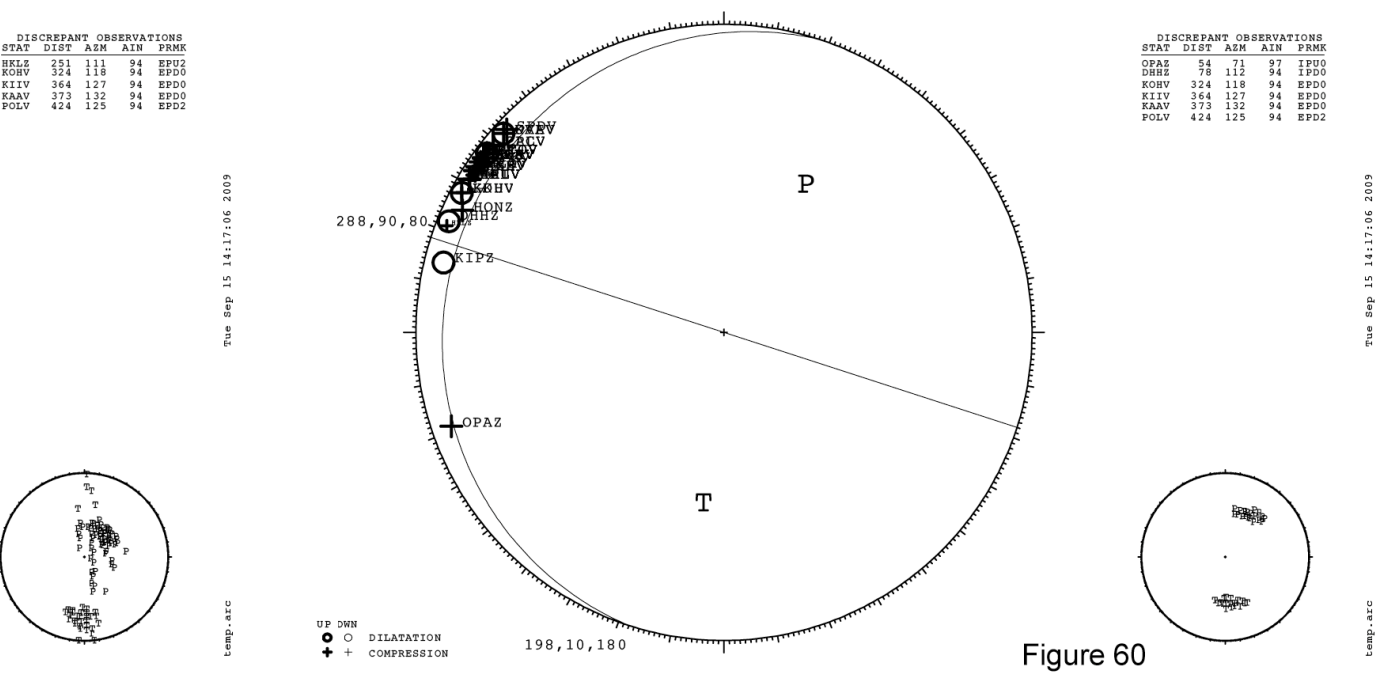



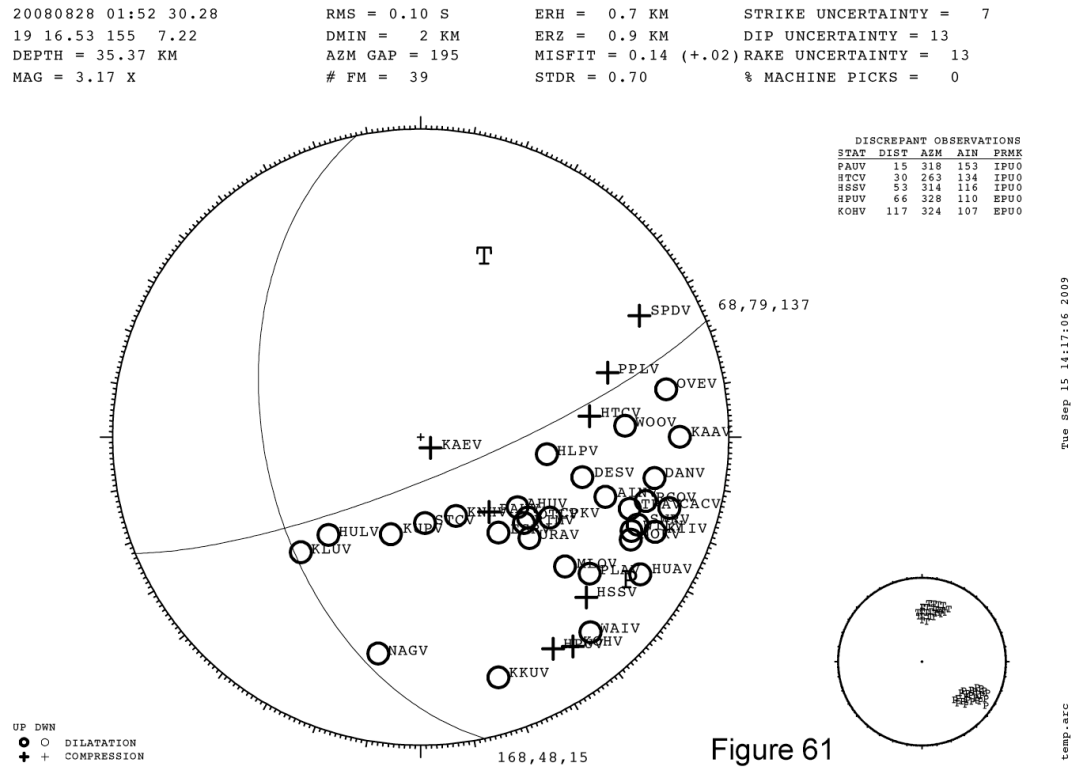

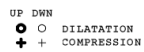

Figure 61

$2008082801: 5230.28$ (MULTIPLERMS $=0.10 \mathrm{~s} \quad$ ERH $=0.7 \mathrm{kM} \quad$ STRIKE UNCRRTAINTY $=10$

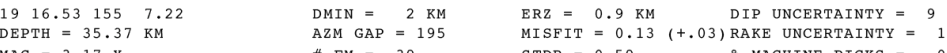
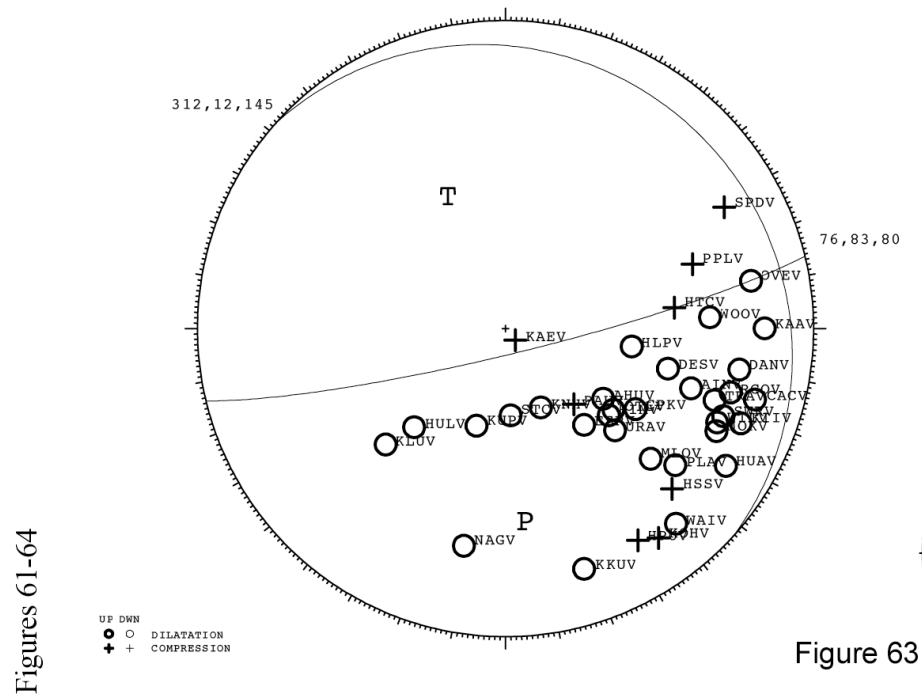

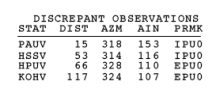

Figure 63

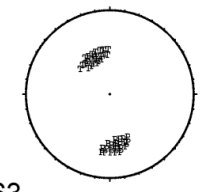

$2008082801: 5230.28$ (MUL,TIPLERMS $=0.10 \mathrm{~s}$

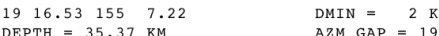
* $\mathrm{FM}=39$

BRH $=0.7 \mathrm{KM}$

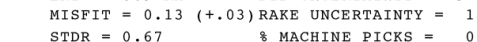

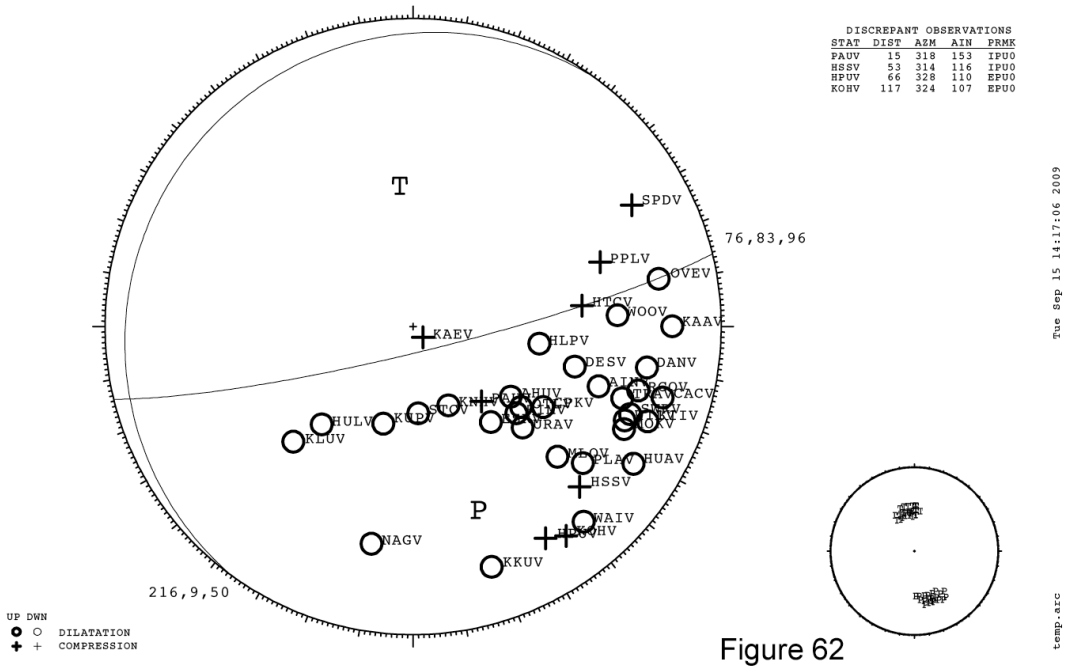

શ

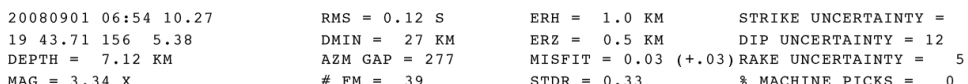

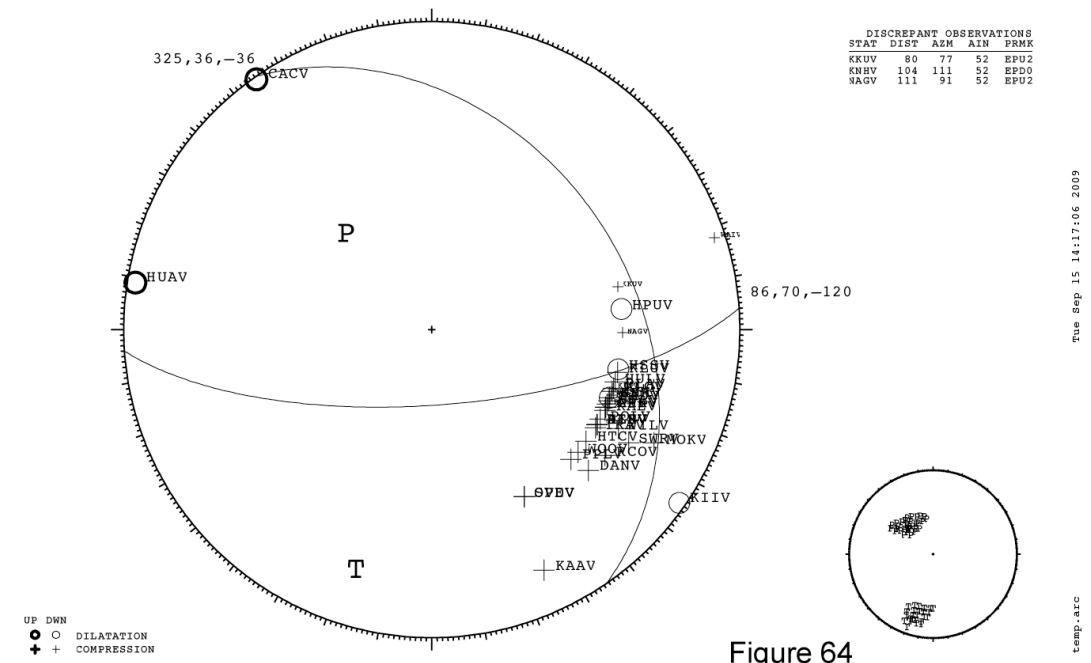



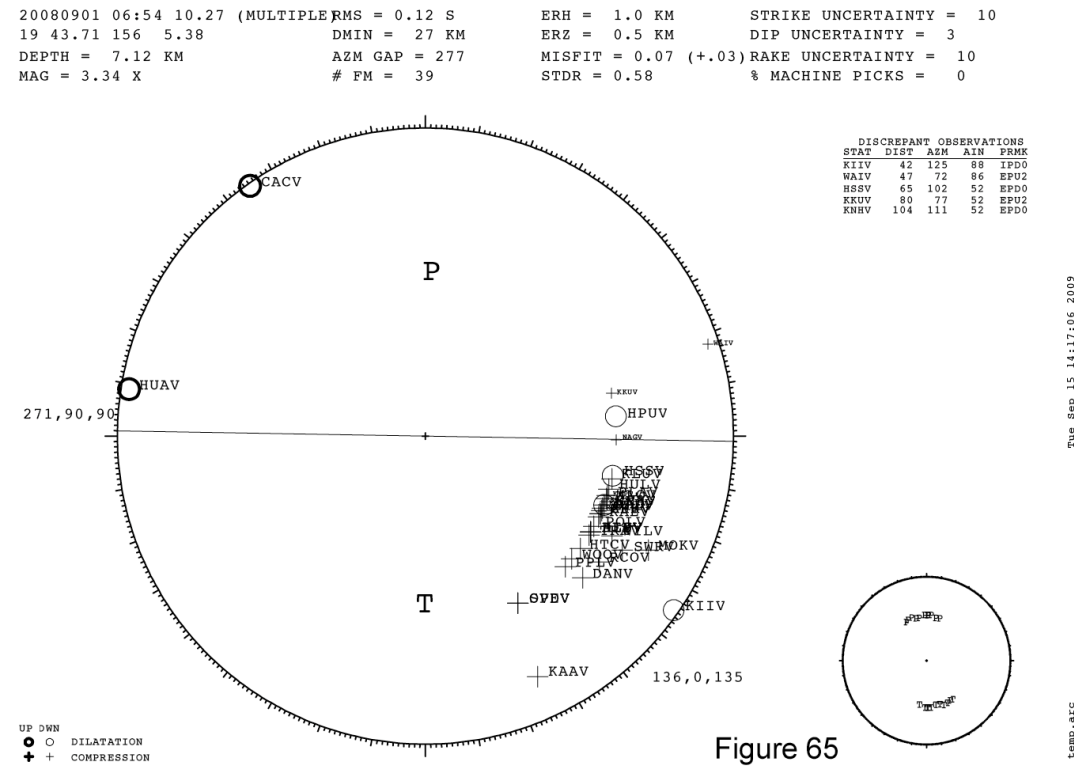

Figure 65

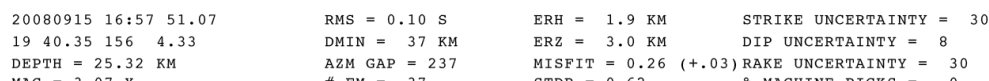

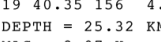
$\begin{array}{lcc}\text { AZM GAP }=237 & \text { MISFIT }=0.26 & (+.03) \text { RARE UNCERTAINTY }=30 \\ \# \text { FM }=37 & \text { STDR }=0.62 & \text { B MACHINE PICKS }=30\end{array}$
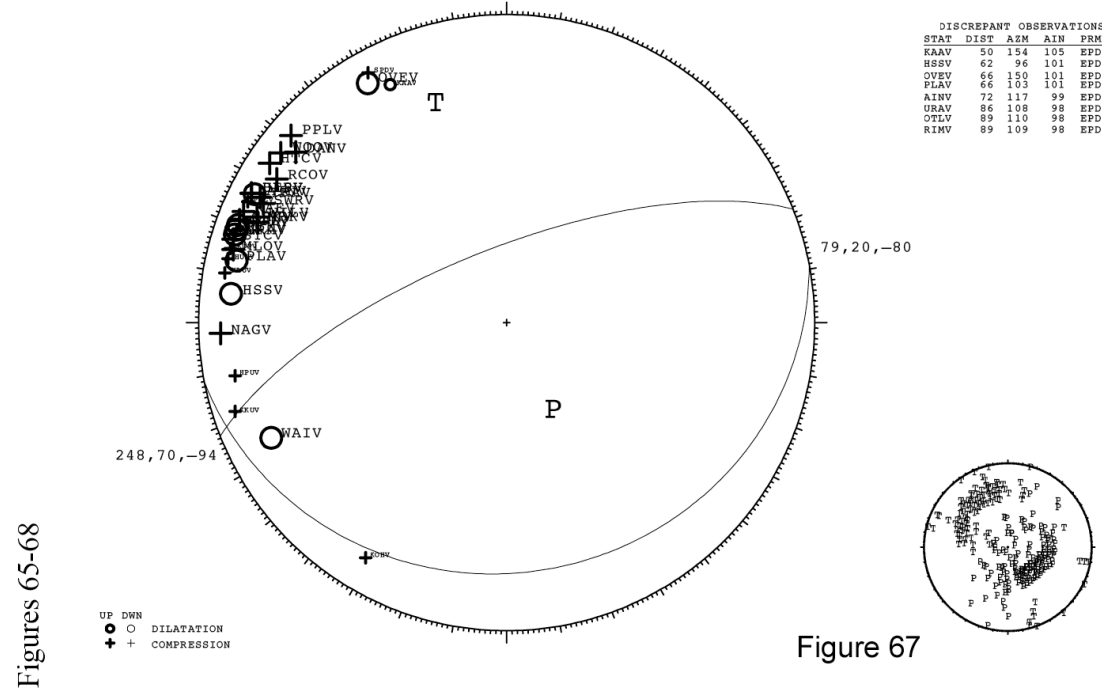

Figure 67

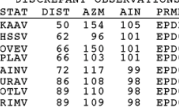

$2008090705: 3448.21$ 1912.69 DEPTH $=36.02 \mathrm{KI}$
$\mathrm{MAG}=3.23 \mathrm{x}$

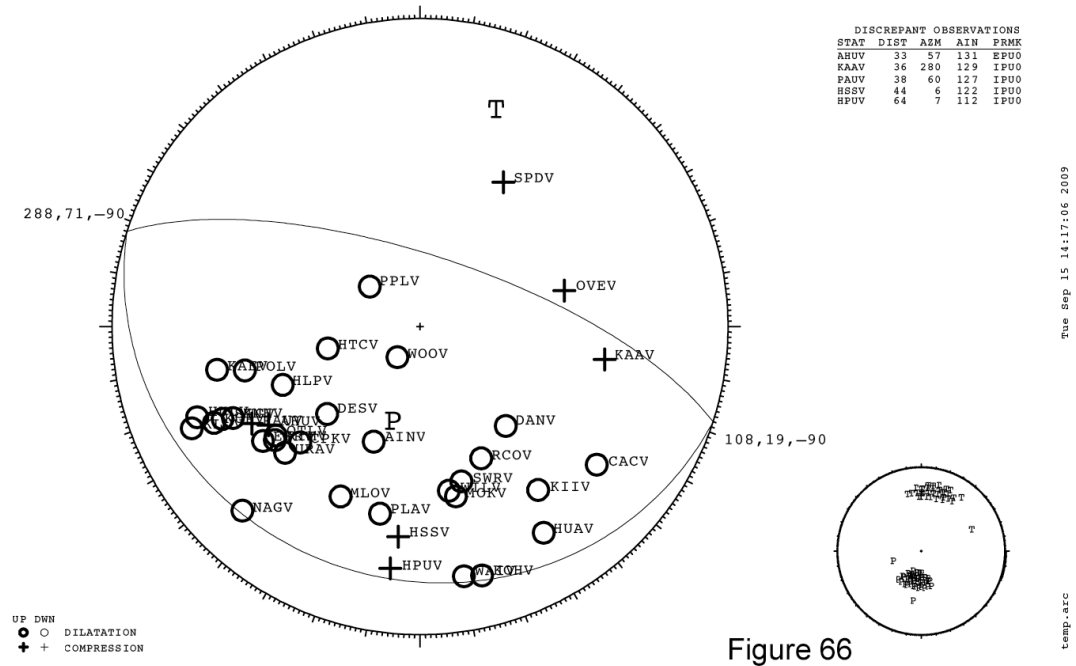

$\begin{array}{ll}\text { RMS }=0.07 \mathrm{~S} & \text { ERH }=0.5 \mathrm{KM} \\ \text { DMIN }=6 \mathrm{KM} & \text { ERZ }=0.9 \mathrm{KM}\end{array}$

E UNCERTAINTY $=2$ $\begin{array}{ll}M I S D R \\ S T D\end{array}=0.68 \quad(+03)$ RARE UNCERTAINTY $=10$

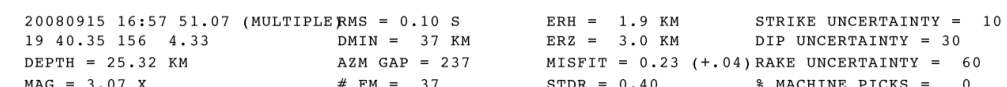

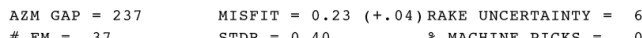

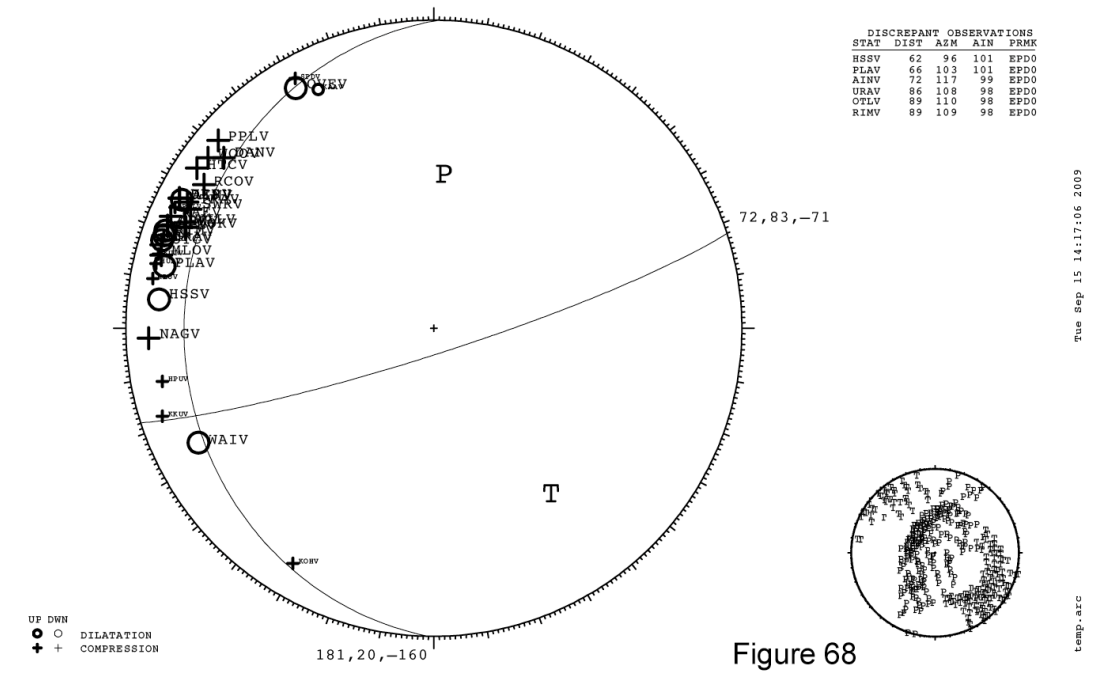

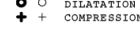



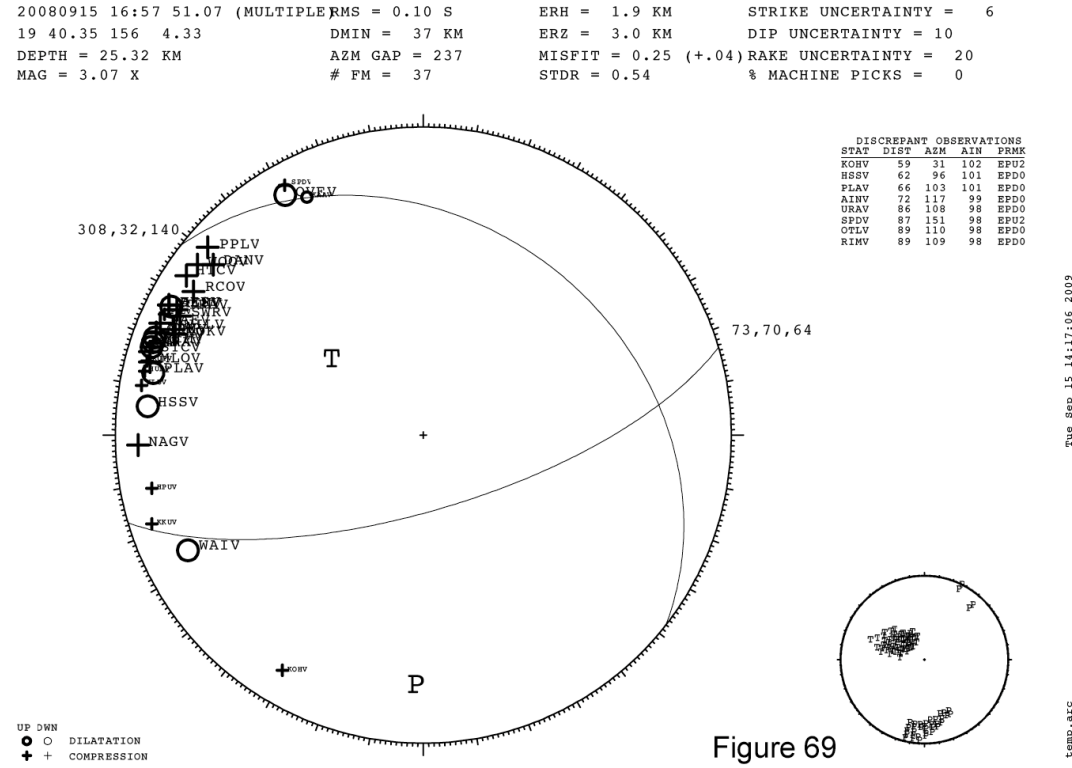

Figure 69

$2008091516: 5751.07$ MULTTPLE RMS $=0.10 \mathrm{~s} \quad$ ERH $=1.9 \mathrm{KM} \quad$ STRIKE UNCRRTAINTY $=20$

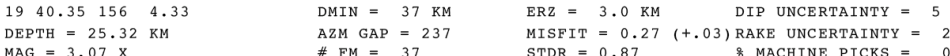
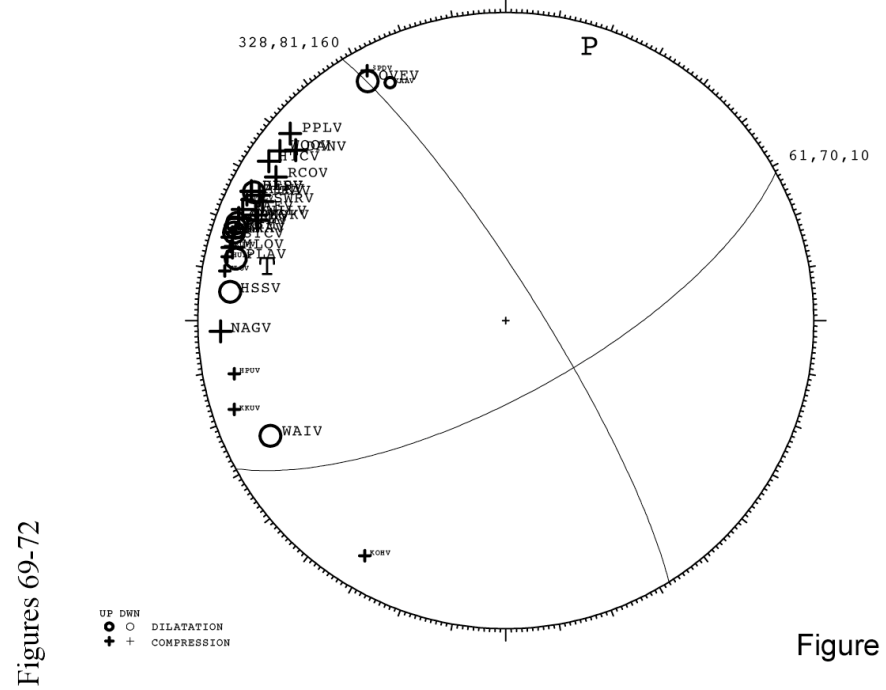

Figure 71

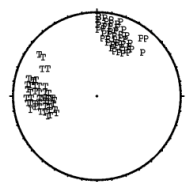

西
$2008091516: 5751.07$ (MULTIPLE RMS $=0.10 \mathrm{~s}$

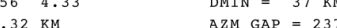
AZM GAP $=23$
$\#$ FM $=37$

$\mathrm{ERH}=1.9 \mathrm{KM}$

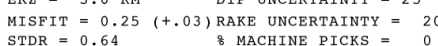

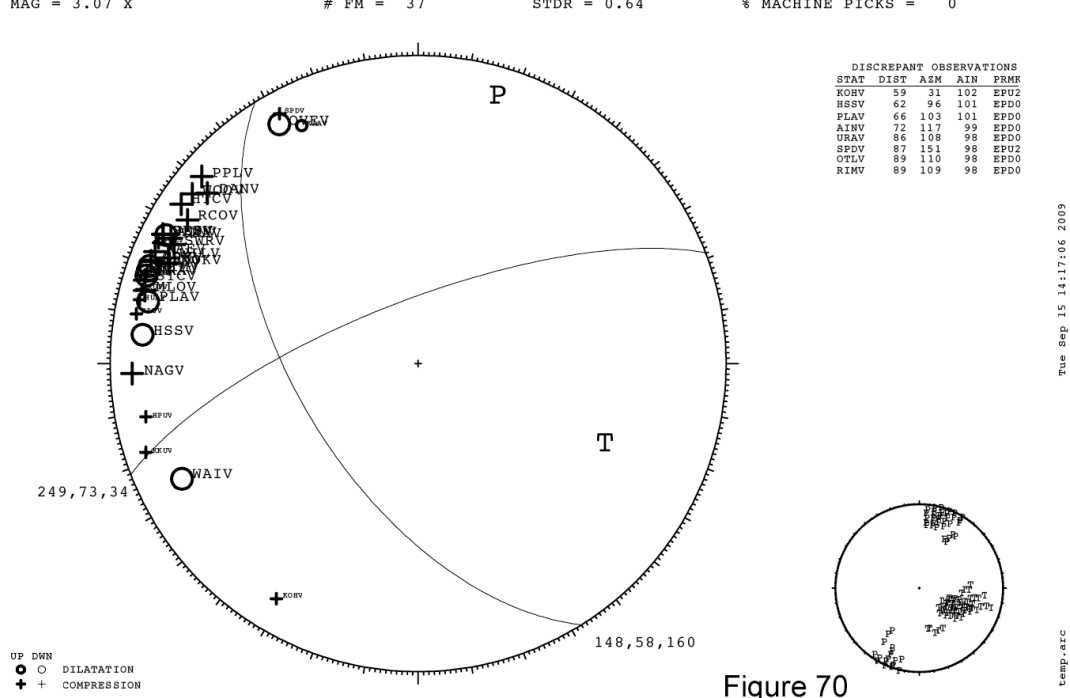

Figure 70

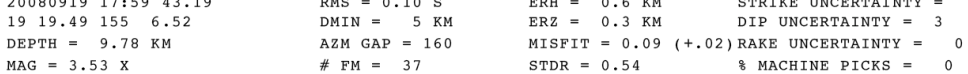

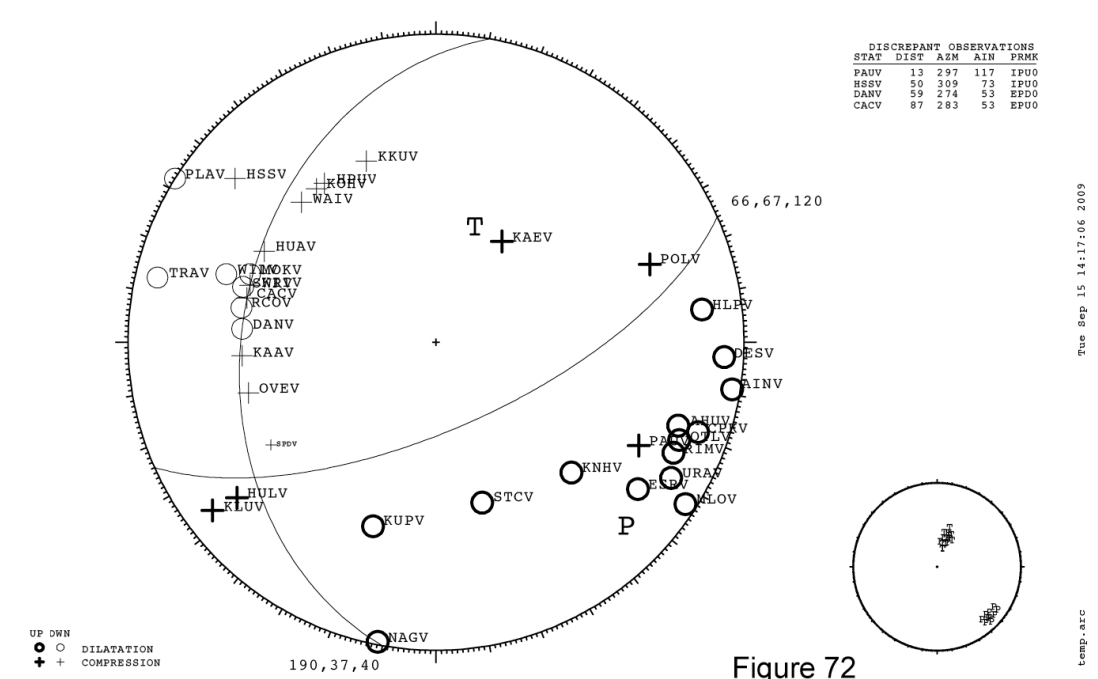

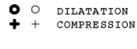

Figure 72 

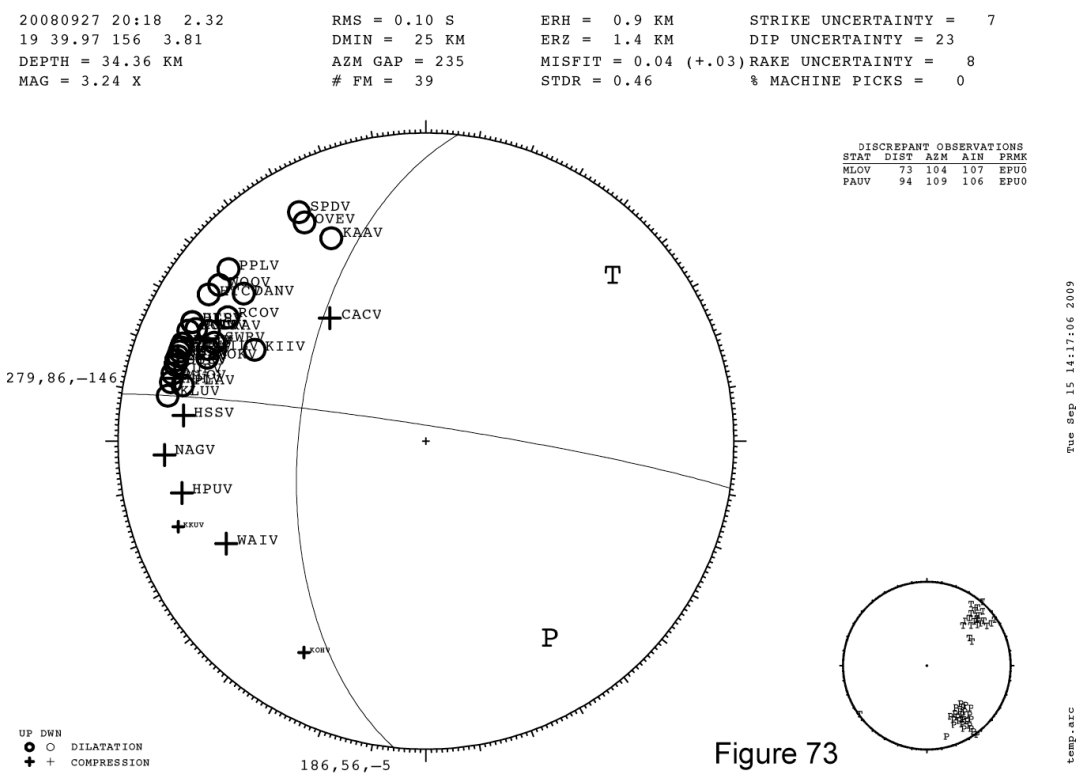

Figure 73

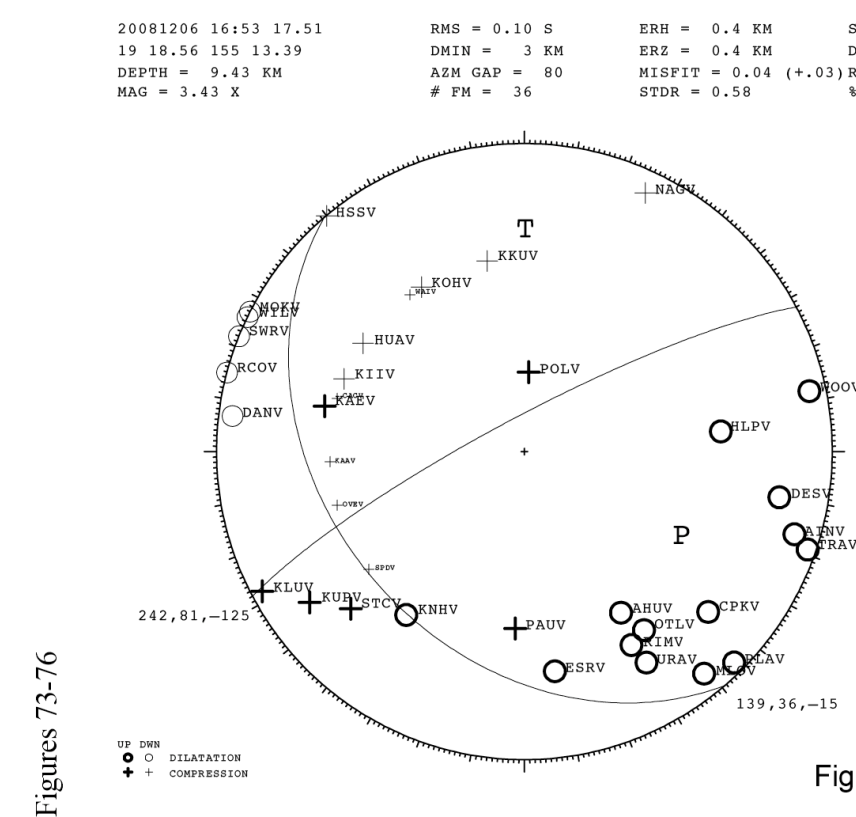

Figure 75 $\begin{array}{llllll}20080927 & 20: 18 & 2.32 & \text { (MULTIPLER RMS }=0.10 \mathrm{~S} & \text { ERH }=0.9 \mathrm{KM} & \text { STRIKE UNCERTATNTY }= \\ 19 & \text { DM.97 } 156 \quad 3.81 & \text { DMIN }=25 \mathrm{KM} & \text { ERZ }=1.4 \mathrm{KM} & \text { DIP UNCERTAINTY }=5\end{array}$

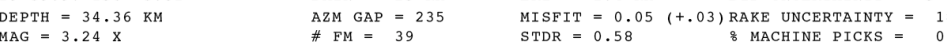

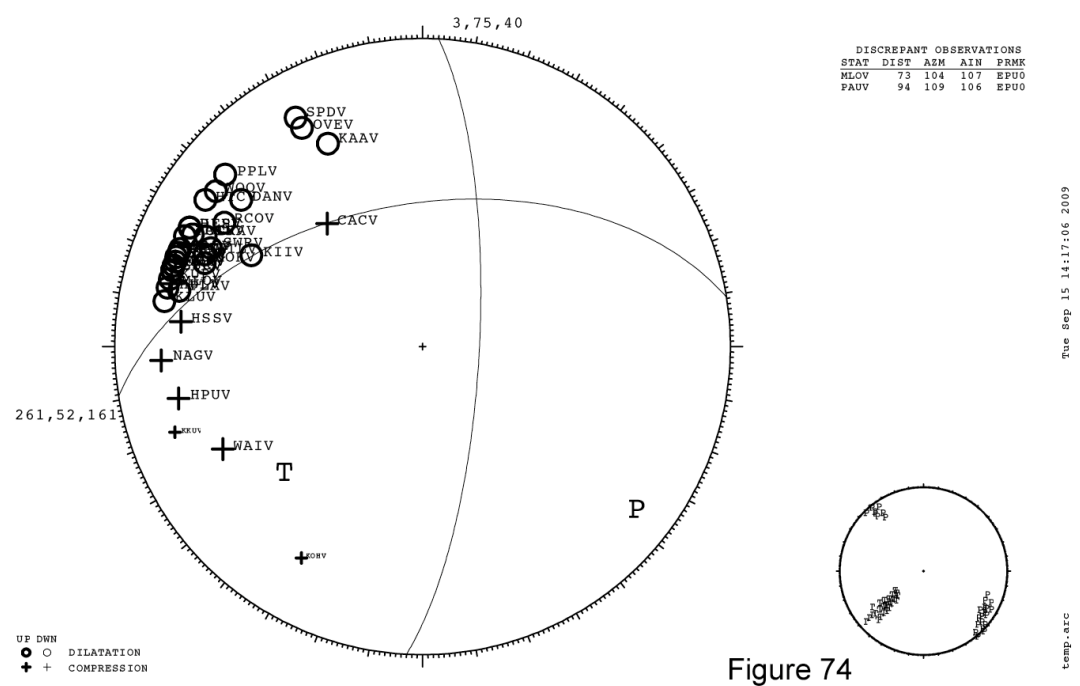

Figure 74

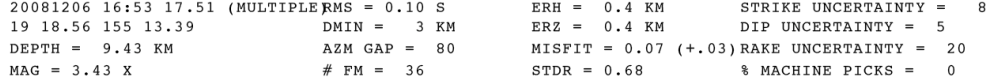

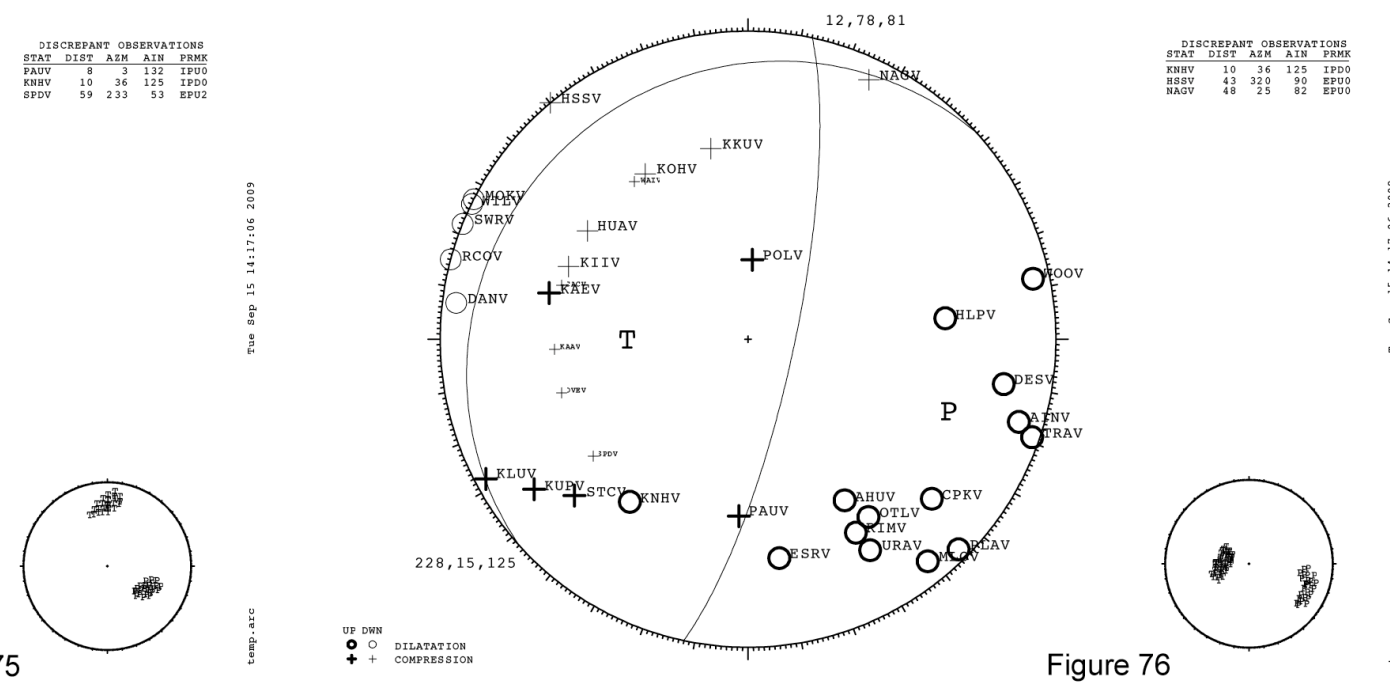



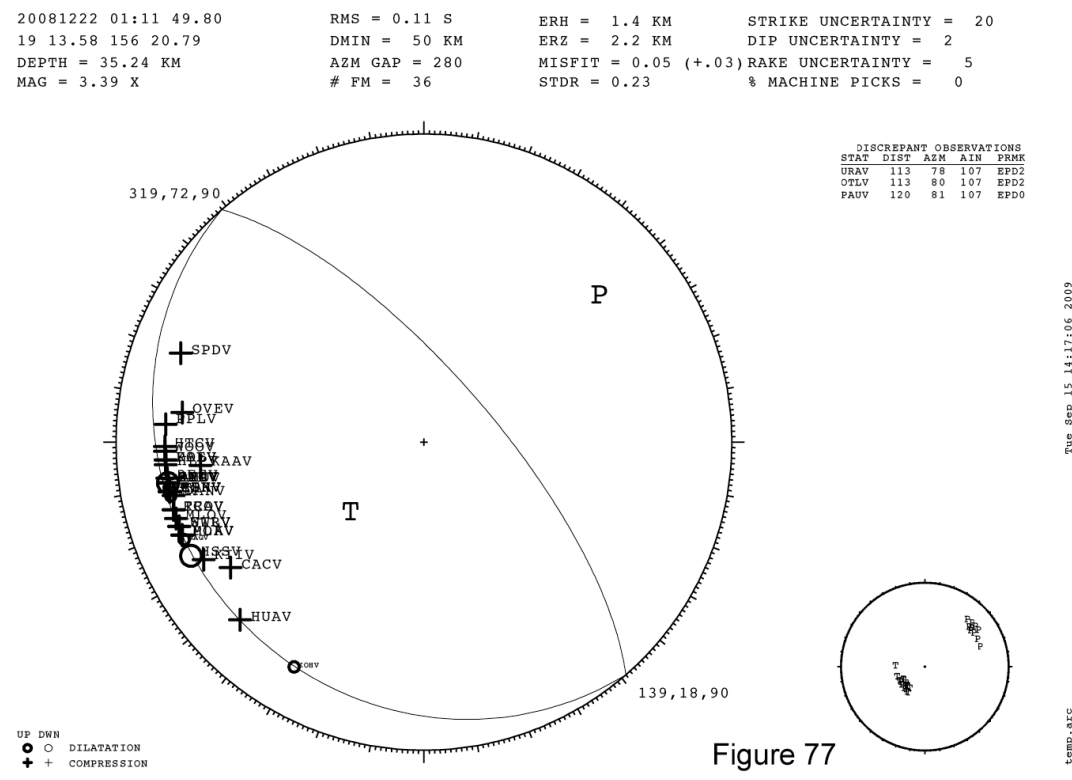

Figure 77

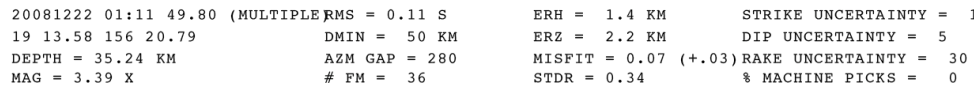

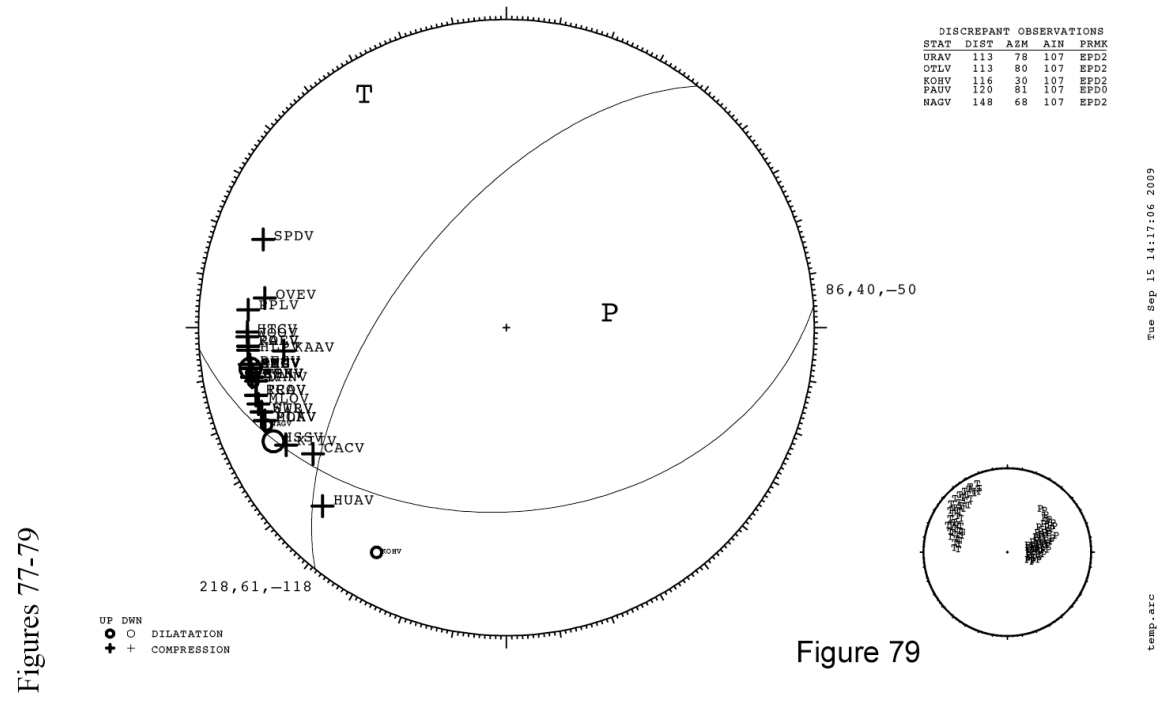

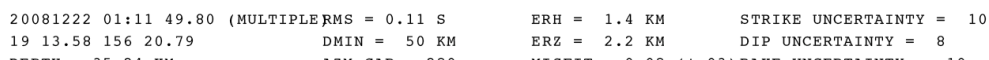

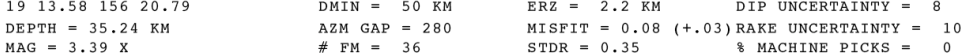

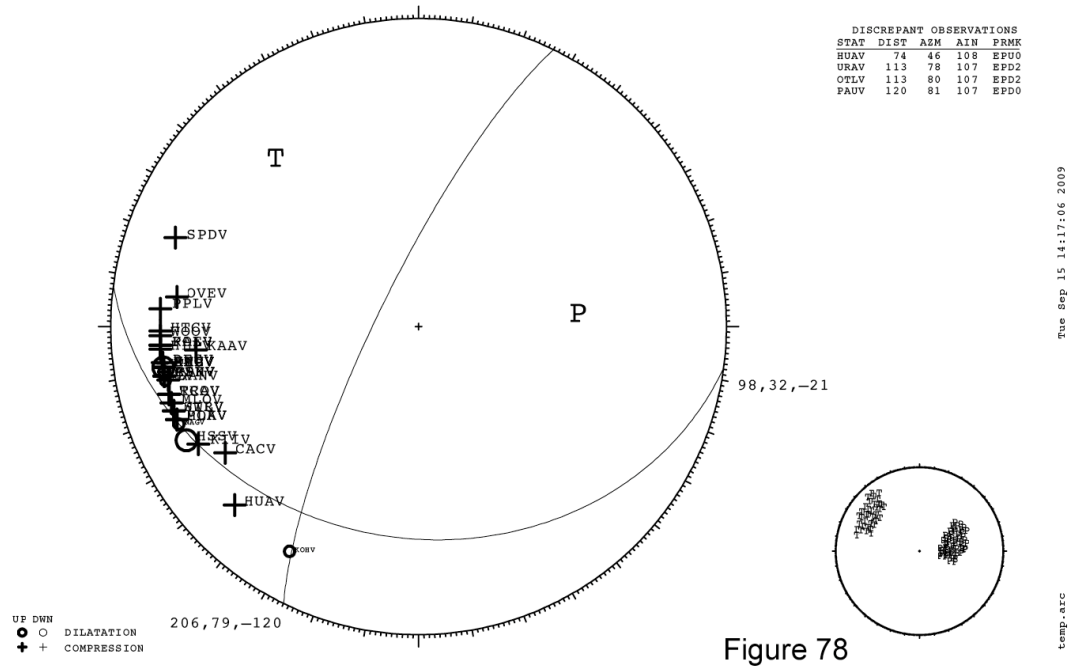



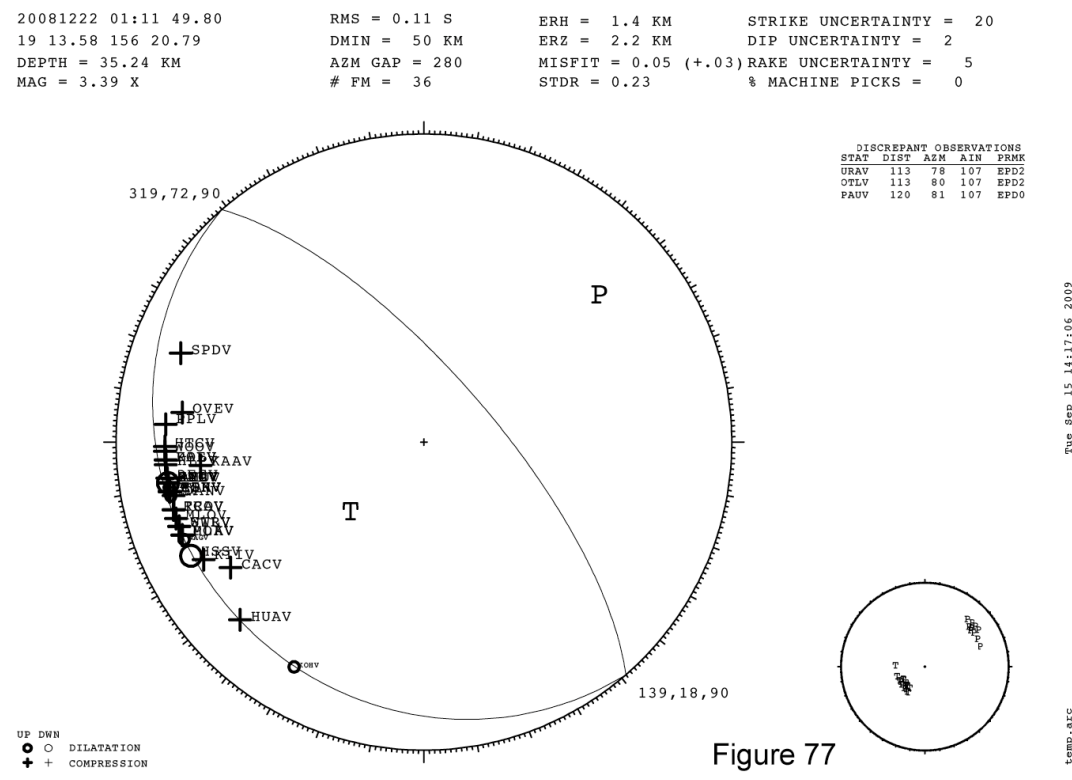

Figure 77

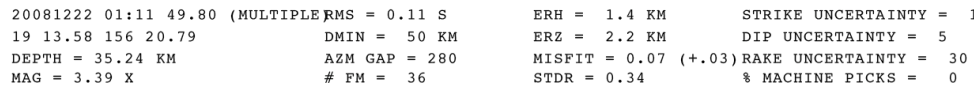

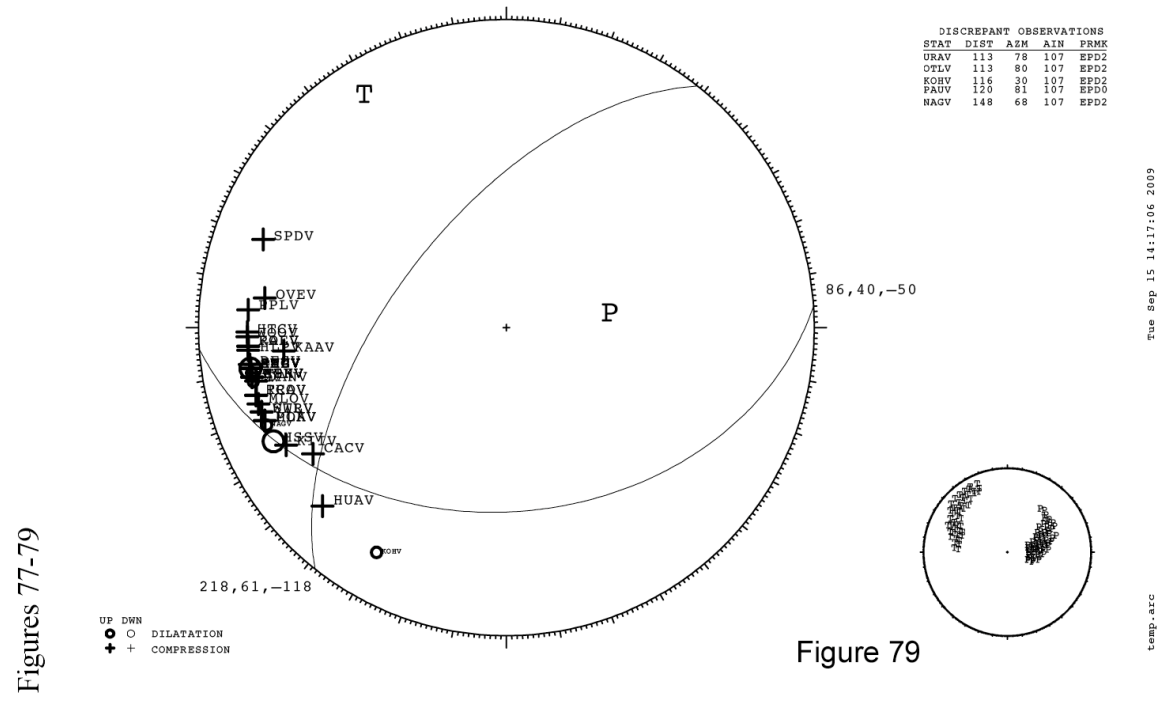

$\begin{array}{llll}2008122201: 1149.80 & \text { (MULTIPLERMS }=0.11 \mathrm{~s} & \text { ERH }=1.4 \mathrm{KM} & \text { STRIKE UNCERTAINTY }=10 \\ 1913.5815620 .79 & \text { DMIN }=50 \mathrm{KM} & \text { ERZ }=2.2 \mathrm{kM} & \text { DIP UNCERTAINTY }=8\end{array}$

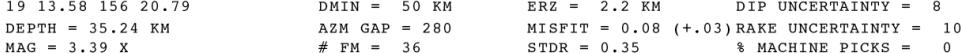

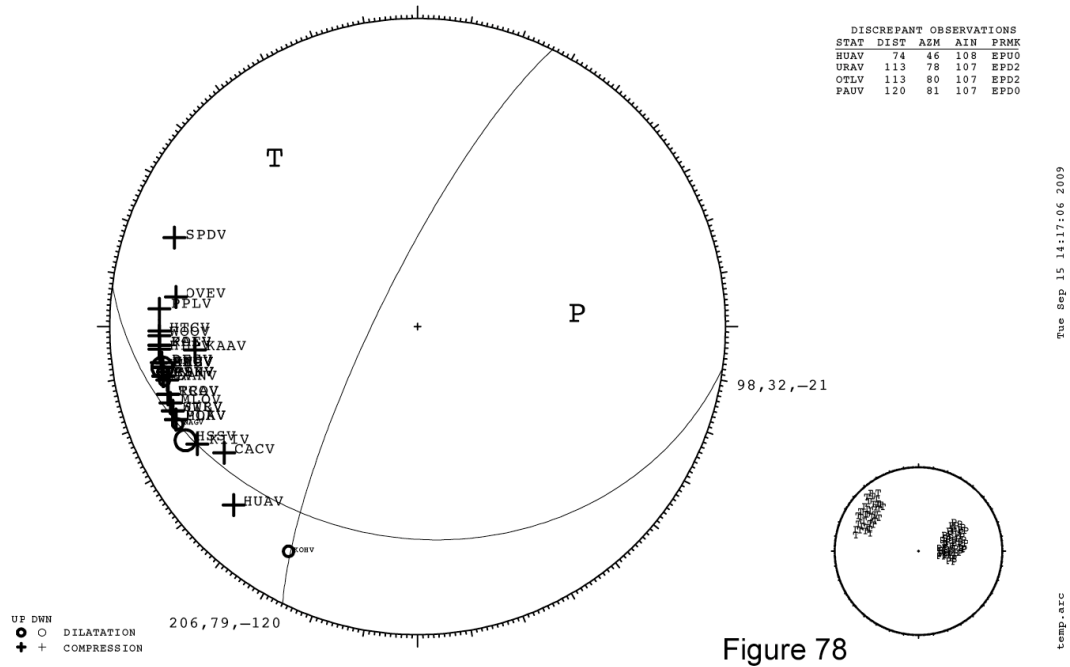

